\author{
Universität Szeged \\ Philosophische Fakultät \\ Germanistische Linguistik
}

\title{
Das es-Gesamtsystem in neuhochdeutschen Nähetexten
}

\author{
Dissertation \\ zur Erlangung des akademischen Grades $\mathrm{PhD}$ in germanistischer Linguistik \\ an der Universität Szeged
}

Vorgelegt von:

Dániel Czicza

Wissenschaftlicher Betreuer:

Prof. Dr. Vilmos Ágel

Szeged 2010 


\section{Vorwort}

Es ist auch bei dieser Dissertion so, dass sie durch Mitwirkung vieler zu Stande gekommen ist. Es gilt meinen Dank an dieser Stelle all denen auszusprechen, die zum Gelingen dieser Arbeit beigetragen haben. Es geht ein sehr großer Dank an Prof. Dr. Vilmos Ágel (Kassel) für die gewissenhafte Betreuung der Arbeit und für seine Geduld. Dr. Rita Brdar-Szabó (Budapest) und Prof. Dr. Gisela Zifonun (Mannheim) waren es, die die erste Fassung dieser Dissertation gelesen und kommentiert haben, wofür ich Ihnen sehr dankbar bin. Dank gebührt auch Prof. Dr. Péter Bassola (Szeged) und Prof. Dr. Mathilde Hennig (Gießen), weil es ohne ihre stete Ermutigung sicherlich nicht möglich gewesen wäre, die Arbeit abzuschließen. Nicht zuletzt halte ich es für wichtig, meiner Familie und meinen Freunden für ihre Unterstützung zu danken.

Kassel, im Herbst 2010

Dániel Czicza 


\section{Inhaltsverzeichnis}

\section{Vorwort}

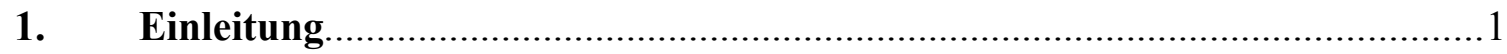

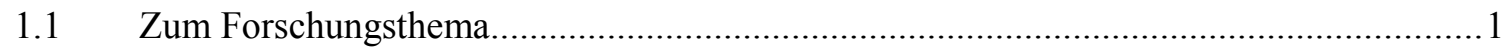

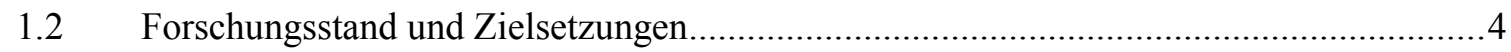

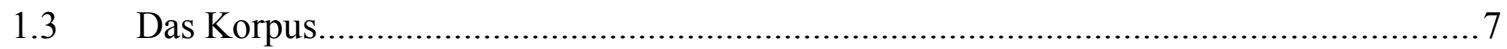

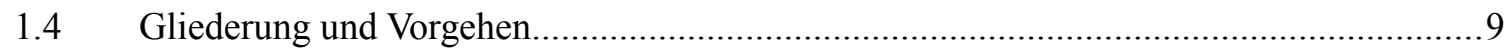

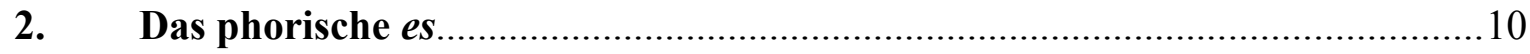

2.1 Verortung der es-Phorik im Kontext der Begriffe

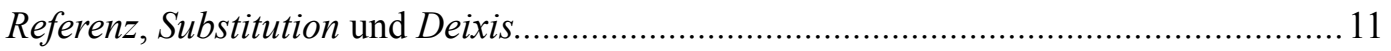

2.2 Semantische und morphosyntaktische Bestimmung der es-Phorik...................................26

2.2.1 Semantische Bestimmung der es-Phorik: implizite Verweise............................................... 31

2.2.2 Semantische Bestimmung der es-Phorik: explizite Verweise...........................................47

2.2.3 Morphosyntaktische Bestimmung der es-Phorik..............................................................58

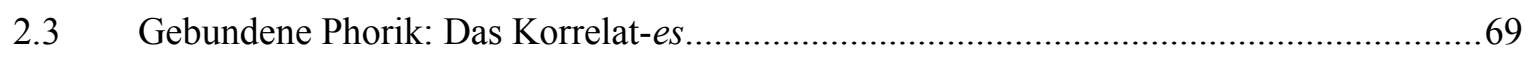

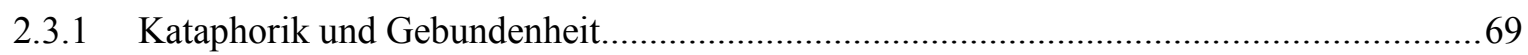

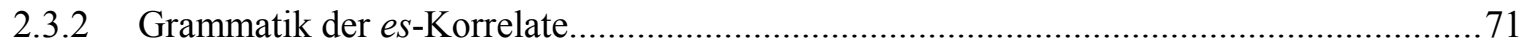

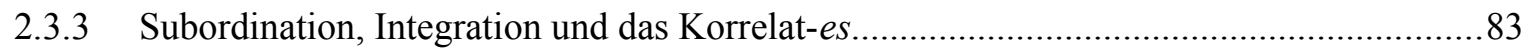

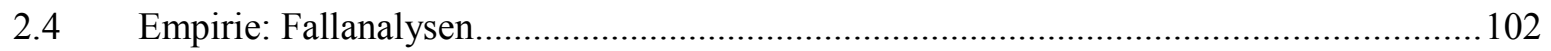

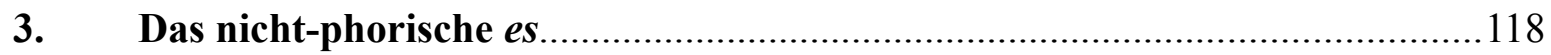

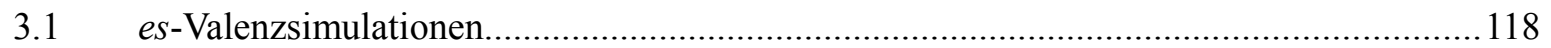

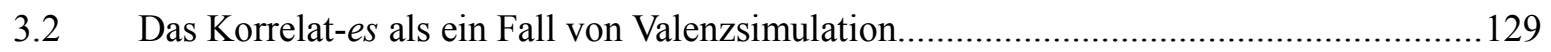

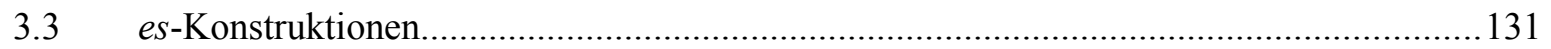

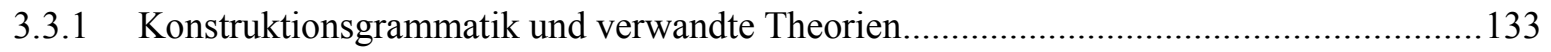

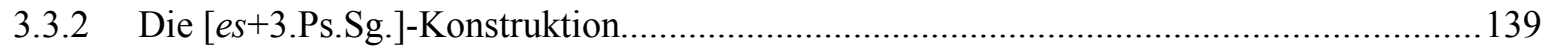

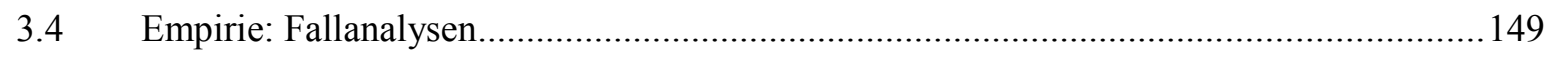

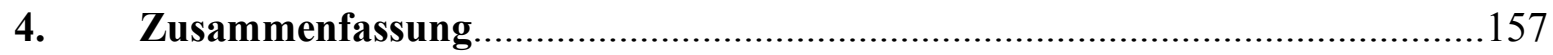

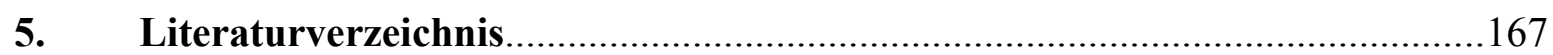

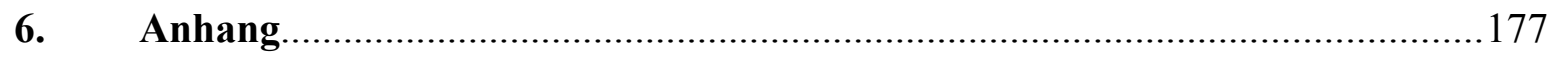

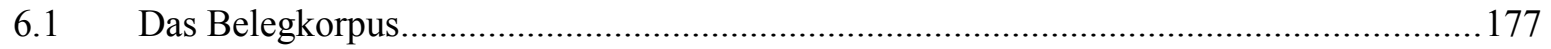

6.2 Die Korpustexte (CD) 


\section{1. $\quad$ Einleitung}

\subsection{Zum Forschungsthema}

Das deutsche Wort es stellt unter linguistischem Aspekt ein vielversprechendes Forschungsthema dar. Es kommt ihm im deutschen Satzbau in formaler wie auch funktionaler Hinsicht eine sehr wichtige Rolle zu, was im Grunde genommen für alle historischen Sprachstufen des Deutschen gilt. Über seine empirische Relevanz hinaus ist das deutsche es immer schon ein Prüfstein grammatischer Theoriebildung in der germanistischen Linguistik gewesen. Es wird in jeder Grammatik des Deutschen mit besonderer Sorgfalt behandelt und es gibt keine bedeutende Grammatiktheorie, die es nicht thematisiert (vgl. etwa Ágel 2000b zur Valenztheorie oder Cardinaletti 1990 zur generativen Grammatik). Herbert Pütz (1975) widmete dem Phänomen sogar eine ganze Monografie. Zu verdanken ist all dies seiner funktionalen Vielfalt und seinem daraus resultierenden grammatischen Verhalten (vgl. Admoni 1976).

Man kann nicht sagen, dass das Thema „es“ unerforscht ist, es gibt zahlreiche Untersuchungen, die die verschiedenen Gebrauchsweisen von es aus grammatischer Sicht behandeln. In der vorliegenden Arbeit wird der Versuch unternommen, das deutsche es sowohl theorie- als auch praxisbezogen teilweise in ein neues Licht zu rücken, indem auf neuere grammatiktheoretische Erkenntnisse zurückgegriffen und dabei ein Korpus herangezogen wird, das sowohl quantitativ wie auch qualitativ neue Möglichkeiten grammatischer Analyse eröffnet. Die Notwendigkeit einer Neuorientierung in der esForschung ergibt sich meiner Meinung nach aus folgenden Gründen:

1. Die bisherige einschlägige Fachliteratur behandelt die es-Typen nicht als ein Gesamtsystem von miteinander zusammenhängenden Funktionen.

2. Seit der Monografie von Pütz (1975) ist keine umfassende Arbeit zu es erschienen, wobei wir in der Grammatikforschung der letzten Jahrzehnte Jahre neue Ideen und Theorieansätze finden können, von deren Anwendung auch im Falle des es neue Erkenntnisse zu erhoffen sind.

3. Den Analysen wurden meistens kleinere Belegsammlungen zu Grunde gelegt oder man arbeitete oft anhand von Beispielen aus der eigenen Kompetenz. 
Es soll nicht bestritten werden, dass die bisherige Forschung wertvolle Ergebnisse bezüglich der Beschreibung des es hervorgebracht hat. Es wurde in zahlreichen, teils mit unterschiedlichem theoretischen Apparat arbeitenden Analysen bestätigt, dass das deutsche es über zahlreiche Funktionen verfügt, was grammatische Konsequenzen nach sich zieht. Selbstverständlich war diese Erkenntnis gar nicht neu - wir finden sie schon in älteren Grammatiken -, aber der intensiven Beschäftigung mit dem breiten funktionalen Spektrum von es zufolge sind etliche Klassifikationen zu Stande gekommen (vgl. z.B. Pütz 1975) und man findet auch eine Menge von Fallstudien zu einzelnen Verwendungsweisen von es (vgl. z.B. Zifonun 1995 zum nicht-phorischen es). Vernachlässigt wurden dabei oft die zwischen den einzelnen Gebrauchsweisen bestehenden Zusammenhänge. Der Grund dafür besteht darin, dass die Mehrheit der Arbeiten synchron orientiert ist. Diese Herangehensweise hat zur Folge, dass die verschiedenen es-Typen als solche erscheinen, die zueinander in keinem Verhältnis stehen. Problematisch ist dieses Endergebnis vor allem, weil es aus sprachhistorischer Sicht nicht haltbar ist (vgl. die Darstellungen etwa bei Behaghel 1923, 1928 oder Ágel 2003: 25 zu es scheint). Eine mögliche Lösung des geschilderten Problems wäre m.E., dass man von einem es-Gesamtsystem ausgeht, in dem die verschiedenen esFunktionen nicht separat erscheinen. Darauf hat Admoni bereits 1978 aufmerksam gemacht und die Überlegungen in der vorliegenden Arbeit stützen sich vielfach auf diesen Systemgedanken.

Zur Modellierung eines es-Gesamtsystems bieten sich mehrere Forschungsideen und Theorieansätze der letzten Jahrzehnte an, die neue Einblicke ins alte Thema ermöglichen könnten:

- Für eine auch sprachgeschichtlich fundierte Herangehensweise können Grammatikalisierungstheorien einen guten Hintergrund bedeuten. Zieht man z.B. so genannte phorische es-Vorkommen, die auf Vorerwähntes oder Nachfolgendes Bezug nehmen, in Betracht, so lässt sich dort ein semantisch-grammatischer Wandel nachvollziehen. Der Abbau semantisch-grammatischer Restriktionen zwischen es und dem Bezugsglied, wie es beim Referenzwandel von es der Fall ist, ist ein typischer Zug von Grammatikalisierungsprozessen. Aber auch das nicht-phorische es in festen Konstruktionen (wie z.B. in es regnet) erlaubt eine Annäherung, die auf Grammatikalisierung basiert. Dadurch, dass man die verschiedenen Phasen der 
Entwicklung von es mit Hilfe von Grammatikalisierungsprozessen beschreibt, wird auch eine kontinuative Modellierung des ganzen Systems möglich.

- Das Vorkommen des es in festen Verbindungen gibt den Anlass dazu, bei der Analyse in erster Linie nicht-phorischer, aber teils auch phorischer esGebrauchsweisen Grammatikmodelle heranzuziehen, die sich das Ziel setzen, das Formelhafte in der Sprache $\mathrm{zu}$ erklären und $\mathrm{zu}$ analysieren. Die Idee, polylexikalischen, nicht strikt kompositionellen Einheiten auch in der Grammatik eine größere Rolle zukommen zu lassen, bildet den Ausgangspunkt auch in den verschiedenen Ansätzen der so genannten Konstruktionsgrammatik (Construction Grammar). Diese Grammatiktheorie hat seit den Arbeiten von Charles Fillmore (s. etwa 1989) und in der germanistischen Linguistik vor allem seit dem Erscheinen der Arbeit von Adele Goldberg (1995) einen stürmischen Aufschwung erlebt.

- Es gibt vielversprechende Forschungsansätze, welche die verschiedenen Perspektivierungsmöglichkeiten der Grammatik zum Gegenstand machen (vgl. z.B. Ickler 1990). Im Falle des es in impersonalen Konstruktionen (z.B. es schneit/donnert) handelt es sich beispielsweise um Ereignisse, die durch die feste Verbindung von es und der Verbform (3. Person Singular) in besonderer Weise kodiert werden. So ist es möglich, Handlungsverben wie z.B. klopfen durch eine solche Konstruktion (es klopft) neu (nämlich als Geschehen) zu perspektivieren. Unter Einbeziehung dieser Forschungsidee eröffnen sich neue Perspektiven auch für eine valenztheoretische Analyse von es (vgl. Ágel 2000b und Ickler 1990).

Die hier kurz dargestellten neueren Erkenntnisse in der Grammatiktheorie ermöglichen m.E. ein teilweise neues Herangehen an das zu behandelnde Phänomen. Auf Grund der obigen Überlegungen lässt sich sagen: Eine moderne Behandlung des Themas soll m.E.:

1. gesamtsystematisch ausgerichtet sein,

2. neuere Erkenntnisse der Grammatikforschung mit berücksichtigen und

3. sich auf ein breites Textmaterial stützen, um empirische Validität zu sichern. 


\subsection{Forschungsstand und Zielsetzungen}

Die Forschung der letzten Jahrzehnte konzentrierte sich in erster Linie auf zwei Gesichtspunkte. Einerseits hat man versucht, die veschiedenen es-Typen auf Grund syntaktischer (Pütz 1975, Helbig/Buscha 1984) oder syntaktisch-referentieller (Askedal 1990, Admoni 1976) Kriterien zu klassifizieren, um der Vielfalt auch theoretisch Rechnung zu tragen. Eine wichtige Motivation dafür war vielerorts die DaF-Perspektive, weil der korrekte Gebrauch von es vielen Studierenden nicht-deutscher Muttersprache große Schwierigkeiten bereitet(e). Ein ,einfacherer“ Grund für Klassifizierungen könnte auch die Absicht (gewesen) sein, Ordnung zu schaffen, um nachvollziehen zu können, dass es sich bei den verschiedenen es-Sätzen tatsächlich nicht um dasselbe es-Element handelt. Neben den Klassifikationsversuchen gibt es etliche Einzeluntersuchungen, die entweder das Korrelat-es (vgl. z.B. Latour 1981, Marx-Moyse 1983, Ulvestad/Bergenholtz 1979, 1983; Sandberg 1998, Zitterbart 2002) oder das expletive es (vgl. z.B. Lenerz 1985, Cardinaletti 1990, Hoening 1994, Zifonun 1995, Szatmári 1998, Scheibl 2000) thematisieren. Beim Korrelat-es geht es darum, Kriterien für Obligatheit oder Fakultativität von es im Matrixsatz $\mathrm{zu}$ finden, was wiederum auch aus didaktischer Sicht von großer Bedeutung ist. Was das expletive es anbelangt, so wurde und wird über seinen syntaktischen Status und das Vorhandensein bzw. Nicht-Vorhandensein eines semantischen Gehalts diskutiert.

Nun ist es aber auch möglich, nicht nur auf Unterschiede aufmerksam zu machen und diese dadurch zu rechtfertigen, dass man separate es-Klassen aufstellt, deren Elementen klassenspezifische Merkmale jeglicher Art zugeschrieben werden. Es könnten und sollten auch Gemeinsamkeiten zwischen den verschiedenen es-Typen betont und untersucht werden. Die Versuche, die sich um die Aufstellung von getrennten Klassen bemühen, können in der jeweiligen Syntaxtheorie wichtig sein, indem sie die Beschreibungsadäquatheit der Theorie zu untermauern versuchen. Aus didaktischer Sicht ist die Behandlung von es in separaten Gruppen begründbar, vor allem was Stellung und Setzung von es angeht. Doch man dürfte über die Tatsache nicht hinwegsehen, dass die voneinander unabhängig dargestellten es-Typen doch etwas miteinander zu tun haben. Die Gefahr in einem „Schaffen wir Ordnung!“-Prinzip liegt darin, dass man zu denken beginnt, die verschiedenen es-Funktionen hätten keinerlei Beziehung zueinander, was auch den sprachgeschichtlichen Fakten widerspricht (vgl. hierzu Behaghel 1923: 316ff.). So kritisiert Admoni (1976: 225) die Monografie von Pütz (1975) - die bis heute ausführlichste Arbeit 
über es - wie folgt: „Das Buch bleibt in operationell gewonnenen Teilergebnissen stecken, ohne jeglichen Versuch, die Vielfalt der GW [Gebrauchsweisen, D.C.] von es als ein zusammenhängendes System zu betrachten $[\ldots]^{\text {“‘. }}$

Die vorliegende Arbeit setzt sich das Ziel zu zeigen, dass sich die verschiedenen esGebrauchsweisen auch als ein zusammenhängendes System beschreiben und modellieren lassen. Ich werde versuchen, Zusammenhänge anhand von Beispielen darzustellen und auf Übergänge zwischen verschiedenen Vorkommensweisen von es hinzuweisen. Es wird von zwei Grundtypen ausgegangen, von einem phorischen und einem nicht-phorischen. Diese Grobdifferenzierung wird funktional gerechtfertigt: Der phorische Typus ist textbezogen beschreibbar, erfüllt also wichtige Funktionen im Text (Verweis auf vorerwähnte Einheiten unterschiedlichen Umfangs und verschiedener Art). Das nicht-phorische es ist grundsätzlich ein formales Element mit (auch semantisch motivierter) syntaktischer Funktion. In beiden Fällen ist mit Subtypen zu rechnen, die sich stufenweise beschreiben lassen, wodurch sogar eine Verbindung der zwei Grundtypen möglich wird.

Aus den oben angedeuteten Desiderata in der einschlägigen Forschung, dem Rückgriff auf neuere grammatiktheoretische Erkenntnisse und den Möglichkeiten empirischer Analyse durch das zu Grunde gelegte Korpus ergeben sich für die vorliegende Arbeit folgende Zielsetzungen:

1. phorisches es

$1 a$ Es sollen Arten und Typen der es-Phorik unter semantischem und morphsyntaktischem Gesichtspunkt erörtert werden. Das Belegmaterial ermöglicht es, bisher nicht oder nur am Rande behandelte phorische es-Vorkommen detaillierter $\mathrm{zu}$ beschreiben. Hierbei geht es vor allem um eine Unterscheidung expliziter von impliziter Phorik sowie um die Darstellung nicht-korrespondierender Bezüge auf Nominalphrasen durch es .

$1 b$ Das Belegmaterial und der Begriff der Integration ermöglichen es, das Korrelat-es unter einem anderen Gesichtspunkt $\mathrm{zu}$ untersuchen als es in der bisherigen Forschungsliteratur geschehen ist. 
2. nicht-phorisches es

$2 a$ Es soll der Begriff der Valenzsimulation auf nicht-phorische es-Verwendungsweisen angewendet werden.

$2 b$ Es soll der Versuch unternommen werden, bestimmte Elemente der Construction Grammar (im Folgenden Konstruktionsgrammatik) auf das nicht-phorische es zu beziehen.

Im Hintergrund dieser Zielsetzungen soll die von Wladimir Admoni (1976: 222) stammende Idee stehen, die verschiedenen es-Vorkommen als ein Gesamtsystem zu betrachten:

[...] wäre überhaupt nicht ihre lineare Aufzählung [die Aufzählung der Gebrauchsweisen von es, D.C.] tauglich, sondern ihre Fixierung in der Form eines Vielecks, in dem die Ecken $(=\mathrm{GW})$ miteinander sowohl durch Kanten als auch durch mehrere Sekanten verbunden sind, die die gemeinsamen Merkmale der GW bezeichnen.

Dieser Gedanke ist nicht neu, fand und findet in der Forschung jedoch nur ansatzweise Beachtung. So taucht er bei Szatmári (1998), Ágel (2000b) und Zitterbart (2002a, b) auf. Zu erwähnen ist außerdem Askedal (1990). In seinem Artikel über die syntaktischreferentiellen Funktionen des es gibt es Hinweise auf Zusammenhänge zwischen verschiedenen es-Arten (vgl. dort Typ, 3, 5 und 9). Die sprachgeschichtlichen Ausführungen in Lenerz (1985) sind ebenfalls bemerkenswert. Sie gehen der Frage nach, wie sich das expletive es in den verschiedenen Sprachstufen des Deutschen und anderer germanischer Sprachen entwickelt hat. Lenerz verfolgt aber nicht das Ziel, ein integrierendes Modell zu entwerfen, sondern er versucht den Sprachwandel, „der sich im zunehmenden Auftreten des expletiven es in nicht-erster Position niederschlägt [...] als Wandel der strikten Subkategorisierung der betreffenden lexikalischen Elemente“ zu erfassen (1985: 118). 


\subsection{Das Korpus}

Meine Untersuchungen konzentrieren sich auf den Zeitraum 1650-2000. Die Grundlage der theoretischen und empirischen Analysen bildet ein Korpus, das im Rahmen des von Vilmos Ágel (Universität Kassel) geleiteten Projekts „Sprachstufengrammatik 1650-2000“ zusammengestellt wurde. Da sich die bisherigen Forschungen meistens entweder auf kleinere Belegsammlungen oder selbst kreierte Beispiele stützten, erschien es mir sinnvoll, meiner Arbeit ein größeres Textmaterial zu Grunde zu legen, wodurch die Theorie auch empirisch abgesichert werden kann. Das im genannten Projekt verwendete Korpus ermöglicht eine solche empirische Absicherung. Es eröffnet unter sowohl quantitativem wie auch qualitativem Aspekt neue Perspektiven. Einerseits sind Menge und Breite des zu bearbeitenden Materials größer als in früheren Arbeiten, es werden Texte aus mehreren Jahrhunderten herangezogen. Andererseits handelt es sich bei diesem Korpus um Texte, die das so genannte Nähesprechen (Koch/Oesterreicher 1985) repräsentieren. Wie oben bei den Zielsetzungen bereits erwähnt, werden dadurch Analysen von Belegen möglich, die in anderen Texten nicht in dieser Menge zur Verfügung stehen.

Das Korpus beinhaltet insgesamt 13 Texte, die hinsichtlich des Zeitraums 1650-2000 auf sieben Abschnitte verteilt sind:

\section{Abschnitt I (1650-1700)}

Güntzer $(=\mathrm{G})$

Bauernleben (= BL)

\section{Abschnitt II (1701-1750)}

Nehrlich (= NE)

Meister Dietz (= MD)

\section{Abschnitt III (1751-1800)}

$\operatorname{Bräker~(=~B)~}$

Jägerbursch (=J) 
Dienstmagd (= DM)

Laible (= L)

\title{
Abschnitt V (1851-1900)
}

Koralek (= K)

Schiele $(=\mathrm{SCH})$

\author{
Abschnitt VI (1901-1950) \\ Liebesbriefe (= LB) \\ Schwabengängerin (= SCHG)
}

\section{Abschnitt VII (1951-2000) \\ Sprachbiographien $(=\mathrm{SB})$}

Alle Texte liegen in digitaler Form vor und haben einen Umfang von ca. 12.000 Wörtern. Der Bezug auf die Belegstellen richtet sich immer nach den Seitenangaben, die in den digitalen Dokumenten angegeben sind. Bei dem Text Güntzer handelt es sich dabei um die in der Druckversion vorhandenen Seitenangaben, bei Jägerbursch sind es die Abschnittsziffern, die von der transkribierenden Person eingetragen wurden. Im Falle von Liebesbriefe beziehen sich die Zahlen auf die jeweilige Textzeile. Der Verweis auf die entsprechende Belegstelle findet sich immer in Klammern nach dem jeweiligen Beispiel. Dabei wird das oben angegebene Kürzel verwendet, das ganz am Ende des jeweiligen Belegs steht. Da es Belege gibt, in denen eventuell mehrere es-Vorkommen auftauchen, wurde immer das kursiv gesetzt, zu dem in der jeweiligen Analyse Aussagen gemacht werden.

Es wurde darauf geachtet, dass das Material in Bezug auf Textsorten möglichst einheitlich gehalten wird. So stellen die Korpustexte Lebensbeschreibungen und Briefe dar. 


\subsection{Gliederung und Vorgehen}

Die Arbeit gliedert sich in zwei thematische Hauptkapitel (Kapitel 2 und 3) und wird durch eine Zusammenfassung (Kapitel 4) abgerundet, in der die wichtigsten Ergebnisse festgehalten und relevante Konnexionen zwischen den einzelnen es-Typen dargelegt werden. Den Überlegungen in den beiden Hauptkapiteln liegen jeweils entsprechende theoretische Bausteine zugrunde. So werden in Kapitel 2 u.a. Begriffe wie Phorik, Referenz, Deixis und Korrelat herangezogen. Im Mittelpunkt stehen dabei die Annahme impliziter vs. expliziter Phorik, die semantische sowie morphosyntaktische Beschreibung textueller Verweise durch es und das Korrelat-es (Kapitel 2.2 und 2.3). In dem hauptsächlich grammatiktheoretisch ausgerichteten Kapitel 3 kommt hingegen Theoremen aus der Valenztheorie und der Konstruktionsgrammatik eine zentrale Rolle zu. Dabei sollen bisherige Erkenntnisse mit Rücksichtnahme auf neuere Ergebnisse der Grammatikforschung kritisch überprüft und ergänzt werden. So wird zunächst auf den Begriff der Valenzsimulation zurückgegriffen (3.1). Diesem Abschnitt folgen eine Analyse des Korrelat-es unter valenztheoretischem Aspekt (3.2) bzw. Überlegungen zur Beschreibung nicht-phorischer es-Vorkommen im Rahmen der Konstruktionsgrammatik (3.3). Die Festlegungen in den theoretischen Komponenten sollen in beiden Hauptkapiteln durch empirische Fallanalysen (Kapitel 2.4 und 3.4) präzisiert und erweitert werden. Diese Art von Strukturierung erklärt sich durch die Arbeitsteilung zwischen Theorie und Empirie: Der theoretische Teil beinhaltet grundlegende Überlegungen zur Typologie, Generalisierungen und wichtige Festlegungen. In den empirischen Kapiteln 2.4 und 3.4 sollen durch die Analysen Feindifferenzierungen vorgenommen und für die Grundtypologie interessante Belege beschrieben werden. Dies wiederum soll die Rückkopplung der Empirie an die Theorie gewährleisten und der Herstellung eines Konnexes zwischen diesen beiden dienen. 


\section{Das phorische es}

In der Fachliteratur wird einhellig zwischen phorischem und nicht-phorischem es unterschieden. Dabei ist diese Einhelligkeit eher eine konzeptionelle, denn hinsichtlich der Begrifflichkeit besteht kein Konsens. M.a.W.: Es wird grundsätzlich erkannt, dass es Gebrauchsweisen von es gibt, die vor dem Hintergrund ihres Funktionierens in Texten zu interpretieren, d.h. textkonstituierend sind (i) (Harweg 1979: 18), während andere esGebrauchsweisen über keine textkonstituierende Kraft verfügen (ii):

(i) Ich halte es also für unwahrscheinlich, daß dieses ständig geübte Vokabular nicht auch in der Familie auftauchte. Das geht wahrscheinlich gar nicht anders. ... Und es tauchte auch auf. (17) (SB)

(ii) Welche Unterschiede gab es zwischen der östlichen und der westlichen Mediensprache? (8) (SB)

Sowohl (i) als auch (ii) umfassen jeweils einen wesentlich größeren Satz an möglichen Daten, als hier gezeigt, wobei Differenzierungen verschiedener Art innerhalb beider Gruppen vorgenommen werden können und auch müssen. Für die Diskussion hier reicht es aber, zwei „Paradebeispiele“ anzuführen, die als Orientierung dienen sollen. Die Basis der Überlegungen in Kapitel 2 bildet (i). Grundsätzlich gilt, dass theoretische Festlegungen anhand weniger Beispiele vorgenommen werden, die nur die wichtigsten Züge der esPhorik zeigen sollen. Überlegungen zu spezifischen, diskussionswürdigen Belegen finden sich in den empirischen Kapiteln (2.4 und 3.4).

Während zur Terminologie beim nicht-phorischen es (ii) dank der Diskussion um seinen grammatiktheoretischen Status eine Menge an einschlägigen Darstellungen vorliegt, so lässt sich im Falle des phorischen es (i) konstatieren, dass terminologischen und damit im Zusammenhang theoretischen Fragen kaum nachgegangen wird. Auch die verschiedenen Positionen bei der Beurteilung des Korrelat-es beschränken sich im Großen und Ganzen auf dessen Obligatorik/Fakultativität und auf Faktoren, die Setzung und Nicht-Setzung des Korrelates steuern. Dabei begegnet man in der einschlägigen Fachliteratur Grundbegriffen wie Referenz (von Polenz 1985: 129; Askedal 1990: 213), Phorik (IDS-Grammatik 1997: 
38), Substitution (Harweg 1979: 20) ${ }^{1}$ und Wiederbezug (von Polenz 1985: 137), die auf das phorische es allerdings teilweise unreflektiert bezogen werden, vgl. etwa die Gleichsetzung von Referentialität und Phorik (Hinweisfunktion) in Askedal (1990: 213). Wie weiter unten $\mathrm{zu}$ zeigen sein wird, ist die saubere Trennung der verwendeten Begriffe möglich und notwendig. Ausgehend von diesem Standpunkt sollen im Folgenden folgende Fragen diskutiert werden:

a) Was ist Phorik und in welchem Begriffskontext ist sie angelegt? (2.1)

b) Wie sehen von es-Phorik erfasste Objekte aus? (2.2) Dabei geht es im Wesentlichen um

ba) die semantische Beschaffenheit $(2.2 .1,2.2 .2)$ und

bb) die Morphosyntax (Kategorie) dieser Objekte (2.2.3)

Um Frage b) beantworten zu können, bedarf es zunächst einmal einer Auseinandersetzung mit a).

\subsection{Verortung der es-Phorik im Kontext der Begriffe Referenz, Substitution und Deixis}

Obwohl das Charakterisieren von phorischem es mit Hilfe aller oben genannten Termini schließlich darauf hinausläuft, dass ein es angenommen wird, das semantisch, inhaltlich nicht leer ist (Admoni 1976: 222), muss darauf aufmerksam gemacht werden, dass diese Begriffe doch unterschiedliche Dimensionen darstellen. Zunächst sollen daher die genannten Grundbegriffe erklärt werden. Im Anschluss daran wird auf die möglichen Bezugsobjekte beim phorischen es eingegangen.

Grundsätzlich ist Referenz von den drei anderen Begriffen zu trennen. Unter Referenz versteht man den „Akt, mit dem auf eine bestimmte Wirklichkeit Bezug genommen wird“ (Duden-Grammatik 2005: 1146). Vater (2005: 11) gibt folgende Definition:

\footnotetext{
${ }^{1}$ Die Bezeichnung „Stellvertreter einer Nominalphrase“ in der Duden-Grammatik (2005: 830) ist praktisch die syntaktische Ausbuchstabierung der (syntagmatischen) Substituenshaftigkeit von es.
} 
In einer Äußerung wird explizit oder implizit auf die der Prädikation zugrunde liegende Situation sowie auf die von den Argumenten bezeichneten Orte, Zeitintervalle und Gegenstände Bezug genommen. Diese Bezugnahme wird ,Referenz ${ }^{`}$ genannt.

Im Wesentlichen handelt es sich bei der Erforschung von Referenz um drei Fragenkomplexe: Einerseits kann untersucht werden, wie Objekte, auf die referiert wird, beschaffen sind. Vater (2005: 71f.) spricht in diesem Zusammenhang von Situations-, Ding-, Ort- und Zeitreferenz. Wie auch aus obigem Zitat ersichtlich, kommen also vor allem Gegenstände, Zeiträume und Orte bzw. so genannte „abstrakte Objekte“ (Asher 1993), etwa Sachverhalte, Ereignisse, Tatsachen und Zustände (Vater 2005: 76, 80) in Frage. Zweitens können Referenzmittel, d.h. sprachliche Ausdrucksformen, mit denen referiert wird, den Gegenstand entsprechender Untersuchungen bilden. $\mathrm{Zu}$ denken ist dabei etwa an nominale, pronominale, verbale und noch komplexere Formen (Sätze). Dabei kommt es in erster Linie auf ihre lexikalische Spezifizierung und ihre grammatischen Merkmale an (Szakmary 2002: 17f.). Interessant ist auch die Korrelierung des ersten mit dem zweiten Fragekomplex, wobei geklärt werden kann, durch welche sprachlichen Referenzmittel auf bestimmte Objekte typischerweise Bezug genommen wird. Beispielsweise bietet sich die Korrelation abstrakter Objekte mit verbhaltigen, satzförmigen Ausdrucksformen an, was wiederum nicht heißen soll, dass andere Konstellationen nicht vorliegen können, vgl. etwa die so genannten „sentential nominals“ (z.B. ing-Gerundien wie throwing) in Asher (1993: 16) und ähnlich Nominalisierungen (wie z.B. Absage) in Vater (2005: 79f.). Drittens gilt es in der ReferenzLinguistik (Bezeichnung in Vater 2005) der Frage nachzugehen, wie Referenzialisierung (Szakmary 2002: 16) als Prozess beschrieben und interpretiert werden kann. Dieser Aspekt ist hauptsächlich aus kognitionslinguistischer Sicht relevant. Einschlägig für den theoretischen Hintergrund der vorliegenden Arbeit sind Pronomina als Referenzmittel. So referieren im Falle des Belegs

(1) Sobald ein Pferd von uns sturbe, welches gar oft geschahe, maßen wir gar keines behielten, tranchiereten sie es gleich und machten sich lustig dabei. (19) (MD)

sowohl die Nominalphrase „ein Pferd von uns“ als auch das pronominale es auf dieselbe außersprachliche Entität, den Referenten (auch: Bezugsobjekt, s. von Polenz 1985: 116), in 
diesem Falle auf ein Lebewesen. „ein Pferd von uns“ und „es“ sind in diesem Sinne referenzidentisch: ${ }^{2}$

Wenn ein Text einen lexikalischen Ausdruck verwendet, um auf einen thematischen Sachverhalt ein zweites Mal Bezug zu nehmen, spricht man von Referenzidentität. Der Text fordert den Leser damit auf, den Bezug der Begriffe zu einer gemeinsamen Wirklichkeit herzustellen. (Duden-Grammatik 2005: 1150)

Bei Szakmary (2002: 17, s. aber auch Vater 2005: 47) wird in diesem Fall von Koreferenz gesprochen: „Koreferenz liegt dann vor, wenn ein Textproduzent mittels zweier oder mehrere [sic!] Ausdrücke ein und dasselbe Konzept versprachlicht.“ Es stellt sich dabei die Frage, ob nominale und pronominale Ausdrucksformen auf die gleiche Art referieren, was bedeuten würde, dass auch Pronomina über eine eigene referierende Kraft verfügen, oder ob es sich bei pronominaler Referenz um eine vermittelte geht (Szakmary 2002: 12f.). Letztere Option sieht eine Referenzleistung von Pronomina vor, die erst durch den Rückbezug auf einen Vorgängerausdruck erbracht werden kann. Ob dem so ist, ist umstritten (ebd., 13). In der vorliegenden Arbeit wird der Akzent nicht auf die Referenzleistung von Pronomina, so auf die von es, sondern auf das phorische Verhältnis zwischen es und dem Bezugsausdruck, auf den es sich phorisch bezieht, gelegt. Im Falle der Begriffe Phorik und Substitution geht es also darum, die beiden Bezugswörter, die also beide auf den Referenten Bezug nehmen, aufeinander $\mathrm{zu}$ beziehen, während etwa Wiederbezug (von Polenz 1985: 116) gewissermaßen die beiden Bezugsarten, nämlich Referenz auf der einen und Phorik bzw. Substitution auf der andern Seite, vereint, s. dazu weiter unten.

Phorik bedeutet zunächst Verweisen. Auf Grund der Richtung des Verweisens werden Anaphern von Kataphern unterschieden. Da Kataphern ein Gegenstück zu Anaphern darstellen (IDS-Grammatik 1997: 547; Harweg 1979: 54³) und diese daher als begrifflicher Anhaltspunkt gelten können, ist es sinnvoll, Phorik ausgehend von der Definition von Anaphorik zu bestimmen. Vor diesem Hintergrund kann in einem zweiten Schritt auf Kataphern eingegangen werden. Anaphern sind

\footnotetext{
${ }^{2}$ Vgl. hierzu kritisch Diewald (1991: 119).

${ }^{3}$ Dort als „Anaphora“ und „Kataphora“ angeführt und mit einem Hinweis auf Differenzen zwischen echten und Pseudo-Kataphern. Auf diesen Aspekt wird hier nicht eingegangen.
} 
sprachliche Ausdrücke, mit denen ein Sprecher/Autor für die Beibehaltung der Orientierung auf bereits eingeführte oder sonst mental präsente Gegenstände oder Sachverhalte sorgt. Auf dem Wege der mentalen Verarbeitung werden Elemente der Vorgängeräußerung unmittelbar in die Folgeäußerung übernommen. (IDS-Grammatik 1997: 544, vgl. auch 37)

Dieser Definition ist zu entnehmen, dass Anaphorik ein prozeduraler Begriff ist. Es geht hier nicht um anaphorische Wörter sui generis, sondern um Ausdrücke, die der anaphorischen Prozedur dienen, d.h. für solche Zwecke eingesetzt werden (können). Bezeichnet man also das es im obigen Beispiel als (ana)phorisch, so bringt man dadurch sein syntagmatisches Verhältnis zu „ein Pferd von uns“ zum Ausdruck. In diesem Fall werden Ausdrücke, zu denen es in syntagmatischem Verhältnis steht, Antezedentien genannt (Pause 1991: 548). Ein solches es als Anapher ist „das einfachste Mittel, um thematische Kontinuität [...] zum Ausdruck zu bringen“ (IDS-Grammatik 1997: 544). Diese Art der Themafortführung - durch die thematische Kontinuität aufrechterhalten wird - wird als „anaphorische Prozedur“ bezeichnet und dient der ,inhaltliche[n] Verarbeitung eines Äußerungsteils in Relation zu dem, was vorausgegangen ist“" (ebd.). Spricht man also von phorischem es, so meint man damit einen Verweis, der ein inhaltliches Ziel hat, welches Ziel wiederum mit Hilfe eines sprachlichen Ausdrucks durch mentale Verarbeitung erreicht wird. Lässt man die Richtung des Verweisens weg, so bekommt man allgemein eine Begriffsbestimmung von Phorik. Anaphorik und die Leistung von Anaphern werden sowohl in der IDS-Grammatik als auch bei Harweg zu Grunde gelegt, wenn Kataphern und Kataphorik definiert werden. So heißt es in der IDS-Grammatik (1997: 548): „Kataphern nennen wir Gebrauchsformen der Anapher, die eine Orientierungskontinuität anzeigen, ohne daß eine vorgängige Orientierung erfolgt wäre“ bzw. „Insofern ist die Rede von ,Kataphern“ - statt von ,kataphorisch gebrauchten Anaphern“ - abkürzend.“ Harweg (1979: 54) formuliert: „Lediglich die Reihenfolge von syntagmatischem Substituendum und Substituens ist, wie bereits angedeutet, umgekehrt".5 In Schmidt (1987: 97) liest man des Weiteren: „Indem sie aber die normalen Verhältnisse umkehren, setzen kataphorische

\footnotetext{
${ }^{4}$ Ein wichtiger Bestandteil dieser Definition sind auch die Hinweise auf die mentale Verarbeitung phorischer Prozesse. Dabei geht es vor allem um den „Zugang“ etwa phorischer Ausdrücke zu ihren Antezedentien in der so genannten „accessibility theory“, s. z.B. Ariel (1990). Eine detaillierte Darlegung dieses Gesichtspunktes kann im Rahmen der vorliegenden Arbeit nicht geleistet werden, vgl. aber ausführlich dazu van Hoek (1997), Langacker (2007: 178) und Schwarz-Friesel/Consten/Marx (2004: 71) bzw. Szakmary (2002: 19), die „Interpretationsstrategie für Anaphern“ in der IDS-Grammatik (1997: 549) und in Pause (1991: 555).

${ }_{5}^{5}$ Auch Weinrich führt Kata- auf Anaphern zurück, indem er (1993: 373) von „Prä-Pronominalisierung“ spricht.
} 
Substituentia die anaphorischen Substitutionsverhältnisse gewissermaßen als Folie voraus.“ Hier scheint reine Spiegelbildlichkeit vorzuliegen, indem sich nur die Verweisrichtung ändert. Eine solche „Gleichsetzung“ von Ana- und Kataphern ist meiner Meinung nach problematisch. Rein theoretisch und definitionstechnisch dürfte es mit einer solchen „Folien“-Annahme stimmen, es wäre jedoch voreilig zu urteilen, Kataphern seien reine Umkehrungen von Anaphern. Denn zwischen ihnen besteht ein wichtiger funktionaler Unterschied, folglich muss auch die mentale Verarbeitung von Kataphern anders beschaffen sein (Eisenberg 2006: 168): Die Orientierung wird nämlich nicht aufrechterhalten, sondern vom Adressaten erwartet, antizipiert, wobei seine Aufmerksamkeit „,in besonderer Weise auf den thematisierenden Ausdruck gelenkt [wird]“" (IDS-Grammatik 1997: 548), vgl. etwa folgenden Beleg:

(2) es war eine Lust und Freude jeden Morgen das Auflesen unter den Bäumen und eine Lust, immer vom schönsten Obst zu essen. (17) (SCHG)

Dieses Beispiel für eine so genannte Rechtsversetzung zeigt, wie die es-Katapher durch einen Verweis auf den Rechtskontext dessen zentrales Thema vorwegnimmt. Außerdem ist aus dem Beleg ersichtlich, dass dieses kataphorische Verfahren ein zweites Mal, nämlich elliptisch, eingesetzt wird. Würde man hier ein zweites es explizit setzen $(, \ldots$ und es war eine Lust, immer vom schönsten Obst zu essen.“), so bekäme man ein Korrelat-es, das wiederum auf den kataphorischen Gebrauch zurückzuführen (IDS-Grammatik 1997: 549), jedoch syntaktisch gebunden ist (vgl. hierzu detaillierter Kapitel 2.3). Denkt man über Beispiele für Kataphern nach, so stellt sich die Frage, ob es denn tatsächlich kataphorisch gebrauchte Anaphern sind. In der Tat zeigen sie Orientierungskontinuität an, wenn man berücksichtigt, dass eine Beziehung zu einem anderen Ausdruck hergestellt wird. Aber das andere definitorische Merkmal, nämlich dass auf bereits Eingeführtes Bezug genommen wird, liegt gar nicht vor. Um Ehlich (2007: 43) zu zitieren: „Sie [die Anapher, D.C.] dient dazu, dem Hörer die Möglichkeit zu geben, ein einmal in den Fokus genommenes mentales Objekt als solches dort zu halten." Was heißt es also bei der Bestimmung von Kataphern, dass da Anaphern kataphorisch gebraucht werden, wenn ein wichtiger Definitionsbestandteil auf sie gar nicht zutrifft? Eine Antwort auf diese Frage wird bei der Darstellung syntaktisch gebundener Phorik, d.h. des Gebrauchs von es-Korrelaten, gegeben. 
Eine weitere Möglichkeit, Phorik $\mathrm{zu}$ fassen, liegt in der Herstellung eines Zusammenhangs mit dem Begriff der Substitution. Betrachtet man phorische Ausdrücke als (eine Art) sprachliche Mittel der thematischen Fortführung, so kann man den Harweg'schen Begriff der Substitution durch Pronomina als das Wie oder als eine Art ökonomische Ausbuchstabierung thematischer Fortführung ansehen. So definiert Harweg (1979: 20) Substitution als „die Ersetzung eines sprachlichen Ausdrucks durch einen bestimmten anderen sprachlichen Ausdruck“. Weinrich (1993: 372) bezeichnet das Wie der Themafortführung als Pronominalisierung:

Die Bedeutung von Textualität in Form von thematischer Konstanz im Text durch wörtliche Rekurrenz oder semantische Variation eines Nomens ist verhältnismäßig aufwendig. Einfacher und ökonomischer hält ein Sprecher die Thematik eines Textes dadurch konstant, daß er ein Nomen pronominalisiert.

Dabei sind anaphorische Pronomina grundsätzlich zweidimensionale syntagmatische Substituentia ihrer Substituenda (Harweg 1979: 25). Syntagmatisch heißt, dass zu Ersetzendes (Substituendum) und Ersetzendes (Substituens) nacheinander stehen (ebd., 20) und eine Identität aufweisen, die sich auf die „Bezeichnungskorrelate der Substitutionsausdrücke [nämlich den Referenten, D.C.]““ (ebd.) bezieht. Des Weiteren sind sie auch bezüglich des im jeweiligen Text aktualisierten Bezeichnungsumfangs identisch (ebd., 22f.). Diese Art von Substitution, nämlich die syntagmatische durch grammatische Funktionswörter, wird auch ein Mittel der Herstellung der Textkohäsion genannt (DudenGrammatik 2005: 1151). An dieser Stelle ergibt sich auch die Frage, ob denn Substituenda wie ihre Substituentia denotieren (Diewald 1991: 119). Diewalds Antwort darauf (ebd.) ist ein klares Nein: „Nicht der textphorische Ausdruck denotiert, sondern sein Antezedens, dessen Denotation er übernimmt.“ Sie argumentiert, dass Anaphern auf einen bereits denotierenden Ausdruck Bezug nehmen. Es handele sich bei Anaphern also nur um eine vermittelte Denotation und es sind Deixeis, die direkt denotieren, so Diewald (ebd.). Diese Argumentation hat zur Folge,

daß die Beziehung des textphorischen Ausdrucks und seines Vorgängerausdrucks zum Denotat nicht als "Koreferenz" (im Sinne von "Ko-Denotation") bezeichnet werden kann, wenn man $[\ldots]$ davon ausgeht, daß der textphorische Ausdruck nicht selbst denotiert. (ebd.) 
Die Frage, ob Diewalds Position oder diejenige, nach der Anaphern selber denotieren, zu befürworten ist, kann und soll im Rahmen der vorliegenden Arbeit nicht beantwortet werden. Bezüglich der Festlegungen zur es-Phorik spielt es m.E. keine entscheidende Rolle, ob pronominale Anaphern semantisch zur Denotation aus eigener Kraft fähig sind oder dies nur vermittelt tun können. ${ }^{6}$ Oben ist kurz angedeutet worden, dass anaphorische Pronomina zweidimensionale syntagmatische Substituenda sind. Zweidimensional steht dabei für die Komplexität des Funktionierens anaphorischer Pronomina, nämlich dass sie imstande sind, über die syntagmatische Substitution vermittelt auch paradigmatisch zu substituieren. Dies besteht darin, dass sie während der Substitution auch eine vom aktuellen Text unabhängige Klassenbedeutung tragen (Harweg 1979: 23). Diese Variante der Ersetzung eines sprachlichen Ausdrucks durch einen anderen wird in der Duden-Grammatik (2005: 1150f.) der Textkohärenz zugeordnet. Mit Harweg (1979: 21) wird im Folgenden davon ausgegangen, dass für Textkonstitution und Themafortführung der Begriff der syntagmatischen Substitution einschlägig ist. Dementsprechend wird Substitution in diesem engeren Sinne verwendet. ${ }^{7}$

Obwohl eine Zusammenführung von Phorik und Substitution auf der Hand liegt, so muss man doch darauf hinweisen, dass es wohl möglich ist, diese beiden Termini gerade unter Bezugnahme auf es-Typen auseinanderzuhalten, wie Schmidt in seiner Monografie zu Impersonalia (1987) dies tut. Unter Rückgriff auf Harwegs Festlegungen zur syntagmatischen Substitution geht er der Frage nach, wie Belege der Art

(3) da werden die Pfingsten schöner sein, es werden vielleicht die Lieben aus Teplitz kommen. (5) (K)

(4) Aber diese Bezeichnungen machen es ja eigentlich deutlich, wie man zu diesen Leuten steht (13) (SB)

zu interpretieren sind, ob also das es in diesen Fällen überhaupt als phorisch gelten soll. Es handelt sich dabei um das oft als expletiv bezeichnete, satzeröffnende (vgl. (3)) und um das Korrelat-es (vgl. (4)). Schmidt kommt zu dem Schluss, dass es in Beispielen der obigen Art zwar eine gewisse „kataphorische Kraft“ besitzt (1987: 97), jedoch kein Pronomen darstellt

\footnotetext{
${ }^{6}$ Des Weiteren soll auf die Frage, ob Denotation und Referieren gleichzusetzen sind, aus dem gleichen Grund nicht eingegangen werden, vgl. aber Vater (2005: 42).

${ }^{7}$ Dieser textbasierte Begriff der Substitution hat des Weiteren nichts zu tun mit dem generativen SubstitutionsAnsatz, vgl. hierzu Consten/Schwarz-Friesel (2007: 272f.).
} 
(ebd., 98). Dies heißt vor dem Hintergrund der Harweg'schen Begriffsbestimmung von Pronomina, dass es nicht substituiert, weil

a) Kongruenzbeziehungen zwischen es und dem folgenden morphologischen Subjekt aufgehoben werden, ${ }^{8}$

b) „ES [Hervorhebung im Original, D.C.] und der folgende Ausdruck nicht Glieder einer Substitutionskette "Substituens-Substituendum" sind“69 und

c) die Kataphorizität nicht die Umkehrung eines anaphorischen Verhältnisses darstellt. (ebd.)

Die Gültigkeit von b) und c) erkennt man auch daran, dass das es dieser Beispiele rechts von dem Ausdruck, auf den es zeigt, gar nicht stehen kann (ebd., 97):

* da werden die Pfingsten schöner sein, vielleicht werden es die Lieben aus Teplitz kommen.

* Wie man zu diesen Leuten steht, machen es diese Bezeichnungen ja eigentlich deutlich.

Aus oben genannten Gründen trennt Schmidt (ebd., 107) Substituenshaftigkeit und Phorik von es und bezeichnet das expletive und das Korrelat-es als zwar phorisch, aber nicht substituenshaft. So gesehen ist Phorik weniger als Substitution, da Letztere bei Ersterer nicht vorzuliegen braucht. M.a.W.: Substituierendes es ist gleichzeitig phorisch, nicht aber umgekehrt. Die IDS-Grammatik (1997: 549) macht auf diese Sonderstellung beim Korrelat aufmerksam, indem sie es zwar auf den kataphorischen Gebrauch zurückführt, dabei jedoch, da das Korrelat syntaktisch gebunden ist, nicht von Kataphorik spricht. Man könnte sagen, dass die Position Schmidts insofern eine textlinguistische Weiterentwicklung dieses

\footnotetext{
${ }^{8}$ Der Ausdruck „die Lieben aus Teplitz“ ist eine pluralische Form, der Subjektsatz verfügt über gar keine nominalen, Kongruenz stiftenden Eigenschaften.

${ }^{9}$ „Das ES weist lediglich auf den folgenden daß-Satz hin; denn der Ausdruck Aber diese Bezeichnungen machen es ja eigentlich deutlich [Originalbeispiel ersetzt durch eines aus meinem Korpus, D.C.] ist keine vollständige Aussage. Das ES ist keine semantisch gesättigte Form; es ist nicht, wie jedes nominale Substituens, eine - qua Koreferentialität mit dem Substituendum-Ausdruck - "autonome Form" [Hervorhebungen im Original, D.C.]“ (ebd.).
} 
syntaktisch orientierten Gedankens ist, ${ }^{10}$ als Phorik im Falle des Korrelates nicht zur Textkonstitution beiträgt und in diesem Sinne sich der Substitution entzieht.

Eine Auseinandersetzung mit Phorik und Substitution erfolgt gewöhnlich im Kontext einer Diskussion über Deixis. Dieser Aspekt, d.h. Gemeinsamkeiten und Unterschiede zwischen Phorik und Deixis, können hier nicht in allen Details besprochen werden, daher werden nur die für vorliegende Arbeit relevanten Aspekte thematisiert. Auch Deixis stellt, wie Phorik, eine Art Bezugnahme dar. Beide sind Formen des Zeigens (Verweisens): „Auch der phorische Gebrauch von Pronomina kann als textverweisend und damit als textdeiktisch angesehen werden, daher spricht Bühler von anaphorischer Deixis.“ (Eisenberg 2006: 171) Aber während für die Spezifikation der Bedeutung eines anaphorischen Elements „keine außersprachliche, situationsimmanente Zeighilfe wie Gesten usw. vonnöten ist“" (Harweg 1979: 43), sind solche außersprachlichen Zeighilfen im Falle deiktischer Prozeduren unentbehrlich. In diesem Sinne enthält die deiktische Prozedur ein spezifisches Plus gegenüber Phorik, bei der „Funktionswörter lediglich Schlüsse über den Bezug zu Inhalten auslösen, ohne explizit zu zeigen“ (Duden-Grammatik 2005: 1114). Ein explizites Zeigen bedeutet die Orientierung der Aufmerksamkeit des Adressaten auf bestimmte Punkte oder Bereiche „eines unmittelbar präsenten oder konstituierbaren Verweisraums“ (IDSGrammatik 1997: 311, s. auch Eisenberg 2006: 170). In diesem Fall liegt der Bezugspunkt direkt beim Sprecher. Demgegenüber wird beim phorischen Zeigen ein solcher Bezugspunkt im Text gesetzt (Duden-Grammatik 2005: 583), m.a.W.: „Als Faustregel zur Abgrenzung von Deixis und Anaphorik hat sich bewährt: Der Bezug auf etwas, was vorher im Text schon einmal vorkam, ist anaphorisch.“ (Ágel/Hennig 2006: 57, vgl. auch Bühler 1934: 121) In Anlehnung an die Ausführungen in der IDS-Grammatik (1997: 311 bzw. 544) kann man etwas vereinfachend formulieren: Bei der Deixis liegt der Schwerpunkt auf dem direkten (Neu)Orientieren, ${ }^{11}$ während Phorik in erster Linie der Beibehaltung der Orientierung auf bereits Eingeführtes dient. ${ }^{12}$ Während für Bühler (1934) sowohl phorisch als auch deiktisch verwendete Ausdrücke als Zeigwörter galten, also eine einheitliche Klasse zeigender sprachlicher Mittel postuliert wurde, wird in der späteren Literatur, so in Grammatiken und bei Harweg (1979), Diewald (1991) oder Ehlich (2007), stärker auf

\footnotetext{
${ }^{10}$ Wenn man einmal davon absieht, dass Schmidts Monografie acht Jahre früher entstand.

${ }^{11}$ Vgl. auch Eisenberg (2006: 171): ,[...] eben weil die deiktische Prozedur als Neuorientierung des Adressaten auf ein Objekt begriffen wird.“"

${ }^{12}$ Vgl. hierzu Cornish (2005: 201f.) sowie Ehlich (2007: 22f.): „Der Rückbezug [durch eine Anapher, D.C.] teilt dem Hörer vielmehr mit, daß eine vorgängige Fokussierung als solche beibehalten werden kann und soll. Insofern ist der Rückbezug ein Kontinuitätssignal, das der Sprecher dem Hörer gibt. [Kursivierungen im Original, D.C.]“"
} 
Differenzen abgehoben. Etwas komplizierter wird das Bild dadurch, dass auch der Begriff der so genannten Textdeixis auftaucht (Harweg 1979: 167, Diewald 1991: 110, 121, Ehlich 2007: 15). In der IDS-Grammatik (1997: 539ff.) heißt es, dass beide Prozeduren, Textphorik und Textdeixis, der Themafortführung und Themenentwicklung in Texten dienen. Die Parallelität wird u.a. dadurch sichtbar, dass - wie im Falle der Phorik - auch bei der Textdeixis zwei funktionale Subklassen angenommen werden, die die beiden möglichen Richtungen des Zeigens widerspiegeln: Ana- und Katadeixis (ebd., 314, s. auch DudenGrammatik 2005: 1114). Andererseits (ebd., 558ff.) werden jedoch wiederum wichtige Differenzen herausgestellt und es wird festgehalten:

\begin{abstract}
Auch wenn Anapher und Anadeixis beide der thematischen Fortführung dienen können, so tun sie dies doch auf unterschiedliche Weise und sind nicht immer durch die jeweils andere Form ersetzbar. Eine Anapher kann ja nur verwendet werden, wenn das Thema schon präsent ist, also deiktischer Neuorientierung nicht bedarf. Sie wird insbesondere dann verwendet, wenn etwas bereits in der Vorgängeräußerung Thema ist. (ebd., 558, s. auch Consten/Schwarz-Friesel 2007: 269)
\end{abstract}

Ehlich (2007: 15f.) führt aus, dass es trotz der möglichen Analogie ein entscheidender Schritt ist, wenn aus Reden Texte entstehen und dabei ein Textraum als Verweisraum fungiert. Dadurch kommt es nämlich zu der „Situationsentbindung des Textes“ (ebd., 17). Darüber hinaus gilt: Um sprachliche Ausdrücke anadeiktisch zu gebrauchen, bedarf es zusätzlicher bzw. anderer Bedingungen wie bspw. erster Thematisierung vor der Anadeixis, der Zugehörigkeit des Bezugsobjektes zum Rhema oder der Konkurrenz mit anderen Themen (IDS-Grammatik 1997: 558). Als Zwischenfazit lässt sich in Anlehnung an Diewald (1991) und Ehlich (2007) Folgendes sagen:

Deixis und Phorik sind beide Modi des Zeigens (Diewald 1991: 110). ${ }^{13}$ Sie sind „gerichtete Relationen“ (ebd., 117), d.h. es wird von einem Ort auf einen anderen gezeigt.

\footnotetext{
${ }^{13}$ Diewald (1991: 118) unterteilt Deixis weiter in Realdeixis, Deixis am Phantasma (übernommen aus Bühler 1934) und Textdeixis.
} 
Beide haben jedoch unterschiedliche Zeigfelder. Bei der Deixis „ist die gerichtete Relation auf den außersprachlichen Kontext bezogen“ (ebd.), während Phorik „ein textinterner Prozeß [ist], der nicht vom Äußerungskontext abhängig ist.“ (ebd., 120).

Entscheidend bei der Unterscheidung von Phorik und Deixis ist, dass Letztere eine Orientierung oder erste Fokussierung auf Elemente im Verweisraum bedeutet, während Erstere einen Rückbezug auf bereits Verbalisiertes oder Fokussiertes, d.h. Bekanntes, herstellt (Ehlich 2007: 20f., 23).

Für die vorliegende Arbeit stellt sich hier die Frage, ob das „zeigende“ es phorisch, deiktisch oder beides sein kann. Diese Frage wird in zwei Teilbereiche geteilt:

- Korrelation zwischen es und das

- Bezug auf Mitbedeutetes (von Polenz 1985) durch es

An dieser Stelle soll nur der erste Punkt besprochen werden, auf den zweiten gehe ich in 2.2.1 ein. Den Überlegungen zum so genannten „Fokuspronomen“ in Weinrich (1993: 401406), den Festlegungen in der IDS-Grammatik (1997: 555) und Zifonun (2001: 92,111 und 115f.) folgend lässt sich die Faustregel formulieren, dass es als grundsätzlich schwaches Pronomen phorisch verwendet wird, während bei der deiktischen Prozedur vornehmlich die Formen dies(es) und das Anwendung finden (s. auch die IDS-Grammatik 1997: 1475). Auch Diewald (1991: 121) weist darauf hin, dass drittpersonige Personalpronomina „bis auf das Neutrum "es"“، deiktisch verwendet werden können. In Anlehnung an Weinrichs Ausführungen (1993: 401-405) und Bethke (1990: 182) kann das in Kontrast zu es die Funktion Fokussierung zugeschrieben werden. Weinrich (1993: 401) hebt die „Auffälligkeit“ von das hervor: „Das Fokus-Pronomen das ist das auffällige Gegenstück zum unauffälligen Horizont-Pronomen $e s^{\star}$, vgl. auch Behaghel (1923: 327ff.). Erfüllt das eine zeigende Funktion, so erweist sich hier qua seiner Stärke ein deiktischer Verweisrahmen als besonders günstig, da ja durch Fokussierung besonders gut orientiert werden kann, was wiederum das zentrale Merkmal deiktischer Prozedur ist. Solche Fokussierungen können auf verschiedenste Weise zum Ausdruck gebracht werden, sodass die deiktische Kraft von das viele „es-Gebiete“ erfassen kann, darunter auch folgende: 
(5) Gerti [...] Bertha [...] Es sind halt überhaupt 2 wilde Kinder, wo sie das nur her haben?????? (1890) (LB)

vs.

Die hätten damals diesen anbau dazu nehmen müssen und dann die fleischabteilung daraus machen hm hör mal sollen das nicht geputzte pilze sein? (Beispiel aus Stein 1995: 207)

(6) 〈Weisst Du, später, wenns Du einmal weisst, wie mans macht, musst Du allein die Gänse treiben. (9) (SCHG)

vs.

Aber das kan ich dir sagen, mich hörst du seither kein Wort nicht schelten, seit dem Starrkrampf. (Beispiel aus Hegedüs 2007: 260)

(7) Es regnet.

vs.

Wie das heute wieder regnet! (Beispiel aus Weinrich 1993: 403, vgl. auch Auer 1993: 195)

In (5) steht der Identifizierungskonstruktion (Askedal 1990: 214) eine ,auffällige Identifikation“ (Weinrich 1993: 401) gegenüber. Die Setzung von deiktischem das signalisiert hier die Rückkehr aus dem Monolog in den Dialog und dabei in den unmittelbar präsenten Verweisraum. Das zweite Glied des Paares (6) zeigt ein Beispiel für die so genannte Katadeixis, die hier stark auf das Folgende orientiert und dieses fokussiert. Genau diese Differenz zwischen das und es im Allgemeinen (d.h. bezogen auch auf Ana-Fälle) wird des Öfteren als operationales Kriterium verwendet, um es-Typen, die eine Ersetzung durch das erlauben, von denen ohne diese Option zu trennen, vgl. etwa Pütz (1975: 35) oder Askedal (1990: 213). (7) stellt die Fokussierung des „Naturhorizontes“ (Weinrich 1993: 391) durch das dar: „In das liegt eine wirkliche Deixis; ob eine deiktische Geberde zum sprachlichen Ausdruck hinzukommt oder nicht, ist gleichgültig. In es regnet hingegen besagt es für sich gar nichts.“ (Brugmann 1917: 9). Dieses und die anderen Beispiele oben, deren es konstruktionell-syntaktisch, vgl. (5) und (6), oder semantisch, vgl. (7), gebunden ist, wobei eine Austauschbarkeit durch das jedoch wohl gegeben ist, sprechen dafür, dass die Möglichkeit der Ersetzung von es durch das nicht als syntaktisches Kriterium zur Etablierung scharf getrennter es-Klassen $\mathrm{zu}$ Grunde gelegt werden, sondern als 
grundsätzlich verfügbares deiktisches Ausdrucksmittel (gegebenenfalls für es-dasKontraste) gesehen werden sollte. ${ }^{14} \mathrm{Da}$ eine ausführliche Darstellung dieses Kontrastes, unterstützt durch empirische Daten, den Rahmen der vorliegenden Arbeit sprengen würde, wird hier auf die weitere Diskussion verzichtet. Ein guter Überblick hierüber findet sich in Behaghel (1923: 327ff.) vgl. auch die Verwendung von dies/das als Korrelate (IDSGrammatik 1997: 1480).

Mit Bezug auf die Unterscheidung zwischen Phorik und Referenz lohnt sich schließlich noch ein Blick auf den Begriff Wiederbezug bei Peter von Polenz (1985). Die Definierung dieses Terminus nimmt er (1985: 116) im Zusammenhang der Begriffsbestimmung von Bezugnehmen vor:

Immer wenn man eine Prädikation/Aussage macht, muß es etwas geben, vorüber man das Prädikat aussagt. Dieses Etwas ist aber nicht von vornherein in der außersprachlichen Wirklichkeit gegeben; es muß vom Sprecher/Verfasser im Zusammenhang mit seiner Aussage satzsemantisch konstituiert werden, ist Objekt einer Teilhandlung des Satzinhalts.

„Normalsprachlich“ (ebd., 117) kann dieser Akt als Bezugnehmen bezeichnet werden. Diese Definition der ersten Bezugnahme bei der Konstituierung von Satzinhalten kann den Ausgangspunkt weiterer Bezugnahmen bilden, die dazu beitragen, einen Textzusammenhang herzustellen:

Den Bereich des offensichtlichen Textzusammenhangs [...] bilden die Möglichkeiten, innerhalb des Textverlaufs sich auf schon erwähntes WIEDERZUBEZIEHEN. Man kann ihn einfach Rereferenz/Wiederbezug nennen, da es sich konkret um Wiederholungshandlungen im Textverlauf handelt (wenn man nur die reine Referenzhandlung berücksichtigt). In der Forschungsliteratur wird er meistens „Textreferenz“"genannt, abstrakter manchmal „Textphorik“. (von Polenz 1985: 138)

Aus dem Zitat ist ersichtlich, dass es hier in erster Linie um Referenz geht, dabei jedoch auch der andere Aspekt angesprochen wird, nämlich dass durch eine erneute Bezugnahme auf ein Bezugsobjekt der außersprachlichen Wirklichkeit ein Verhältnis zwischen erstem (Antezedens) und zweitem (und eventuell weiteren) Bezugsausdruck (Anapher) gestiftet ${ }^{14}$ Damit steht im Einklang, dass das und dieses nicht nur als Pro-Formen, sondern auch als Artikel bei Nomina der Deixis dienen können, vgl. Eisenberg (2006: 170). 
wird, das im Dienste des Textzusammenhangs steht. ${ }^{15}$ In diesem Sinne ist oben behauptet worden, dass die von Polenz'sche Herangehensweise gewissermaßen phorische und referenzielle Gesichtspunkte vereint. Trotz dieser engen Verknüpfung der beiden Begriffe bleibt klar, dass es sich um unterschiedliche Phänomene handelt, da, wie bereits erwähnt, Referenz das Verhältnis des ersten und zweiten auf einen Referenten Bezug nehmenden Ausdruckes spiegelt, während Phorik zum Ausdruck bringt, dass der erste und der zweite Bezug nehmende Ausdruck auch zueinander ein Verhältnis aufbauen. M.a.W.: Setzt man Referenz und Phorik - etwa terminologisch, indem man phorisch und referenziell synonym verwendet - gleich, so vermischt man die Referenzebene mit der Sprachebene eines Textes (Schwarz 2000: 53). Dass Referenz und Phorik auseinander gehalten werden sollten, kann am Beispiel von es regnet gezeigt werden. Hier dient es keinesfalls der Beibehaltung der Orientierung auf bereits Eingeführtes in Texten, es besitzt also keine textkonstituierende Kraft. Man kann auch darauf hinweisen, dass dieses es referenzlos oder referenziell leer (Zifonun 2001: 76) ist, weil es auf keinen Referenten (in der außersprachlichen Welt) verweist. Beides kann als gültige Aussage erachtet werden, sie heben jedoch auf jeweils andere Besonderheiten von es ab. Dass man sich dies vor Augen hält, erweist sich deshalb als nützlich, weil darüber diskutiert werden kann und wurde, ob das es in Witterungsimpersonalia doch referenziell ist, was bedeuten würde, dass eine übernatürliche Größe (etwa Gott) als Referent gesetzt werden kann, auf den Bezug genommen wird (vgl. etwa Behaghel 1923: 318, ${ }^{16}$ aber auch den Begriff des so genannten „situativen Horizonts“ bei Weinrich 1993: 391-396 und Back 1995: 162 ${ }^{17}$ ). Folgt man diesem „Tabuisierungskonzept“ (Schmidt 1987: 92), so folgt daraus bei Weitem nicht, dass dieses es auch phorisch ist. Eine syntagmatische Substitution eines vorangehenden Ausdruckes findet nämlich nicht statt und ein syntaktisch motivierter Verweis (wie bei Korrelaten) ist auch nicht vorhanden. ${ }^{18}$ Noch bedeutender wird die saubere Trennung des Redens von nicht-referenziellem und des von nicht-phorischem es, wenn man so genannte „okkasionelle

\footnotetext{
${ }^{15}$ Vgl. auch Consten/Schwarz-Friesel (2007: 265f.): „Anaphern sind Ausdrücke, die innerhalb eines Textes einen anderen Ausdruck (das Antezedens oder der Antezedent) [Kursivierungen im Original, D.C.] wieder aufgreifen und mit denen ein Sprecher somit auf einen im Text oder Diskurs bereits erwähnten Referenten erneut Bezug nimmt.“

${ }^{16}$ Die gleiche Art von Bezugnahme wird - freilich in etwas modernerer Form und in teilweise noch verallgemeinerterer Weise - auch für englisches impersonal it in Langacker (2007: 180) angenommen: „We can reasonably think of it as designating the relevant scope of awareness for whatever is at issue. With meteorological predicates (e.g. It's raining oder It's hot), it tends to be interpreted as the surrounding atmospheric enviroment. [Kursivierungen im Original, D.C.]“"

${ }^{17}$ Back spricht von ,diffuser Deixis“.

${ }^{18}$ Auch Backs Begriff der diffusen Deixis bezeichnet nicht den Bezug auf einen Vorgängerausdruck im Text, sondern den auf einen außersprachlichen Referenten.
} 
Ereignisverben“ des Typs es klopft/riecht mit berücksichtigt (Näß1 1996), vgl. etwa folgenden Beleg:

(8) Und da finden wir in dem ausgestorbenen Haus: Speck, Butter, Mehl, Eier, Fleisch und alles gnung. So er auch wohl gewußt. Da ging es mit uns an ein Sieden und Braten, denn wir wurden gar knapp erhalten (7) (MD)

Im Zusammenhang mit Beispielen dieser Art kann sehr wohl darüber diskutiert werden, was es heißt, dass es einen unpersönlichen Gebrauch habituell persönlich verwendeter Verben gibt (Näßl 1996: 41f.) oder wie denn ein Personalpronomen impersonal benutzt werden kann (Langacker 2007: 179) und ob es in diesen Fällen tatsächlich semantisch leer ist oder es sich dabei eher darum handelt, dass durch das neutrale Pronomen maximale Vagheit und Nicht-Begrenztheit erreicht werden (ebd., 180 und 2009, vgl. dazu auch die Überlegungen im Rahmen der Cognitive Grammar von Langacker in Smith 2002, 2005). Auch wenn also der Status solcher es-Vorkommen bezüglich Impersonalität umstritten sein mag, so ändert dies nichts daran, dass hier keine textkonstituierende Funktion im Sinne der Themafortführung vorliegt, von Phorik also nicht die Rede sein kann.

Zusammenfassend wird festgehalten:

- In Anlehnung an Harwegs These zur textkonstituierenden Rolle von Pro-Formen (1979) und die Ausführungen zur thematischen Fortführung in Texten in der IDSGrammatik (1997) wird der Schwerpunkt auf Phorik gelegt, was signalisieren soll, dass es für den Textzusammenhang eine bedeutende Rolle spielt. Hervorgehoben wird also das Verhältnis zwischen es und seinem Antezedenten, nicht das dieser beiden zu einem außersprachlichen Objekt. Die Referentialität von es, d.h. seine eventuelle Verbindung mit einer außersprachlichen Einheit, wird von Phorik getrennt und soll bei der Behandlung des nicht-phorischen es thematisiert werden.

- Daher stehen Wörter wie Antezedens, Bezugsobjekt, -ausdruck, -glied, -element usw. bzw. Bezug nehmen, sich beziehen auf etw., etwas wiederaufnehmen usw. für das phorische Verhältnis zwischen dem Substituens-es und seinem Substituendum. 
- Als phorisch gilt ein es-Vorkommen dann, wenn die oben beschriebene Art von Verweis vorliegt, d.h. ein Bezugsausdruck im Text identifiziert werden kann. Als phorisch gelten sollen weiterhin Fälle eines syntaktisch gebundenen und die eines so genannten impliziten Verweises, vgl. hierzu die nachfolgenden Kapitel. Nichtphorisch ist es, wenn weder explizit, noch implizit oder syntaktisch gebunden verwiesen wird.

\subsection{Semantische und morphosyntaktische Bestimmung der es-Phorik}

Am Anfang des vorangehenden Kapitels wurde kurz angedeutet, dass während der Beschreibung der möglichen Bezugsobjekte beim phorischen es Semantik und Form methodisch getrennt werden, d.h. einerseits die Beschaffenheit der Bezugsobjekte auf semantischer Basis, andererseits die kategoriale Realisierung dieser Bezugsobjekte unter Rückgriff auf (meist) herkömmliche morphosyntaktische Begriffe dargestellt wird. Das ergibt eine Unterteilung des Vorgehens in zwei Schritte:

Semantische Beschreibung der Bezugsobjekte (2.2.1 und 2.2.2)

Kategoriale Beschreibung der Bezugsobjekte (2.2.3)

Die Motivation hierfür stellen erhebliche empirische Schwierigkeiten bei der Analyse von es-Gebrauchsweisen und ihrer Klassifizierung dar. In Abhängigkeit von den jeweiligen Kriterien, die man einer Klassifikation zu Grunde legt, entsteht immer wieder eine andere Anzahl von es-Typen (vgl. hierzu stellvertretend Pütz 1975, Cho 1975, Admoni 1976, Leys 1979, Buscha 1988, Askedal 1990 und diverse Grammatiken, etwa die Duden-Grammatik 2005: 830f., Eisenberg 2006: 175-178 oder die IDS-Grammatik 1997: 38, 1082f.). Hinzu kommt, dass man im Falle vieler es-Typen oft auf semantische, syntaktische oder pragmatische Parallelen, Analogien stößt, was zur Folge hat, dass man ein bestimmtes esVorkommen mehreren Typen zuordnen kann (Admoni 1976: 222). Wie Admoni (ebd.) bereits klar gesehen hat, sieht das Ganze eher wie ein komplexes Gebilde mit wichtigen Eckpunkten und Kanten zwischen diesen Eckpunkten aus. Dabei sind die Kanten 
unterschiedlicher Natur. Die methodologische Entscheidung, die Form der Bezugsobjekte von deren semantischem Charakteristikum zu trennen, hat m.E. folgende Vorteile:

Die Komplexität des Funktionierens von phorischem es erscheint auf transparente Weise, indem beide Seiten der Medaille, d.h. sowohl die satz- und textsemantische Vielfalt der es-Bezugsobjekte als auch die formal-grammatische Realisierung der Bezugnahme sichtbar werden.

Durch die Verbindung der Bezugsobjekte mit den Kategorien können prototypische Konstellationen hergestellt werden, wodurch die Hauptlinie des Systems erkennbar wird, ohne dass weniger prototypische Kombinationen verdeckt würden.

Damit soll nicht behauptet werden, kriterienbasierte Kategorisierungen wären nicht sinnvoll oder irrelevant. Schließlich kann man ja auch die Merkmale Phorik liegt vor/liegt nicht vor, semantische/syntaktische Art des Bezugsobjektes, Art der Valenzsimulation (beim nichtphorischen es, s. hierzu Kapitel 3) als Kriterien betrachten.

Sowohl bei der semantischen als auch bei der formalen Beschreibung des es-Phorik werden Stufen postuliert. Dabei sollen diese Stufen auf einer Skala der Einengung angelegt werden. Einengung bedeutet zunächst, dass man auf der semantischen Seite vom Allgemeinen, inhaltlich Umfassenden zum Konkreten, auf der formalen Seite von Texten über Sätze zu Phrasenteilen übergeht. Die Annahme von Stufen erfolgt unter Rückgriff auf Wladimir Admonis Formulierung „Abnahme der konkreten Dinglichkeit“ (1976: 220) und stellt einen Versuch ihrer Operationalisierung dar, wobei die Formulierung „Abnahme“ entsprechend dem Begriff Einengung in „Zunahme der konkreten Dinglichkeit“ umgewandelt wird. Zwar definiert Admoni weder, was Dinglichkeit und deren Abnahme exakt bedeuten, noch spricht er dabei von Stufen, man kann seinen Beispielen und Ausführungen jedoch entnehmen, dass es dabei um die Frage geht, auf was für Objekte durch es verwiesen wird und wie unterschiedlich diese Bezugsobjekte in semantischer wie syntaktischer Hinsicht sein können bzw. dass es dabei um verschiedene Ausprägungen der Dinglichkeit geht. Um diese Verhältnisse darzustellen, werden hier Belege aus meinem Korpus angeführt und es wird auf die von Admoni angenommenen, nummerierten Gebrauchsweisen des es $(\mathrm{GW})$ in Klammern hingewiesen: 
(9) Der starke Zug nach den Mühlen hätte mich schier ertränket und ersauft, wann nicht mein Berkaner Mantel Wind gefasset und mich oben erhalten. Unter der großen Arbeit, wieder heraus zu kommen, war nichts als: JEsus, JEsus, ach JEsus, mein GOtt, hilf mir! mein Gedanke. Endlich war ein Stück Bauholz mit eingefroren. Daran half ich mir wieder raus. Da war kein Mensch, der es gesehen oder mir helfen können. (10) (MD) (vgl. GW 4)

(10) daß es der ewige Schlaf ist, den sie schläft, daß sie - kaum erwachen werde. Und so war es gekommen. Sie - ist um $6 \mathrm{Uhr}$ eingeschlafen, hat bis Mitternacht noch geathmet, u. ist dann verschieden. (24) (K) (vgl. GW 5)

(11) als aber ein bar stunden vergingen, und ich mich den lieben Gott befohlen mit gebet, und ich mich drein ergeben, ich württe die nacht tauren müßen, endlich hörede etwas gantz dunckel bochen, ich lag aber stille, es bocht noch 2 oder 3 mal, so dachte ich, es were ein gespenst, endlich als es nicht wolte auff hören, und recht starck anschlug, so kroch ich heraus, suchte die treppe und kroch rund um biß ich nauf kam. (26) (NE) (vg. GW 7)

(12) Endlich wie es bald zweÿ wolte schlagen das auch der Herr Graff lang weg war (28) (NE) (vgl. GW 8)

(13) Endlich entschloß ich mich, selber eins zu bauen, und sagte es meiner Schönen. Sie war's zufrieden, und bot mir wieder Geld dazu an. (11) (B) (vgl. GW 9)

(14) In dem obbeschriebenem Jahr ist das Obs an den Bä[u]men gantz verdorben, das wir in unseren Garten nicht ein Hutt voll bekomen haben, es seyen gewest Äpfel oder Birn. (31) (BL) (vgl. GW 10)

(15) durch seine ränke und gedungene guten freunde Brachte er Es würklich so weit beij seinem Vater | das er Ihn zum stamherrn machte (4) (DM) (vgl. GW 11A)

(16) die zeit verstrich, Es kahm kein schwager und kein kaufbrif (8) (DM) (vgl. GW 12) 
Nach Admoni (1976: 222)

sind nicht nur die GW 4, 5, 7, 8 durch die abnehmende konkrete Dinglichkeit miteinander verbunden, sondern auch die GW 9, 10 drücken verschiedene Arten solcher Abnahme (oder Verwischtheit) aus, und in GW 11A, 12 steigert sich solche Entdinglichung bis zur vollständigen semantischen Leere der Wortform es.

Admonis Hinweis auf mögliche Verbindungen zwischen einzelnen es-Gebrauchsweisen ist in erster Linie semantischer Natur, wie dies aus dem letzten Teil des Zitates hervorgeht. Am Rande $^{19}$ werden aber auch grammatisch motivierte Überlegungen sichtbar (so z.B. ebd., 221). Offensichtlich geht es hier darum, dass ein gewisses es-Bezugsobjekt semantisch anders aussieht als ein anderes, wobei dieser Unterschied auch mit grammatischen Eigenschaften der Bezugsglieder korreliert. So finden wir in (9) eine ganze Textstelle, auf die durch es Bezug genommen wird, und ähnlich ist es auch in (10), in dem allerdings ein kataphorischer Verweis vorliegt. ${ }^{20}$ Hinsichtlich (11) kann hervorgehoben werden, dass dabei durch es eine später aufzulösende Unbestimmtheit zum Ausdruck gebracht werden soll: „Gewisse Vermutungen über seine sachliche Bezogenheit können nahegelegt werden, und dann kommt es zu einer vollständigen Konkretisierung.“ (ebd., 220), vgl. die Fortsetzung im Text:

da war der Man wider, der Herr cantzler hätte auff meiner schwester bitte ihm befohlen, mich heraus zu thun, und ihr befohlen, sie solde mich mit in ihr hauß nehmen, und ihr beÿ 5 Reichsthaler straffe auferlegt das sie mich nicht aus dem hause liese den ich ginge in die heiser, und verführete die leute, und morgen punct 9 ur solt ihr wider im consistorio erscheinen. (26) (NE)

Bei (12) würde Admoni (ebd.) von unpersönlichem Subjekt sprechen, in (13) von „Bestimmung des prädikativen Adjektivs“. Auch die letzten drei Belege (14)-(16) repräsentieren nach seiner Meinung andere Gebrauchsweisen, es sind der Reihe nach: „die erste Komponente des dreigliedrigen Existenzialsatzes vom Typus Es war ein Mann“ (ebd., 221), die Füllung der obligatorischen Objektsstelle des Prädikats (ebd.) und die Füllung der

${ }^{19}$ d.h. bezüglich der Charakterisierung der Abnahme konkreter Dinglichkeit

${ }^{20}$ Möglich ist hier jedoch - neben der Kataphorik, gleichzeitig damit - auch eine anaphorische Bezugnahme, genau so, wie Admoni (ebd., 220) es auch sieht, vgl. hierzu näher 2.3.3. 
ersten Stelle im Elementarsatz (ebd., 222). Dies sind bereits syntaktische Anmerkungen. Möchte man ausgehend von obigen Beispielen Stufen annehmen und ein Stufenmodell aufstellen, d.h. gewissermaßen Ordnung in den Komplex bringen, zwischen dessen Elementen etliche Verbindungen möglich sind (ebd., 222), so setzt das Gradualität voraus, wobei betont werden muss, dass die einzelnen Stufen nicht immer scharf getrennt werden können, wie später zu zeigen sein wird. Zur ersten Orientierung sollen drei Belege angeführt werden:

(17) Als ich nun zu ihnen kam, und mich als Deserteur angab, nahmen sie mir das Gewehr ab, unterm Versprechen, mir's nachwerts schon wieder zuzustellen. (4) (B)

(18) Sophiechen ist confirmirt $u$. ich habe mein Theil dabei geweint, wie viel auch Deiner gedacht $u$. gefunden, daß wir keineswegs so gesinnt sind wie wir es sein sollten (114) $(\mathrm{SCH})$

(19) Es ist auch $\mathrm{Zu}$ verstehen, das mann auf solchen botten, welcher guth und drachtbar ist, keine weig oder natel holtzer, geren zieht, es müste dan seine besontere ursach haben (89) (J)

(17) repräsentiert, dass durch es eine Nominalphrase (NP) wiederaufgenommen wird, die ein neutrales Substantiv als Kern hat, das wiederum einen Gattungsnamen darstellt. In (18) bezieht sich es auf ein Adjektivprädikativ, das Teil der Prädikation oder des Prädikats im semantischen Sinn (IDS-Grammatik 1997: 601) ist, indem es zusammen mit der Kopula (sind) einen Prädikatsausdruck bildet (von Polenz 1985: 107, vgl. dazu weiter 2.2.2). (19) zeigt die Bezugnahme auf einen vorangehenden (Neben)Satz durch es. Hier wird ein kompletter Satzinhalt wiederaufgenommen. Vereinfacht ergeben sich folgende Stufen:

Semantik

1. Bezug auf einen Gegenstand

2. Bezug auf eine Prädikation

3. Bezug auf einen Satzinhalt syntaktische Kategorie

1. Bezug auf eine NP

2. Bezug auf eine Nicht-NP

3. Bezug auf einen Satz 
In den folgenden zwei Kapiteln gilt es dieses vereinfachte Schema auszuloten. Zunächst soll die semantische Beschreibung möglicher Bezugsobjekte erfolgen. Dabei werden drei Arten von Phorik durch es angenommen:

- implizite Phorik

- explizite Phorik

- syntaktisch gebundene Phorik (es-Korrelate)

Auf die ersten beiden Phorik-Arten wird in 2.2.1 und 2.2.2, auf die dritte jedoch erst in 2.3, nach der Darstellung der kategorialen Einführung von Inhalten, näher eingegangen, da diese in engem Zusammenhang mit festen kategorialen Realisierungen steht. Dies ist gleichzeitig der Grund dafür, dass sie überhaupt als eine eigene Art von Phorik durch es ausgewiesen wird.

\subsubsection{Semantische Bestimmung der es-Phorik: implizite Verweise}

Den Ausgangspunkt der Diskussion bilden der Beleg

(20) Sie muß fest von seine Treue u. Liebe überzeugt sein, denn es ist ein ernstes Verhältniß, sie corespondiren mit einander (13) (K)

sowie Askedals Beispiel

(21) Das Telefon klingelte. Ich hob den Hörer nicht ab. Es war Hanna.

Vergleicht man (20) und (21) mit (17)-(19) in 2.2, so fällt auf, dass keine Objekte im vorangehenden Textteil zu sehen sind, auf die explizit verwiesen wird. Trotzdem möchte ich im Folgenden dafür argumentieren, dass Phorik auch in (20) und (21) vorliegt. Es ist jedoch eine andere Art von Phorik als in den Belegen in 2.2. Ich nenne sie implizit und bringe sie weiter unten mit Implikaturen in Zusammenhang. Im Folgenden wird zunächst diese implizite Art von es-Phorik dargestellt. Im Anschluss daran wende ich mich der expliziten Phorik durch es zu. 
Askedal (1990: 214) führt sein Beispiel unter Typ 3 an, der anaphorische esVorkommen beinhaltet. Es ist allerdings ein Beleg,

wo ein noch nicht lexikalisch spezifizierter Aktant (semantischer Rolleninhaber) sich durch semantisch-pragmatische Implikation aus einer im vorhergehenden Kotext eingeführten Situation ergibt [Hervorhebung von mir, D.C.] und zunächst - möglichst "neutral" - durch es bezeichnet wird. Beispielsweise ist in (8) [die Originalnummer in Askedals Text, D.C.] von der Situation des Telefonierens die Rede, zu der im pragmatischen Normalfall ein(e) Urheber(in) des Telefonats gehört. (ebd.) ${ }^{21}$

\begin{abstract}
Ähnliche Beispiele mit dem gleichen, im obigen Zitat kursivierten, Kommentar finden wir bei ihm auf Seite 216, diese werden allerdings einem anderen, nämlich Typ 5 (,anaphorischer propositionaler bzw. Textbezug“) zugeordnet. Des Weiteren begegnet man bei Askedal (ebd.) Beispielen, die ein es mit ,allgemeinem narrativen Bezug“ (ebd.) enthalten. Es sind Bezugnahmen auf Satzinhalte, die aber nicht mit konkreten Konstituentenfolgen identifiziert werden können. Hinzu kommen es-Vorkommen mit Kontextbezug (1990: 218, Typ 9). Auch hier ist kein textuelles Bezugselement zu entdecken, der im Kotext beschriebene Kontext kann jedoch als Bezugsobjekt fungieren. Die Hinweise auf Phorik ohne konkreten Bezugsausdruck im Kotext $(1990: 214,216)$ und auf die problematische Zuordnung solcher Belege (ebd., 216, 218) zu einzelnen Typen führen zu der Frage, wie Verweise dieser Art behandelt werden können. Sind das überhaupt Verweise im klassisch-phorischen Sinne? In der Spezialliteratur zu es finden sie praktisch keine Beachtung. Unbekannt sind sie jedoch auch nicht. Behaghel (1923: 276f.) spricht bspw. von unmittelbarer vs. mittelbarer Anaphora:
\end{abstract}

Der durch das Prononomen aufgenommene Begriff kann in der Nachbarschaft unmittelbar ausgedrückt werden (unmittelbare Anaphora) [...] In manchen Fällen ist der bei der Aufnahme vorschwebende Begriff selber in der Nachbarschaft nicht ausgedrückt, er schwebt aber unausgesprochen vor der Seele, weil andere tatsächlich ausgesprochene Wörter mit ihm in irgendwelcher Verbindung stehen (mittelbare Anaphora).

${ }^{21}$ Askedal (1990: 213) unterscheidet zwischen Ko- und Kontext. Während Letzterer das außersprachliche Umfeld bezeichnet, steht Ersterer für das im vorangehenden Textteil tatsächlich Präsente. 
Harweg (1979: 192f., 195ff.) beschreibt mittelbare Anaphern als „TextKontiguitätssubstitutionen“. Die neuere Fachliteratur zu Anaphern verwendet vor allem die beiden Termini direkt vs. indirekt, s. Schwarz (1997) und (2000), SchwarzFriesel/Consten/Marx (2004) und Consten/Schwarz-Friesel (2007). Die Definition indirekter Anaphern ähnelt der mittelbarer Anaphern in hohem Maße: „Es gibt sprachliche Sequenzen, die anaphorische Ausdrücke aufweisen, obgleich kein direkter, also explizit genannter Antezedent im Text benutzt wird.“ (Schwarz 1997: 449, vgl. auch SchwarzFriesel/Consten/Marx 2004: 81 und Consten/Schwarz-Friesel 2007: 284). Auch der letzte Teil der Behaghel'schen Definition ist in neueren Arbeiten zu erkennen, indem gesagt wird, dass indirekte Anaphern ,in systematischen Relationen zu Einheiten der vorherigen Textstruktur“ stehen (Schwarz 2000: 98). Diese Einheiten werden Anker genannt (Consten/Schwarz-Friesel 2007). ${ }^{22}$ Wichtig ist, dass diese Anker nicht gleichzusetzen sind mit den direkten Antezedenten, aber sie sind „entscheidend für die Interpretation der indirekten Anapher“" (Schwarz 1997: 449). Es lässt sich also sagen, dass bei indirekten Anaphern der Ausdruck, auf den Bezug genommen wird, sich aus dem Gesagten ergibt. Die beiden wichtigsten Funktionen indirekter Anaphern sehen Consten/Schwarz-Friesel (2007: 284) in der Neufokussierung und der Neueinführung von Referenten. Allerdings gilt das hauptsächlich für definite NPs mit substantivischem Kern, die im Mittelpunkt der Analysen von Consten und Schwarz-Friesel stehen. ${ }^{23}$ Beide Hauptfunktionen werfen Fragen auf. So rührt Neufokussierung an der problematischen Abgrenzung der Phorik von Textdeixis. Dieses Problem wurde in 2.1 bereits angedeutet und muss hier erneut aufgegriffen werden. Die Neueinführung von Referenten ist grundsätzlich ausgeschlossen, da Pronomina semantisch zu dieser Leistung nicht geeignet sind. Hinzu kommt, dass (20)-(21) m.E. nicht gleich zu behandeln sind und bezogen auf es Differenzierungen vorgenommen werden müssen. Aus diesen Gründen ist eine einfache Anwendung der Theorie zu indirekten Anaphern auf es nicht möglich. Folgende Fragen müssen geklärt werden:

1. Was heißt es bezogen auf es-Vorkommen, dass Phorik nicht direkt vorliegt, sondern sich aus dem Prätext ergibt? Damit im Zusammenhang:

2. Was genau ist unter ,irgendwelcher Verbindung“ $\mathrm{zu}$ anderen, tatsächlich ausgesprochenen bzw. niedergeschriebenen Wörtern bzw. unter „systematischen

\footnotetext{
${ }^{22}$ Cornish (2005: 204) verwendet den Begriff ,,antecedent-trigger“.

${ }^{23}$ Sie (ebd., 286) weisen auf die Möglichkeit indirekter pronominaler Anaphern am Rande hin.
} 
Relationen“ zu verstehen, was für Anker kommen also im Allgemeinen und speziell bei es-Anaphern in Frage?

3. Wie trennt man indirekte Phorik und Textdeixis voneinander?

Frage 1 bezieht sich im Wesentlichen darauf, ob und wie der Terminus indirekte Anapher auf es bezogen werden kann. Dazu wird auf den Begriff der Implikatur zurückgegriffen. Frage 2 thematisiert Typen indirekter Verweise.

Der Begriff der Implikatur bei Grice (1975) ist darauf zurückzuführen, dass ein Kontrast besteht zwischen Gesagtem und tatsächlich Gemeintem, wenn Äußerungen gemacht werden. So bringt er (1975: 43) das Beispiel: „Suppose that A and B are talking about a mutual friend, $\mathrm{C}$, who is now working in a bank. A asks $\mathrm{B}$ how $\mathrm{C}$ is getting on in his job, and B replies, Oh quite well, I think; he likes his colleagues, and he hasn't been to prison yet $^{\text {“ }}$ und fügt hinzu: „I think it is clear that whatever B implied, suggested, meant, etc., in this example, is distinct from what B said, which was simply that $\mathrm{C}$ had not been to prison yet.“ Das Gemeinte erschließt sich einerseits durch die wörtliche Bedeutung des geäußerten Satzes (Kemmerling 1991: 323), andererseits basiert es auf pragmatischen Annahmen, bspw. darauf, dass der Sprecher mit dem Adressaten kooperiert. ${ }^{24}$ Dieses und andere pragmatische Prinzipien hat Grice (1975) unter den Begriff der „konversationalen Implikatur“ subsumiert. Für meine Zwecke einschlägig ist allerdings die andere Art von Implikaturen, nämlich die konventionale, bei der wörtliche Bedeutungen eine Rolle spielen: „In some cases the conventional meaning of the words used will determine what is implicated, besides helping to determine what is said.“ (ebd., 164). Implikaturen dieser Art „sind die Bestandteile des Äußerungsinhaltes, die sich zwar aus der wörtlichen Bedeutung des geäußerten Satzes ergeben, die aber nicht zum Gesagten zu rechnen sind“, so Kemmerling (1991: 325). Einen für die Analyse meiner Beispiele sehr nützlichen Rahmen bietet m.E. die Satzsemantik von Peter von Polenz (1985), da er den Unterschied zwischen konventional und konversationell durch die beiden Begriffe Mitbedeutetes und Mitgemeintes (1985: 302) anschaulich zum Ausdruck bringt. Das Mitbedeutete rührt vom Sprachwissen, es ist also derjenige Teil des Äußerunginhaltes, der vom Hörer oder Leser auf Grund seines Sprachwissens verstanden werden muss (ebd., vgl. auch Kemmerling 1991: 325). Da für meine Korpusbelege und deren Interpretation mitbedeutete Inhalte eine Rolle

\footnotetext{
${ }^{24}$ S. hierzu weiterhin von Polenz (1985: 299).
} 
spielen, wird im Folgenden unter Implikatur/Impliziertes dieses Mitbedeutete verstanden. ${ }^{25}$ Auch terminologisch soll im Weiteren der Begriff der Implikatur umgesetzt werden, indem bei Verweisen auf Impliziertes von impliziter, bei solchen auf sprachlich explizit Vorliegendes von expliziter Phorik gesprochen wird.

Was mögliche Typen angeht (Frage 2), so führen Consten/Schwarz-Friesel (2007: 285) - bezogen auf und veranschaulicht an nominalen Beispielen - meronymische (auf TeilGanzes-Beziehungen beruhende), verbsemantisch basierte und schemabasierte indirekte (in der vorliegenden Arbeit: implizite) Anaphern an:

Nicole stand vor der Tür zur Wohnung ihres Freundes, da bemerkte sie, dass das Schloss klemmte. (Meronymie zwischen der Tür und das Schloss)

Nicole wollte die Wohnung ihres Freundes aufschließen, da bemerkte sie, dass sie den Schlüssel verloren hatte. (verbsemantisch basiert: Schlüssel trägt die Instrument-Rolle von aufschließen)

Nicole wollte in die Wohnung ihres Freundes, da bemerkte sie, dass sie den Schlüssel verloren hatte. (schemabasiert: wollte in die Wohnung ihres Freundes ist zu allgemein, erst durch Schlüssel wird klar, dass sie aufschließen und nicht etwa klingeln wollte.)

In Schwarz (2000) ist die Darstellung möglicher Typen komplexer. Im fünften Kapitel ihrer Monografie analysiert sie die so genannte Verankerung, d.h. die Relation zwischen Ankern und den dazu gehörenden impliziten Anaphern, und setzt (2000: 118) lexikon-, weltwissenund inferenzbasierte Grundtypen impliziter Phorik an. Alle drei enthalten Subtypen, die im Grunde genommen den Typen in Consten/Schwarz-Friesel (2007) entsprechen und die ihrerseits weitere Untergruppen enthalten. Schwarz (2000: 118) weist darauf hin, dass die Klassifizierung nicht zu eindeutig abgrenzbaren Typen führt, sondern eher als Kontinuum zu betrachten sei. In der Tat lassen sich manche Beispiele nur schwer voneinander trennen, u.a. weil konzeptuelles Wissen auch bei lexikonbasierter Verankerung eingeschaltet werden kann (2000: 101f.). ${ }^{26}$ Nicht eindeutig genug ist überhaupt die Etablierung von

\footnotetext{
${ }^{25}$ Auch von Polenz (1985: 303) verwendet hierfür den Begriff Impliziertes.

${ }^{26}$ Man vergleiche etwa die Beispiele (25) auf Seite 103 und (76) auf Seite 111. Warum wird etwa bei (25) angenommen, dass Insassen in diesem Beispiel zum Rollenbereich des Fliegens gehören, während in (76) Krankenschwester durch Krankenhauszimmer im vorangehenden Satz nicht notwendig impliziert und dagegen das mentale Schema Krankenhaus aktiviert werden muss? Was ist denn der Unterschied zwischen dem Rollenspektrum eines Verbs und Schemata, die darüber hinausgehen sollen?
} 
inferenzbasierten Typen (ebd., 114), genauer gesagt ihre Trennung von weltwissenbasierten Typen, da bei beiden konzeptuelles Wissen aktiviert werde. Noch schwieriger ist es hier, zwischen semantischen Rollenzuweisungen und mentalen Schemata zu differenzieren. Neben diesen allgemeinen Problemen mit der Typologie von Schwarz (2000) darf nicht außer Acht gelassen werden, dass bezogen auf es auch deshalb mit Schwierigkeiten zu rechnen ist, weil Pronomina nur eingeschränkt, „unter bestimmten Voraussetzungen“ (ebd., 123, 127, vgl. auch Fußnote 23) als implizite Anaphern in Frage kommen. Was diese Voraussetzungen genau sind, davon erfährt man hier freilich wenig Konkretes. ${ }^{27}$ Schwarz (ebd., 129) formuliert zusammenfassend:

\begin{abstract}
Insgesamt bleibt aber zu betonen, daß die Verwendung von Pronomina als indirekte Anaphern keineswegs die gleiche Systematik aufweist, die bei definiten NPs zu beobachten ist, sondern vielmehr auf bestimmte Kontexte und Situationen (indirekte Pronominalanaphern finden sich weitaus häufiger im mündlichen Diskurs als in der Schriftsprache) beschränkt bleibt.
\end{abstract}

Die Ausführungen in Schwarz (2000: 127ff.) legen jedoch nahe, dass der Unterschied zwischen nominalen und pronominalen impliziten Anaphern darin liegt, dass bei Letzteren so komplexe mentale Verarbeitungsprozesse wie bei Ersteren nicht möglich sind, denn „Pronomina setzen generell die leichte und unmittelbare Erreichbarkeit ihrer Referenten, also explizite Repräsentationseinheiten in Text und Diskurs voraus [...]“ (ebd., 123) Dies mag stimmen, es ist aber keine Antwort auf die Frage, warum denn die den nominalen sehr ähnlichen Beispiele funktionieren. Man vergleiche etwa das Verhältnis zwischen Werkstatt und Sie in

Gestern war ich in der Werkstatt, um mich nach meinem Fernseher zu erkundigen. Sie haben gesagt, es würde noch drei Tage dauern. (ebd., 128)

mit dem zwischen Krankenhauszimmer und Krankenschwester in Beleg (76) auf Seite 111 (s. hierzu Fußnote 26). Noch interessanter ist hier das es, das auf die nicht erwähnte Reparatur verweist (ebd., 128). Diese ergibt sich wiederum offensichtlich aus dem

\footnotetext{
${ }^{27}$ Dafür werden jedoch (ebd., 123ff.) Sonderfälle präsentiert, die keine implizite Phorik darstellen, und es wird mit Hilfe zahlreicher Beispiele zu so genannten „anaphorischen Inseln“ dargelegt, dass Referenten grundsätzlich explizit durch eine NP realisiert werden müssen, um pronominal wiederaufgenommen werden zu können (ebd., 124f.).
} 
Werkstatt-Schema, insofern ist dieser Beleg im Schwarz'schen System ein Beispiel für schemabasierte implizite Anaphern. Weiterhin werden Beispiele folgender Art angeführt:

Hannes ist glücklich verheiratet, hörte ich gestern. Sie soll sehr lieb und nett sein. (ebd.)

In diesem Fall impliziert der adjektivische Prädikatsausdruck ist verheiratet den weiblichen Ehepartner, für deren Aufnahme das Pronomen sie gewählt wird. Auch Cornish (2005: 212f.) führt zwei ähnliche Beispiele an:

Joan is six months' pregnant and she has already knitted a bonnet and gloves for $i t$.

John got married last week. She's Swedish, if you want to know.

Im ersten Satz impliziert der Prädikatsausdruck is pregnant ein Baby als impliziten Antezedenten, auf den durch it Bezug genommen wird; im zweiten wird durch got married die Braut mitbedeutet, die als Bezugsausdruck für she gilt. In meinem Beleg

(22) Zu dem End kauft' ich im Eggberg, wo meine Dulcinee daheim war, etwas Salpetererde, und zugleich ihres Vaters Gaden - ihr zu lieb viel zu theuer; denn es war fast verloren Geld (10) (B)

der dem mit aufschließen und Schlüssel ähnelt, impliziert der Ankerausdruck kauft' ausgegebenes Geld, das die Instrument-Rolle bei kaufen trägt, als impliziter Bezugsusdruck fungiert und von dem am Ende gesagt wird, dass es (fast) verlorenes Geld ist. Der Unterschied zwischen diesen beiden Beispielen und den mit ist verheiratet und got married ist nach Cornish (2005: 214), dass die impliziten Antezedenten bei Letzteren so genannte „nuclear arguments“ (ebd.) darstellen, d.h. dem Prädikatsausdruck näher stehen (bei Verben: verbnäher sind) als etwa die Instrumental-Rollen bei kaufen und aufschließen. ${ }^{28}$ Der Hinweis auf die Bedeutung der Art von semantischen Rollen bei Cornish ist relevant, denn er nimmt (ebd., 213) an, dass implizierte Antezedenten nuclear, d.h. zentrale Argumente sein müssen, wenn implizite Phorik durch unbetonte, schwache Pronomina hergestellt werden soll. Denn nur zentrale Argumente sind „highly activated“ (ebd., 211) und dies ist

${ }^{28}$ Diese wären dementsprechend ,peripheral“. Solche Entscheidungen sind allerdings nicht unumstritten bzw. es müsste immer beim jeweiligen Verb konkret bestimmt werden, ob ein zentrales oder ein peripheres Argument anzunehmen ist. 
die Voraussetzung dafür, dass sie durch Pronomina „mühelos und unzweideutig“ (Schwarz 2000: 123) erreicht werden. Bei der Annahme zentraler und peripherischer Argumente greift Cornish (ebd.) auf Überlegungen $\mathrm{zu}$ der Hierarchie semantischer Rollen bei Prädikatsausdrücken (vor allem Verben) in angelsächsischen funktionalen Grammatiken zurück. Ähnliche Gedanken finden sich in der germanistischen Forschung etwa bei Welke (2002), der sich mit Rollenhierarchien auseinandersetzt und zu folgendem Schluss kommt: „Die Hierarchie semantischer Rollen resultiert aus der Hierarchie der syntaktischen Relationen [...]“ (2002: 99). Diese wiederum ist „ein syntaktischer Ausdruck der Perspektiviertheit des Verbs“ (ebd., 105), die bei jedem Verb abstrakte Argumentpositionen (Position 1, 2, 3 usw.) und dadurch auch eine Reihenfolge dieser Argumente festlegt (ebd., 107). Perspektiviertheit bedeutet also „eine bestimmte subjektiv (aber nicht individuell) motivierte Reihenfolge der Argumente eines Prädikats, d.h. deren unterschiedliche Auszeichnung [...]“ (ebd., 92). Dabei gilt immer das Argument als erstes, von dem aus das jeweilige Verb einen Sachverhalt perspektiviert. Solche Reihenfolgen sind natürlich immer dann interessant, wenn zu einem Verb mehrere Argumente gehören. Bezieht man die Gedanken Welkes auf das Kriterium der Zentralität bei Cornish, so lässt sich das jeweils erste Argument sicherlich als zentral einstufen. Was mit zweiten und dritten Argumenten hingegen gemacht wird, können wir hieraus nicht herleiten. ${ }^{29}$ Hinzu kommt, dass die Beispiele mit kaufen und Werkstatt für diese Analyse ein Problem darstellen, da Instrumental-Rollen nicht zentral sind und Werkstatt, weil kein Prädikatsausdruck, über gar keine Rollen verfügt. Trotzdem ist es als implizite Anapher in beiden Fällen möglich, wie die Daten dies zeigen, vgl. auch (23) unten. Der Widerspruch entsteht meiner Meinung nach einerseits deshalb, weil die meisten Beispiele auf maskuline, feminine und pluralische Pronominalformen Bezug nehmen. Das es ist aber bekanntlich „eine seltsame Ausnahme“ (Starke 1996: 405) unter den Personalpronomina, das Operationen wie Fokussierung, Koordinierung oder Modifizierung (etwa durch Partikeln) nicht zulässt (ebd., 406, Beispiele dort). Andererseits wird seine Fähigkeit, auf komplexe Inhalte, bspw. auf Propositionen oder Reihen von Propositionen, zu verweisen, alleine schon dadurch außer Acht gelassen, dass es unter den Beispielen kaum auftaucht.

\footnotetext{
${ }^{29}$ Überhaupt sind die von Welke (2002: 114f.) dargestellten Lexikoneinträge zu Verben problematisch, wenn man zunächst das erste Argument eines Verbs festlegen möchte. Auf Seite 152 liest man - bezogen auf das Tagalog, eine philippinische Sprache -, dass Verben entweder aktiv oder stativ sind, woraus die Perspektiviertheit hinsichtlich des ersten Arguments resultiert, was wiederum heißt, dass aktive Verben vom Agens aus perspektiviert werden. Kurz: Ob etwa das Agens das erste Argument ist, entscheidet man, indem man überprüft, ob das jeweilige Verb aktiv ist, was man wiederum so entscheiden kann, dass man sagt, aktive Verben werden vom Agens aus perspektiviert.
} 
Wie (20) und das Beispiel mit is pregnant zeigen, sind es nicht immer Verben, durch die ein impliziter Antezdens-Ausdruck entsteht. In (20) wird durch die beiden Substantive Treue und Liebe eine Beziehung zwischen zwei Menschen mitbedeutet, auf die implizit Bezug genommen wird und von der dann durch eine Charakterisierungskonstruktion behauptet wird, dass es sich dabei um ein ernstes Verhältnis handelt. Man könnte hier einwenden, dass eine implizite anaphorische Relation zwischen Verhältni $\beta$ und Treue + Liebe besteht. Dies ist zwar richtig, bedeutet aber nicht, dass durch es keine implizite Anaphorik hergestellt wird. Die Eigentümlichkeit dieser doppelt realisierten Anaphorik resultiert aus der Funktion solcher es-Kopulasätze. Für diese ist gerade typisch, dass sie den Antezedenten durch es zunächst neutral einführen und die genaue Identifizierung oder Charakterisierung desselben erst durch die NP erfolgt (Askedal 1990: 214). Zwischen (20) und den angeführten Beispielen für verbsemantisch basierte implizite Anaphern besteht eine weitere wichtige Differenz: Während die mitbedeuteten Antezedenten bei den genannten Verben mitbedeutete semantische Rollen darstellen, hat man es bei (20) mit Wortfeldern zu tun, indem Treue und Liebe dem gleichen Wortfeld wie Verhältniß zugeordnet werden können. Mit Peter von Polenz (1985:309) sind hier „die sog. semantischen Relationen zwischen Wörtern/Lexemen, die für die systematische Darstellung von Wortfeldern im Wortschatz einer Sprache unerläßlich sind“, gemeint. Zusammenfassend möchte ich die Phorik in den oben kommentierten verbalen und substantivischen bzw. adjektivischen Beispielen wortsemantisch basierte implizite Phorik nennen. Trotz wichtiger Differenzen (Aktivierung von Verbargumenten - s. kaufen-Geld in (22) - vs. Wortfelder, s. (20)) lässt sich hier implizite Phorik auf die Semantik einzelner Wörter zurückführen.

Eine nächste Stufe stellen das Beispiel mit Werkstatt oben, (21) und der Beleg

(23) Der Mörder ward ertapt, gefangen genomen, nach Marpurg uffs Schloß geführt. Was nun es vor ein Außgang wird gewinen, das wird die Zeit geben. (32) (BL)

dar. In all diesen Belegen geht es nach meiner Ansicht darum, dass erst das Nacheinander bzw. die Verbindung einzelner Wörter und Ausdrücke zum Implizierten führt. Hier gelten also Verbindungen verschiedener Einheiten als Anker und implizieren den Antezedenten, der durch es wiederaufgenommen wird. Bspw. implizieren ertapt, gefangen genomen und uffs Schloß geführt durch ihr Aneinanderreihen offenbar einen gerichtlichen Prozess gegen den Mörder. Das es verweist auf diesen Prozess. Genau auf diese Art von 
Wortverbindungen soll im Weiteren der Begriff Schema (Consten/Schwarz-Friesel 2007: 285) angewendet werden. In diesem Sinne stellt die Verbindung von ertapt, gefangen genomen und uffs Schloß geführ das Prozess-Schema her. In Askedals Beispiel (21) lässt sich auf Grund der Bedeutung von Das Telefon klingelte. der/die Telefonierende insofern ermitteln, als das Klingeln des Telefons automatisch bedeutet, dass jemand anruft, also ein Agens (der semantische Rolleninhaber, wie Askedal formuliert) mitbedeutet wird. Auf dieses Agens nimmt dann es in Es war Hanna. Bezug. Hie wird das Schema des Telefonierens aktiviert. Den Ankerausdruck für die zu realisierende Bezugnahme bilden nicht Telefon und klingeln alleine, sondern ihre Verbindung. Im Harweg'schen System (1979: 195f.) wäre dies ein Beispiel für logisch begründete Kontiguitäts-Substitutionen, bei dem zum Actum (jemand telefoniert, was sich aus Das Telefon klingelte. ergibt) ein Agens gehört. Wichtig ist, dass erst bei Realisierung des ganzen es-Satzes klar wird, dass zu es ein impliziter Antezedent gehört, der Agens ist. Möglich wäre nämlich u.a. auch folgende Variante:

Das Telefon klingelte. Es hat mich gestört, also ging ich nicht ' $r a n$.

Hier bezieht sich es auf Das Telefon klingelte. Es ist also erst der es-haltige Kopulasatz mit dem Eigennamen Hanna, der die für (21) gültige Interpretation ermöglicht. ${ }^{30} \mathrm{Im}$ Falle des Beispiels bei Monika Schwarz (2000: 128) ist es m.E. nicht Werkstatt alleine, das als Anker der impliziten Phorik dient. Der Finalsatz mit Fernseher muss angeschlossen werden, denn der gewünschte implizierte Antezedent kann nur so hergestellt werden, vgl. die etwas abgeänderte Form dieses Beispiels:

Gestern war ich in der Werkstatt, um die neuen Reifen, die ich bestellt hatte, abzuholen. Sie haben gesagt, es würde noch drei Tage dauern.

Hier bezieht sich es auf gar keinen Fall auf irgendeine Reparatur. Die Rede von der Bestellung der Reifen ergibt eine andere Interpretation, nämlich dass die Lieferung der neuen Reifen im Sinne von „bis sie da sind“ oder „,bis Sie sie abholen können“ drei Tage dauern soll. Das es verweist dann auf diesen Vorgang des Dauerns.

\footnotetext{
${ }^{30}$ Vgl. auch Klaus Fischers Beispiel Die Tür ging auf. Es war Paula. und seine Anmerkung dazu (2009: 179): „The es [...] is anaphoric. It refers back to previous text, although the precise nature of the link, that is, that it refers to the implied causer of the event expressed in the first sentence, only becomes clear with the predicative at the end of the second sentence $[\ldots]^{\text {“ }}$
} 
Den bisher behandelten Beispielen ist gemeinsam, dass sie semantisch motiviert sind. Dabei kann allerdings der Weg zum Antezedenten unterschiedlich sein. In bestimmten Fällen gilt die Semantik einzelner Wörter als Grundlage der Interpretation impliziter Phorik, in anderen Fällen hat man es mit Schemata $\mathrm{zu}$ tun: Erst die Verbindung von unterschiedlichen Einheiten führt zum Implizierten, das dann als Bezugsgröße für es fungiert. Entsprechend dieser Differenz möchte ich implizite Phorik derart weiter differenzieren, dass von wortsemantisch basierter einerseits und von schemabasierter andererseits gesprochen wird. Wie die Beispiele in Schwarz (2000) zeigen, ist bei schemabasierter impliziter Phorik damit zu rechnen, dass ggf. pragmatisches Wissen eingeschaltet wird.

Bei von Polenz (1985: 309) werden auch grammatikalische Implikaturen erwähnt. Er weist auf das Passiv und den Komparativ hin, die jeweils ihre Konversen mitbedeuten. ${ }^{31}$ Behaghel (1923: 278f.) bringt unter Typ A (mittelbare Anapher, ebd., 278) folgendes Beispiel:

ich will sehen, daß ich ihn wieder finde - er hat's (mich wiedergefunden)

„Die Nachbarschaft enthält eine andere Form desselben Verbal-Paradigmas“, so Behaghel (ebd.). Dies ist gewiss ein anderer Typ impliziter Phorik als die wortsemantisch basierte, es beruht nämlich auf einem Wortparadigma, das morphologisch spezifizierte Wortformen desselben Wortes umfasst. In diesem Fall substituiert es zwar das verbale Lexem wiederfinden (samt seinem Akkusativobjekt), aber eben nicht in dessen Originalform, d.h. Präs.1.Ps.Sg., sondern in einer zu demselben Paradigma gehörenden, die dem Verbalkomplex, d.h. der Präsensperfekt-Form des Satzes mit der Anapher, angepasst wird. Ein weiteres Beispiel ist:

(24) Sie zogen fur den Berg, doch heimlicherweise, und wolten in einnemen, aber es wolt innen nicht glücken. (12) (BL)

\footnotetext{
${ }^{31}$ Bei Passivsätzen wären diese Konversen die entsprechenden Aktivsätze. Im Falle des Komparativs handelt es sich darum, dass etwa die Formel $x$ ist größer als $y$ die Konverse $y$ ist kleiner als $x$ impliziert. (ebd.) Das Mitbedeutete ist also sozusagen in der syntaktischen Konstruktion automatisch mit kodiert.
} 
Der Verbalkomplex (kursiv) beinhaltet in (24) auch eine modalisierende Komponente, nämlich wolten, ${ }^{32}$ anaphorisch wiederaufgenommen wird jedoch „,nur noch“ in einnemen, bzw. hieße es nach seiner Anpassung an wolt innen nicht glücken, wenn man präzis sein möchte, in einzunemen. Auch diese Form ist auf Grund des gegebenen Verbparadigmas zu erschließen.

An dieser Stelle sind erste Antworten auf die oben gestellten Fragen 1 und 2 möglich:

Antwort 1: Dass Phorik sich aus dem Prätext ergibt, indem kein expliziter Antezedent-Ausdruck vorliegt, bedeutet, dass der Verweis über einen Anker erfolgt, der sozusagen als Vermittler zwischen Substituendum und Substituens eingeschaltet wird. Das Substituendum wird dabei durch den Anker impliziert.

Antwort 2: Es werden zunächst folgende Typen impliziter Phorik durch es angenommen:

- wortsemantisch basierte implizite Phorik

- schemabasierte implizite Phorik

- grammatisch motivierte implizite Phorik

Es wird an dieser Stelle erneut darauf aufmerksam gemacht, dass, wie oben gezeigt, die Modifizierung der Schwarz'schen Typologie aus den Eigenschaften des es und der Analyse der angeführten Belegen resultiert. Dies führt einerseits zu Differenzierungen, andererseits aber auch $\mathrm{zu}$ Vereinfachungen. Bspw. sind nominalsemantische paradigmatische Beziehungen, wie etwa Meronymie (Schwarz 2000: 104), zwischen implizitem Antezedenten und es nicht möglich, hier kommen nur definite NPs mit substantivischem Kern in Frage.

Schwierigkeiten ergeben sich bei beiden Frage-Antwort-Paaren. Bei Frage (und Antwort) 1 sind sie theoretischer Natur und knüpfen an Frage 3 an, auf die nun eingegangen werden soll. Hierbei geht es, wie oben bereits kurz angedeutet, um die Frage, wie Phorik

${ }^{32} \mathrm{Zu}$ dem Begriff der Modalisierung, wie er an dieser Stelle verstanden wird, und zu Modalverben als grammatischen Mitteln der Modalisierung siehe Eisenberg (2006: 93). 
und Deixis auseinander gehalten werden können. Die Fragestellung ist berechtigt, da in 2.1 behauptet wurde, dass

Deixis der Neuorientierung bzw. der Fokussierung auf neu eingeführte Elemente dient, während

Phorik zwecks des Rückbezugs auf Bekanntes und der Beibehaltung der Orientierung eingesetzt wird.

Behauptet wurde des Weiteren in Anlehnung auf die Fachliteratur zu impliziten (dort: indirekten) Anaphern, dass ihre Funktion in der Einführung neuer Textreferenten besteht. Offensichtlich sind Neueinführung und Neuorientierung miteinander eng verwandt. Außerdem wird in der IDS-Grammatik Sie in dem Beispiel

Der Fürst heiratete wieder. Sie war eine Schauspielerin.

als Deixis interpretiert, weil hier ,auf Gegenstände erst orientiert wird und die Ausdrücke im Diskurs zu akzentuieren wären.“(1997: 547). Wohlgemerkt, analoge Beispiele mit got married oder heiratete/verheiratet bei Cornish (2005) bzw. Schwarz (2000) werden als Fälle impliziter Phorik analysiert. Eine ausführlichere Arbeitsdefinition impliziter Anaphern von Schwarz (2000: 49) lautet zudem:

Bei den indirekten Anaphern handelt es sich um definite Ausdrücke, die in interpretativer Abhängigkeit $\mathrm{zu}$ bestimmten Ausdrücken der vorausgehenden Textstruktur stehen und zwei textreferentielle Funktionen haben: die Einführung neuer (bisher noch nicht explizit erwähnter) Textreferenten und die Weiterführung des globalen referentiellen Bezugs.

Wie man sieht, scheinen implizite Anaphern zentrale Merkmale der beiden Zeigmodi, d.h. phorische und deiktische Funktionen, zu vereinen, vgl. hierzu auch Cornish (2005: 202). Ausgehend von der Beispieldiskussion und der Definition für implizite Anaphern ist grundsätzlich zu fragen, ob Impliziertes als phorischer Bezugspunkt fungieren kann. Die Antwort hängt m.E. davon ab, welcher Bestandteil der Definition als primär erachtet wird. Nimmt man etwa die Beispiele mit heiraten, so kann man dort sagen, dass durch das 
Pronomen Elemente eingeführt werden, die im vorangehenden Kotext in Form von Wörtern oder sonstiger Ausdrücke nicht präsent sind, folglich als neu angesehen werden müssen. Akzeptiert man allerdings das Konzept der Implikaturen, so kann man nicht behaupten, dass das Implizierte in dem Sinne neu ist wie neu eingeführte und fokussierte Einheiten in klassischen Deixis-Beispielen. Eine mögliche Lösung bietet meiner Meinung der Vorschlag Diewalds. Sie weist (1991: 115) auf die Problematik hin:

Die zum Teil ungelöste Frage, wann etwas als vorerwähnt, indirekt vorerwähnt bzw. als semantisch oder pragmatisch impliziert gelten soll, erschwert dabei zusätzlich die Entscheidung, welcher von beiden Zeigmodi im Einzelfall vorliegt.

Entscheidend ist nach Diewald (ebd., 122ff.), dass man in den einzelnen Fällen den dominanten Zeigmodus identifiziert. Hier kann man gleich einwenden, dass sich dabei Probleme ergeben können, weil Textverständnis nicht unbedingt homogen ist und einzelne Leser zu verschiedenen Textinterpretationen neigen dürften. Es kann also dazu kommen, dass nur noch individuelle Entscheidungen zu der Annahme von Phorik oder zu der von Deixis führen. Im Falle des es glaube ich jedoch, dass in den diskutierten Beispielen die einzig mögliche Funktion nur die phorische sein kann. Dafür spricht, dass es schwach, unbetont ist und deshalb auch nicht fokussieren kann. Dies wird untermauert durch den Kontrast mit das und seinen femininen wie maskulinen Pendants. Da es im Paradigma der Personalpronomina eine Sonderstellung einnimmt, indem es einerseits für maskuline und feminine Personalpronomina nicht typischen syntaktischen Restriktionen unterliegt (Starke 1996: 405f.), andererseits aber auch über besondere phorische und syntaktische Potenzen verfügt (Admoni 1976, Askedal 1990), folgt aus der Entscheidung für Phorik gegenüber Deixis nicht, dass etwa sie und er in obigen Gebrauchsweisen ebenfalls phorisch sind. Ganz im Gegenteil: Die Annahme von Deixis kann berechtigt sein. Er und sie (und auch die pluralischen Formen) sind betonbar und können fokussieren. Folgt man weiterhin der Argumentation in Diewald (1991: 115, 122), so ist eine a priori scharfe Trennung beider Zeigmodi gar nicht vonnöten, sondern erst in konkreten Texten vorzunehmen.

Bei Frage (und Antwort) 2 resultieren Schwierigkeiten aus der Empirie und führen zu Problemen bei der Abgrenzung einzelner Typen voneinander bzw. bei der Trennung impliziter von expliziter Phorik. Zunächst kann man der Frage nachgehen, wie mit 
Wortbildung umgegangen werden kann. Behaghel (1923: 278) führt Wortbildungen, ganz genau Ableitungen, als Fälle mittelbarer Phorik an, vgl. folgende zwei Beispiele:

dahero ist er auch sträflich, aber solche soll er von euch zu seiner Zeit erhalten

diese Hesliche scheint zu schertzen, aber gedulde nur, er soll dir schon vergehen

Im ersten Beispielsatz steht solche für das deadjektivische Derivatum Strafe, das durch sträflich impliziert wird. Der zweite Beleg zeigt, dass das deverbale Derivatum Scherz impliziert und durch er darauf Bezug genommen wird. Ein analoger Beleg aus meinem Korpus ist

(25) wenn ich ernsthaft krank wäre würde ich nach Salzburg fahren, dort weiß ich doch 2 empfohlene Ärzte- Aber es ist nichts ernsthaftes nur ein bisl hergenommen hat mich das alles, ich liege halt viel herum. (1154) (LB)

Hier kann Krankheit als Implikat des Adjektivs krank und so als impliziter Antezedent zu es angesehen werden. Dies gilt trotz der Inkongruenz in Genus, inkongruente Bezüge durch es sind nämlich bekannt und in meinem Korpus reichlich anzutreffen. Dass ableitungsbasierte implizite Phorik nicht den gleichen Status haben kann wie die oben als grammatisch gekennzeichnete, sieht man daran, dass im Falle der verbalen Paradigmen (wiederfinden, einnehmen) nur es möglich ist, was aber bei Ableitungen nicht der Fall ist, wie dies Behaghels Beispielen zu entnehmen ist. Außerdem ist die Ableitung eines Wortes aus einem anderen, was eine Wortbildungsart darstellt, nicht gleichzusetzen mit dem Verhältnis zwischen Wortformen innerhalb eines Paradigmas. Aus diesem Grund werden Belege mit Ableitungen zum wortsemantisch basierten Typ impliziter Phorik gerechnet.

Besonders problematisch ist die Situation bei dem oben als grammatisch motivierte implizite Phorik bezeichneten Phänomen. Es muss nämlich grundsätzlich anders funktionieren als implizite Phorik in den Belegen (20)-(23) und in den der einschlägigen Fachliteratur entnommenen Beispielen. Denn bei grammatisch gesteuerter impliziter Phorik hat man es mit einem Wortparadigma zu tun, dessen Mitglied als Ankerausdruck (vgl. wieder finde oben) eine andere Wortform (wiedergefunden) desselben Paradigmas implizieren soll, die als Antezedent-Ausdruck fungiert. Überhaupt kann man fragen, was es 
denn heißt, dass grammatisch impliziert wird, wenn von Phorik die Rede ist. von Polenz (1985: 309) verbindet die von ihm kurz erwähnten grammatischen Implikatur-Fälle (PassivAktiv und Komparativ) auch nicht mit phorischen Merkmalen. Behaghels Beispiel ist auch deshalb problematisch für meine Systematik, weil seiner Logik folgend auch manche Beispiele von Askedal (1990: 215) und ein Beleg aus meinem Korpus angeführt werden könnten:

aber ich zog sie nicht auf mit ihrer Vergeßlichkeit und Flüchtigkeit, wie ich es sonst wohl tue.

Ich zögerte, ob ich mir ihre Kontokarte suchen lassen sollte. Aber dann ließ ich es.

(26) Ich erschrak und lauschte durchs schlüßelloch. und sahe, das sein bedienter | welcher ein sehr verschmitzer kerll war | vor Ihm auf den knien lag und Ihn mit aufgehobnen händen baht $\mid$ von müller abzulaßen | es wäre sonst sein unglük. (3) (DM)

Bei diesen Belegen spricht Askedal (ebd.) von Bezugsgrößen unterschiedlichen Umfangs, wobei er keinerlei Konnex zwischen diesen und seinen kurzen Ausführungen zu Implikaturen (ebd., 214) herstellt. Es sind Verweise auf Propositionen und Textabschnitte (ebd., vgl. hierzu weiter unten). Für ihn sind es also „ganz normale“ phorische Gebrauchsweisen von es. Die Kursivierungen oben zeigen deutlich, worin das Problem besteht: Versucht man, wie Askedal (ebd., 215f.), die tatsächlichen Bezugsgrößen auszuformulieren, so führt es zu Ergebnissen wie „,ich ziehe sie mit ihrer Vergeßlichkeit und Flüchtigkeit auf“ beim ersten, „mir ihre Kontokarte suchen zu lassen“ beim zweiten Beleg und etwa „wenn er von müller nicht abläßt“ in (26). Im ersten Fall werden Tempus und Negation „abgezogen“, im zweiten wird eine Infinitivkonstruktion, ohne Modalität (sollte) und Subjekt (ich), produziert und in (26) ein wenn-Satz mit Finitum aufgebaut. Ganz zu schweigen von den Junktoren, die in den ersten beiden Beispielen auch „verschwinden“, wenn die präzise Form der Bezugsgrößen erschlossen werden soll. ${ }^{33}$ Wenn Askedal paraphrasiert, so verfolgt er damit wohl das Ziel zu zeigen, wie Bezugseinheiten der eshaltigen Struktur angepasst werden, um etwa zu sehen, wie die jeweilige Bezugsgröße formal realisiert würde, wenn er an der Stelle von es stünde. Überhaupt ist dabei m.E. der Status von Paraphrasen obiger Art zu klären: Spiegeln sie reale Änderungen in der Phorik wider oder sind es nur formulierungstechnische Hilfsmittel des Linguisten? Ich komme auf

\footnotetext{
${ }^{33}$ Weitere Beispiele für „Abzüge“ findet man in Askedal (1990: 215f.) sowie in Cho (1975: 24ff.)
} 
diese Frage weiter unten zurück. Führt man nun Askedals Beispiele mit dem von Behaghel zusammen, so kann man meiner Meinung nach von Anpassung der Form der Bezugsgröße an den anapherhaltigen Satz, nicht aber von implizierten Bezugsgrößen sprechen. Der Unterschied zwischen den zwei Beispielgruppen liegt darin, dass auf der einen Seite (Behaghel) rein grammatische Anpassungen vorgenommen werden, indem die entsprechende Wortform aus demselben Wortparadigma gewählt wird, während auf der anderen Seite (Askedal) - parallel zu eventuellen wortformenbezogenen Modifikationen bei der phorischen Wiederaufnahme so genannte Diktumserweiterungen (IDS-Grammatik 1997: 602) entfallen. Ich glaube also, bei dieser Art von Phorik wird nicht impliziert, sondern es findet eine grammatische und/oder semantische Anpassung der Bezugsgröße an den anapherhaltigen Satz statt. Und dies ist folglich nicht gleichzusetzen mit dem Prozess, bei dem etwa aus krank Krankheit entsteht, Geld durch kaufen oder Reparatur durch Werkstatt impliziert wird. Letztere Fälle sind keine Anpassungen im obigen Sinne. Diesem Gedankengang folgend wird die oben eingeführte Typologie derart modifiziert, dass der dort als grammatisch eingestufte Typ zur expliziten Phorik gezählt wird. Entsprechend bleiben als Typen impliziter Phorik durch es

- wortsemantisch basierte implizite Phorik, vgl.:

(25) wenn ich ernsthaft krank wäre würde ich nach Salzburg fahren, dort weiß ich doch 2 empfohlene Ärzte- Aber es ist nichts ernsthaftes nur ein bisl hergenommen hat mich das alles, ich liege halt viel herum. (1154) (LB)

- schemabasierte implizite Phorik, vgl.:

(23) Der Mörder ward ertapt, gefangen genomen, nach Marpurg uffs Schloß geführt. Was nun es vor ein Außgang wird gewinen, das wird die Zeit geben. (32) (BL) ${ }^{34}$

\subsubsection{Semantische Bestimmung der es-Phorik: explizite Verweise}

Nach den Ausführungen zu impliziten Verweisen wird es im Folgenden darum gehen, explizite Phorik, die zweite Art von Phorik, darzustellen. Im Mittelpunkt der Analysen stand bisher, was implizite Phorik heißt, wie sie entsteht und welche Typen mit Bezug auf es

\footnotetext{
${ }^{34}$ In diese Einteilung lassen sich auch Askedals Beispiele (10) bzw. (21)-(23) (1990: 215f.) einfügen, die alle Belege für wortsemantisch oder schemabasierte implizite Phorik sind.
} 
etabliert werden können. Nicht ausgeführt wurde dabei, was für Inhalte als implizierte Bezugsgrößen in Frage kommen. Wenn wir uns die Beispiele in Erinnerung rufen, so lässt sich sagen, dass auf Gegenstände oder Personen - vgl. etwa (21) - und auch komplexere Propositionen (Werkstatt-Beispiel) verwiesen werden kann. Dieser Gesichtspunkt soll nun näher betrachtet werden, indem die möglichen Bezugsgrößen ermittelt werden. Dabei wird davon ausgegangen, dass diese auch im Bereich der impliziten Phorik gelten. Als Diskussionsgrundlage dienen folgende Belege:

(27) Sie zogen fur den Berg, doch heimlicherweise, und wolten in einnemen, aber es wolt innen nicht glücken. Es war ein uberauß ungestümige Nacht von Regen und Wind, von Brausen und Sausen, als in langer Zeitt nicht gewesen ist. (12) (BL)

(28) Die Statt ward gantz und zumal außgeplundert, die arme Burgersleuth musten alle mit letigen Händen davon, konden nicht behalten, was sie am Leibe hatten. Ach Gott, der ellende Zeit. Der Oberste Königsmargk bleib uff Omeneburg ligen die sex Wochen, so lang das Läger wehret. Es war der schwedische Jenneral genant Frangel und war Jenneral Bönighaußen. Es waren die Hessen der selbige Jenneral genant Johan Gisso. [S. 59] Es war ein sehr grosses Volk, das sichs zu verwundern war, und war eben im Somer von S. Johainstag bis nach Jacobi. Ja, es war noch nicht gnung. (17) (BL)

(29) Gerade von meiner Verheurathung an wollt' ich mit nichts geringerm beginnen, als - der Welt völlig abzusagen, und das Fleisch mit allen seinen Gelüsten zu kreutzigen. Aber o ich einfältiger Mensch! Was es da für ein Gewirre und für Widersprüche in meinem Innwendigen absetzte. (17) (B)

Es ist schwierig, die Phorizität von es in (27) genau zu bestimmen (daher auch keine Kursivierung). Man könnte hier die Bezugnahme etwa so paraphrasieren: „Als das alles passiert ist, hatten wir 'ein uberauß ungestümige Nacht von Regen und Wind, von Brausen und Sausen, als in langer Zeitt nicht gewesen ist."“ In diesem Sinne nimmt es das Vorangehende wieder auf und steht neutral für die raumzeitliche Situation, in der sich die genannten Ereignisse (Man zieht auf den Berg, will die Burg einnehmen, es klappt aber nicht.) abspielen. Diese Situation wird charakterisiert, indem ein Kopulasatz mit Nacht gewählt wird, der auch erweitert wird. Dieser Fall grenzt an implizite Phorik. Askedal (1990: 215) formuliert, dass die Situation „implikationell gegeben“ ist. Ich glaube jedoch, 
dass hier nicht von Implikation und folglich auch nicht von impliziter Phorik im Sinne der obigen Überlegungen die Rede sein kann, da Handlungen, Geschehen oder Vorgänge immer zeitlich und örtlich situiert sind und diese Situiertheit demzufolge automatisch vorliegt. Daher betrachte ich Situiertheit zwar als gegeben, aber eben nicht als implikationell gegeben.

Für (28) soll hier „rückweisendes verallgemeinernd-summierendes“ es gewählt werden (Admoni 1976: 219). Dieses es nimmt

den Bedeutungsgehalt eines Abschnitts des vorhergehenden Texts auf. Der Abschnitt kann von ganz verschiedener Beschaffenheit und Länge sein. Selbst eine ganze zusammenhängende Erzählung kann der Erzähler (Verfasser) z. B. mit dem Satz beschließen: Ja, so war es. (ebd.)

Bei Summierung ist eine Textpassage als Ganzes zu verstehen, nicht die einfache Addierung einzelner Sätze. Technisch gesprochen: Wenn man den Bezugsausdruck markieren möchte, so müsste man auch alle Relationen zwischen den Sätzen markieren, so u.a. Junktionen verschiedenster Art. Auch Schwarz-Friesel/Consten/Max (2004: 76) weisen auf diese Möglichkeit hin: „Des Weiteren können Ereignisse, die raumzeitlich, kausal oder thematisch zusammenhängen, mittels Anapher summiert werden und sind dann nur noch als einheitlicher Diskursreferent repräsentiert“. Asher (1993: 234f.) spricht im Falle solcher Bezugnahmen von „sum of events“ und „event summation“:

Like individuals, events may sum together [...] Events that are temporally, spatially, causally, or thematically connected may sum together to form larger events [...] Natural language metaphysics treats sums of events, however, as individual, singular events not aggregates or groups thereof. Thus [...] a singular pronoun like this, that or it may anaphorially pick up a sum of event.

Wie im Englischen besteht auch im Deutschen die Möglichkeit, durch die neutrale Form es auf mehrere abstrakte Objekte (s. dazu weiter unten) bzw. auf deren Summe anaphorisch Bezug zu nehmen.

(29) steht für Fälle, in denen durch es auf Propositionen Bezug genommen wird. Eine saubere Trennung von Belegen dieser Art vom verallgemeinernd-summierenden esGebrauch ist zugegebenermaßen nicht möglich, denn es ist schwierig, eine klare Trennlinie 
zwischen mehreren Propositionen und zusammenhängendem Ganzen von Propositionen zu ziehen. Auch Askedal (1990: 215) führt beide Belegtypen zusammen an (bei ihm Typ 5). Er (ebd.) spricht aber gleichzeitig von ,propositionale[m] bzw. Textbezug“ und schreibt, dass der Umfang der Objekte, auf die verwiesen wird, sehr unterschiedlich sein kann. Trotz dieses Kategorisierungsproblems bin ich der Meinung, dass an konkreten Textstellen Differenzierungen möglich sind, wie (28) und (29) dies zeigen. Bezüge auf Propositionen bedürfen aber auch deshalb einer näheren Betrachtung, weil sie eine sehr heterogene Gruppe darstellen und gewöhnlich Bezügen folgender Art gegenübergestellt werden:

(30) Es wollte mir aber kein Essen zu Leibe. (Es kame mir aber hernach zustatten, als die Not größer ward.) (23) (MD)

In (30) handelt es sich um einen es-Verweis auf einen Gegenstand. Diese Art von Gegenüberstellung propositionalen und gegenstandsbezogenen Verweises ist in der Fachliteratur bekannt (Schmidt 1987, Askedal 1990, Zifonun 2001), sie wird auch in dieser Arbeit als zentral erachtet. Auf gegenstandsbezogene Phorik wird nach der Darstellung propositionaler Bezüge eingegangen. Dieser wiederum soll an dieser Stelle eine kurze Zusammenfassung der bisherigen Ergebnisse vorgeschaltet werden. Um terminologische Klarheit zu schaffen, wird im Folgenden von Arten und Typen der es-Phorik gesprochen. Wie oben schon gezeigt wurde, werden drei Arten der Phorik angenommen. Innerhalb dieser drei Arten (kursiv) sollen folgende, bisher behandelte oder noch zu thematisierende Typen ihren Platz finden:

implizite Phorik

- wortsemantisch basiert

- $\quad$ schemabasiert

explizite Phorik
- $\quad$ Bezug auf eine Situation
- $\quad$ summierender Bezug 


\section{- $\quad$ Bezug auf Propositionen \\ - $\quad$ Bezug auf Gegenstände}

syntaktisch gebundene Phorik

In der Fachliteratur wird der Unterscheidung zwischen Verweisen auf Propositionen und solchen auf Gegenstände auch terminologisch Rechnung getragen. Zifonun (2001: 71, 122) spricht in Anlehnung an Asher (1993) bei Ersteren von einer Bezugnahme auf abstrakte Objekte, Schwarz-Friesel/Consten/Max (2004) und Consten/Schwarz-Friesel (2007) verwenden den Terminus Komplexanapher. ${ }^{35}$ Dabei sind die Begriffsbestimmungen sehr ähnlich. So liest man bei Zifonun (2001: 71):

Nicht nur auf Entitäten, die durch NP eingeführt wurden, kann phorisch Bezug genommen werden, sondern auch auf solche, die durch Sätze, Infinitivkonstruktionen oder Prädikatsausdrücke konstituiert wurden. Dabei sind, wie die sprachlichen Kontextbedingungen bezeugen, eine ganze Reihe unterschiedlicher Denotatstypen zu unterscheiden, etwa Sachverhalte, Ereignisse bzw. „Eventualitäten“ (mit weiterer Subklassifikation) und Tatsachen sowie Eigenschaften.

Consten/Schwarz-Friesel (2007: 275) führen aus, dass Komplexanaphern Anaphern sind, „deren Antezedent eine propositionale Struktur (d.h. ein Satz, eine VP oder ein satzwertiger Infinitiv) ist und die sich dementsprechend auf komplexe Referenten wie Ereignisse, Prozesse oder Zustände beziehen.“ Propositionen können als eine der beiden Hauptkomponenten von Dikta gefasst werden (IDS-Grammatik 1997: 599), ${ }^{36}$ sie stellen Sachverhaltsentwürfe dar. Dikta wiederum werden (ebd., 597) definiert als „die Bedeutung einer kommunikativen Minimaleinheit, das, was mit ihr - in einer spezifischen Interpretation - gesagt werden kann. ${ }^{\text {‘37 }}$ Im Folgenden schließe ich mich der Begrifflichkeit in der IDS-Grammatik (1997) und der Argumentation in Zifonun (2001) an, was darauf zurückgeführt werden kann, dass Schwarz-Friesel/Consten/Max (2004) und

\footnotetext{
${ }^{35}$ Auch Schmidt (1987: 105) nimmt eine ähnliche Differenzierung vor, er trennt pro-propositionale von prophrasaler Phorik. Dem folge ich in diesem Kapitel nicht, weil formale und semantische Merkmale entsprechend der methodischen Festlegung in diesem Kapitel - zunächst nicht zusammen behandelt werden sollen.

${ }^{36}$ Die andere Hauptkomponente ist der Modus dicendi, also das Wie des Sagens.

${ }^{37}$ Zum Begriff der kommunikativen Minimaleinheit vgl. IDS-Grammatik (1997, Kapitel B3).
} 
Consten/Schwarz-Friesel (2007) hauptsächlich nominale Anaphern untersuchen, ${ }^{38}$ während sich die Überlegungen Zifonuns konkret auf das es beziehen. In ihren Ausführungen ist zu lesen, dass abstrakte Objekte hinsichtlich Denotatstypen weiter unterteilt werden können, etwa in Sachverhalte, Ereignisse, Tatsachen oder Eigenschaften, und sie bringt (ebd., 72) Beispiele für alle diese Optionen. Vgl. hierzu auch folgende drei Belege:

Ereignis:

(31) Was ist eigentlich passiert, wenn man diese Angepaßtheit durchbrochen hat? Ist es nicht verstanden worden, ist es reglementiert worden? (16) (SB)

Tatsache:

(32) Der einte war, daß ich noch viel zu wenig Holz hatte, ungeachtet Mstr. Brunner mir gesagt, es sey genug, und es erst itzt einsah, als er an die oberste oder Firstkammer kam. (12) (B)

Eigenschaft:

(33) Sophiechen ist confirmirt $u$. ich habe mein Theil dabei geweint, wie viel auch Deiner gedacht u. gefunden, daß wir keineswegs so gesinnt sind wie wir es sein sollten (114) $(\mathrm{SCH})$

Der Denotatstyp Eigenschaft wird von allen anderen getrennt, weil er gegenüber Tatsachen und Ereignissen „ungesättigt“ sei (ebd.). Man kann sowohl für als auch gegen dieses Vorgehen argumentieren. Im letzteren Fall kann man Bezugnahmen, bei denen das wiederaufzunehmende Element die Prädikativfunktion erfüllt, im Zusammenhang mit Prädikaten so behandeln wie die anderen Denotatstypen oben. Bevor diesbezüglich eine Festlegung getroffen wird, soll kurz auf Prädikate und ihren Status in Propositionen eingeganden werden, um im Anschluss daran den „Prädikativ-Faden“ wieder aufzunehmen.

\footnotetext{
${ }^{38}$ Obwohl in Schwarz-Friesel/Consten/Max (2004: 70, 75) ein Beleg für es als Komplexanapher und mehrere für englisches $i t$ in der gleichen Funktion angeführt werden, wird in Consten/Schwarz-Friesel (2007: 275f., 287) für die „Nichtverwendbarkeit von Personalpronomina“ als Komplexanapher argumentiert und in Fußnote 10 (ebd., 276) sogar behauptet, dass laut einer Korpusstudie es als Komplexanapher in 0\% der Fälle vorkomme. Dieser Widerspruch lässt sich teilweise auflösen, wenn man berücksichtigt, dass die in Frage stehenden Fälle die Verwendung von $d$-Pronomina (konkret: dies und das) zeigen. Diese jedoch folgen sowohl in syntaktischer wie auch in textpragmatischer Hinsicht teilweise anderen Regularitäten als es: $d$-Pronomina können im Gegensatz zu es in der Funktion des direkten Objekts die Anfangsposition im Satz besetzen und werden oft anadeiktisch gebraucht, wie Consten/Schwarz-Friesel darauf ja selber hinweisen (ebd.).
} 
Dazu bedarf es zunächst der genauen Bestimmung von Propositionen. Die IDS-Grammatik (1997: 601, 679) gibt folgende Definitionen:

Propositionen ergeben sich - in elementarer Form - durch das Zusammenwirken zweier komplementär angelegter Komponenten: eines Prädikats und - abhängig von diesem eines oder mehrerer Argumente. Das Prädikat leistet dabei eine Charakterisierung, die Argumente entwerfen die Gegenstände, denen diese zugedacht wird.

Prädikate kommen so ins Spiel: Sprachliche Sachverhaltsentwürfe werden - anders als graphische Entwürfe - als Charakterisierungen realisiert. Charakterisierungen bestehen in der Anwendung eines Charakteristikums auf etwas, das damit charakterisiert wird. Für das Zustandekommen eines Sachverhaltsentwurfs sind mithin zwei Komponenten unabdingbar: ein Charakteristikum [Prädikat, D.C.] und ein Gegenstand der Charakterisierung [Argument, D.C].

Beide Definitionen suggerieren auf den ersten Blick Gleichrangigkeit der Komponenten. Implizit ist jedoch auf die zentrale Rolle des Prädikates zu schließen, indem einerseits in einem Einschub in der ersten Definition (1997: 601) „abhängig von diesem“ zu lesen ist, andererseits in den Darlegungen zum Verbalkomplex behauptet wird (ebd., 1239), dass dieser als typische Realisationsform des Prädikats das Orientierungszentrum der Proposition bildet. Dies deutet darauf hin, dass Prädikaten und den zu ihnen gehörenden Ausdrucksformen eine besondere Aufgabe zukommt. ${ }^{39}$ Für die Darstellung in dieser Arbeit bedeutet das wiederum, dass Bezüge auf Prädikate - zwar vereinfachend, jedoch aus ihrer zentralen Stellung abgeleitet - so behandelt werden wie solche auf (ganze) Propositionen. In diese Reihe könnten nun auch Bezugnahmen auf Eigenschaften eingefügt werden, indem Ausdrücke in prädikativer Funktion als Teile des Prädikats angesehen werden (ebd., 1107). ${ }^{40}$ Sie prädizieren in Verbindung mit (Kopula)Verben, ,wirken also semantisch gesehen wie einstellige Verben.“ (ebd., 1106) Der formale gemeinsame Nenner sind dann bei all diesen Bezügen verbale Einheiten. Diese Argumentation ist jedoch diskutierbar: Es ist ein wesentlicher Unterschied, dass der Bezug auf Eigenschaften in einer besonderen syntaktischen Konstellation, nämlich als Prädikativ + Kopulaverb, realisiert wird und das

\footnotetext{
${ }^{39}$ In der Tat werden verbale Wortgruppen, insbesondere solche mit einem Verbalkomplex, herausgestellt und von Phrasen jeglicher Art abgegrenzt (ebd., 83f.), vgl. hierzu weiter unten.

${ }^{40}$ Dies gilt für so genannte Identitätsaussagen des Typs Sie ist seine Mutter nicht; diese sind jedoch Sonderfälle, denn Prädikative weisen i.d.R. Gegenstände als Elemente von Klassen aus (ebd., 1106).
} 
es dabei nicht als selbstständiges Argument $\mathrm{zu}$ deuten ist. Aus diesem Grund ist es gerechtfertigt, Bezüge auf Eigenschaften in Prädikativfunktion herauszustellen und sowohl von Bezügen auf Propositionen - da kopulahaltig - als auch von solchen auf Gegenstände da wegen des Kopulaverbs doch verbal - zu trennen. Interessant sind in dieser Hinsicht Belege mit Partizip-II-Formen, die das Bild differenzieren:

Aber das erkannt' ich wohl, und war davon überzeugt, und bin es noch in der gegenwärtigen Stunde, daß sie für meine Umstände, unter allen die ich bekommen hätte, weit weit die tauglichste war (14) (B)

Bei Beispielen dieser Art stellt sich die bekannte Frage nach dem wortkategorialen Status des Partizip II (Adjektiv oder Verb?), die wiederum eng verbunden ist mit der Diskussion Kopula + Adjektiv vs. Zustandspassiv mit Passivhilfsverb, vgl. dazu Eisenberg (2006: 132f.) bzw. Maienborn (2007) und Welke (2007). Deutet man überzeugt als Adjektiv, so rückt dies die Analyse der Bezugnahme in die Nähe zum Eigenschaftspol. Schreibt man hingegen dem Partizip II einen verbalen Status zu, so landet man in der Nähe des verbalen, d.h. propositionalen, Pols. Offensichtlich muss hier auch die Lexikalisierung von Partizipien zu Adjektiven, was wohl oft vorliegt (vgl. etwa etwas ist mit Staub bedeckt), mit berücksichtigt werden, was wiederum zur Folge hat, dass ein skalar geordneter Bereich von Adjektiven und verbalen Partizipien anzusetzen ist. Dies rechtfertigt m.E. auch den Zwischenstatus von Bezügen auf Eigenschaften in prädikativer Funktion.

Nach der Diskussion über Prädikative stellt sich noch die Frage, wie mit Beispielen umgegangen werden kann, in denen auf Propositionen sozusagen nicht in ihrer Original-, sondern in abgeänderter Form Bezug genommen wird. Ich möchte in diesem Zusammenhang die kurz angedeutete Diskussion zum Status der Paraphrasen von Askedal (1990: 215f.) wieder aufgreifen. Zur Erinnerung sei ein Beleg angeführt:

aber ich zog sie nicht auf mit ihrer Vergeßlichkeit und Flüchtigkeit, wie ich es sonst wohl tue. (ebd.)

Mit Askedal kann man sagen, dass in diesem Beispiel die Proposition abzüglich Negation und Tempus (genauer: unter Änderung des Tempus) wiederaufgenommen wird. Die Anpassung in Tempus betrifft eine Verbalkategorie (Präteritum zu Präsens), die Auflösung der Negation aber nicht. Wohlgemerkt, beide Änderungen werden durch die syntaktisch- 
semantische Konstellation des Folgesatzes (wie ich es sonst wohl tue) ausgelöst. Die Negation gehört nicht zur Grundausstattung des Prädikats, sie ist eine Komponente der Proposition, die nicht obligatorisch mit dazu gehört, wenn man eine Elementarproposition aus Prädikat und Argument(en) zusammengesetzt bekommen möchte (IDS-Grammatik 1997: 601). Wenn diese über ihre beiden Hauptbestandteile hinausgehend spezifiziert werden, so handelt es sich dabei häufig um so genannte „geltungsrelevante Diktumserweiterungen“ (ebd., 602). Diese umfassen u.a. auch die erwähnte Negation und Modalfunktionen, aber auch Propositionsspezifikationen bezüglich „Ort, Zeit und sonstiger[r] situationelle[r] Gegebenheiten“ (ebd., 601, Beispiele dort). Fallen bei Paraphrasen solche Erweiterungen weg, so bleiben immer noch gültige (Elementar)Propositionen bestehen. Es muss dabei auch darauf aufmerksam gemacht werden, dass diese Art von Anpassung getrennt werden muss von der eher grammatischen, nämlich von der bezüglich Verbalkategorisierungen wie Tempus und Modus. Denn bei Verbalkategorisierungen betreffenden Änderungen entfällt nichts, somit führen diese auch nicht zum „Schwinden“ von Propositionen. Problematischer ist meiner Meinung nach die Beurteilung von Änderungen, die etwa das Subjekt betreffen. In allen Beispielen Askedals bleibt es gleich: Es ist in der Bezugsgröße bzw. der Einheit mit es immer dasselbe Subjekt. Man kann sich aber auch ein Beispiel wie folgt vorstellen:

(34) Ich möchte Fisch essen. Péter will es aber nicht.

In (34) steht es nicht für Ich möchte Fisch essen, auch nicht für Ich esse Fisch, sondern für das Kursivierte. Aus Ich „wird“ Peter. Auch das Modalverb „entfällt“. Es wird durch es nur Fisch essen wiederaufgenommen, und zwar wohl deshalb, weil es in die grammatische Konstellation des Folgesatzes nur so passt. Ist es nun immer noch ein Propositionsbezug? Wohl nicht, wenn zu einer Proposition „unverzichtbar“ ein Prädikat und ein oder mehrere Argumente gehören (IDS-Grammatik 1997: 601). Im gegebenen Beispiel werden das Prädikat und ein Argument phorisch erfasst. Nicht aber das ganze Prädikat und das andere Argument, das als Subjekt erscheint. Man kann diesen Gedankengang auf einer allgemeineren Ebene weiterführen und fragen, wie etwa das Kursivierte im folgenden Beispiel aus Zifonun (2001: 72) zu paraphrasieren ist:

Gestern haben wir den Weihnachtsbaum geschmückt. Es hat drei Stunden gedauert. 
Was nimmt es hier genau auf? „den Weihnachtsbaum zu schmücken“ nach einer Paraphrase wie „Den Weihnachtsbaum zu schmücken hat drei Stunden gedauert“ oder „Gestern haben wir den Weihnachtsbaum geschmückt“ in einer Paraphrase wie „Dass wir gestern den Baum geschmückt haben, hat drei Stunden gedauert“? Es ist nicht egal, denn im ersten Fall fehlen das Subjekt und gestern und grammatisch ist es eine Infinitivkonstruktion mit $z u$. Im zweiten Fall ist der ganze Satz da. Paraphrasen führen also teilweise bestimmt zu willkürlichen Ergebnissen und folglich besteht auch die Gefahr, dass willkürlich kategorisiert wird, weil man so oder so paraphrasieren kann. Aus diesen Gründen wird hier festgehalten, dass es methodisch sinnvoll ist, von solchen Paraphrasen abzusehen und sich grundsätzlich darauf zu konzentrieren, ob bei der jeweiligen Bezugnahme das Prädikat und seine verbale Ausdrucksform (gänzlich oder teilweise) betroffen sind oder nicht. Sind sie es, so wird im Rahmen der vorliegenden Arbeit von pro-propositionaler Phorik gesprochen, auch wenn dies den in der IDS-Grammatik formulierten Anforderungen an Elementarpropositionen (1997: 601) nicht entspricht, weil Änderungen etwa bezüglich des Subjekts vorgenommen werden.

Von pro-propositionaler Phorik zu differenzieren sind Verweise auf Gegenstände, die hauptsächlich nominal realisiert werden. Dies entspricht auch der grundsätzlichen Differenzierung zwischen dem verbalen und dem nominalen Bereich in anderen grammatischen Beschreibungsbereichen. Bedeutend und häufig anzutreffen sind Bezüge auf Argumente als Propositionsteile, vgl.:

(35) Das Korn fängt an zu grünen und zu wachsen, es wird überall gepflügt mit Ochsen oder Pferde, gespannt vor dem Pflug. (10) (SCHG)

(36) «Schau, ich bring Dir ein kleines Mädchen, nimms mit in die Stube und diesen Mann, komme nach.> (7) (SCHG)

„Argumente sind, was Prädikate zu Propositionen vervollständigt“, heißt es in der IDSGrammatik (1997: 730) und es steht dort auch, dass diese Leistung erreicht wird, indem dabei Gegenstände entworfen, genannt oder fortgeführt werden bzw. auf sie verwiesen wird (ebd.). Am wichtigsten sind bei der es-Phorik Gegenstandsentwürfe als wiederaufzunehmende Größen. Diese wiederum werden (ebd.) analog zu Sachverhaltsentwürfen (d.h. Propositionen) gefasst: „Ein GEGENSTANDSENTWURF [...] 
spezifiziert die Bedingungen, die erfüllt sein müssen, damit etwas als der und der Gegenstand gelten kann.“ Zu betonen ist dabei einerseits, dass zwar bei Bezugnahmen auf Gegenstandsentwürfe diese häufig gleichzeitig auch Argumente sind, es jedoch nicht immer der Fall zu sein braucht:

(37) Da habe ich noch nichts unternommen, weil ich durch das schlechte Wetter und mancherlei verhindert war nach Maria Grün zu hole. Sollte es aber schön bleiben so habe ich es für morgen Nach- // mittag vor. (2442) (LB)

In diesem Beispiel ist das Wetter als Teil einer Präpositionalphrase in adverbialer Funktion nach den Bestimmungen in der IDS-Grammatik (1997: 733) kein Argument, aber sicherlich ein Gegenstandsentwurf. Andererseits kommen nicht nur Gegenstandsentwürfe als Bezugsgrößen in Frage, sondern auch Gegenstandsnennungen, vgl.:

(38) Den Bub will ich dann in Ailingen dem Lehrer Grossmann übergeben, es ist mir am Weg. (6) (SCHG)

Hier ist Ailingen weder Argument noch Gegenstandsentwurf. Es ist ein Eigenname. Trotzdem bleibt gültig, dass bei Bezügen auf Teile von Propositionen Gegenstandsentwürfe in Argumentposition die Mehrheit der Fälle ausmachen.

Ich fasse zusammen: Vereinfachend wird stellvertretend für Gegenstandsentwürfe und -nennungen Gegenstand gebraucht. Das Bild expliziter Phorik lässt sich wie folgt präzisieren: Grundsätzlich kann zwischen Bezügen auf Gegenstände und solchen auf NichtGegenstände unterschieden werden. Letztere werden in Anlehnung an Zifonun (2001) abstrakte Objekte genannt. Unter abstrakte Objekte sollen entsprechend der Gegenüberstellung Gegenstand vs. Nicht-Gegenstand auch Bezugnahmen auf Situationen und Eigenschaften bzw. summierende Verweise fallen. Es entsteht folgendes Schema:

\section{explizite Phorik}

Bezug auf abstrakte Objekte

- Bezug auf Situationen

- $\quad$ Summierender Bezug 
- $\quad$ Bezug auf Propositionen inkl. Bezug auf das Prädikat oder seine Teile

- Bezug auf Eigenschaften

\section{Bezug auf Gegenstände}

\subsubsection{Morphosyntaktische Bestimmung der es-Phorik}

Im Mittelpunkt dieses Kapitels steht die kategoriale Einführung der es-Bezugsobjekte. Am Ende von 2.2 wurde behauptet, dass auf Sätze, NPs und Nicht-NPs bezogen werden kann. Vereinfacht man diese Reihe weiter, so lässt sich sagen, dass Phrasen und Sätze die wichtigsten syntaktischen Strukturen darstellen, in denen Bezugsgrößen vorkommen. Daher gilt es im Folgenden, diese beiden Begriffe zu erläutern, soweit es für die Zwecke der vorliegenden Arbeit nötig ist. Die Beschränkung der Ausführungen auf ,soweit hier nötig“ ist wichtig, da eine exhaustive Darstellung aller vorhandenen Konzepte zu den Begriffen Phrase und Satz im Rahmen dieser Arbeit nicht angestrebt werden kann. Es wird das Ziel verfolgt, typische kategoriale Erscheinungsformen der in 2.2.2 dargelegten semantischen Einheiten herauszustellen. So wie in 2.2.1 und 2.2.2 der Weg vom Allgemeinen zum Konkreten geführt hat (implizite Phorik $\rightarrow$ Situationsbezug $\rightarrow$ Propositionsbezug $\rightarrow$ Gegenstandsbezug), so soll in diesem Kapitel analog dazu beim „Größeren“ angefangen werden, um dann zum „Kleineren“ zu kommen. Dabei stellt sich zunächst die Frage: Was steht ganz oben? Unter kategorialem Aspekt kann man diesbezüglich nur ausweichen und sagen, es gibt Bezugsobjekte, die kategorial nicht fassbar sind. Dies gilt für Verweise auf Situationen und für das verallgemeinernd-summierende es. So kann etwa die raumzeitliche Situation in dem Beleg

(39) also musten die Kayserischen weichen und musten inen das Schloß lassen, dann es war im Winter. (21) (BL)

nicht über morphsyntaktische Kategorien erreicht werden. Das Gleiche gilt für den Fall, wenn auf einen ganzen Textabschnitt oder das vorher Erwähnte summierend verwiesen wird, vgl.: 
(40) als ich zurückkam, schlüpfte ich davon in mein Zimmer und setzte mich hin und las Deine Briefe durch und da ward's als ob Du mit mir sprechen würdest so lieb, so gut. Wenn 's doch wieder einmal sein könnte! (1529) (LB)

Bei (40) könnte man höchstens darauf hinweisen, dass auf mehrere Sätze samt Relationen jeglicher Art zwischen ihnen Bezug genommen wird. Obwohl in solchen Fällen im morphosyntaktischen Bereich wenig Exaktes festgelegt werden kann, so ist dabei zu betonen, dass gerade bei dieser Art von Phorik das maskuline und feminine Pendant zu es (er und sie) nicht einsetzbar sind. Bei propositionalen Bezugnahmen ist zwar der Gebrauch von es bedeutend, aber vor allem bei Nominalisierungen sind auch die beiden anderen Personalpronomina anzutreffen, vgl. Zifonun (2001: 71, Fußnote 20). Sieht man von den situationellen und summierenden Verweisen ab, so bleiben Sätze und Phrasen als die wichtigsten Kategorien. Zuerst soll auf Sätze, im Anschluss daran auf Phrasen eingegangen werden.

In einem ersten Schritt ist es möglich, einen generalisierten Satzbegriff zu Grunde zu legen. Ein solcher ist das Konzept des Elementarsatzes von Wladimir Admoni (1990: 4f.):

Der Begriff des Elementarsatzes umfaßt alle syntaktischen Strukturen, die zu einem der logisch-grammatischen Satztypen gehören und die nach den Richtlinien erweitert werden können, die für den selbständigen Satz gelten. Als Elementarsatz tritt also jeder Satz auf, der in den wichtigsten Zügen mit der Struktur des selbständigen Satzes übereinstimmt, ohne Rücksicht darauf, ob er eine abgeschlossene Einheit darstellt, und unabhängig davon, welche Rolle er im Redestrom spielt. Selbständiger Satz, Hauptsatz, Nebensatz, beigeordneter Satz - alle diese Sätze sind Elementarsätze.

Meine Belege zeigen, dass durch es auf Elementarsätze Bezug genommen werden kann, vgl. etwa folgende Beispiele:

(41) «Je gelt! deine Anne ist auch verplempert; dein Vetter Michel war so glückselig, und sie hat schon ein Kind». - Das fuhr mir ja durch Mark und Bein; indessen ließ ich's den argen Unglückboten nicht merken (7) (B) 
(42) Gibt es heute immer noch ähnlich viele Wörter mit Konnotationen oder ist es vielleicht nicht mehr so notwendig, in der heutigen Sprache solche Wörter zu verwenden? (12) (SB)

(43) meine gnädige frau $\mid$ die Es gemerkt hate $\mid$ das müller alles aufboht $\mid$ hielt mich in der größten stränge (2) (DM)

(41) repräsentiert den Bezug auf einen kommunikativ selbständigen Satz, der hinsichtlich seiner Komplexität als einfach einzustufen ist. Das Kursivierte in (42) stellt eine Infinitivkonstruktion (IK) in Subjektfunktion dar. Es ist nicht selbständig. (43) beinhaltet einen Objektsatz als Bezugsgröße, ebenfalls unselbständig, eingeleitet durch den Subjunktor das. Diese drei Beispiele sind bezüglich ihrer Selbständigkeit, Funktion und Form unterschiedlich, können aber alle als Elementarsätze betrachtet werden. Da aber der Begriff Elementarsatz den Bestandteil Satz beinhaltet, muss erörtert werden, was in der vorliegenden Arbeit als Satz begriffen wird. Es bietet sich zunächst eine vorrangig formorientierte Bestimmung an, wie sie in der IDS-Grammatik (1997: 86f.) zu finden ist. Demnach liegt ein Satz vor, wenn die in Frage stehende Einheit a) ein finites Verb beinhaltet und b) die strukturell und kontextuell notwendigen Komplemente des Verbs realisiert sind. Dabei entsteht ein Konflikt vor allem bezüglich Infinitivkonstruktionen wie der (42). Laut der Definition in der IDS-Grammatik sind IKs keine Sätze, weil sie kein finites Verb enthalten und die notwendigen Komplemente zwar erscheinen, nicht aber das Subjekt, zumindest nicht direkt. IKs sind nämlich dafür bekannt, dass die Interpretation ihrer Subjektstelle entweder an einem Ausdruck in dem Obersatz erfolgt, der die IK enthält (= Orientierung), oder aber erst kontextuell oder wissensbezogen fixiert wird (ebd., 1385). Bspw. wird die Subjektstelle in (42) durch den Kontext derart erschlossen, dass es sich dabei wohl um die Menschen handelt, von denen im Text die Rede ist (im Sinne von „man“). Andererseits entsprechen IKs den Anforderungen an Elementarsätze, sie sind subordinierte Sätze. Um diesen Widerspruch zu beheben, werden IKs ,als eine Art verkürzter Satz aufgefasst [...], in dem die Subjektstelle nicht besetzt ist.“ (Eisenberg 2006: 64) Es sprechen auf der einen Seite syntaktische Parallelen zwischen IKs und dass-Sätzen dafür, dass sie für meine Zwecke gleich behandelt werden (ebd., 354): 
- Die Satzglieder in syndetischen Nebensätzen und IKs weisen die gleiche Reihenfolge auf;

- beide haben das Verb an ihrem Ende stehen und

- Objekte sind gleichermaßen austauschbar, für Adverbiale gelten dieselben Positionsregeln.

Weiterhin kann man syntaktisch argumentieren, dass das oberflächensyntaktische Fehlen des Subjekts insofern eine Besonderheit darstellt, als außer dem Subjekt alle anderen Komplemente des Valenzträgers realisiert sind. Diese Besonderheit ist wiederum auf den gesonderten Status des Subjekts unter den Komplementen zurückzuführen. Mit Eisenberg (ebd., 35) kann gesagt werden, dass es gegenüber den anderen, lexikalisch regierten Komplementen kategorial regiert wird, da i.d.R. gilt, dass jedes Verb ein Nominativkomplement nehmen kann (ebd., 177, vgl. hierzu auch Eroms 2000: 188ff.). Funktional kann als ein Argument für die Behandlung von IKs als subordinierte Elementarsätze angeführt werden, dass sie, so wie etwa dass-Sätze, als Komplemente auftreten können. Aus diesen Überlegungen werden IKs in dieser Arbeit als (Elementar)Sätze gezählt. ${ }^{41}$ Somit bleibt es dabei, dass die drei Beispiele (41)-(43) esBezüge auf Elementarsätze darstellen. Neben IKs muss darauf aufmerksam gemacht werden, dass häufig nicht auf einzelne Elementarsätze, sondern auf mehrere gleichzeitig verwiesen wird. Dabei kann es sich um miteinander koordinierte wie auch um subordinierte, also in einem Satzgefüge auftretende Elementarsätze handeln:

(44) Wie es noch weider wird abgehen und die liebe Frucht wird inbracht werden, das weiß der liebe, getreue Gott und mir werden es erfahren. (26) (BL)

(45) Endlich entschloß ich mich, selber eins zu bauen, und sagte es meiner Schönen. (11) (B)

In (44) werden zwei Objektsätze koordiniert, während es in (45) auf ein Satzgefüge Bezug nimmt. ${ }^{42}$ Einen Sonderfall sententialen Bezuges stellen kataphorische Verweise auf Elementarsätze im selben Satzgefüge dar, vgl.:

\footnotetext{
${ }^{41}$ Ich möchte betonen, dass aus der Darstellung hier nicht folgt, dass die Zuweisung eines Sonderstatus an IKs (vgl. IDS-Grammatik 1997: 84, auch Eisenberg 2006 widmet IKs ein eigenes Kapitel) in einem anderen Rahmen nicht gut begründet werden kann.

${ }^{42}$ Hier ist auch die Interpretation möglich, nach der ein Bezug zwischen es und dem subordinierten Elementarsatz hergestellt wird.
} 
(46) doch seit Du mich gebeten, ich solle mich schonen, halt ich's für meine Pflicht Alles zu vermeiden, was mir schaden kann. (99f.) (SCH)

Hier gelten besondere Konstellationen bezüglich Stellung und Setzung des es, der Bezugsgröße und der Richtung der Phorik, die daraus folgen, dass der Bezug innerhalb desselben Satzgefüges stattfindet, was wiederum eine grammatikalisierte Form des Bezuges auf Elementarsätze bedeutet. Diese Art von Phorik durch es, bekannt als Korrelat-es, stellt die dritte Art von Phorik in meinem System dar (syntaktisch gebundene Phorik, vgl. 2.2). Ihre Bearbeitung wird wegen der Komplexität des Themas und der Statusbestimmung des Korrelat-es in dieser Arbeit in Kapitel 2.3 und teilweise in das zum nicht-phorischen es ausgelagert.

Neben Fällen, bei denen auf mehrere Elementarsätze Bezug genommen wird, ist von Interesse, wenn Antezedenten in Form von Einheiten unterhalb der Satzebene in Erscheinung treten. Unter solchen Einheiten sind in erster Linie Verben zu verstehen, vgl.:

(47) u. doch fehlte mir die Demuth dazu; kurz, ich möchte verzweifeln, doch darf ich es ja nicht (115) (SCH)

(48) In dieser bangen Nacht desertirten viele; neben andern auch Bruder Bachmann. Für mich wollt' es sich noch nicht schicken, so wohl's mir sonst behagt hätte. (1) (B)

Wenn von verbzentrierten Wortgruppen die Rede ist, so unterscheidet die IDS-Grammatik (1997: 83) Verbalkomplexe und Verbgruppen. Verbalkomplexe sind Gruppen,

in denen die Verbform durch Kombination eines finiten Hilfs- oder Modalverbs mit einem - von ihm regierten - infiniten Vollverb (Infinitiv, Partizip II) gebildet wird; weitere infinite Verbformen können zwischengeschaltet sein (ebd.).

In (47) bezieht sich es auf den infiniten Teil des Verbalkomplexes, nämlich auf das Vollverb. In (48) ist eine Bezugnahme auf ein finites Vollverb zu sehen. Zwar findet sich in der IDS-Grammatik keine Definition zu Verbgruppen, aber auf Grund zweier Abbildungen (ebd., 83f.) ließen sich Verbgruppen herstellen, indem das finite Verb oder der jeweilige Verbkomplex um Komplemente und eventuell auch Supplemente erweitert wird. Durch die 
Bindung aller Komplemente einschließlich des Subjekts ans Verb entstünden am Ende ganze Sätze als Verbgruppen (ebd.). ${ }^{43}$ So hätte man als mögliche Verbgruppen in (48) In dieser bangen Nacht desertierten und In dieser bangen Nacht desertierten viele. Sowohl Verbalkomplexe wie auch Verbgruppen werden in der IDS-Grammatik (ebd.) nicht als Phrasen aufgefasst. Dies hat unterschiedliche Gründe, die einerseits die Stellungsregularitäten des Finitums (ebd.), andererseits die dem Verbalkomplex zukommenden speziellen Aufgaben betreffen (ebd., 1239f.). ${ }^{44}$ Phrasen sind Wortgruppen, „deren Elemente funktional zusammengehören, aufeinander folgen und in ihren Formeigenschaften von einem Element gesteuert werden können, z.B. das alte Haus, völlig verrückt, auf der Bank.“ (ebd., 69) Als wichtigstes Kriterium erscheint i.d.R. das Vorhandensein eines lexikalischen Kopfes, des zentralen Elementes der jeweiligen Phrase. Der lexikalische Kopf ist deshalb von besonderer Bedeutung, weil er vielfach Formmerkmale anderer Elemente in der Phrase bestimmt (ebd., 72). Verbalkomplexe sind jedoch auch hierarchisch geordnet, ihre Bestandteile gehören funktional zusammen. Ihr Vollverb wird in Eisenberg (2006: 52) als lexikalischer Kopf aufgefasst. Anders als die IDS-Grammatik (1997) geht Eisenberg (2006: 51ff.) jedoch von syntaktischen Köpfen und Kernen aus. Kerne entsprechen dabei weitgehend dem Konzept des lexikalischen Kopfes, Köpfe werden in Anlehnung an die Begriffsbestimmung morphologischer Köpfe (bspw.: -ung in Derivata) definiert. Teilweise führt diese Unterscheidung zu anderen Ergebnissen, etwa bei Präpositionalphrasen (PP) (Typ: in die Schule), in denen Eisenberg (ebd., 53) die Präposition in als Kopf, die Nominalphrase die Schule als komplexen Kern analysiert, während Präpositionen in der IDS-Grammatik als lexikalische Köpfe (d.h. als Kerne im Sinne Eisenbergs) von PPs bestimmt werden. Für die Argumentation an dieser Stelle ist von Interesse, dass verbale Gruppen sich von Phrasen nicht unterscheiden, indem sie hierarchisch aufgebaut werden und über einen lexikalischen Kopf (Kern) verfügen. Aus diesem Grund werden sie hier als Phrasen betrachtet (VP). Ihrer besonderen Rolle in der Konstitution von Sätzen wird semantisch insofern Rechnung getragen, als sie analog zu Sätzen als Vertreter pro-propositionalen Bezuges verbucht werden.

\footnotetext{
${ }^{43}$ Dieses Konzept erinnert in gewisser Hinsicht an das spezifischer Einheiten bei Jean Fourquet (1970: 36), die durch die sukzessive Anbindung verschiedener Glieder an das Verb (= Konnexion) entstehen.

${ }^{44}$ So steht das Finitum - gegenüber der Stellung der Teile anderer Phrasen - in Aussagesätzen i.d.R. getrennt vom infiniten Teil. Verbalkomplexe sind außerdem typische Vertreter von Prädikaten, das Organisationszentrum der Proposition, sie regeln Zeitbezüge und stellen modale Charakterisierungen her (ebd., 1239f.). Ich füge noch hinzu: Zeit- und modale Bezüge können zwar auch durch andere, nicht verbale Wortgruppen zum Ausdruck kommen, verbale Einheiten tun dies jedoch vielfach mittels grammatikalisierter Verbalkategorisierungen wie Tempus und Modus.
} 
Wohl die wichtigste Rolle bei der phrasalen es-Phorik spielen Nominalphrasen. Es sind Phrasen, deren Kopf ein Nomen ist (ebd., 74). In diesem Fall wird meistens darauf hingewiesen, dass es und die Bezugs-NP in Genus und Numerus übereinstimmen, ganz genau geht es dabei um das Verhältnis zwischen es und dem lexikalischen Kopf der NP, das Kongruenz genannt wird. Man sagt auch, dass es und die NP hinsichtlich Genus und Numerus kongruieren. Andererseits gibt es auch Bezugnahmen auf NPs, bei denen eine solche Übereinstimmung fehlt. Die Frage nach der Kongruenz ist nur bei NP-Bezügen von Bedeutung, da bei Elementarsätzen oder anderen Wortgruppen (ADJPs oder VPs) die nominalen Kategorisierungen nicht in Frage kommen. Man kann ja nicht sagen, dass etwa einem Elementarsatz Kategorien wie Neut oder $S g$ zugewiesen werden können. Dies gilt auch für Adjektive, die in prädikativer Funktion in ihrer Grundform erscheinen.

Zunächst soll die Übereinstimmung bei NP-Bezügen präzisiert werden, dann werden kongruente und nicht-kongruente Bezugnahmen kommentiert. In Anlehnung an Eisenberg (2006: 33ff., 36f.) sollen hinsichtlich der Übereinstimmungsrelation Kongruenz und Rektion sauber getrennt werden. Rektion liegt nach Eisenberg vor, wenn eine syntaktische Wortkategorie des einen Elementes in der Rektionsbeziehung Formmerkmale des anderen bestimmt. So regiert Wein in der NP guten Wein in Sie mag guten Wein das Adjektiv hinsichtlich Genus, weil Genus beim Substantiv eine Wortkategorisierung ${ }^{45}$ darstellt (ebd., 36f.), was hier wiederum heißt, dass das Substantiv Wein hinsichtlich Genus invariabel ist. Bezogen auf es kann man in diesem Sinne sagen, dass der Antezedent, genauer sein lexikalischer Kopf, die Form der Anapher hinsichtlich Genus regiert. In einem Beispiel wie Ich habe ein neues Bett. Es ist sehr bequem. ist Neutrum eine nicht veränderbare Wortkategorie des Kopfnomens, die entscheidend ist für die Wahl der neutralen Anapher, also für die des es statt er oder sie. Was den Numerus angeht, so herrscht zwischen es und dem lexikalischen Kopf der Bezugs-NP Kongruenz. Dies bedeutet, dass der Numerus der Anapher von dem des Kopfnomens abhängt, wobei Numerus bei Nomina eine Einheitenkategorisierung darstellt. Möchte man Rektion und Kongruenz vereinheitlichen, so besteht - ebenfalls in Anlehnung an Eisenberg (2006: 38) - die Möglichkeit ihrer Zusammenfassung als Korrespondenz. Dieser Begriff steht vereinfachend dafür, dass Einheiten formal aufeinander abgestimmt werden. Grundsätzlich stellt sich dabei allerdings die Frage, ob die Eisenberg'sche Darlegung der syntagmatischen Relationen Rektion und Kongruenz für das Verhältnis zwischen es und Antezedent gilt, denn Eisenberg entwickelt

\footnotetext{
${ }^{45}$ Zur genauen Unterscheidung Wortkategorien vs. Einheitenkategorien vgl. Eisenberg (2006: 16f., 20).
} 
sein Konzept in erster Linie für Beziehungen zwischen Konstituenten. Es als Anapher und sein Bezugsobjekt bilden aber gemeinsam gewiss keine Konstituente. Trotzdem kann man auch in diesem Fall von Korrespondenz (also von Rektion und Kongruenz) ausgehen, weil syntagmatische Relationen nicht nur rein syntaktisch, also zwischen Konstituenten, zu fassen sind, sondern auf allen Ebenen des Systems (ebd., 33). So bezeichnet auch Harweg (1979: 25) das es (und sie und er) als zweidimensionales Substituens, das (auch) syntagmatisch substituiert, vgl. 2.1. Bei Eisenberg (2006: 168)

ist die Form des Pronomens bei phorischem Gebrauch von der Form eines anderen Nominals bestimmt. Fragt der Verkäufer »Welche Kiste wollen Sie denn?« und der Kunde antwortet »Ich nehme diese hier«, dann liegt phorischer Gebrauch vor: diese ist Fem Sg, weil welche Kiste FEM Sg ist. ${ }^{46}$

Die Kleinbuchstaben bei der Bezeichnung der Genuskategorie (Fem) deuten dabei in der Eisenberg'schen Konvention (ebd., 21) auf Einheitenkategorien hin, während Großbuchstaben (FEM) Wortkategorien markieren. Das heißt, die Wortkategorie FEM beim Nomen ${ }^{47}$ regiert die Einheitenkategorie Fem beim Pronomen. Dagegen kongruieren sie hinsichtlich Numerus.

Als Regelfall wird häufig angegeben, dass es NPs aufnimmt, deren lexikalischer Kopf ein neutrales Nomen im Singular ist, vgl.:

(49) Die 1. Gabi bekam von der Großmutter aus Chr. ein goldenes Armband, vielmehr Geld um es sich zu kaufen. (10) (K)

Die Bedingung der Korrespondenz ist jedoch weder bezüglich Genus, noch bezüglich Numerus zwingend, vgl.:

(50) Gott der Herr walte über euren kleinen, u. seid überzeugt, wenns erhalten werde, so wird er erhalten. (14) (L)

\footnotetext{
${ }^{46}$ Es wird an dieser Stelle davon abgesehen, ob dieser Gebrauch von dies tatsächlich phorisch und nicht eher deiktisch ist. Eisenbergs Beispiele und Ausführungen (ebd., 168, 170) sind meiner Meinung nach selbst nicht eindeutig. Wichtig ist aber in diesem Bezug die Korrespondenz zwischen Kiste und diese.

${ }^{47}$ Statt Nomen verwendet Eisenberg hier Nominal, das ein Sammelbegriff für deklinierbare Ausdrücke ist (ebd., 22), also auch Nomen umfasst.
} 
(51) Da ging es mit uns an ein Sieden und Braten, denn wir wurden gar knapp erhalten; wir buken so viel Pfannkuchen, Eierfladen \{et cetera\} und schleppten's mit heim, daß wir wohl vierzehen Tage davon essen konnten. (7) (MD)

(50) zeigt einen Verweis auf eine NP mit maskulinem lexikalischem Kopf, während in (51) weder Numerus noch Genus „stimmen“. Im letzteren Fall kann das es mit Harweg (1979: 220) als „fusioniertes mehrfaches Substituens“ bezeichnet werden, weil es aneinander gereihte Bezugsgrößen vereint und diese wie ein Ganzes wiederaufnimmt. „Echte“, d.h. nicht fusionierende, pluralische Bezüge sind aber auch vorstellbar:

(52) Kirschen dürfen wir essen, so viel mir wollen. Nur müssens wir selbst pflücken. (11) (SCHG)

Dieser Aspekt wird bei der Thematisierung von regulären NP-Wiederaufnahmen in der Fachliteratur praktisch ausgeklammert. Nicht-korrespondierende Verweise werden zwar mit berücksichtigt (vgl. etwa Askedal 1990: 214f., dort Typ 3 und 4), aber in aller Regel handelt es sich dabei um Gebrauchsweisen von es, bei denen entweder auf andere Phrasen als NPs Bezug genommen wird (Typ Peter ist fleißig, $d u$ bist es nicht), oder aber um spezielle Konstruktionen, etwa Kopulasätze mit es und einer anderen NP (vgl. (21) in 2.2.1). In (50)(52) sieht man aber, dass fehlende Korrespondenz auch bei Bezügen auf NPs anzutreffen ist. Im empirischen Teilkapitel (2.4) wird auf diese Fälle weiter eingegangen. An dieser Stelle sollen Kopulasätze des Typs es + Kopula $+N P$ noch kurz angesprochen werden, da sie m.E. eine besondere Form darstellen:

(53) Der Grabstein sieht jetzt geputzt schön aus, der Steinmetz sagt es wäre Marmor, (1954) (LB)

(54) fuhren bald vor bald hinter uns in die Erde, daß Stein und Rasen hoch in die Luft sprang bald mitten ein, und spickten uns die Leuthe aus den Gliedern weg, als wenn's Strohhälme wären. (2) (B)

In (53) liegt eine Bezugnahme auf eine NP vor, deren lexikalischer Kopf die Kategorie MASK trägt. In (54) ist die Form die Leuthe eine pluralische. Interessant ist die Konstellation es + Kopula $+N P$ in diesen und anderen ähnlichen Beispielen meiner 
Meinung nach vor allem deshalb, weil die Nicht-Korrespondenz vielfach zwingend ist oder zumindest bevorzugt wird, was aus der Funktion und dem Charakter der Konstruktion abzuleiten ist. Möglich sind zwar auch die korrespondierenden Varianten

(53’) Der Grabstein sieht jetzt geputzt schön aus, der Steinmetz sagt er wäre Marmor

und

(54') fuhren bald vor bald hinter uns in die Erde, daß Stein und Rasen hoch in die Luft sprang - bald mitten ein, und spickten uns die Leuthe aus den Gliedern weg, als wenn sie Strohhälme wären.

In diesem Fall entsteht jedoch nicht das spezifische Plus in der Bedeutung, das darin liegt, dass in Konstruktionen aus es + Kopula $+N P$ eine Charakterisierung oder Identifizierung im Mittelpunkt steht. Obige Belege repräsentieren Erstere, für Letztere sei folgendes Beispiel angeführt:

(55) Der Oberknecht, es war der älteste Sohn aus erster Ehe, sagte mir die Köchin, steht auf und betete laut mit allen zusammen ein ziemlich langes Tischgebet, katholisch. (10) (SCHG)

Askedal (1990: 214) spricht zusammenfassend von einer „nichtkongruierende[n] anaphorische[n] Identifizierungs/Charakterisierungskonstruktion":

Ihr kommt die Aufgabe zu, bereits im Text oder Diskurs eingeführte Referenten näher zu identifizieren. Ein Standardkontext ist das Frage-Antwort-Paar, mit dem die Identität einer Person oder einer Sache erfragt werden soll: „Wer/Was ist es/X? - Es ist Y.“ In der Antwort erscheint die Angabe zur Identifizierung als 'rhematisches Komplement'. (Zifonun 2001: 77)

Diesem rhematischen Element, das als NP erscheint (Marmor, Strohhälme), geht das es als neutrales, thematisches Element voraus. Es nimmt die eingeführte Einheit zunächst unspezifisch auf, was die Voraussetzung dafür ist, dass der Akzent auf die Charakterisierung oder Identifizierung gelegt werden kann. Diese unspezifische 
Wiederaufnahme scheint zudem mit den Überlegungen Vogels (2006: 78) zu Existenzverben, u.a. Kopulaverben, zu korrelieren. Demnach drücken diese Verben eine Geschehenssemantik aus ${ }^{48}$, die für so genannte thetische Sätze typisch ist (ebd., 69). Diese wiederum weisen oft ein es auf (es kommen Gäste zu uns, es wird getanzt), das in solchen Sätzen als Thetizitätsmarker betrachtet werden kann (ebd., 69, 78, 92f., 117, 245f., so auch Scheibl 2001). Wenn dem so ist, so würde dies bedeuten, dass ein grundsätzlich auf das nicht-phorische es zugeschnittenes Konzept auch in den phorischen Bereich Eingang findet. Dies wiederum steht im Einklang mit der Zielsetzung dieser Arbeit, Zusammenhänge zwischen den einzelnen es-Gebrauchsweisen sichtbar zu machen. Die Bevorzugung von neutralem es in charakterisierenden Konstruktionen könnte nach dieser Logik darauf zurückgeführt werden, dass es kraft seiner Funktion als Thetizitätsmarker sehr gut zu Kopulaverben mit Geschehenssemantik passt und in (53)-(55) dies zum Ausdruck kommt. Der Begriff der Thetizität soll bei der Beschreibung des nicht-phorischen es wieder aufgegriffen werden, s. dazu weiterhin Kapitel 2.4.

Auch andere Phrasentypen als NPs kommen in Frage, wenn phrasale Verweise durch es thematisiert werden. So finden wir Adjektivphrasen (ADJP) mit einem Adjektiv als lexikalischem Kopf in Fällen, in denen auf Prädikative in Kopulasätzen Bezug genommen wird:

(56) Doch ist sie nur so schön u. ich bin es nicht. (108) (SCH)

Hier ist Korrespondenz zwischen es und seinem Bezugsausdruck in Genus und Numerus offensichtlich nicht gegeben, da Adjektive in prädikativer Funktion hinsichtlich dieser Kategorisierungen nicht spezifiziert werden.

Zusammenfassend werden für explizite Phorik durch es folgende morphosyntaktischen Kategorien angenommen:

Bezugsgröße kategorial nicht erfassbar

Bezug auf Elementarsätze

Bezug auf Phrasen

${ }^{48}$ Das Pendant hierzu ist die Handlungssemantik von Verben wie tanzen oder schlagen, bei denen ein Agens als Beteiligter erscheint. 
- Bezug auf VPs

- Bezug auf NPs

- Bezug auf ADJPs

Vor allem das Bild über den Bezug auf Elementarsätze wird noch zu differenzieren sein, weil der syntaktische Rahmen solcher Bezugnahmen unterschiedlich aussehen kann.vgl. (41)-(43). In erster Linie soll dabei das Korrelat-es (s. etwa (46)) eingehend untersucht werden.

\subsection{Gebundene Phorik: Das Korrelat-es}

In diesem Kapitel wird die dritte Phorikart, nämlich die syntaktisch gebundene thematisiert. Die Überlegungen orientieren sich dabei an folgenden Fragen:

- Wie kann der Gebrauch des es als Korrelat innerhalb des bisher behandelten Systems der es-Phorik verortet werden? Was bedeutet dabei „syntaktisch gebunden“? (2.3.1)

- Wie können das Korrelat-es und das Verhältnis zwischen es und korrelierter Einheit beschrieben werden? (2.3.2)

- Wie lässt sich das Korrelat-es mit dem nicht-korrelativen Verweis auf Elementarsätze verbinden? (2.3.3)

\subsubsection{Kataphorik und Gebundenheit}

In 2.1 habe ich zu der Annahme, Kataphern seien einfache Umkehrungen von Anaphern (IDS-Grammatik 1997: 548, Schmidt 1987: 97, Harweg 1979: 54), kritisch Stellung genommen. Ich habe kurz darauf hingewiesen, dass kataphorische Verweise nicht die gleiche Art von Phorik darstellen können wie anaphorische, da zwischen ihnen ein wesentlicher funktionaler Unterschied besteht, indem Kataphern die Orientierungskontinuität nicht aufrechterhalten, sondern antizipieren und die 
Aufmerksamkeit auf das zu Thematisierende lenken. Dies bedeutet gleichzeitig, dass durch Kataphern nicht auf bereits Eingeführtes verwiesen wird. Folglich kann bei dieser Art von Phorik nicht das herkömmliche anaphorische Verhältnis zwischen Substituendum und Substituens vorliegen. Überhaupt stellt sich die Frage beim Korrelat-es, ob es tatsächlich phorisch ist. In der Tat sind in diesem Zusammenhang differierende Positionen anzutreffen: Für die Duden-Grammatik (2005: 831) und Zifonun (1995: 42) ist das Korrelat-es expletiv bzw. nicht-phorisch. In der IDS-Grammatik finden sich unterschiedliche Feststellungen, die auf Schwierigkeiten bei der Bestimmung dieses es-Gebrauchs schließen lassen. So liest man einerseits (1997: 38), dass dieses es als expletiv einzustufen ist. An einer anderen Stelle wird diese Zuordnung insofern abgeschwächt, als die Rückführbarkeit des Korrelat-es auf den kataphorischen Gebrauch angedeutet wird (ebd., 549). Dann ist in dem Kapitel zu Korrelaten zu lesen: „Korrelate sind somit Formen KM-internen ${ }^{49}$ phorischen oder deiktischen Bezugs“ (ebd., 1475) sowie „Bei der hier präsentierten Lösung wird das Korrelat [...] als geeignet zum referentiellen Gebrauch eingestuft.“ (ebd., 1489) und „Daß das Korrelat-es in der Tat referentiell verwendet werden kann und nicht nur strukturbedingter Platzhalter ist $[\ldots]$ (.. (ebd., 1490). ${ }^{50}$ Die beiden letzten Aussagen lassen sich meiner Meinung nach so interpretieren, dass das Korrelat-es zwar grundsätzlich nicht phorisch ist, dabei jedoch die Möglichkeit nicht ausgeschlossen wird, es phorisch zu deuten, vgl. auch das Beispiel auf Seite 1490. U.a. diese schwere Bestimmbarkeit führt in der vorliegenden Arbeit dazu, das Korrelat-es im Kontext „echter“ Verweise auf Sätze zu betrachten, was auch in der IDS-Grammatik (ebd., 1489, s. die Beispiele dort) kurz kommentiert wird. Die angesprochenen Probleme bei der Bestimmung des Korrelat-es legen es nahe, nach einer Art Goldener-Mittelweg-Lösung zu suchen. So schlägt Schmidt (1987: 107) vor, beim Korrelat-es von Phorik, nicht aber von Substitution zu sprechen. Grammatisch kann diesem Umstand Rechnung getragen werden, wenn in Anlehnung an Zifonun (2001: 76, 78) „ein rein struktureller Verweis innerhalb des Satzes“ („KM-intern“) angenommen wird. Strukturell bedeutet demnach, dass der Verweis nicht zwischen zwei, voneinander unabhängigen Sätzen hergestellt wird, sondern im Rahmen eines zusammengesetzten Satzes, also zwischen Haupt- und Nebensatz erfolgen muss, weshalb der Terminus syntaktisch gebunden gewählt wurde. Dies entspricht der herkömmlichen Definition von Korrelaten, die besagt, dass Korrelate den Nebensatz im Hauptsatz vertreten

\footnotetext{
${ }^{49} \mathrm{KM}=$ Kommunikative Minimaleinheit

${ }^{50}$ Es wird an dieser Stelle davon abgesehen, dass mit referentiell in diesen Aussagen wohl das gemeint ist, wofür in dieser Arbeit phorisch steht.
} 
(s. Sonnenberg 1992: 13). Beim Korrelat-es führt die Gesamtheit folgender Charakteristika zu der Annahme einer eigenen Art von Phorik:

- Kataphorik

- Gebundenheit an ein Satzgefüge (morphosyntaktische Umgebung)

- Phorik, aber keine Substituenshaftigkeit

- Grammatische Besonderheiten

Der letzte Punkt bildet das Thema des nachfolgenden Kapitels.

\subsubsection{Grammatik der es-Korrelate}

Korrelate werden in der älteren Fachliteratur im Wesentlichen gleichzeitig syntaktisch und semantisch bestimmt. Blatz (1896: 779) definiert sie wie folgt:

Häufig stehen im Hauptsatze besondere Wörter demonstrativer Natur, welche die Beziehung des Nebensatzes auf den Hauptsatz, sowie das Gedankenverhältnis, in welchem der Nebensatz zum Hauptsatze steht, andeuten. Man nennt diese Wörter die determinativen Korrelate, oder schlechthin die Korrelate des Nebensatzes. (Hervorhebungen von mir, D.C.)

In neueren Arbeiten werden jedoch Syntax und Textsemantik - dem jeweiligen theoretischen Rahmen und den jeweiligen Zielsetzungen entsprechend - häufig getrennt, bzw. es wird ein Aspekt hervorgehoben, unter dem dann der Terminus Korrelat definiert wird. So begegnet man - wie der ausführliche Überblick der Terminologie in Sonnenberg (1992: 4f.) zeigt - eher semantisch orientierten Begriffen wie "Hindeutung" auf der einen bzw. eher syntaktisch motivierten Begriffen wie "Formwort" auf der anderen Seite, wobei jedoch auch Termini wie "Bezugswort" oder "Stützwort" (ebd.) anzutreffen sind, die hinsichtlich der Begriffsbestimmung eher neutral ausfallen. In der neueren Grammatikschreibung ist die Tendenz zu sehen, dass Korrelate in erster Linie syntaktisch bestimmt werden. So spricht Sonnenberg (1992: 13) von „Fügungselementen für den untergeordneten Satz im übergeordneten Satz“ bzw. (1992: 143) von „Fügungselemente[n], 
die den Anschluß eines untergeordneten Teilsatzes an einen Valenzträger im Obersatz herstellen“. Die Berücksichtigung syntaktischer und (textuell)-semantischer Argumente spielt bei der Beschreibung der Funktion von Korrelaten - darunter des es - auch dann eine wichtige Rolle, wenn die Frage beantwortet werden soll, wann ein Korrelat obligatorisch erscheinen oder fehlen muss bzw. fakultativ stehen kann. Zusammenfassend kann hier in Anlehnung an die Darstellung in der Duden-Grammatik (2005: 1064ff.) festgehalten werden, dass bei der Setzung bzw. Nicht-Setzung eines Korrelates folgende Faktoren wirksam sind: ${ }^{51}$

\section{Lexikalischer Faktor}

Die Wahl des Verbs bestimmt die Setzung bzw. Nicht-Setzung des Korrelates (Pütz 1975: 69, Dalmas 1996: 31f.). Bezogen auf es kann man hier zwischen einer älteren, dichotomisch ausgerichteten (Köhler 1976: 227f.) und einer neueren, skalar motivierten Auffassung unterscheiden. Geht man, wie Pütz (1975, Kapitel 3.2.5), dichotomisch vor, so nimmt man eine Zweiklassengesellschaft an, in der es zwei Verbklassen gibt. Die eine Klasse umfasst Verben, bei deinen kein zugrundeliegendes Korrelat ${ }^{52}$ angenommen wird (1975: 70), bei denen also obligatorisch kein Korrelat-es erscheint. Der anderen Klasse werden Verben zugeordnet, die in ihrer tiefenstrukturell angenommenen Subjekt- oder Objektsatz-NP eine es-Größe enthalten, bei denen also ein Korrelat-es obligatorisch erscheinen muss (1975: 68). Konzipiert man jedoch die Regelhaftigkeiten bei der Setzung- und Nicht-Setzung des Korrelat-es skalar (vgl. Duden-Grammatik 2005: 1065 und IDS-Grammatik 1997: 1483), so kann man von Tendenzen sprechen: „Bei bestimmten Verben (bzw. Adjektiven und Substantiven) besteht eine starke Tendenz, ein Korrelat zu setzen, bei anderen nur eine schwache oder gar keine.“ (Duden-Grammatik 2005: 1064) Auf diese Weise kommt man zu der Schlussfolgerung, dass es Verben gibt, die „normalerweise mit Korrelat“ bzw. „normalerweise ohne Korrelat“ stehen (ebd.). Zwischen diesen beiden Polen stehen dann Verben, bei denen Schwankungen festzustellen sind, was den Korrelatgebrauch angeht.

\footnotetext{
${ }^{51}$ Für weitere Literatur s. Pütz (1975, 1990), Köhler (1976), Ballweg-Schramm (1976), Ulvestad/Bergenholtz (1979, 1983), Latour (1981), Marx-Moyse (1983), Sadzinski (1983), Cato Hoff Lambine (1997), Sandberg (1998), Zitterbart (2002), Boszák (2009).

${ }^{52}$ Entsprechend der Begrifflichkeit der 1975 gültigen Version der generativen Grammatik spricht Pütz hier davon, dass kein Korrelat-es in der Tiefenstruktur des jeweiligen Verbs, genauer in dessen Subjekt- oder Objektsatz-NP erscheint. Damit geht er einen anderen Weg als seine Vorgänger (s. die Diskussion der früheren Fachliteratur, 1975: 65ff.), die davon ausgegangen waren, dass ein Korrelat-es bei Verben mit Subjekt- bzw. Objektsatzforderung tiefenstrukturell immer generiert, dann aber manchmal (d.h. bei bestimmten Verben) während des Zustandekommens der Oberflächenstruktur getilgt wird.
} 
Sowohl die Tatsache, dass eine Zwischenstufe (,schwankend“) angenommen wird, als auch die Wortwahl („,normalerweise“) deuten darauf hin, dass es um Obligatorik und Fakultativität des Korrelates komplizierter bestellt ist, als dass man zwei, eindeutig ${ }^{53}$ abgrenzbare Klassen etablieren könnte. Erweitert wird diese Perspektive auch dadurch, dass als Hauptsatz fungierende Kopulasätze mit einem Adjektiv des Typs Es ist möglich, dass... von Vollverbkonstruktionen getrennt analysiert werden können (Zitterbart 2002a: 84), weil die jeweiligen Subjektsätze in diesen Fällen nicht von Verben, sondern von Adjektiven oder Substantiven in Verbindung mit Kopulas selegiert werden. Auch Pütz (1975: 23) formuliert eigene Regeln für adjektivische Fälle. Andererseits weist Zitterbart (2002a: 77) selber darauf hin, dass das Verhalten des Korrelat-es in von Vollverben geforderten Subjektsätzen „sich von jenem in Kopulakonstruktionen sehr geringfügig [unterscheidet].“ Eine andere Erweiterung des semantischen Faktors zeigt die Feststellung Günthners zu Belegen in gesprochener Sprache (2009: 24, 43), nach der in erster Linie evaluative, epistemische und evidenzielle Prädikatsausdrücke ein Korrelat bei sich haben.

\section{Grammatisch-semantischer Faktor}

Wie Ulvestad/Bergenholtz (1979: 105) gezeigt haben, scheint das Auftreten eines esKorrelates nicht nur vom jeweiligen Verb abzuhängen, sondern auch davon, welche grammatische Konstellation im jeweiligen zusammengesetzten Satz vorliegt. So sind es u.a. der Typ des Junktors, Tempus und Modus des Hauptsatzes und die Stellung des Nebensatzes, die einen Einfluss darauf haben, ob ein es als Vetreter des Nebensatzes im Hauptsatz erscheint oder nicht. Dabei ist die Position des Nebensatzes als absolut sicherer Anhaltspunkt anzusehen, indem in der Fachliteratur allgemein bekannt ist, dass die Bewegung des Nebensatzes ins Vorfeld des übergeordneten Satzes dazu führt, dass kein Korrelat-es erscheinen darf, vgl. z.B. Duden-Grammatik (2005: 1065). Ulvestad/Bergenholtz (1983: 21) erwähnen auch Gradpartikeln wie nur, nur noch, sogar, auch, die auf den Korrelatgebrauch restringierend wirken. ${ }^{54}$ Eisenberg (2006: 330) betont

\footnotetext{
${ }^{53}$ Pütz (1975: 68-80) untermauert seine Annahmen mit Hilfe syntaktischer Tests.

${ }^{54}$ Cortès (2003: 142) geht weiter und behauptet, dass vor dem korrelierten Nebensatz keine Modal- oder Gradpartikeln stehen dürfen, was die enge Beziehung zwischen Haupt- und Nebensatz zeige. Auch das es vetrage sich mit solchen Partikeln nicht (ebd., 156). Zu einem anderen Ergebnis kommt Günthner, die (2009: 24f., 34) argumentiert, dass Modalpartikeln und andere Modalitätsmarker im korrelathaltigen Teil häufig vorkommen, da sie den evidenziellen, epistemischen und evaluativen Charakter der beteiligten Verben unterstreichen. Das Problem besteht dabei m.E. darin, dass alle angeführten Belege von Günthner ein esKorrelat enthalten, das am Satzanfang steht. Diese Position ist für das es jedoch immer verfügbar, deshalb ist es nicht möglich, die Feststellung Günthners mit den gegenteiligen Thesen etwa bei Ulvestad/Bergenholtz $(1979,1983)$ zu kontrastieren.
} 
neben der lexikalischen Bestimmung der Korrelatsetzung auch den Grammatikalisierungsgrad bei der Besetzung von Komplementstellen: „Je weniger eine Komplementposition für Sätze syntaktisch festgeschrieben ist, desto eher braucht sie es zur Anbindung des Satzes. Das verträgt sich gut mit der Beobachtung, dass es bei nichtkanonischen Komplementsätzen, z.B. den wenn-Sätzen, eine wichtige Rolle spielt.“

\section{Informationsstruktureller Faktor}

Neben den oben erwähnten lexikalischen und grammatischen Steuerungsmechanismen des Korrelatgebrauchs ist es eine allgemein akzeptierte Erkenntnis in der Fachliteratur, dass die Setzung eines Korrelat-es im Rahmen der funktionalen Satzperspektive geregelt wird. Zur Vermeidung des Korrelatgebrauchs kommt es demnach, wenn durch den Nebensatz neue Information eingeführt wird oder wenn es den Textverlauf stören würde (Beispiele hierfür in Zitterbart 2002b: 189f.), wohingegen das Auftreten eines Korrelates im Wesentlichen auf einen thematischen Nebensatz zurückzuführen sei. Was den ersteren Aspekt (neue Information $\rightarrow$ kein Korrelat, bekannte Information $\rightarrow$ Korrelat) angeht, so ist er nicht unbekannt. Bereits Kemme (1979: 20) hat darauf in seiner didaktisch orientierten Arbeit hingewiesen. Wird ein Korrelat gesetzt, dann gilt: „Er [der im Nebensatz beschriebene Inhalt, D.C.] mag vorher behauptet worden sein, oder der Sprecher unterstellt anderen, sie hätten ihn gedacht, vorgestellt o.ä.“. Das es kündigt also einen thematischen Nebensatz an, dessen Sachverhalt bekannt ist, über den im Hauptsatz etwas Neues, Wesentliches, d.h. Rhematisches ausgesagt wird.

Wie aus den obigen Überlegungen ersichtlich, werden bei der Beschreibung der Funktion von Korrelaten sowohl textsemantische als auch syntaktische Gesichtspunkte herangezogen. Für alle diese Bereiche gilt, dass - abgesehen von wenigen Ausnahmen, bei denen absolute Notwendigkeit oder eindeutige Nicht-Setzung des Korrelat-es angenommen werden kann (s. etwa Zitterbart 2002a: 84, 96) - lediglich Tendenzen beobachtbar sind, was Setzung und Fehlen des Korrelates angeht. Absolute Gültigkeit wird praktisch nie erreicht, da auch für zu 100\% geltend gehaltene Regeln und auch Korpusdaten immer wieder widerlegt bzw. Gegenbeispiele gefunden werden können, vgl. Zitterbart (2002a: 73) und IDS-Grammatik (1997: 1483). In der vorliegenden Arbeit möchte ich mich daher nicht auf Obligatorik und Fakultativität des Korrelats konzentrieren. Stattdessen soll dieser Bereich der $e s$-Forschung in eine teilweise andere Richtung erweitert werden, indem in einem ersten Schritt das Korrelat und sein Verhältnis zum korrelierten Nebensatz beschrieben und im 
Anschluss daran (2.3.3) nicht-korrelative Bezüge auf Elementarsätze mit berücksichtigt werden.

Die wichtigste grammatische Frage scheint die nach dem kategorialen Status des Korrelat-es zu sein. Ganz genau geht es dabei darum, wie das Verhältnis zwischen Korrelat und Bezugseinheit (Satz) zu beschreiben ist. Auf den ersten Blick hat man es nämlich mit der Doppelbelegung einer einzigen Komplementstelle von Verben oder anderen Prädikatsausdrücken zu tun, ${ }^{55}$ wobei Subjekte und direkte Objekte als Komplemente in Frage kommen: ${ }^{56}$

(57) ich sagte kein wort und verlangte keine Erdbiren | aber bitter war es mir, mich auf diese art von meiner mutter behandelt zu sehen (11) (DM)

(58) da beij hielt ich sehr streng auf tugend und Rechtschafenheit | und so wagt Es niemand mir durch das gegentheil zu nahe zu treten. (1) (DM)

Wenn man der „These“ der Doppelbelegung folgt, so kann man ein Dependenzverhältnis zwischen Korrelat und Korreliertem erwägen. Nach Eisenberg (2006: 178)

ist es nicht eine selbständige Ergänzung, sondern bildet gemeinsam mit dem extraponierten Ausdruck [dem Nebensatz oder der IK, D.C.] das Subjekt [bzw. ggf. das Objekt, D.C.]. [...] Dies kommt in der Konstituentenstruktur zum Ausdruck, wenn man dem Subjekt intern die Struktur ähnlich einer Attributkonstruktion gibt.

Dies würde bedeuten, dass das Untergeordnete Attribut zu es ist. Gegen diese Analyse spricht, dass nicht das es, sondern der jeweilige Prädikatsausdruck entscheidet, ob im gegebenen Fall eine IK oder etwa ein dass- oder $o b$-Satz gesetzt wird. In der Tat favorisiert Eisenberg an einer anderen Stelle (ebd, 330) eine andere Lösung. Er schreibt es Kopffunktion zu, weil es ,gewisse grammatische Merkmale der jeweils besetzten Position trägt (es ist eine Pronominalform des Nom/Akk).“ Dabei argumentiert er jedoch im

\footnotetext{
$\overline{{ }^{55} \text { Vgl. auch Kay }(2007: 1}$, 4) für englisches it in gleicher Funktion: „We can think of such situations as involving double instantiation. A particular sentential argument is instantiated in an ordinary syntactic position with a semantically empty constituent (in all cases the word "it") and it is also instantiated later in the sentence, in the form of an expression which gives its semantics.“ und „IT-Extraposition belongs to a class of constructions in which single valence elements are realized as two different constituents of the actual sentence."

${ }^{56}$ Gelegentlich ist auch davon die Rede, dass es Gliedsätze korreliert (Cortès 2003).
} 
Gegensatz zu seiner Position auf Seite 178 dafür, „dass das Korrelat nicht referentiell und damit nicht als Kern einer Attributkonstruktion angesehen werden kann.“ (ebd.) Problematisch sind auch die abgebildeten Konstituentenstrukturen, weil diejenige, die zuerst (ebd., 178) dem Satz mit Korrelat-es zugewiesen wird, später (ebd., 330) auf die Verwendung von das bezogen wird, das in einem Satz wie Das weiß er, ob du fährst nicht nur als Kopf, sondern auch als Kern fungiert (ebd.). Der Unterschied ist wichtig, denn während es und Nebensatz in einem S-Knoten ${ }^{57}$ zusammengefasst werden, bilden das und Nebensatz eine Nominalgruppe, was aus der Attributkonstruktion ja folgt. Eine andere Meinung vertritt Eroms (2000: 200f.), der Interdependenz zwischen Korrelat und Nebensatz annimmt. Interdependenz signalisiert, dass in einer gegebenen Relation „keine einfache und einsträngige Bezüglichkeit““ (ebd., 253) herrscht. Das Paradebeispiel hierfür ist die Verbindung eines Nomens mit einem Artikel, bei der die Operatoraufgabe des Artikels, das Nomen im Diskurs einzuordnen, für seine Regensqualität spricht, während jedoch die „unbestreitbaren Kopfeigenschaften des Substantivs“ (ebd.) ein Signal dafür sind, dass das Nomen als Regens fungiert. In NPs sind die Verhältnisse kompliziert, wie Eroms (ebd.) dies ausführt:

Der Artikel bildet nun keine direkte dependentielle Zwischeninstanz zwischen Verb und Substantiv [das Substantiv hängt dabei vom Verb ab, D.C.], sondern spezifiziert mit seinem lexikalischen Teil das Substantiv, der morphematische Teil ist darstellungslogisch mit dem Substantivmorphem redundant, de facto aber stiftet er zumeist die Eindeutigkeit der Bezüge. [...] Der Artikel ist eine auf das Substantiv hingeordnete spezifische Morphemkombination mit Wortstatus, die man em ehesten durch Interdependenzen zwischen den beiden Wörtern erfassen kann.

Ähnliche Bezüge werden (ebd.) auch zwischen sich und Verb bei medialen Verben wie sich wundern angenommen (ebd., 200). Die Frage ist nun, ob der Interdependenzbegriff, der bei Eroms nur bezogen auf NPs eingehend analysiert wird und der ,multifunktionale morphematische Relationen“ (ebd., 253) widerspiegelt, auch für Relationen zwischen anderen Einheiten geeignet ist. Was Korrelate angeht, so findet man (ebd., 200) Strukturdiagramme zu Satzgefügen, in denen Präpositionaladverbien als Korrelate auftreten (z.B. Ich wundere mich darüber, dass er kommt), wobei zwischen dem Korrelat und dem korrelierten Nebensatz Interdependenz angesetzt wird. Hier könnte man argumentieren, dass die Form des Korrelates die zum Verb gehörige Komplementklasse eindeutig anzeigt, eine ${ }^{57} \mathrm{~S}=$ Satz 
Kopfeigenschaft also vorliegt. Andererseits erfolgt die inhaltliche Füllung erst durch den Nebensatz. Allerdings ist dieser Gedankengang auf es-Korrelate nicht ohne Weiteres übertragbar. Der Grund dafür ist, dass es formal nicht kasusmarkiert ist. Sowohl im Akkusativ als auch im Nominativ heißt es eben es. Die rektionalen Eigenschaften sind hier also nicht erkennbar. Man kann nicht sagen, dass es gewisse grammatische Merkmale der zu besetzenden Komplementstelle trägt. Streng genommen kann man erst durch den Prädikatsausdruck entscheiden, ob es als Subjekt oder als Objekt zu deuten ist. Formal wird dies jedoch nicht markiert. Die einzige Ausnahme scheint der Fall zu sein, wenn das Korrelat-es am Satzanfang steht. Dort kann nämlich kein Objekt-es vorkommen. Auch das ist aber keine formale Kennzeichnung, sondern eine topologische. Die Annahme von Interdependenz ist also in Analogie zu Präpositionaladverbien zwar begründet, formal jedoch nicht zu rechtfertigen. In Czicza (2003: 42) habe ich dafür argumentiert, dass es sich bei Sätzen mit es-Korrelat um die diskontinuierliche Realisierung einer Komplementstelle handelt. Mit dieser Ansicht kompatibel ist die Position der IDS-Grammatik, in der nicht einfach Korrelate, sondern Korrelatverbindungen (KV) als eigene Kategorien angenommen werden (1997: 1488). So lauten (ebd.) die allgemeine Regel und die Definition wie folgt:

Termkomplemente, die durch IK oder SJS [Sätze, eingeleitet durch einen Subjunktor, D.C.] sowie den Regeln entsprechend immer durch phorische und deiktische Ausdrücke - belegt sein können, können auch durch KV belegt sein. Elemente der Kategorie KV sind Paare, bestehend aus einem phorischen/deiktischen Ausdruck und einer IK oder einem SJS, die in der Kette diskontinuierlich und gegebenenfalls akzentuiert auftreten können.

Diese Lösung ist darauf zurückzuführen, dass dadurch der phorische Charakter von Korrelaten erkennbar bleibt und gleichzeitig zu sehen ist, dass der Nebensatz oder die IK vom Verb (oder von einem anderen Prädikatsausdruck) gefordert wird. Die Stärke der Definition liegt in der Formulierung belegt sein können, denn es besagt, dass KVen als Belegung von Komplementstellen prinzipiell immer in Frage kommen. Wenn also ein Prädikatsausdruck festlegt, dass eine seiner Komplementstellen durch einen dass-Satz oder eine IK zu füllen ist, so folgt daraus automatisch, dass diese sententialen Füllungen auch in Form von KVen erscheinen können. Allerdings ist diese Optionalität auch die Schwäche der Definition, weil die Fragen Wann? und Wie? nicht beantwortet werden. M.a.W.: Wie kann man die Vorkommensregelungen bei KVen grammatisch bestimmen? Wie oben erläutert 
wurde, ist die Antwort in diesem Fall keine rein grammatische, sondern eine, die auch auf andere (etwa pragmatische) Faktoren zurückgreifen muss. Hinzu kommt, dass die Bestimmung der KV als ein Paar aus zwei Einheiten m.E. nicht ausschließt, dass die beiden Einheiten ein Verhältnis zueinander aufbauen und dadurch eine gewisse Arbeitsteilung geschaffen wird (vgl. auch Günthner 2009: 29). So kann man davon ausgehen, dass das satzförmige Komplement den Inhalt „liefert“, während das Korrelat als Platzhalter dient (ebd., 1478, so auch Zifonun 2001: 126). ${ }^{58}$ Eine valenztheoretische Weiterführung dieser Position findet sich in dem Kapitel zum nicht-phorischen es. Weiterhin ist in diesem Zusammenhang interessant, warum überhaupt KVen erscheinen, was also ihre Vorzüge gegenüber korrelatlosen satzförmigen Komplementbesetzungen sind. Allgemein gilt (ebd., 1476):

Durch die Setzung des Korrelates wird die Satzstruktur morphologisch durchsichtiger und bietet der kognitiven Verarbeitung eine deutliche Unterstützung. Hinzu kommen weitere funktional nutzbare Eigenschaften, die sich aus der (nur teilweise vorhandenen) Akzentuierbarkeit des Korrelates, der Trennbarkeit der beiden Teile und der so gewährleisteten topologischen Variabilität ergeben. ${ }^{59}$

Beim es entfallen jedoch sowohl morphologische Durchsichtigkeit (s. oben) wie auch Akzentuierbarkeit (ebd., 1477) und topologische Variabilität (ebd., 1478). ${ }^{60}$ Es ist nämlich nicht betonbar und seine Stellung ist mit Bezug auf die korrelierte Einheit fest. Diese Festigkeit besteht darin, dass das Korrelat es und die korrelierte Einheit nur Distanzstellung aufweisen können (ebd.), vgl.:

*Es, dass du kommst, ist schön.

*Würdest du es, dass sie nicht kommt, bedauern?

\footnotetext{
${ }^{58}$ Eine moderne Version dieses Gedanken ist die Auffassung Günthners (2009: 20, 23), nach der KVen ein Fall von Projektorkonstruktionen sind. Der Begriff der Projektion geht auf Auer (2000) zurück und besagt im Falle der KVen, dass das Korrelat die korrelierte Einheit antizipiert und dabei im Hörer/Leser „Erwartungen bzgl. der weiteren Entwicklung des syntaktischen Musters aufbaut.“ (Günthner 2009: 20). Dabei meint diese Art von Projektion sowohl syntaktische als auch semantische Erwartungen, indem Subjekt- oder Objektsätze aufgebaut und semantische Gehalte erwartbar gemacht werden (ebd., 21).

${ }^{59}$ Vgl. hierzu auch Dalmas (1996: 29).

${ }^{60}$ Bleibt noch die These von der kognitiven Stütze, die in der vorliegenden Arbeit jedoch empirisch nicht überprüft werden kann.
} 
Distanzstellung kann auf der Folie ihres Paares, der Kontaktstellung, erläutert werden, sie ist ihr Gegenteil: „Bei Kontaktstellung folgen Korrelat und IK/Satz ohne Pausierung linear unmittelbar aufeinander und gehören demselben Stellungsfeld an.“ (ebd., 1477) Es und korrelierte Einheit dürfen also nicht in demselben Stellungsfeld stehen. Was Topologie angeht, so ist noch auf einen wichtigen Umstand hinzuweisen: Stellt die KV die Belegung der direkten Objektstelle eines Verbs dar, so kann das Korrelat nicht am Satzanfang stehen, weil akkusativischem es diese Stellung grundsätzlich verschlossen bleibt. Es muss im Mittelfeld erscheinen, wenn es überhaupt gesetzt wird. Diese Regelung gilt für die Belegung der Subjektstelle durch ein es-Korrelat nicht, es kann sowohl im Vor- als auch im Mittelfeld auftauchen. Interessant ist dabei, dass die eingeschränktere Variante, nämlich die einzig mögliche Mittelfeldposition des akkusativischen es, das zentrale, kommunikativ unmarkierte Muster bei KVen darstellen soll (ebd., 1479). Unterstützt wird diese These dadurch, dass diese topologische Regelung auch für durch Präpositionaladverbien eingerichtete KVen gilt und die Setzung des Korrelates im Mittelfeld die grammatisch bedingte Folge sei (ebd.). Entsprechend dieser Argumentation stellt die Anfangsstellung des subjektischen es-Korrelates „eine Variante des zentralen Musters“ dar (ebd.). Des Weiteren folgt aus diesen Überlegungen, dass akkusativische es-KVen ,typischere“ Fälle des Korrelatgebrauchs sind und demnach präpositionalen KVen in dieser Hinsicht näher stehen als nominativischen es-KVen. Auch Eisenberg (2006: 329) nennt es-Korrelate in Subjektfunktion am Satzanfang eine Besonderheit unter den Korrelaten. Zitterbart (2002a: 58ff.) weist darauf hin, dass mit dem Korrelat-es am Satzanfang ein Grenzfall syntaktischer Ambivalenz vorliegt, weil man nicht eindeutig entscheiden kann, ob es ,reines“ Vorfeld-es oder Korrelat ist. Die Frage ist nur, wie Vorfeldstellung als eine Variante der Mittelfeldstellung angesehen werden kann, wenn bezüglich der es-Setzung beide doch so unterschiedlich sind. Hinzu kommt, dass die angebliche Variante des zentralen Musters bei subjektischen es-KVen viel häufiger vorkommt als das zentrale Muster mit Mittelfeldstellung. Theoretisch ließe sich auch fragen, ob die Zuordnung der akkusativischen es-KV zum zentralen Muster mit Mittelfeldstellung nicht eher daraus folgt, dass dieses es ja im Vorfeld gar nicht stehen und deshalb automatisch auch nicht anderswo als im Mittelfeld erscheinen kann, sogar muss. Dieses Merkmal gilt aber nicht nur für das Korrelat-es in Objektfunktion, sondern für alle anderen es-Vorkommen in Objektfunktion. Meiner Meinung nach besteht also die Besonderheit des Subjektkorrelates nur darin, dass es mit anderen Vorfeldbesetzungen durch es verglichen werden kann, was für akkusativisches 
es nicht gilt. M.a.W.: Hinsichtlich der Funktionsweise von es-Korrelaten sollte zwischen Subjekt- und Objekt-es ${ }^{61}$ kein Unterschied gemacht werden. Die bestehenden Differenzen sind topologischer Natur, die in erster Linie das akkusativische es betreffen: Es kann nicht im Vorfeld stehen.

Der Grund ist wohl am ehesten darin zu sehen, daß hier eine so schwach ausgeprägte Form in einer - wenn auch relativ gering - markierten syntaktischen Funktion, als Akkusativkomplement, auftritt. Das heißt, eine strukturell markierte Einheit braucht offensichtlich auch ein gewisses Minimum an morphologischer und phonetischer Substanz, um an exponierter Stelle am Satzanfang stehen zu können. (IDS-Grammatik 1997: 1585)

Zusammenfassend kann man auf Grund der obigen Überlegungen sagen, dass das Korrelates a) nur in Distanzstellung zur korrelierten Einheit vorkommt, b) ein reiner Platzhalter in KVen ist, der dazu dient, die syntaktische Position, in der das jeweilige Komplement stehen würde, zu besetzen und der c) die Rhematisierung des vertretenen Komplements ermöglicht. In diesem Sinne spricht Cortès (2003: 151) von „Rhematisierungsmarkierern“. Rhematisierung kann erreicht werden, indem der satzförmige Teil, der den Inhalt liefert, in eine Position kommt, die für satzförmige Inhalte ideal ist: das Nachfeld. „Der Besetzung des Nachfeldes wird häufig eine kommunikativ-pragmatische Funktion zugeschrieben derart, dass ins Nachfeld besonders umfangreiche, semantisch gewichtige oder rhematische Satzglieder rücken“ (Eisenberg 2006: 401, so auch IDS-Grammatik 1997: 1478 und Günthner 2009: 35). Meiner Meinung nach stimmt hier vor allem die oben erwähnte Rhematizität, die auch in anderen, ähnlich funktionierenden Strukturen anzunehmen ist und auch angenommen wird (Zifonun 2001: 125), vgl. etwa das so genannte Vorfeld-es (Eisenberg 2006: 177, für die Parallele s. ebd., 329):

(59) Es zog mit mihr von Oberehn hinweck ein Man, so alda in den Weinreben $21 / 2 \mathrm{Jahr}$ gearbeidtet hatt (41r) $(\mathrm{G})$

(60) es wurde von da bericht es half aber nichts, armer du must leiden. (14) (J)

\footnotetext{
${ }^{61}$ Im Falle des Korrelat-es ist mit Subjekt- und Objekt-es gemeint, dass Subjekt- und Objektsätze korreliert werden.
} 
Dass aber etwas semantisch gewichtig, umfangreich oder „schwer“ (IDS-Grammatik 1997: 1479) ist, bedeutet noch nicht, dass es im Nachfeld steht, vgl.:

Die schrittweise Eroberung der Burg durch sehr tapfere Ritter im ersten Jahrhundert v.Ch. war ein wichtiges Ereignis.

Die Verschiebung des korrelierten Nebensatzes ins Nachfeld wird oft Extraposition genannt (s. etwa Duden-Grammatik 2005: 901). Dieser Begriff geht auf die Transformationsgrammatik zurück und hängt eng mit Ausklammerung zusammen. Beide bezeichnen die Wegbewegung des Nebensatzes aus dem Feld vor der rechten Klammer. Das Problem mit dieser Auffassung ist, dass das Mittelfeld für Komplementsätze gar nicht vorgesehen ist, weshalb eine Ausklammerung von ihm aus nicht möglich ist (Eisenberg 2006: 401). Stattdessen kann man davon ausgehen (ebd., 402, auch 327), dass diese Position bzw. diese Art von Nachfeldbesetzung bei Komplementsätzen und IKs grammatikalisiert ist. Auch Günthner (2009: 35) betont, dass der Extrapositionsgedanke zumindest für gesprochene Sprache nicht haltbar ist, da dort das Korrelierte recht umfangreich und inhaltlich komplex sein kann, sodass eine Rückführung auf eine Originalposition im Mittelfeld unwahrscheinlich ist.

Die bisher erwähnten Eigenschaften der es-Korrelate sprechen m.E. dafür, diese von solchen abzugrenzen, die durch Präpositionaladverbien ausgedrückt werden. Unterstützt wird diese Position auch durch die Existenz so genannter lexikalisierter Korrelate des Typs es scheint, es gilt, es heißt (teilweise auch es ist so), die es in ähnlicher Form bei anderen Korrelaten nicht gibt.

Schließlich gilt es auf die so genannten ergänzenden wenn-Sätze einzugehen (Eisenberg 2006: 344, Askedal 1990: 217). Zur Darstellung werden folgende Belege angeführt:

(61) Ich thue jedoch gerne ein Vergnügen umsonst, es freut mich, wenn sich ein anderer freut. (8) $(\mathrm{K})$

(62) Den ganzen Tag von früh bis abends musste ich streng arbeiten, so dass ich es für eine Ruh empfinde, wenn [ich] zum Gänsehüten geschickt werde. (16) (SCHG)

(63) Und es dauert noch lange bis Du bei Deiner Lina bist. (1915) (LB) 
(64) wir wohnten zwar untter einem dache | allein es währte doch ein zeitlang | Ehe wir uns sahen (1) (DM)

Wie die Belege zeigen, kommen als Junktor nicht nur wenn, sondern auch bis/ehe vor (zu als s. Zitterbart 2002a: 62 und IDS-Grammatik 1997: 1458, 1460). Solche wenn-Sätze werden gewöhnlich mit dass-Sätzen in Komplementfunktion in Zusammenhang gebracht. Unter semantischem Gesichtspunkt gilt im Allgemeinen, dass wenn-Sätze, wenn sie als Komplementsätze gebraucht werden sollen, dort stehen, wo auch dass-Sätze vorkommen können (Eisenberg 2006: 345). Im Gegensatz zu temporalen wenn-Sätzen, die durch Wann? erfragt werden können, stellen sie Antworten auf Was-Fragen dar (Cortès 2003: 147). Als Bedingung wird meistens die Faktizität des entsprechenden Prädikatsausdrucks erwähnt (ebd., s. auch IDS-Grammatik 1997: 1458f.). Was als und wenn angeht, so fügt die DudenGrammatik (2005: 1055) hinzu, dass „die Version mit als den Verlaufscharakter besonders zum Ausdruck [bringt]“ und „Entsprechende wenn-Nebensätze betonen, dass noch völlig offen ist, ob der ausgedrückte Sachverhalt eintritt oder nicht.“ (ebd., 1056) Das Vorkommen von bis und ehe (vgl. (63) und (64)) ist in meinem Korpus auf Verben des Dauerns beschränkt, was mit der Semantik des Junktors zusammenhängt. Grammatisch interessiert vor allem, dass mit wenn-Sätzen Formate vorliegen, die grundsätzlich für Adverbialfunktionen vorgesehen sind (Eisenberg 2006: 344). Deshalb ist das Vorhandensein eines Korrelates von zentraler Bedeutung. Es trägt nämlich dazu bei, aus dem Adverbial ein Komplement zu machen. Genau daraus leitet Eisenberg (ebd., 345, so auch 330) den Zwang zum Korrelat ab: „Der wenn-Satz ist an sich kein Ergänzungssatz. Er kann nur mithilfe eines geeigneten syntaktischen Kopfes in die Komplementposition gebracht werden.“ Absolut zwingend ist der Korrelatgebrauch zwar nicht (Cortès 2003: 147, 151), aber die Stellungsregularitäten, die von denen bei dass-Sätzen abweichen, scheinen für Eisenbergs Ansicht zu sprechen:

(61') Ich thue jedoch gerne ein Vergnügen umsonst. Wenn sich ein anderer freut, freut es mich

(62') wenn [ich] zum Gänsehüten geschickt werde, empfinde ich es für eine Ruh.

Wie aus den abgeänderten Beispielen ersichtlich, bleibt das Korrelat bei Voranstellung des wenn-Satzes erhalten, was für dass-Sätze nicht gilt. Dies ist ein Zeichen dafür, dass zur 
Etablierung eines wenn-Satzes in Komplementfunktion ein Korrelat im Hauptsatz auch dann nötig ist, wenn es bei dass-Sätzen sonst entfallen würde.

\subsubsection{Subordination, Integration und das Korrelat-es}

Im Folgenden soll gezeigt werden, dass es sinnvoll ist, das Korrelat-es unter Berücksichtigung seiner textgrammatischen Verwandten $\mathrm{zu}$ beschreiben. Es soll im Mittelpunkt stehen, wie syntaktisch gebundene Phorik entsteht. Dies soll hier als Syntaktisierung pro-propositionaler Phorik begriffen werden: Der Bezug auf Elementarsätze wird in das Format eines Satzgefüges „gedrängt“ („KM-interne Phorik“) und findet innerhalb dieses statt. Wie zu zeigen sein wird, ist dabei nicht von einer Dichotomie (entweder gebunden oder nicht-gebunden), sondern von mehreren Stufen auszugehen. ${ }^{62}$ Zur Darstellung wird auf den Begriff Integration zurückgegriffen, der in Anlehnung an Lehmann (1988) und Ágel/Diegelmann (2010) anhand dreier Parameter gedeutet wird:

- Position des NS

- Position des Korrelates im HS

- Form des Nebensatzes

Die ersten zwei Parameter stellen insofern eine allgemeinere Extension des Begriffes Integration dar, als es dabei darum geht, KVen innerhalb der Subordination zu verorten. Beim dritten Parameter handelt es sich hingegen nur um einen Teil der KV. Bevor mit der Erläuterung dieser drei Parameter angefangen wird, soll der Ausgangspunkt, d.h. die gemeinsame Behandlung nicht-gebundener und gebundener Phorik kurz begründet werden.

Durch das Korrelat-es kann auf die gleichen Inhalte und Formen Bezug genommen werden, die auch bei nicht-gebundener pro-propositionaler Phorik vorkommen. In der IDSGrammatik (1997: 1489) heißt es:

${ }_{62}$ Auch Ulvestad/Bergenholtz betonen (1983: 5f.), dass Strukturen, die nicht aus Haupt- und Nebensatz bestehen, in die Analyse mit einbezogen werden müssen. 
Mit dem Korrelat kann nämlich auf ebendie (latenten) Sachverhalte referentiell Bezug genommen werden, auf die auch mit der IK oder dem Satz Bezug genommen werden kann (unter der Bedingung der Referenzidentität). Insofern entspricht das Vorkommen als Korrelat den entsprechenden Vorkommen als KM-externe Anapher, Anadeixis.

Dieser Logik folgend kann man einen Beleg wie

(65) Sprecht eure Sprachen rein und klar, und erhaltet sie. Es ist wichtig. (24) (SB)

mit seinem Korrelat-Paar vergleichen:

(65')Es ist wichtig, dass ihr eure Sprachen rein und klar sprecht.

In (65') wird der Bezugselementarsatz in einen Hauptsatz eingebunden. Es entsteht ein Satzgefüge, in dem der Elementarsatz als abhängiger Subjektsatz erscheint. Dabei ist die Verweisrichtung im Falle des Korrelates kataphorisch. Dies ist ein wichtiger Unterschied, denn, wie in 2.3.1 argumentiert wurde, Kataphern können nicht als einfache Umkehrungen von Anaphern gesehen werden. Formal handelt es sich dabei um Elementarsätze, deren genaue Form beim Korrelat jedoch anders ist, als es bei nicht-gebundener Phorik der Fall war. Gebundenheit bedeutet hier semantische und syntaktische Abhängigkeit des dassSatzes vom Hauptsatz. Des Weiteren unterscheiden sich das anaphorische Beispiel und sein Korrelat-Paar darin, dass bei der Anapher die Proposition zuerst unabhängig assertiert wird. Erst in einem zweiten Schritt wird sie durch es wiederaufgenommen und so als abstraktes Objekt mit dem Prädikatsausdruck ist wichtig verbunden. Im Falle des Korrelates wird die Proposition eure Sprache rein und klar sprechen jedoch nicht unabhängig assertiert, sondern gleich bei der Äußerung gebunden (Zifonun 2001: 76).

Ein Vergleich ist weiterhin mit nicht-gebundenen kataphorischen Verweisen auf Propositionen vorstellbar, vgl.:

(66) Die Frau hatte Erbarmen mit mir und sagte zu mir, so dass es niemand hörte sonst: «Geh ins Bett und schlaf, ich rufe Dich dann, bleib solang.> (15) (SCHG)

Askedal bringt solche Beispiele mit wenn-Sätzen in Zusammenhang. Nach seiner Meinung besteht der Unterschied zwischen korrelathaltigen Bezügen und solchen ohne Korrelat in 
der „Spezifiziertheit der morphosyntaktischen Umgebung (wenn/als-Satz als Bezugsgröße), die man als eine Art Konstruktionsbedingtheit bzw. Grammatikalisierung auffassen kann.“ (1990: 217) Offensichtlich gilt das nicht nur für wenn-Sätze, sondern auch für dass-Sätze. In beiden Fällen geht es nämlich darum, dass Bezugnahmen innerhalb einer gebundenen Struktur stattfinden. Damit soll nicht gesagt werden, dass wenn-Sätze mit dass-Sätzen gleichzusetzen wären, vgl. weiter oben.

Vereinfacht kann man davon ausgehen, dass bei gebundener Phorik im Gegensatz zur nicht-gebundenen Unterordnung vorliegt. Dabei ist dieser Typ von Subordination ein besonderer. Zitterbart (2002: 14, 20, 33) spricht von „korrelativer Subordination“. Im Folgenden werden die oben angeführten drei Parameter auf KVen bezogen, um zu zeigen, dass die (syntaktische) Gebundenheit bzw. Bindung kataphorischen Verweisens nicht dichotomisch, sondern entlang Stufen erfasst werden muss. Unter a) wird auf die ersten beiden, unter b) auf den dritten Parameter eingegangen.

zu a)

Subordination ist eine der möglichen Formen von clause linkage (Lehmann 1988: 182). ${ }^{63}$ Lehmann nimmt Unterordnung an, wenn zwei Einheiten, die miteinander in Verbindung gesetzt werden, eine endozentrische Konstruktion formen (ebd.), in der eine der beiden Einheiten Kopf ist. Dabei ist die Relation zwischen diesen Einheiten entweder dependenziell oder „sociative“ (ebd., 183). Letztere Bezeichnung ist im Grunde genommen das Gegenteil von Dependenz, bei der wesentliche grammatische Eigenschaften der dependenten Einheit von der regierenden bestimmt werden. „Sociative“ sind bspw. Koordinationen und Appositionen. Aus Lehmanns Überlegungen geht hervor, dass das Konzept der Subordination skalar aufgebaut ist: Es wird ein Kontinuum angenommen. So sind bspw. die Relationen Dependenz und Sociation zwei Pole eines Kontinuums mit mehreren Stufen zwischen ihnen. Genau so muss man auch bei Subordination verfahren, bei der ebenfalls mit Abstufungen zu rechnen ist. Der Weg von Sociation zu Dependenz kann als Integration bezeichnet werden (ebd., 183):

\footnotetext{
63 „In the application of the term clause linkage, we will assume a broad concept of the clause which comprises any syntagm containing one predication. Syntactically, this means that - apart from nominal clauses - the uppermost controller of dependency in the syntagm is a verbal form.“ (1988: 182) Die Verbzentriertheit dieser Begriffsbestimmung ermöglicht es, statt clause Elementarsatz zu gebrauchen.
} 
The requirement in our definition that a subordinate construction must be part of a higher construction leaves large room for variation. For one thing, the subordinate syntagm may bear a sociative or a dependent relation to the main clause. For another, it may be subordinate to the main clause as a whole or rather to some constituent of the main clause. Neither of these alternatives is clear-cut; the differences are gradual. The two aspects taken together identify what I will call the integration of the subordinate construction into the main clause.

Während der Integration verliert der einzubindende Elementarsatz an Autonomie. Als Startpunkt kann dabei Parataxe gelten, den Endpunkt bildet die so genannte Einbettung (,embedding“), bei der ein Elementarsatz $\mathrm{zu}$ einem nicht-satzförmigen Satzglied „herabgedrückt" wird, d.h. zu einer Konstituente innerhalb des superordinierten Satzes wird (ebd., 184), vgl. etwa den Kontrast zwischen

Als Peter über die Straße lief, hat er die Tram nicht beachtet.

vs.

Über die Straße laufend hat Peter die Tram nicht beachtet.

Wichtig ist für die Darstellung hier, dass korrelative Subordinationen auf halbem Weg zwischen Parataxe und Hypotaxe verortet werden (ebd., 185, 189, 218). Eine präzisere Bestimmung ist allerdings notwendig, weil, wie dies auch Lehmanns Beispielen zu entnehmen ist, in der Mitte vieles anzusiedeln ist. $\mathrm{Zu}$ diesem Zweck wird auf die Theorie der expliziten Junktion von Ágel/Diegelmann (2010) zurückgegriffen. Sie thematisieren zwar Junktion durch Inhaltsrelationen und nicht Einbettungen wie Subjekt- und Objektsätze im Sinne der Satzsemantik von Peter von Polenz (1985). Eine (wenn auch nicht komplette) Anwendung dieser Theorie auf es-Sätze scheint mir dennoch möglich, da Ágel/Diegelmann (2010: 349) Junktion grundsätzlich syntaktisch erfassen: „Der Punkt ist, dass Junktion ein syntaktisches Konzept im Dienste der Semantik ist.“ Ihre Kriterien, deren Anwendung innerhalb der Junktionsklassen zu verschiedenen Junktionstechniken führt, sind ebenfalls syntaktischer bzw. teilweise morphosyntaktischer Natur (ebd., 357, 360f. und 365f.). Zentrale Begriffe dieser Theorie sind Aggregation und Integration. Mit Bezug auf die 
Verbindung von zwei syntaktischen Strukturen beziehen sie sich auf die syntaktische Kohäsion zwischen jungierten Einheiten und auf den Beitrag der Syntax zur semantischen Interpretation (ebd., 349). Vereinfacht lässt sich sagen, dass Aggregativität bei geringer syntaktischer Kohäsion und einem geringen Beitrag syntaktischer Markierung zur Semantik vorliegt, während für Integrativität das Umgekehrte zutrifft. Dabei bilden sie die beiden Pole eines Kontinuums und lassen sich nicht nur auf einzelne Techniken der Subordination anwenden, sondern auch auf die Form des NS und dessen Einbindung in den Hauptsatz, vgl. b) weiter unten. Korrelate werden in Ágel/Diegelmann (2010: 365f.) in dem Kapitel zur Subordination behandelt. Auch korrelative Verbindungen durch es sind subordinierend, weil es sich dabei um regierte Konnekte, ${ }^{64}$ nämlich um Subjekt- oder Objektkomplemente, handelt. Regiertheit ist eines der beiden wichtigsten Kriterien für Unterordnung in der Theorie der expliziten Junktion in Ágel/Diegelmann (2010: 357). Das andere entscheidende Kriterium ist, dass bei Subordination verbale Konnekte regiert werden, nicht etwa nominale (was für Inkorporation sprechen würde, vgl. „embedding“ in Lehmann 1988). Was Regiertheit angeht, so muss betont werden, dass im Falle der es-Korrelate der jeweilige Komplementsatz vom Prädikatsausdruck regiert wird, während Ágel/Diegelmann (2010) Supplementsätze untersuchen, bei denen das entsprechende Konnekt (der NS) vom Junktor regiert wird. Was KVen anbelangt, gibt es zwischen Korrelaten bei Komplement- und Supplementsätzen wichtige Unterschiede:

Platzhalter für Komplemente verweisen typischerweise aus dem Mittelfeld vor auf einen Satz oder eine IK im Nachfeld. Verweis findet also innerhalb der eigentlichen Feldstruktur statt. Platzhalter für Supplemente verweisen aus der Vorfeldstellung zurück auf das linke Außenfeld. (IDS-Grammatik 1997: 1492)

Den ersten Fall repräsentieren es-Korrelate im Mittelfeld des HS, während für Supplementkorrelate etwa folgendes Beispiel angeführt werden kann:

Wenn du nach Hause gehst, dann gehe ich mit. ${ }^{65}$

Obwohl KVen bei Supplementen und Komplementen nicht gleichgesetzt werden können, halte ich die Anwendung der Ágel/Diegelmann'sche Theorie, wie oben erwähnt, für

\footnotetext{
${ }^{64}$ Unter Konnekte sind allgemein zu jungierende oder jungierte Strukturen zu verstehen.
}

${ }^{65} \mathrm{~S}$. dazu weiter unten. 
möglich, weil der Bestimmung der Integrativität von Konnekten hauptsächlich syntaktische Kriterien zugrundegelegt werden. Im Falle der Subordination sind es (s. die Parameter oben):

- die Position des Korrelates im HS und

- die Position des NS in der Felderstruktur

Die Aufgabe besteht nun darin, Junktion durch KVen innerhalb der Subordination zu verorten, indem bestimmt wird, wie integrativ diese Technik im Vergleich zu anderen Subordinationstechniken ist. Was Korrelate angeht, so werden resumptive und nichtresumptive unterschieden (Ágel/Diegelmann 2010: 365f.):

Ich fahre nach Hause dazu, um meine Familie zu sehen.

Ich fahre dazu nach Hause, um meine Familie zu sehen.

Für resumptive (erstes Beispiel) ist typisch, dass sie die letzte Stelle im Hauptsatz besetzen, wenn der NS nachgestellt ist. Sie markieren Kontaktstellung von Korrelat und NS: Korrelat und NS gehören demselben Stellungsfeld an (IDS-Grammatik 1997: 1477). Diese Stellung ist bei es (s. 2.3.2) nicht möglich. Nichtresumptive Korrelate (zweites Beispiel) besetzen das Mittelfeld des HS (Ágel/Diegelmann 2010: 366). Resumptive kommen auch bei vorangestellten NS vor (ebd.):

Um meine Familie zu sehen, dazu fahre ich nach Hause. ${ }^{66}$

In meinem Korpus kommt es in dieser Position nicht vor. Sie ist aber möglich, vgl. das Beispiel Dass/ob/wie du nach Berlin fährst, es interessiert ihn in Eisenberg (2006: 329). Möglich ist des Weiteren ein es im Mittelfeld im nachgestellten HS, vgl. Dass/ob/wie du nach Berlin fährst, er weiß es (ebd., s. auch IDS-Grammatik 1997: 1479). Beide Vorkommen sind nach Eisenberg resumptiv. Sie sind m.E. besonders aggregativ. Im ersten Fall steht das es im Vorfeld des HS und verweist auf den NS im linken Außenfeld. Im zweiten Fall steht es im Mittelfeld des HS. In beiden Beispielen finden wir im HS Verbzweitstellung, die bei Einbettungen als aggregativ eingestuft werden kann. Integrativ

\footnotetext{
${ }^{66}$ Siehe auch das Beispiel mit dann oben.
} 
wäre nämlich Verberststellung, bei der das es auch wegfallen müsste. In Anlehnung an die Bestimmung des Integrationsgrades von resumptiven und nichtresumptiven korrelativen bzw. nicht-korrelativen Junktionen bei Ágel/Diegelmann (2010: 365f.) ergeben sich somit beim es in einem ersten Schritt nur zwei Optionen, da Resumptivität in meinem Korpus nicht vertreten ist. Entweder steht das Korrelat im Mittelfeld oder es wird gar kein Korrelat gesetzt:

(67) Könnte es nicht sein, daß also eine inflationäre Bewegung des Geldes eine natürliche oder eine notwendige Bewegung innerhalb des Wirtschaftssystems ist? (6) (SB)

(68) Aber diese Bezeichnungen machen es ja eigentlich deutlich, wie man zu diesen Leuten steht (13) (SB)

bzw.

(67') Könnte nicht sein, daß also eine inflationäre Bewegung des Geldes eine natürliche oder eine notwendige Bewegung innerhalb des Wirtschaftssystems ist?

(68') Aber diese Bezeichnungen machen ja eigentlich deutlich, wie man zu diesen Leuten steht.

Als integrativer gelten unter diesen Belegen die Fälle mit Mittelfeld-Korrelat, weil „das nichtresumptive Korrelat den Anschluss des internen Konnekt [des regierten NS, D.C.] nicht nur ermöglicht [...], sondern auch antizipieren lässt.“ (ebd., 366) Die Frage ist nun, wie mit subjektischen Vorfeld-Korrelaten, die Ágel/Diegelmann (2010) nicht thematisieren, umgegangen werden kann, vgl::

(69) Es war eben klar, daß die östlichen Medien zum Beispiel eine Angelegenheit wie den Einmarsch der Verbündeten in die Tschechoslowakei [...] eindeutig nach einer vorgegebenen Regelung formulierten (8) (SB)

Das Korrelat in (69) ist, ähnlich wie die im Mittelfeld, klar nicht resumptiv. Für die Integrativität des NS sprechen die antizipatorische Kraft des Korrelates und die Tatsache, dass es den direkten Anschluss des NS an den HS nicht verhindert. Diese Eigenschaften legen eine Behandlung dieses Falles nahe, wie sie bei Mittelfeld-Korrelaten vorgenommen 
wurde. Allerdings macht Zitterbart (2002a: 58ff.) darauf aufmerksam, dass beim Korrelat-es im Vorfeld - im Gegensatz zu dem im Mittelfeld - syntaktische Ambivalenz vorliege, weil es unter syntaktischem Aspekt auch als ,reines“ Vorfeld-es ohne Korrelatfunktion (Typ: Es wird ein Beleg gedruckt) gedeutet werden kann. Auch die Umstellprobe im HS (Klar war eben, daß [...]), die zum Wegfall des es führen kann, deute auf diese Ambivalenz hin, weil bei einer Umstellung auch das Vorfeld-es verschwinde (Ein Beleg wird gedruckt). Obwohl ein Hinweis auf Ähnlichkeiten zwischen Korrelat- und Vorfeld-es bspw. mit Bezug auf die bei beiden vorhandene Rhematisierungsfunktion gut begründet werden kann (vgl. Zifonun 2001: 125 und 2.3.2), stellt sich beim genaueren Hinsehen heraus, dass sich die Annahme syntaktischer Mehrdeutigkeit als problematisch erweist, und zwar aus zwei Gründen. Einerseits muss das Vorfeld-es entfallen, wenn das Vorfeld anderswie besetzt wird, während das Korrelat fehlen, aber auch stehen bleiben kann, wie Zitterbart (ebd., 59) selbst zeigt. Andererseits scheint mir Ambivalenz eher zwischen Korrelat-es und Rechtsversetzungen vorzuliegen. Diese Mehrdeutigkeit betrifft dabei eher die hörer- bzw. leserseitigen Erwartungen, die sich nach Realisierung der gegebenen syntaktischen Struktur ergeben, vgl.:

Es war eben klar, daß [...]

vs.

Es war eben klar, das Problem, von dem die Rede war.

Diese Beispiele zeigen m.E., dass die Annahme von Mehrdeutigkeit beim (Subjekts)Korrelat-es und dem (herkömmlichen) Vorfeld-es deshalb nicht stimmt, weil nach der Realisierung von Es war eben klar nur noch eine rechtsversetzte NP außerhalb, nicht jedoch eine innerhalb der Felderstruktur zu erwarten ist (Es war das Problem eben klar ist nach der Realisierung von Es war eben klar nicht mehr möglich). M.a.W.: Bezüglich der hörer- bzw. leserseitigen Erwartungen (vgl. hierzu auch Fußnote 58) sind nur die beiden, oben angeführten Fortsetzungsmöglichkeiten gegeben. Nimmt man nun eine Ambivalenz in diesem Sinne an, so muss man einsehen, dass sie auch für das Mittelfeld-es gilt, vgl. etwa:

(70) Aber diese Bezeichnungen machen es ja eigentlich deutlich, wie man zu diesen Leuten steht (13) (SB) 
(70’) Aber diese Bezeichnungen machen es ja eigentlich deutlich, unsere Stellung zu diesen Leuten

Die Ambivalenzthese von Zitterbart kann also nicht dazu führen, Vor- und Mittelfeld-es unterschiedlich zu behandeln. Des Weiteren habe ich in 2.3.2 dafür argumentiert, dass Subjektsätze korrelierende $e s$-Vorkommen von solchen, die Objektsätze binden, funktional nicht getrennt werden sollten. Hinsichtlich der Integrativität ist eine Differenzierung zwischen Vorfeld- und Mittelfeldvorkommen trotzdem möglich. Man kann argumentieren, dass das eventuelle Wegfallen des Korrelat-es im Vorfeld anderen Gesetzmäßigkeiten unterliegt als das des Mittelfeld-es. Das Verschwinden des Korrelates vom Satzanfang (War eben klar, daß [...]) ist hauptsächlich im gesprochenen Deutsch möglich, weil es dort als Platzhalter semanto-pragmatisch vielfach überflüssig ist (Auer 1993: 197). Setzung und Nicht-Setzung des es im Mittelfeld werden hingegen durch andere Faktoren gesteuert, s. 2.3.2. Beim Subjektskorrelat-es ist die Vorfeldposition immer verfügbar. Wird jedoch ein es im Mittelfeld gesetzt, so kann dies als ein besonderes Signal für Integration betrachtet werden. ${ }^{67}$ Auf der Basis der bisherigen Überlegungen und der Ausführungen in Ágel/Diegelmann (2010: 365f.) können somit folgende Stufen der Integration beim Korrelat-es angenommen werden:

1. NS im Nachfeld, kein Korrelat im HS

2. NS im Nachfeld, Korrelat im Vorfeld des HS (nur Subjektskorrelate)

3. NS im Nachfeld, Korrelat im Mittelfeld des HS (Subjekts- und Objektskorrelate)

4. NS im Vorfeld, kein Korrelat im HS (Einbettung)

Die Integration nimmt zwischen Stufe 1 und 4 zu. Den Stufen 2 und 3 ist zu entnehmen, dass bei Integrativität nicht die syntaktischen Funktionen Subjekt und Objekt zählen, sondern die Stellung des Korrelates. Bei Stufe 3 ist anzumerken, dass die Setzung des Korrelates im Mittelfeld bei bestimmten Verben und Konstruktionen obligatorisch werden

${ }^{67}$ Möglich ist auch die Annahme einer schwächeren Integration bei korrelathaltigen Verbindungen, wenn davon ausgegangen wird, dass bei Weglassung des Nebensatzes kein Hauptsatzfragment, sondern ein grammatisch vollständiger Hauptsatz entsteht, vgl. dazu IDS-Grammatik (1997: 2251). 
kann, vgl. Zitterbart (2002a: 84, 96). Den Endpunkt einer solchen obligatorischen Setzung bilden lexikalisierte es-Korrelate wie in es gilt/heißt, die auch dann nicht wegfallen, wenn der NS im Vorfeld steht. Dies könnte Stufe 5 sein, aber da die Lexikalisierung des Korrelates in diesen Fällen mit Bedeutungsveränderungen einhergeht (s. Zitterbart 2002a: 81f., ähnlich zu es scheint ebd. und Eisenberg 2006: 364f.), werden diese nicht mehr als klassische Korrelate angesehen. Wichtig ist auch, dass die Stufen 2-3 enger miteinander verbunden sind, da in ihnen Korrelatsetzung vorliegt. Sie sind insgesamt weniger integrativ als Einbettungen (Stufe 4). Entsprechend dieser Trennung wird in Anlehnung an Zitterbart (2002a: 20) im Falle korrelierter NS im Nachfeld von ihrem Anschluss an den Hauptsatz gesprochen, nicht aber von ihrer Einbettung. Damit im Zusammenhang ist noch der Frage nachzugehen, ob und wie sich wenn-Sätze in das obige Schema fügen. Relevant ist diese Fragestellung, weil für ergänzende wenn-Sätze typisch ist, dass bei ihrer Voranstellung das Korrelat-es im HS nicht verschwindet:

(71) Weiter wenn Du, mein Jakob, mir wieder Javakaffee von derselben Güte zu dem gleichen Preis wie den vorigen $1 / 2$ Zentner besorgen könntest, wärs recht. (17) (L)

Wie Eisenberg (2006: 345) erläutert, kann dieses es nicht wegfallen, weil es den Komplementstatus einer genuin adverbialen Satzform $\mathrm{zu}$ markieren hat. Im Ágel/Diegelmann'schen System (2010: 365) entspräche dieser Typ dem integrativsten mit vorangestelltem NS und Korrelat im Mittelfeld des nachgestellten HS wie in

Um meine Familie zu sehen, fahre ich dazu nach Hause. (ebd., 366).

Allerdings ist die Anbindung des NS an den HS bei ergänzenden wenn-Sätzen nicht deshalb stark, weil der NS vorangestellt ist und im nachgestellten HS ein Korrelat steht, sondern weil diese Bindung aus einem syntaktischen „Zwang“ zur Kennzeichnung eines Komplementes resultiert. Das heißt, in diesem Fall interessieren nicht die Position des regierten Konnektes und die des Korrelates, sondern die Ausweisung des wenn-Satzes als Komplement. Das es-Korrelat verhält sich zu seinem NS auch semantisch anders als es dazu tut. Denn dazu ist semantisch mit dem Subjunktor um und der finalen Inhaltsrelation in Verbindung zu setzen, während eine solche Beziehung zwischen es und wenn (und der konditionalen Inhaltsrelation) nicht hergestellt werden kann. Dies wiederum ist aus dem 
Komplementstatus abzuleiten, an den keine solchen Inhaltsrelationen gebunden sind. M.a.W.: Die Korrelate, die in Ágel/Diegelmann (2010) angeführt werden (dazu), markieren Inhaltsrelationen, das es den Komplementstatus. Für den Komplementstatus ergänzender wenn-Sätze spricht im Übrigen auch, dass vorangestellten Adverbialsätzen vorrangig resumptive Korrelate folgen, s. IDS-Grammatik (1997: 1492f.). Wenn-Sätze werden also durch es auf die integrativste Weise korreliert, was jedoch nicht aus der Korrelat-, sondern aus der (Komplement)Anzeigefunktion folgt.

Abschließend soll noch ein wichtiges kommunikativ-semantisches Merkmal von KVen erörtert werden, das die betroffenen HS gekennzeichnet. Bei Einbettungen und nachgestellten Komplementsätzen ohne Korrelat im HS kann man festhalten, dass die HS ungrammatisch werden, wenn die NS wegfallen. Das zeigt ihre starke Integration. Bei KVen liegen die Verhältnisse jedoch teilweise anders, weil Hauptsätze mit Korrelat ihre Grammatikalität rein formal auch dann nicht einbüßen, wenn das Korrelierte wegfällt. Semantisch gilt dies allerdings nicht mehr (Zitterbart 2002a: 21), denn beim Wegfallen des Korrelierten muss das Korrelat eben anders interpretiert werden, nämlich anaphorisch und nicht mehr als (per definitionem kataphorisches) Korrelat, vgl.:

(72) Es ist auch uff den Sontag nach Jacobi ahngestellet worden im Land, alle Tag gegen Abend zu betten funf Vatterunser und funf Ave Maria und einen Glauben (31) (BL)

VS.

(72')Es ist auch uff den Sontag nach Jacobi ahngestellet worden im Land.

Im zweiten Fall bliebe meiner Meinung nach keine andere Deutungsmöglichkeit als der Versuch, den phorischen Inhalt von es im Vortext zu suchen. Aus diesem Umstand folgt, dass

der Hauptsatz, wenn das Korrelierte verschwindet, streng genommen als Hauptsatzfragment betrachtet werden muss: „HAUPTSATZFRAGMENTE sind ein finites Verb enthaltende Teile einer komplexen KM, die bei der Ablösung aus der KM zwar keine selbständige KM darstellen, aber durch einen Untersatz zu einer KM ergänzt werden.“ (IDS-Grammatik 1997: 2238) Zitterbart (2002a: 47) argumentiert gegen diese Position: 
Man kann hier von einer „bipolaren kommunikativen Struktur“ sprechen: das Korrelat im Matrixsatz weist auf den Nebensatz hin, der Nebensatz erfüllt die Erwartung. Deswegen sind Matrixsätze von korrelierten Nebensätzen nicht als Matrixsatzreste oder -fragmente zu betrachten: Der Matrixsatz ist nur einer der beiden Pole einer einzigen Struktur. Die Verflechtung der beiden Strukturteile - Matrix- und Nebensatz - ergibt sich in allen Hinsichten.

Das alles ist m.E. jedoch kein Argument gegen den Fragmentstatus von Hauptsätzen, deren korrelierter Nebensatz abgelöst wird. Fragmentiertheit wird in der IDS-Grammatik auf kommunikative Aspekte bezogen: Sie bedeutet, dass Hauptsatzfragmente alleine keine KM bilden. Wenn also der Nebensatz die Erwartung erfüllt, so ist dies ein Argument für und nicht gegen die Unvollständigkeit. Dass der Matrixsatz (Hauptsatz) nur einen der Pole in KVen darstellt, spricht wiederum dafür, dass er nicht vollständig ist, da er erst in einer KV vollständig werden kann. Schließlich ist die formale Grammatikalität, die ich oben angedeutet habe, auch kein Problem für diese Art Bestimmung von „nebensatzlosen“ korrelathaltigen Hauptsätzen, wie Zitterbart (ebd., 12) dies sieht, denn Vollständigkeit/Fragmentiertheit werden in der IDS-Grammatik unter kommunikativem Gesichtspunkt betrachtet, vgl. dazu auch die Definition von KM (1997: 91), der in obiger Definition eine konstitutive Rolle zukommt. Die semantische Fragmentiertheit des Hauptsatzes ist ein Indiz für den besonderen Charakter der korrelativen Subordination. „Die Rollen von Matrix- und Nebensatz sind zwar klar verteilt, aber dennoch hängt nicht nur der Nebensatz vom Matrixsatz ab, sondern auch der Matrixsatz vom Nebensatz“, so Zitterbart (2002a: 46). Es handelt sich dabei um eine Art „Interdependenzverhältnis“ (ebd., 46f.). Dies soll zum Ausdruck bringen, dass das Korrelierte vom Prädikatsausdruck im Hauptsatz abhängt, weil es sein Komplement darstellt. Andererseits besteht aber auch insofern eine gewisse semantische Abhängigkeit des Hauptsatzes vom Nebensatz, als Ersterer kommunikativ nur zusammen mit Letzterem vollständig sein kann.

Haupt- und Nebensatz werden also bei KVen auf spezielle Art und Weise jungiert. Nicht zufällig werden Nebensätze, die einen Vertreter, d.h. ein Korrelat, im Hauptsatz haben, in der IDS-Grammatik (1997: 1488) in Form von KVen als eigene Konstruktionskategorie für Komplemente eingeführt. Soll also der Valenzrahmen etwa eines Verbs bestimmt werden, so ,ist die Belegbarkeit durch IK oder SJS (und seine Unterarten) für jede Komplementstelle eigens zu vermerken.“ Dabei gilt, dass diese Belegungen auch durch KVen vorgenommen werden können (ebd.). Zusammenfassend wird festgehalten, dass die 
korrelative Subordination unter semantischem Aspekt deshalb einen besonderen Typ von Subordination darstellt, weil der Nebensatz vom Hauptsatz zwar syntaktisch abhängt, dabei der Hauptsatz jedoch - weil fragmentiert - semantisch auf den Nebensatz angewiesen ist.

$z u b)$

Die Heranziehung des Integrationsaspekts kann auch dann fruchtbringend sein, wenn es um die Form des Nebensatzes geht. Folgende Parameter müssen dabei berücksichtigt werden:

- Subordinationsmarker: Subjunktor und Verbletztstellung

- Finitheit/Infinitheit (mit und ohne $z u$ )

Auch hier geht es - wie in a) - um das Zusammenspiel von Syntax und Semantik. Ágel (2007) spricht dabei von Statusheterologie und Statushomologie. Letztere bestimmt er (2007: 45) wie folgt:

Syntaktischer Superordination entspricht semantische Superordination, syntaktischer Subordination semantische Subordination. Mit anderen Worten, die subordinierten Diskursteile sind syntaktisch wie semantisch in die superordinierten integriert, d. h. ihre syntaktische und semantische Organisierung erfolgt von den superordinierten Diskursteilen aus. Mit Hilfe eines kunsthistorischen Importbegriffs lässt sich die Organisationsweise, die die genannten Beispieltypen verbindet, zentralperspektivisch bezeichnen. Ich möchte den Parameter, der für die zentralperspektivische Organisationsweise der Grammatik verantwortlich ist, den Integrationsparameter nennen.

Für Statusheterologie hingegen gelte (ebd.):

Mit anderen Worten, die Syntax und die Semantik sind hier von verschiedenen Perspektiven aus organisiert. Mit Hilfe ebenfalls eines kunsthistorischen Begriffs lässt sich die Organisationsweise, die die genannten Beispieltypen verbindet, aspektivisch bezeichnen. Ich möchte den Parameter, der für die aspektivische Organisationsweise der Grammatik verantwortlich ist, den Aggregationsparameter nennen. 
Im Mittelpunkt steht hier, wie die Markierung von Subordination im NS zu dessen Integration beiträgt bzw. wie fehlende Markierung von Subordination zur Aggregation führt. Zur Veranschaulichung sollen hier zwei Beispiele dienen (ebd., 44):

(i) Würden Sie so freundlich sein, mir zu helfen? (DUW 2003)

(ii) Würdest du so lieb sein und das für mich abgeben? (Sommerfeldt/Schreiber 1983: 321)

(i) ist ein Beispiel für die Realisierung von Adjektivvalenz in Form einer Infinitivkonstruktion mit $z u$, während Valenztheorien keine Adjektivvalenz mit undAnschluss wie in (ii) vorsehen (ebd.). Dabei liegt semantische Abhängigkeit nicht nur bei (i), sondern auch bei (ii) vor. Ein wesentlicher Unterschied ist jedoch zwischen (i) und (ii), dass bei (ii) keine syntaktische Abhängigkeit vorliegt, während in (i) die syntaktische Abhängigkeit durch den Infinitiv mit $z u$ markiert wird. Nichtsdestotrotz besteht eine strukturelle Gemeinsamkeit (würde-Form + so + Adjektiv + Infinitivanschluss) zwischen (i) und (ii). Um den (adjektiv)valenzbezogenen und strukturellen Parallelen Rechnung zu tragen, schlägt Ágel vor, solche Fälle so zu erklären, dass man aggregativere ((ii)) und integrativere ((i)) Realisierungen der Adjektivvalenz annimmt (ebd., 45). Analog zu den Adjektiv-Beispielen könnte man nun auch den Grimm-Beleg in Ulvestad/Bergenholtz (1983: 6) anführen:

Endlich wagte er es und trat heran.

vs.

Endlich wagte er es heranzutreten.

Bei wagte liegt im ersten Beispiel semantische, nicht aber syntaktische Subordination vor, während die Unterordnung im zweiten Beispiel durch die IK auch formal, d.h. syntaktisch zustande kommt. Belege aus meinem Korpus zeigen auch, dass es manchmal gar keine Nebensätze im herkömmlichen Sinn sind, auf die sich das es bezieht, vgl.: 
(73) Die 1. Großmutter in Chrasic ist immer kränklich, sie weiß noch nichts vom Tode der 1. Tante Fleischner. Ja, ich kann es auch nicht glauben, u. doch ist es wahr, sie kann den schönen Frühling, nicht mehr athmen. (4) (KOR)

In Anlehnung an den Problemaufriss in Hegedüs (2007: 260) kann man bei (73) die Frage stellen: Ist das es in diesem Beleg ein Korrelat? Hält man am Formalen fest, so muss man die Frage zunächst mit Nein beantworten, denn die Sequenz ,sie kann den schönen Frühling, nicht mehr athmen“ ist unter formalem Aspekt kein Nebensatz: Sie enthält keinen Subjunktor und weist keine Verbletztstellung auf. Andererseits liegt jedoch semantische Subordination vor, weil die KV für ein Komplement steht: Es ist ein satzförmiges Subjekt $\mathrm{zu}$ ist wahr und satzförmiges direktes Objekt zu glauben. Formal teilweise anders sieht es im folgenden Beleg aus:

(74) es were am allerbesten, wir blieben wie wir sein, ist doch nichts den hader und [1246] streit im dorffe gewesen (34) (NE)

Der Unterschied zwischen (74) und (73) besteht darin, dass in (74) das Verb im korrelierten Elementarsatz im Konjunktiv steht. Mit Auer (1998: 297ff.) möchte ich bei (73) von abhängigen Hauptsätzen (AHS), bei (74) von uneingeleiteten Nebensätzen (UNS) sprechen. In beiden fehlen „kanonische Subordinationsmarker“ (ebd., 285), nämlich Verbletztstellung und Subjunktor. Es gibt jedoch auch andere Nebensatzmerkmale wie den Konjunktiv, die Verschiebung der deiktischen Kategorien Person, Ort und Zeit und die Ersetzung des Imperativs durch Modalverben, von denen der Konjunktiv in (74) für die Annahme eines UNS spricht. Dieses (wenn auch einzige) Nebensatzmerkmal deutet darauf hin, dass (74) näher am Nebensatzpol steht als (73). Auf der Basis der bisher angeführten Beispiele kann man nun Stufen der Nebensatzhaftigkeit und daran gebunden Stufen der Korrelathaftigkeit annehmen.

Stufe 0: Anaphorik

Stufe 1: Kataphorik, semantische Abhängigkeit, AHS 
Stufe 2: Kataphorik, semantische und niedrige syntaktische Abhängigkeit: UNS

Stufe 3: Kataphorik, semantische und syntaktische Abhängigkeit: NS (Verbletzt, Subjunktor)

Von Korrelaten kann gesprochen werden, sobald Kataphorik und semantische Abhängigkeit feststellbar sind. Prototypisch sind jedoch Korrelate, bei denen semantische und syntaktische Abhängigkeit vorliegen bzw. miteinander korrelieren, wobei bei Letzterer die kanonischen Subordinationsmarker Verbletztstellung und Subjunktor vorhanden sind. Bei der schrittweisen Einbindung der Bezugsgröße in einen Nebensatzrahmen (Stufe 1-3) handelt es sich also um den schrittweisen formalen Ausbau semantischer Abhängigkeit bzw. die schrittweise Integration des NS in den HS.

Ergänzt werden muss dieses Bild noch um den Parameter Finitheit/Infinitheit, der ebenfalls die Form des NS betrifft. Möglich sind hier IKs ohne oder mit $z u$ und finite Nebensätze:

(75) Das ander aber, Lottern und Dachmachen, das verricht ich mit eygner Hand. Die Zeit wolt es nicht leiden, viel Leuth nemen, dan wie gehöret, wie es uns ergangen war, so konten wir nicht viel zum Besten haben (16) (BL)

(76) es ist eine Eigenart dieser Krankheit, sich nach wiederholtem Schneiden wieder zu bilden. (4) $(\mathrm{K})$

(77) es wäre zu wünschen, daß es ihm gut geht. (6) (K)

Im Allgemeinen gilt, dass infinite Formen integrativer sind, weil „loss of personal conjugation“ in Richtung Nominalisierung zeigt (Lehmann 1988: 200). Mit Ágel/Diegelmann (2010: 363) wird die IK ohne $z u$ (s. (75)) dennoch als die aggregativste Form angesehen, ,weil der Infinitiv ohne $z u$ normalerweise statusregiert ist und daher - als Teil eines Verbalkomplexes - keine ,neue' Sachverhaltsdarstellung indiziert.“ Ich würde noch hinzufügen, dass solche IKs an nominale Rechtsversetzungen erinnern, die an ihren Linkskontext auch aggregativ angeschlossen werden. Finite Formen (etwa der $d a \beta$-Satz in 
(77)) befinden sich dann zwischen $z u$-haltigen IKs (vgl. (76)) und solchen ohne $z u$. Dieser Differenzierung kann Rechnung getragen werden, indem IKs ohne $z u$ zwischen Stufe 0 und 1 unterkommen, während durch IKs mit $z u$ eine neue Stufe, nämlich Stufe 4 etabliert wird.

Führt man nun die Parameter und Stufen in a) mit denen in b) zusammen, so könnte das wie folgt aussehen:

Stufe 0: Anaphorik

Stufe 1: Kataphorik, semantische Abhängigkeit: Inf. ohne $z u$

Korrelat im Vorfeld

Korrelat im Mittefeld

Stufe 2: Kataphorik, semantische Abhängigkeit: AHS

Korrelat im Vorfeld

Korrelat im Mittefeld

Stufe 3: Kataphorik, semantische und niedrige syntaktische Abhängigkeit: UNS

Korrelat im Vorfeld

Korrelat im Mittefeld

Stufe 4: Kataphorik, semantische und syntaktische Abhängigkeit: NS (Verbletzt, Subjunktor)

Korrelat im Vorfeld

Korrelat im Mittefeld

Stufe 5: Kataphorik, semantische und syntaktische Abhängigkeit: Inf. mit $z u$

Korrelat im Vorfeld

Korrelat im Mittefeld

Stufe 6: Einbettung mit Korrelat im (nachgestellten) HS: vorangestellte wenn-Sätze 
Zum Schluss soll auf ein Spezifikum des es eingegangen werden, dem in der Fachliteratur wenig Aufmerksamkeit geschenkt wird. Dieses Merkmal betrifft dabei zahlreiche Belege in meinem Korpus. Es ist relevant, weil es die kataphorische korrelative Bindung schwächt. Es handelt sich dabei um Belege, in denen die Verweisrichtung nicht klar bestimmt werden kann:

(78) Ich weiß allerdings nicht mehr, ob ich denn, wie ichs euch mittheilte, gar nichts auch nur andeutungsweis beifügte, daß die Sach für jetzt noch nicht an die große Glocke gehört (17) (L)

(79) Als ich nun heim kam, so schickt mir der Herr Magister gleich einen boten, und sagte recht zänckisch zu mir, seit ihr den schon wider kommen, Ja, was ist den draus worden, ich kans ihm selber nicht sagen was draus werden wil. (28) (NE)

(78) und (79) stellen bezüglich der Verweisrichtung teilweise andere Probleme dar. In (78) hat man es mit einem Einschub zu tun, der das Korrelat-es beinhaltet. Den anderen Teil der KV liefert das Segment ob ich denn gar nichts auch nur [...]. Der ob-Satz ist also Teil der $\mathrm{KV}$, gleichzeitig stellt er das satzförmige direkte Objekt zu wissen dar. Streng genommen entstehen hier sowohl ein Rück- als auch ein Vorverweis. Semantisch gesehen muss man allerdings hinzufügen, dass das inhaltlich Wesentliche erst nach dem Einschub kommt. Die Phorik in diesem Beispiel ist jedoch auch auf einer anderen Ebene, und zwar der metasprachlichen, zu fassen. Der Einschub ist hier eine metakommunikative Äußerung. Darunter sind zu verstehen „Äußerungen also, die sich auf die Kommunikation selbst beziehen, oder genauer, auf eine Äußerung, die derselben Kommunikationssituation angehört wie die metakommunikative Äußerung.“ (Stein 1995: 212) Durch den Einschub und so auch durch es wird auf eine frühere Gesprächssituation verwiesen. Meiner Meinung nach liegt hier ein Vergleich mit wie gesagt/wie ich schon sagte/wie schon gesagt wurde auf der Hand. Stein (ebd., 243) schreibt diesen ,gesprächsspezifischen Formeln“ „äußerungskommentierende Metakommunikation“ als dominante Funktion zu. Ihr Funktionsspektrum umfasst jedoch auch „Herstellung von Textbezügen: Verweis auf Vorangegangenes, Bekräftigung des Gesagten, Gliederungssignal“ (ebd.). 
Teilweise anders sind die Verhältnisse in (79). Hegedüs (2007: 260) spricht in diesem Zusammenhang von „Korrelate[n] in Mittelposition“:

Sie werden anscheinend originär als rückverweisende Elemente eingesetzt, was man daran sehen kann, dass der angeschlossene Nebensatz einen ähnlichen (oder leicht präzisierten) Inhalt hat wie der Ausdruck/der Satz zuvor [...] (ebd.)

So ist in (79) sowohl vor dem Korrelat als auch im korrelierten Nebensatz von was daraus wird die Rede. Korrelate in Mittelposition sind aber gleichzeitig echte Korrelate, durch die syntaktisch gebundene Kataphorik realisiert wird. Hegedűs (ebd.) weist auch darauf hin, dass die Distanz zwischen anaphorisch Bezug nehmendem Korrelat und vorangehender Bezugsgröße unterschiedlich ausfallen kann. So kann hier ein Beleg angeführt werden, in dem zwischen Korrelat und Bezugsobjekt mehrere (darunter zusammengesetzte) Sätze stehen:

(80) Von da ab wird mir berichtet vom Schwabenland, ich stellte es mir natürlich so schön vor, dass mich niemand davon abhalten konnte nicht mitzugehen. Auch beim Zehrpfennigsammeln habe ich mitgeholfen. Es war erlaubt, dass die Kinder, die ins Schwabenland müssen, in den Nachbarsgemeinden Geld sammeln dürfen für die Reise, damit die Kinder nicht den ganzen langen Weg zu Fuss machen müssen. Auch da hab ich immer am meisten bekommen, weil ich das jüngste von den Schwabenkindern war. Heimlich hat es mich dann doch oft gereut, dass ich gesagt habe, ich wollte auch mit, hab manche Träne nachts im Bett vergossen und hatte zum voraus schon Heimweh. (SCHG)

Der Inhalt des Kursivierten am Anfang von (80) steht dem des Korrelierten nahe. So bezieht sich das es anaphorisch auf das Mitgehen und gleichzeitig ist es Korrelat zu dem dass-Satz mit dem gleichen Inhalt. Gleichzeitiges Vorliegen von Ana- und Kataphorik ist nicht auf Korrelate zu beschränken, man vergleiche etwa folgendes Beispiel:

(81) daß es der ewige Schlaf ist, den sie schläft, daß sie - kaum erwachen werde. Und so war es gekommen. Sie - ist um 6 Uhr eingeschlafen, hat bis Mitternacht noch geathmet, $u$. ist dann verschieden. $(24)(\mathrm{K})$ 
Sowohl das dem es Vorausgehende wie auch das Nachfolgende handeln vom ewigen Schlaf. Es handelt sich bei dieser Eigenschaft von es also nicht um ein spezifisches Merkmal, das nur bei Korrelaten anzutreffen ist. Das Merkmal Verweis in zwei Richtungen ist grundsätzlich verfügbar und kann auch bei syntaktisch gebundener Phorik Anwendung finden. In Anlehnung an Schmidt (1987: 302) möchte ich Korrelate in Mittelposition und auch andere es-Vorkommen, deren Bezugnahme in zwei Richtungen erfolgt, biphorisch nennen:

Unserer Auffassung nach erfüllt das ES in den Fällen, wo es fakultativ ist, die Funktion eines Scharniers. Es verweist in zwei Richtungen. Einerseits verweist es satzintern auf die nachfolgende Information. Aber gleichzeitig signalisiert das ES, daß diese Information aus dem Prätext stammt. Das ES dient dazu, daß die comment-haftigkeit, die relative Neuheit der in der daß-Phrase (bzw. der Infinitiv-Phrase) auftretenden Information zurückzunehmen. [Verschränkung von dass-Satz und IK im Original, D.C.] Diese Eigenschaft, nämlich die Eigenschaft, in beide Textrichtungen zu weisen, nennen wir biphorisch. ${ }^{68}$

Mit dieser Auffassung ist die Bezeichnung des fakultativen Korrelat-es als Vorerwähntheitssignal (Zitterbart 2002a: 59; 2002b: 189, 192) kompatibel. Bei der kurzen Überblicksdarstellung zur Obligatorik/Fakultativität des Korrelat-es habe ich bereits die These angeführt, nach der die Setzung eines grundsätzlich fakultativen Korrelates mit der Thematizität des Korrelierten zusammenhänge (Kemme 1979: 20). Das Konzept von Biphorik ist also eine konsequente Weiterführung des Thematizitätsgedankens, indem nicht nur gesagt wird, dass das Korrelierte thematisch ist, sondern von tatsächlich vorhandener Anaphorik gesprochen wird, was Thematizität natürlich nach sich zieht, da Anaphorik per definitionem die Orientierung auf bereits Eingeführtes bedeutet.

\subsection{Empirie: Fallanalysen}

In diesem Kapitel sollen Belege präsentiert werden, durch deren Analyse eine Ergänzung und Präzisierung der Festlegungen in den vorangehenden Kapiteln vorgenommen werden kann. Dabei stehen folgende Bereiche im Mittelpunkt:

${ }^{68}$ S. hierzu auch Günthner (2009: $31 \mathrm{ff}$.). 
- Verweise auf Gegenstände

- Verweise auf durch NPs eingeführte Propositionen

- $\quad$ spezifische Korrelate: Spaltsätze

Zunächst werden Belege für Gegenstandsbezüge angeführt, dann solche für propositionale Verweise. Im Anschluss daran soll das in 2.3 geschilderte Korrelat-Bild durch funktionale „Verwandte“ erweitert werden. Bei Gegenstands- und Propositionsbezügen geht es in erster Linie um die Frage der morphosyntaktischen Realisierung, im Falle der Korrelate wird der Akzent auf korrelatähnliche Strukturen bzw. ihr Verhältnis zum klassischen Korrelat (vgl. 2.3.) gelegt.

Beim Verweis auf Gegenstände stehen NPs im Vordergrund. Aus grammatischer Sicht ist dabei von Belang, ob die Bezugnahme mit Korrespondenz zwischen es und Bezugsglied in Numerus und Genus einhergeht oder aber in Genus und/oder Numerus gegen diese Übereinstimmung „,verstoßen wird“ bzw. die Bedingungen einer Korrespondenz dieser Art gar nicht erfüllt sind, weil bspw. auf Einheiten, etwa ADJP, verwiesen wird, die hinsichtlich der Kategorisierungen Genus und Numerus nicht spezifiziert sind. Ein Spezialfall nichtkorrespondierenden Bezuges liegt bei der Verwendung des es in Kopulakonstruktionen vor. Zunächst werden NP-Bezüge

\section{NP-Bezug mit Genus-/Numeruskorrespondenz}

Der von Askedal (1990: 214) als „unter kategorialem Aspekt der systematische Normaltyp“ bezeichnete anaphorische NP-Bezug, bei dem der Kern der NP ein Substantiv im Neutrum Singular ist, findet sich in allen untersuchten Korpustexten und in jedem Zeitabschnitt:

(82) kein Brodt wahr auff dem Landt zu bekomen, dan es wahr auffgezertt von den Soldaten $(54 \mathrm{r})(\mathrm{G})$

(83) Mein Pferd war das Schießen nicht gewohnet und ging mit mir durch, wie ein Holländer. Da war kein Haltens. Weil im Trinken den Zügel fallen lassen, so hatte es seinen freien Lauf. (16) (MD) 
(84) Erst machte das Ding Bedenklichkeiten; nachwerts bot es sich selber an. (9) (B)

(85) da sagt mein mann | in seiner heimat stände ein schönes haus zu verkaufen. es stunde an der land-straße | es gehörte dem fürst von witgenstein (7) (DM)

(86) Ich suchte das Kind zu schützen, indem ich es in mein Zimmer mit dem Strickzeug setzte u. ihr nachher auch dort Stunde gab. (104) (SCH)

(87) Mir ist es halt nicht gegönnt, Dir dies Ringl so meuchlings an die Hand zu stecken, wie Du's mir gethan, denk' aber indem Du es an Deinem Finger siehst, (68) (LB)

(88) Ich halte es also für unwahrscheinlich, daß dieses ständig geübte Vokabular nicht auch in der Familie auftauchte. Das geht wahrscheinlich gar nicht anders. ... Und es tauchte auch auf. (17) (SB)

Diese Liste kann um einen Beleg erweitert werden, in dem durch das neutrale es auf Metasprachliches verwiesen wird:

(89) Aber ganz bewußt ... viele Dinge. ich weiß nicht, ob 'Traktor' deutsch ist ... 'Traktor' ist russisch. Ich weiß noch, daß damals, manchmal ein bißchen lustig, aber es ging dann plötzlich in den Sprachgebrauch über (18) (SB)

Das Wort Traktor ist zwar kein Maskulinum, es wird jedoch metasprachlich (im Sinne von „das Wort Traktor") verwendet und wird hier dementsprechend zum kongruenten Typ gerechnet. Die Gruppe kongruierender pro-phrasaler es-Vorkommen umfasst weiterhin insofern mehr als die klassischen Beispiele oben und in der Fachliteratur, als a) bezüglich der Bezugs-NP mit kategorialer Varianz zu rechnen ist und b) es in meinem Korpus als nähesprachlich einstufbare spezifische es-Vorkommen gibt, die auch ein es enthalten.

ad a)

Betrachtet man Beschreibungen über Merkmale des kongruenten Typs, so findet man i.d.R. Beispiele wie (82) bis (88), in denen der Kern der Bezugs-NP ein Substantiv ist. Dabei liegt hier offensichtlich Varianz vor, indem nicht nur NPs im engeren Sinne - d.h. NPs mit 
substantivischem Kern -, sondern auch NPs mit pronominalem Kern möglich sind. In meinem Korpus sind folgende Klassen von Pronomina belegt:

Indefinita im engeren Sinne $e^{69}$

(90) Das Gepolter im Haus währete ziemlich lange, bis endlich etwas zur Treppen herunterkam, über das Haus, im Hof zu plumpen anfing. Jch wußte, daß sonst kein Mensch im Hause war. Deswegen die Furcht und Angst sich mehrete. Aus dem Hof kam es rein in die Küche. (15) (MD)

(91) «Was hast am Finger? Zeig, es wird bald reif, wärme fleissig auf, füttere die Gäns im Stall und bleib daheim!> (14) (SCHG)

(92) kamen auch bißweilen aus andern dörffer meine bekanden, und fragten beÿ mir umb diß und das, so sagt ichs ihnen nach aller einfald aus unsern Gothaischen Catechißmo (19) (NE)

(92) ist insofern problematisch, als formal gesehen eine Verknüpfung der beiden Demonstrativa di $\beta$ und das vorliegt. Unter semantischem Aspekt und entsprechend der funktionalen Leistung von „diß und das“ wird dieser Beleg jedoch den Indefinita im engeren Sinne zugeschlagen, da die Bedeutung von „diß und das“ als Ganzem m.E. eher der von etwas/einiges ähnelt und daher eine indefinite Bedeutung angenommen werden kann.

\section{Quantifikativa $^{70}$}

(93) Doch alles pfützennaß, daß's im Augenblick, wie ein Harnisch, gefroren. (7) (MD)

(94) Jedes sagte, was es gethan und gerathen, um mich wieder bey ihnen zu haben. (7) (B)

\footnotetext{
${ }^{69}$ Indefinita im engeren Sinne (Typ: etwas, jemand bzw. Interrogativa) erfüllen ,,prototypischerweise die Funktion der indefiniten Referenz [...], ohne dass Beschränkungen im Hinblick auf die Größe der Extension des Nominals ausgedrückt würden.“ (Zifonun 2007: 9)

${ }^{70}$ Quantifikativa gehören nach Zifonun (ebd.) zu den Indefinita im weiteren Sinne und sind u.a. dadurch zu charakterisieren, dass sie im Gegensatz zu Indefinita im engeren Sinne die Größe der Extension des Nominals einschränken.
} 
(94) zeigt eine Verwendung von jed-aus der zweiten Hälfte 18. Jahrhundert, die heute im Standarddeutschen wohl nicht mehr möglich ist. Die neutrale Form ,,jedes“ bezieht sich eindeutig auf Personen und wird dann durch das grammatisch entsprechende es wiederaufgenommen.

\section{Demonstrativa}

(95) dieses alles württe mir eine neue mühe machen, wen ichs ein mal schreiben wolde. weil ichs aber dem Herrn professor Francke vor etligen Jahren schon neben andern dingen zu geschickt, sie werdens auch wol finden. (30) (NE)

(96) Will also nur sagen, können wir nicht das, was Du für Patscherei ansahest, auch so ansehen, wie ichs eben ansah, oder müßen wirs unwedersprechl für Patscherei nehmen? (16) (L)

In (95) finden wir ein durch alles erweitertes Demonstrativum, in (96) wird das durch einen Nebensatz näher bestimmt. Über Indefinita und Demonstrativa hinausgehend ist selbst die Proform es als Bezugsgröße möglich. Grundlage der Bezugnahme in (97) ist ein Subjekt-es in einem Spaltsatz:

(97) Ists nicht Wirklichkeit, sonder nur Schein, was mich zu reden trieb, so hats natürlich gar kein Gewicht (18f.) (L)

Auf dieses und andere ähnliche, funktional verwandte es-Vorkommen (Korrelate) wird weiter unten näher eingegangen.

Die Varianz hinsichtlich der Bezugsphrase kann auch darin bestehen, dass auf NPs in PPs Bezug genommen wird:

(98) Im Jahr 1646 da zoge die schwedische Armee hir im Land herumb und verderbten es jämerlich (17) (BL)

(99) Von da ab wird mir berichtet vom Schwabenland, ich stellte es mir natürlich so schön vor, dass mich niemand davon abhalten konnte nicht mitzugehen. (3) (SCHG) 
Die Grenzen der „klassischen“ Bezugnahme durch es können auch dadurch ausgedehnt werden, dass nicht direkt NPs als Bezugsglied fungieren, sondern bspw. Kompositionsglieder wie in (100):

(100) So halfterte ich mich endlich von dem Wasserholen los, auf die Art: Jch thät, als stolperte ich mit den beiden vollen Wasserhosen und goß's also über den ganzen Saal und Haus, daß alles schwamm. (6) (MD)

Dass der obige Beleg hier behandelt wird, wird darauf zurückgeführt, dass die wichtigen grammatischen Bedingungen, nämlich Korrespondenz in Numerus und Genus, weiterhin erfüllt sind, weil eindeutig „Wasser“ als Bezugsgröße fungiert, s. auch Behaghel (1923: 277).

ad b)

Grundsätzlich sind hier Links- und Rechtsversetzungen (Ágel/Hennig 2006: 393, 395 und Hennig 2006: 206f.) zu erwähnen. Bezogen auf es besteht der wesentliche Unterschied zwischen diesen beiden spezifischen Konstruktionen darin, dass die Verweisrichtung bei Rechtsversetzungen wie in (101) kataphorisch, bei Linksversetzungen wie (102) jedoch anaphorisch ist:

(101) Sobald ich das Tier nicht richtig gerupft hab, so hatte es gejuckt und geschrien. An manchem Ort hats geblutet, das arme Tier. (13) (SCHG)

(102) Dieses Ewig, ich kann es nicht ganz fassen, der menschliche Verstand ist zu schwach um etwas Unendliches zu fassen. (2) (K)

Gemeinsam ist beiden Konstruktionen, dass die Bezugnahme durch es auf ein Element erfolgt, das weder in einem anderen Satz noch in einem Teilsatz innerhalb eines zusammengesetzten Satzes, sondern sozusagen am Rande des es-haltigen Satzes steht. Der Rechtsversetzungs-Typ kommt auch bei Askedal (1990: 214, dort Typ 2) vor. Dabei merkt er an, dass er in seinem Korpus keinen einschlägigen Beleg gefunden hat und dass solche es-Vorkommen vornehmlich in gesprochener Sprache anzutreffen seien (ebd.). Meine Daten bestätigen die Annahmen Askedals. 
Rechtsversetzungen können definiert werden als „satzinterne Strutkur[en] am rechten Satzrand, die im Gegensatz zum Nachtrag Bestandteil der Projektionsstruktur des vorangegangenen Satzes [sind]“. (Ágel/Hennig 2006: 395) Darüber hinaus besteht an morphologischer Übereinstimmung erkennbare Referenzidentität mit einem Bestandteil des Bezugssatzes (Hennig 2006: 208), in (101) zwischen (dem klitisierten) es und „das arme Tier“. Dass Rechtsversetzungen als satzinterne Strukturen angesehen werden, mit denen keine neuen Projektionen verbunden sind, bedeutet für uns im Falle von (101), dass das Subjekt des Verbs bluten doppelt realisiert wird, was aber nicht heißt, dass das Verb etwa zwei verschiedene Valenzpotenzen realisieren würde und dadurch etwa zwei eigenständige Sätze entstünden. ${ }^{71}$ Die spezifische Leistung des es in solchen Strukturen besteht darin, dass es durch die Rechtsversetzung Einheiten vorwegnehmen kann, mit denen es zwar referenzidentisch, nicht aber morphologisch kongruent ist, was wiederum eines seiner bekannten Merkmale ist. Auf diese Fälle soll im nächsten Kapitel eingegangen werden.

Für Linksversetzungen gelten die gleichen Indizien wie für Rechtsversetzungen, bloß stehen sie eben am linken Satzrand. Auch sie sind satzintern und referenzidentisch mit einem Element im Bezugssatz. Von Funktionsidentität zwischen ihnen und ihrem „rechten“ Paar kann allerdings nicht die Rede sein, da die beiden Strukturen unterschiedlich motiviert sind. In (101) wird zunächst ein Element des Sachverhaltes durch es neutral eingeführt und dann am rechten Satzrand präzise benannt, während am linken Satzrand in (102) erst ein konkretes Referenzobjekt erscheint, über das in der Fortsetzung eine Aussage gemacht wird, in der das Bezugsobjekt „nur noch“ anaphorisch wiederaufgenommen wird.

Zusammenfassend lässt sich sagen: Für es-haltige Links- und Rechtsversetzungen ist nicht nur typisch, dass sie vorrangig in gesprochener Sprache anzutreffen sind, sondern sie stellen außerdem eine Gebrauchsweise von Ana- und Kataphern dar, die sprachübergreifend nur beschränkt möglich sein soll:

Die Anapher greift ein Antezedens auf, das in einem eigenen - meist vorausgehenden Textsatz erscheint oder aber sie erscheint in einem eigenen Teilsatz: Der Mann kommt. Er trägt einen schwarzen Hut. versus Der Mann trägt einen schwarzen Hut, wenn er kommt. Die Kookkurenz von Antezendens und referenzidentischem Personalpronomen innerhalb eines einzigen Teilsatzes hingegen ist nur beschränkt möglich. Hier ist das

\footnotetext{
${ }^{71}$ Eine ausführliche Darstellung des Begriffes Projektion soll hier nicht geleistet werden, vgl. aber zum (syntaktischen) Projektionsbegriff Hennig (2006: 192).
} 
Reflexivum erste Option: Der Mann ${ }_{\mathrm{i}}$ kauft sich $_{\mathrm{i}}{ }^{*}{ }^{i h m_{\mathrm{i}}}$ einen schwarzen Hut. (Zifonun 2001: 55)

Es könnte diskutiert werden, was genau unter Teilsatz zu verstehen ist und ob Satzrände als Teilsätze gelten. Im Sinne der obigen Überlegungen gelten sie als satzintern und stellen dementsprechend die im vorangehenden Zitat angesprochene beschränkte Möglichkeit dar.

Abschließend soll noch ein interessanter Beleg aus der ersten Hälfte des 18. Jahrhunderts angeführt werden, in dem es in Objektfunktion am Satzanfang erscheint:

(103) da sagt mein mann | in seiner heimat stände ein schönes haus zu verkaufen. es stunde an der land-straße | es gehörte dem fürst von witgenstein - es häten drei alte fräuleins bewohnt | wovon die eine noch lebte (7) (DM)

Auffallend ist die Anfangsstellung insofern, als sie im heutigen Deutsch generell ausgeschlossen ist (vgl. etwa Askedal 1990: 213). Dies kann darauf zurückgeführt werden, „dass die Vorfeldposition für pronominale Nicht-Subjekte eine exponierte Stelle darstellt, der das grundsätzlich schwache, morphologisch wie phonetisch nur über geringe Substanz verfügende es hier nicht genügen kann.“" (Zifonun 2001: 117) ${ }^{72}$ Theoretisch möglich wäre es also - ausgehend von (103) - anzunehmen, dass der grundsätzlich schwache Charakter des es historisch gesehen nicht immer rigorose Stellungsregularitäten zur Folge hatte oder umgekehrt dass seine heutige „Schwäche“ sich erst im Laufe der Zeit entwickelt hat und es die Vorfeldposition auch in nicht-subjektischer Funktion besetzen konnte, genau so wie sein feminines und maskulines Pendant (sie und er) dies heute noch tun. Da aber in meinem Korpus nur dieser einzige Beleg gegen die Gegenwartsregel spricht, können diese Annahmen bezogen auf die vorhandenen Belege nicht weiter verfolgt und verifiziert werden.

\section{NP-Bezug ohne Genus-/Numeruskorrespondenz und Bezüge auf ADJP/VP}

Ein nicht korrespondierender NP-Bezug liegt vor, wenn auf NPs verwiesen wird, deren substantivisches Glied mit es morphologisch, d.h. in Numerus oder Genus, nicht übereinstimmt. Möglich sind hier a) Bezugnahmen auf Maskulina und Feminina sowie auf pluralische Elemente einschließlich der Möglichkeit mehrerer, koordinierter singularischer

\footnotetext{
${ }^{72} \mathrm{Vgl}$. hierzu auch IDS-Grammatik (1997: 1585).
} 
oder pluralischer Einheiten, aber auch nicht-NP-Verweise, d.h. b) prädikativische esVorkommen, bei denen bspw. Bezugsadjektive hinsichtlich Genus und Numerus nicht spezifiziert werden können und daher die Rede von (fehlender) Korrespondenz sich erübrigt, und schließlich c) spezifische es +Kopula $+N P$ - und ähnliche Verbindungen, die hauptsächlich auf der Basis ihrer besonderen textuell-pragmatischen Leistung erklärt werden können.

ad a)

Die Proform es ermöglicht anaphorische und kataphorische Verweise, bei denen zwischen es und dem Bezugsglied morphologisch gesehen keine Korrespondenz in Numerus und Genus vorliegt. Dies ist zweifellos eines seiner wichtigsten Spezifika im phorischen Bereich, das es gegenüber maskulinem er und femininem sie auszeichnet. In meinem Korpus sind Belege für alle oben erwähnten nicht korrespondierenden Fälle anzutreffen:

Bezug auf pluralische NPS

(104) Wir in Stausenbach musten in geben 120 Reichstaller. Ach wie mancher vergoß die heisen Trenen daruber, biß wir es zusamen brachten. (8) (BL)

(105) so waren wir einem manne | der schon Viel geschalte mit uns gemacht hate - nur noch 13 Reichstaler schuldich - er hate uns eingeklagt | was ich aber nicht wuste müller hatte die schrift verstekt - er ließ die 2te und 3te schrift komen. ohne das ich es wuste häte er es nun auch heimlich bezahlt | so häte sein verheimligen doch einen grund gehabt (6f.) (DM)

(106) Ich hatte das Mal in Kirchain elf Stuck Rindvieh und 17 Schwein und 4 Zigen. Wir musten gar vleisig wachen die Nacht, das es uns nicht gestollen ward von den Soltaden (14) (BL)

(107) Bey ihme hatte ich Essen und Trincken, so ich es nuhr recht genießen kundte. (60r) (G)

In (104) nimmt es auf eine NP Bezug, die aus einem Substantiv im Plural und dessen attributivem Zahladjektiv besteht, in (105) sind es zwei koordinierte singularische NPs. 
(106) ist ein Beispiel für Aufzählungen, deren Bestandteile sehr unterschiedlich gestaltet sein können. (107) stellt ein Beispiel für so genannte artikellose „formelhafte Wortpaare“ (Duden 2005: 1016) dar, das hier wegen seiner formalen Pluralität angeführt wird. Typisch ist aber für solche Verbindungen, dass sie inhaltlich oft als Einheiten interpretiert werden und eine Verbform im Singular fordern. Es ist also möglich, auf Grund semantischer Überlegungen eine singularische, neutrale Einheit anzunehmen, wodurch dieser Beleg als ein Repräsentant des korrespondierenden Typs aufgefasst werden könnte. Ähnlich lässt sich bezüglich (108) argumentieren, in dem sich allerdings ein artikelhaltiges Wortpaar befindet:

(108) Das Bier und Wasser in dem Städtchen wurde vom Viehe und so viel Menschen ganz ausgesoffen, daß nichts mehr zu bekommen war, und wir von weitem es holen mußten. (16) (MD)

Bezug auf Feminina

(109) Die Zeit ist wohl wieder recht gedrängt, aber wir werden's uns schon eintheilen, nicht wahr? (1787) (LB)

(110) Zogen auch vor den Kirchain und wolten den einnehmen. Schossen auch uff 3 hundert Schüß mit Stucker wider die Mauer und in die Stadt. Schoßen auch die Mauer bey dem stumpen Thurn über ein Hauffen, aber sie liffens nicht an mit Sturm (13) (BL)

In (110) sind sowohl Stadt als auch Mauer als Bezugsglied vorstellbar, aber da beide Feminina sind, spielt hier diese Interpretationsfreihet keine Rolle.

Bezug auf Maskulina

(111) Der ließ die Kandtengießergesellen bey $\mathrm{i}[\mathrm{h}] \mathrm{me}$ nach des Handtwercks Prauch einziehen, gabe mihr Essen und Trincken, behielte mich uber Nacht, stellet mihr einen Schuncken auff. Dißer wahr voler Maden, dick wie ein Spindelspitz. Es geschmecket mihr aber woll (85v) (G) 
(112) Also das ist Alles vom Balle. Die 1. Mutter u 1. Gabi waren mit mir, eine seltene Ehre für den Ball. Jedenfalls gefiel es mir besser als voriges Jahr (2) (K)

Bei (112) kommt auch eine Interpretation in Frage, nach der es als Teil des (okkasionell) impersonalen Ausdrucks es gefällt jm. X-wo, aber durch das Fehlen des X-wo-Teiles (s. dazu die okkasionellen Ereignisverben mit es und PP in 3.4) lässt sich die andere Deutung, nämlich der Bezug auf eine NP mit maskulinem Kopf, meiner Meinung nach gut rechtfertigen.

Im Korpus sind auch Rechtsversetzungen vertreten, bei denen der Bezugsausdruck in der Rechtsversetzung mit es zwar koreferent ist, mit diesem aber morphologisch nicht übereinstimmt:

(113) Ich kan es gar nicht umbgehen und vergeßen, wie zuvor gedacht, der verwüsten und zerrisene Heuser und Bäuen in Stausenbach. (16) (BL)

zwei Gänserich haben mich geweckt. Ich habe mich herzlich gefreut und habs gestreichelt die zwei Tiere. (16) (SCHG)

In diesen Sätzen erfüllt es vor der jeweiligen Rechtsversetzung die Funktion, den später zu benennenden Referenten zunächst neutral zu bezeichnen und diesen auf diese Art einzuführen. Das Fehlen von Genus- und Numeruskorrespondenz ist gegenüber anderen Kataphern (und auch Anaphern) ein besonderes Merkmal von es, schließlich werden allgemein für Rechtskontexte bspw. in der IDS-Grammatik (1997: 548) solche Korrespondenzen postuliert.

ad b)

In 2.2.2 wurde der besondere Status von Verweisen auf Eigenschaften erläuert. Dieser Sonderstatus - nämlich dass solche Bezüge zwischen propositionalen Verweisen und solchen auf Gegenstände zu verorten sind - ergibt sich einerseits aus der semantischen Bestimmung von Eigenschaften (vgl. Asher 1993 und Zifonun 2001) und den syntaktischen Merkmalen prädikativer Strukturen mit einem Kopulaverb. Außer den Belegen in 2.2. sein an dieser Stelle noch folgendes Beispiel angeführt: 
(115) Doch bekam er jetzt wieder in Aussig einen Posten, hoffentlich einen sicherern, als der letzte es war. (10) (K)

(115) ist insofern ein Sonderfall, als das Adjektiv - ähnlich wie die NPs in (98) und (99) Teil der Bezugsphrase, hier einer NP, ist. Wichtig ist es an dieser Stelle darauf erneut hinzuweisen, dass der Bezug auf Prädikative durch es von dem auf NPs ohne Korrespondenz in Genus und/oder Numerus (s. a)) sauber getrennt werden muss, da eine Korrespondenz bezüglich der erwähnten nominalen Kategorisierungen im Falle eines Verweises auf ADJPs keine Option darstellt, sondern grundsätzlich nicht gegeben ist. Des Weiteren sind auch Kopulasätze mit es und einer NP anders als die NP-Bezüge unter Punkt a) zu handhaben, vgl. c) weiter unten.

$\operatorname{adc}$

Nicht alle Kopulasätze, die ein es enthalten, sind generell als spezifisch einzustufen, man vergleiche etwa folgenden Beleg, in dem durch das kongruente es auf eine NP verwiesen wird:

(116) Der einte war, daß ich noch viel zu wenig Holz hatte, ungeachtet Mstr. Brunner mir gesagt, es sey genug (12) (B)

Gemeint sind vielmehr Sätze mit einem Kopulaverb, die über eine besondere Funktion verfügen. Diese Funktion entsteht immer im gegebenen syntaktischen Rahmen. Es sind syntaktische Ausdrucksmodelle im Sinne von Feilke (1996: 241), die der Charakterisierung und Identifizierung dienen (Askedal 1990: 214 und Zifonun 2001: 77). Dabei wird der Gebrauch des es auch in nicht kongruenten Fällen bevorzugt, was auf eine feste Konstruktion hinweist. Dies soll durch folgende Beispiele veranschaulicht werden: der bekam abschlächlige antwort und wens auch ein Kavalir wahr er hate nun Rang und geld (2) (DM) 
(118) In dem ersae ich weitt vohr mihr etwaß fohr mihr ston, ungevohr ein halbe Meil Wœgx weitt. Als ich $i[h] n$ erkandte, das es ein Man wahr, erschracke ich zim Teil $(91 \mathrm{v})(\mathrm{G})^{73}$

Eine Änderung der Konstruktion würde dazu führen, dass wir bspw. im Falle von (117') die kongruente Pro-Form wählen müssten:

(117') der bekam abschlächlige antwort und wenn *es/er sich an jemanden anders gewandt hätte, hätte er auch...

Auch Back (1995: 161f., Fußnote 39) kommentiert kurz diese Eigentümlichkeit. ${ }^{74}$ Es gibt auch semantisch ähnliche Fälle, in denen eine Charakterisierung nicht durch sein, sondern bspw. durch halten für erfolgt. Inhaltlich-funktional sind sie aber analog:

Joseph sprach zu dem obersten schencken, und becker, auslegen der treume gehöret Gott zu, so hab ichs auch meinem Gott überlaßen der am besten wuste, was dieser traum beteuten wird, nur nach meiner Einfald habe ichs recht vor einen Göttlichen traum /gehalden/ (8f.)

Weiterhin kann als Hinweis auf eine feste Konstruktion zu deuten sein, dass auch in für deiktische Prozeduren typischen Kontexten ein es statt das erscheinen kann:

Bea, Telefon für dich. Wer ist es? (Bethke 1990: 182)

Schließlich soll an dieser Stelle auf die Überlegungen zur Thetizität in Kapitel 2.2.3 verwiesen werden. Dort wurde die Möglichkeit erwogen, im Falle spezifischer Kopulasätze mit es in Analogie $\mathrm{zu}$ anderen thetischen es-Vorkommen Thetizitätsmarkierung anzunehmen.

\footnotetext{
${ }^{73}$ Der Beleg erinnert im Übrigen an nähesprachliche Apokoinu-Strukturen, indem zwei mögliche kategoriale Realisierungsformen des vom Verb erkennen geforderten direkten Objekts gleichzeitig auftreten: Das akkusativische Pronomen (ihn) und der Objektsatz (das es ein Man wahr) werden beide realisiert.

${ }^{74} \mathrm{Er}$ argumentiert, dass die Wahl zwischen kongruenter Pro-Form und es vom Bezugspunkt abhängt: „Im Unterschied zu den bestimmten persönlichen Pronomen weist ES auf den komplexen Inhalt des Gesamtsatzes oder eines großen Teils desselben hin“. Dabei verweist er auf Fußnote 11 (ebd., 151), in der als analoges Beispiel Folgendes zu lesen ist: „Cäsar versprach den Römern einen neuen Krieg im Osten. DAS/ES war kurz vor seiner Ermordung." Sein Argument und die Parallele sind m.E. allerdings falsch, denn in seinem Beispiel in Fußnote 39 bezieht sich es auf einen durch eine komplexe NP eingeführten Gegenstandsentwurf, während im Cäsar-Beispiel ein Verweis auf eine Proposition (eingeführt durch einen Elementarsatz) vorliegt.
} 


\section{Phrasale Verweise auf Propositionen}

Im theoretisch ausgerichteten Kapitel 2.2.3 wurden Belege angeführt, die propositionale Verweise darstellen. Dabei wurde der Akzent auf Elementarsätze gelegt. An dieser Stelle sollen Belege gezeigt werden, die von den typischen Fällen abweichen. In erster Linie ist dabei an sententiale Nominale (,,sentential nominals“, vgl. Asher 1993). In meinem Korpus finden sich bspw. Nominalisierungen von Verben. Sie sind deshalb interessant, weil sie als konvertierte Verben abstrakte Objekte darstellen:

(120) da gieng's zuerst an ein lermendes, von Zeit zu Zeit mit Thränen vermengtes Freudengeschrey von Kleinen und Grossen, dann an ein Bewillkommen, Betasten und Begucken, Fragen und Antworten, daß es eine Tausendslust war. (7) (B)

(121) Ich habe nicht Zeit zum Schreiben aber ich fühlte dieser Tage so das Bedürfniß es zu thun u. kann nicht dazu kommen. (1) (K)

Dabei sind die kursiv gesetzen Bezugsausdrücke unter morphosyntaktischem Aspekt jedoch keine Elementarsätze, sondern NPs. So ist in (121) Schreiben eine NP (Syntax: phrasal), die wohl das Gleiche leistet wie die entsprechende IK zu schreiben (Semantik: propositional). (120) ist ein Beleg für summierende Verweise, bei der die summierten Propositionen durch NPs realisiert werden. Ebenfalls als phrasaler Bezug auf eine Proposition ist der Beleg (122) einzustufen, in dem auf einen Teil des Prädikates (das Vollverb) Bezug genommen wird:

wie mancher Punkt müßte von beiden Theilen besprochen werden u. wird's nur von dem einen. (108) (SCH)

Dabei ist die Bezugsphrase allerdings keine NP, sondern ein Teil einer VP. In 2.2.2 wurde dafür argumentiert, dass Bezüge dieser Art als propositional zu deuten sind.

\section{Spezifische Korrelate: Spaltsätze}

Als dritter Punkt in diesem Kapitel sollen Belege für spezifische Korrelate angeführt werden. In 2.3.3 wurden insgesamt sieben Stufen (Stufe 0 bis 6) der Integration angenommen. Dabei stand der Anfangspol, Stufe 0, für Anaphorik, bei der Elementarsätze 
syntaktisch in keinerlei Weise integriert werden. Am anderen Ende der Skala standen wennSätze in Komplementfunktion (Stufe 6). Dieses Stufensystem soll hier in verereinfachter und kurz kommentierter Form wiedergegeben werden:

- Nicht-Integration: Stufe 0

- Schwache Integration: Stufe 1 bis 3

- Starke Integration: $\quad$ Stufe 4 bis 5 (und 6 als Sonderfall, vgl. 2.3.3)

Dabei gilt, dass semantische Abhängigkeit des Korrelierten erst ab Stufe 1 angenommen wird, wobei die Integration durch die Syntaktisierung der semantischen Abhängigkeit erhöht wird (Zunahme an Statushomologie). Es handelt sich dabei darum, dass zwischen Stufe 1 und 5 unterschiedliche formale Kodierungen (so Inf. ohne $z u$, abhängige Hauptsätze, uneingeleitete Nebensätze, Nebensätze mit Verbletzt und Subjunktor sowie Inf. mit zu) semantischer Abhängigkeit zu sehen sind. In Anlehnung an die Analysen in Fischer (2009) sollen nun auch so genannte Spaltsätze in das obige Bild integriert werden. Belege für Spaltsätze finden sich auch in meinem Korpus:

(123) müller nahm mich in der angst - auf seinen rücken und lief mit der bürde die höhe hinab - in das Dorf schameder, es war ein leinwebers haus was vorne im dorfe stand (9) (DM)

(124) Endlich ver-Reißete der alte herr - und brachte einen neuen ofizianten mit namens Müller - dießer ach dießer mann war es | der so viel einfluß auf mein nach übriges leben haben sollte (1) (DM)

(125) Aber es waren auch 2 traurige Wochen, die auf den Ball folgten (1) (K)

(126) Ich schrieb ihr, wie Du weißt, ich meine, es sind wohl 4 Wochen, u. hab keine Antwort mehr erhalten (12) (L)

Spaltsätze vertreten nach Fischer (2009: 174) neben Betonung und Voranstellug eine wichtige Fokussierungsstrategie. Im Gegensatz $\mathrm{zu}$ intonatorischen Mitteln und der Bewegung von Konstituenten an den Satzanfang handelt es sich bei Spaltsätzen um eine syntaktische Konstellation, die Fokussierung ermöglicht. Dabei erfolgt Fokussierung mittels eines Relativsatzes, der an einen Kopulasatz mit es und einer NP angeschlossen wird. Die 
Position des es und die der NP ist dabei variabel, vgl. (123) und (124). Fischer (ebd., 179) nimmt dabei an, dass das es kataphorisch auf den Relativsatz verweist und daher über eine kataphorische Kraft verfügt. Folglich ist eine Analyse dieses $e s$-Vorkommens als Korrelat möglich. Wichtig sind allerdings auch die Differenzen zwischen klassischen es-Korrelaten und dem es in Spaltsätzen. Bspw. ist die Umstellung des Relativsatzes nicht möglich (ebd., 180), während korrelierte Komplementsätze an den Anfang bewegt werden können, wobei das Korrelat dann - mit Ausnahme der wenn-Sätze - wegfällt. Dies trifft für das es in Spaltsätzen nicht zu, das nicht wegfallen kann. Hinzu kommt, dass das am Ende von 2.3.3 behandelte Phänomen der Biphorizität bei Spaltsätzen bzw. der Interpretation von es nicht in Frage kommt. Dieses es ist immer nur kataphorisch und der Konstellation es + Kopula + $N P+$ Relativsatz kann eindeutig die Funktion Fokussierung zugeschrieben werden. Grammatisch besonders sind Spaltsätze auch deshalb, weil Korrespondenz in Numerus zwischen finitem Verb und der prädikativischen NP vorliegt, nicht aber zwischen es, das Fischer (2009: 170, 181, zur Diskussion s. auch Askedal 1990) als Subjekt interpretiert, und dem Finitum. Weiterhin besteht Korrespondenz bezüglich Genus und Numerus zwischen der Prädikativ-NP und dem Relativpronomen, vgl. (125). Diese grammatischen Besonderheiten sprechen m.E. dafür, Spaltsätze als einen besonders stark gebundenen Тyp von Kataphorik zu interpretieren. Diese Annahme wird durch (126) präzisiert: In diesem Beleg ist $\mathrm{zu}$ sehen, dass sogar diese funktional motivierte und syntaktisch besonders ausgeprägte Form gebundener Kataphorik abgeschwächt werden kann. Die Integration ist hier insofern niedriger, als statt eines NS, d.h. eines relativen Anschlusses, ein Elementarsatz mit und angeschlossen wird. 


\section{Das nicht-phorische es}

\section{1 es-Valenzsimulationen}

Im Untertitel ihres 1995 in der Deutschen Sprache erschienenen Aufsatzes bezeichnete Gisela Zifonun das nicht-phorische es als „Prüfstein grammatischer Theoriebildung“. Dies spiegelte die Tatsache wider, dass der eine „Zweig“ der einschlägigen Forschung sich intensiv mit dem syntaktischen Status von es und dessen theoretischer Beschreibung und Erklärung auseinandergesetzt hatte, was Konsequenzen für die jeweilige Grammatiktheorie nach sich gezogen hat. ${ }^{75}$ Ein anschauliches Beispiel hierfür war und ist die Behandlung von es in generativen Arbeiten, die dort einerseits zu der Annahme eines Wandels der strikten Subkategorisierung bzw. verschiedener Basisgenerierungsmöglichkeiten bei Verben führte (vgl. bspw. Pütz 1975, Lenerz 1985 und Cardinaletti 1990), andererseits die Etablierung leerer Kategorien („dummy“ oder „empty category“, vgl. Hoeing 1994) nach dem so genannten Leerstellenprinzip bewirkte, was wiederum bekanntlicherweise einen wesentlichen Beitrag zu der Diskussion über Position und Generierung des Subjekts in der X-bar-Theorie leistete (s. dazu Haider 1993: 132ff.).

Mit Zifonun (1995: 59) bin ich der Ansicht, dass die vorfindlichen generativen Beschreibungen des nicht-phorischen es nicht im Stande sind, dessen oberflächensyntaktisch ermittelbares grammatisches Verhalten adäquat zu erfassen. Daher ruft dieses Kapitel eine andere Grammatiktheorie, nämlich die Valenztheorie, zu Hilfe. Die folgenden Überlegungen gliedern sich in zwei Teile: Zunächst soll der Begriff der Valenzsimulation erklärt werden, wobei zwei Typen der Simulation angenommen und beschrieben werden. Darauf folgt ein Kapitel zum Korrelat-es, in dem ein möglicher Weg der Integration dieser es-Gebrauchsweise ins es-Gesamtsystem und eine Erweiterung bzw. Präzisierung der Valenzsimulationstypologie vorgeschlagen werden. Im Anschluss an die Ergebnisse der Analysen soll dann in 3.2 einer der Valenzsimulationstypen, nämlich der konstruktionelle, näher betrachtet werden.

Unter grammatiktheoretischem Gesichtspunkt ist die Berücksichtigung valenztheoretischer Überlegungen in der es-Forschung nicht neu. So finden sich vereinzelt valenzielle Informationen u.a. bei Erben (1979), Askedal (1990) und Helbig / Buscha (2001,

${ }_{75}$ Der andere „Zweig“ ist gekennzeichnet durch Versuche, die verschiedenen es-Gebrauchsweisen zu klassifizieren, vgl. u.a. Pütz (1975), Admoni (1976) und Askedal (1990). 
Kapitel 6) bzw. dependenziell-valenzielle in Eroms (2000: 188ff.). Für die Darstellung hier sind in erster Linie die Überlegungen Ágels (2000b: $228 \mathrm{ff}$.) relevant, da diese eine detailliertere Analyse nicht-phorischer es-Vorkommen bieten. Daher sollen sie im Folgenden dargestellt und weiterentwickelt werden.

Ágel bettet seine Auführungen zum nicht-phorischen es in den Kontext der strukturellen Valenzrealisierung ein (2000b: 228). Unter struktureller Valenzrealisierung versteht er „Formen und Typen der grammatischen Realisierung der Valenz in verschiedenen Einzelsprachen bzw. verschiedenen Varietäten derselben Einzelsprache [...]“ (ebd., 105) ${ }^{76}$ Bezogen auf nicht-phorische es-Vorkommen geht es darum, dass die in der herkömmlichen Terminologie formal (vgl. etwa Duden 2005: 830) oder expletiv (vgl. etwa Eisenberg 2006: 174) genannten es-Subjekte (bzw. gelegentlich -Objekte ${ }^{77}$ ) oberflächlich betrachtet so aussehen wie normale Erst- oder Zweitaktanten, in der Tat es aber nicht sind, da sie die normale Erst- bzw. Zweitaktantenrealisierung nur „simulieren“ (Ágel 2000b: 229), ohne dass dabei die betroffenen Verben über die entsprechende Valenzpotenz ${ }^{78}$ verfügen würden:

Valenzsimulationen sind ,als Nachahmungen von Valenzrealisierungsstrukturen ohne realisierte Valenz(potenz) zu definieren (ebd., Fußnote 27).

Dass der Erst- bzw. Zweitaktant nur nachgeahmt wird, erkennt man daran, dass die formalen es-Subjekte und -Objekte ,nicht Träger einer semantischen Rolle und in diesem Sinn semantisch leer“ sind (Eisenberg 2006: 174). In valenztheoretischer Begrifflichkeit heißt dies, dass auch die inhaltliche Spezifizität (INSP) der betroffenen Verben nachgeahmt wird, dass das es also in diesen Fällen „(auch) semantische Rollen simuliert, d.h. pseudoagentiv oder pseudo-patientiv ist. Unter simulierter Valenz ist hier also (auch) +PseudoINSP zu verstehen.“ (Ágel 2000b: 230) ${ }^{79}$ Das Paradebeispiel von Valenzsimulation stellen Witterungsverben (Typus: es regnet) dar. Im Sinne der obigen Ausführungen sind diese Valenzträger mit Simulations-es nullwertig, haben also kein Subjekt: „Des Subjekts Habitus wird gemimt, auch dort, wo es keines gibt“" (Faucher 1996: 46).

\footnotetext{
${ }^{76}$ Es is wichtig zu betonen, dass es hier nicht um die kontextuell-situative Realisierung der Valenz, also um das Verhältnis von Valenz und Text geht, sondern um Fragen der Sprachstruktur und deren Verhältnis zur Valenz (ebd.).

${ }^{77}$ S. hierzu u.a. die Beispiele in Duden (2005: 831).

${ }^{78}$ Unter Valenzpotenz ist die Fähigkeit von relationalen Sprachzeichen, die der Kategorie Verb angehören, zu verstehen, „die semantische und syntaktische Organisation des Satzes zu prädeterminieren.“ (Ágel 2000b: 105).

${ }^{79}$ Vgl. auch den Begriff „Pseudoaktant“ in Duden (2005: 830).
} 
Ágel (2000b: 230) thematisiert zwar vor allem Witterungsimpersonalia wie es regnet/schneit/blitzt, geht aber in Anlehnung an Szatmári (1998) auch auf andere es-Typen wie das Vorfeld-es oder das es in passivisch interpretierbaren sich-lassen-Gefügen ein. Unter Rückgriff auf diese ersten Ansätze zur Ausdehnung des Valenzsimulationsbegriffes auf weitere es-Vorkommen sollen auch folgende Belege angeführt und als Beispiele für Valenzsimulation interpretiert werden:

(127) eÿ höret doch liebe Mutter, es hat Ja ein Gottseliger Man diese bücher alle beide geschriben (3) (NE)

(128) Da ward es mir gar kalt im Leibe. (23) (MD)

(129) Der Großmutter geht es jetzt schon gut, doch hat sie diese letzten 2 Wochen viel gelitten (5) (K)

(130) [...] als es nun rund in der stuben glimmete, so fingen hernach am ende rechte flammen /an zu brennen/, das es recht helle brande [...] (8) (NE)

(131) es gibt schon leute in unserer gemeinde [...] (17f.) (NE)

(132) ferner hat mich die sonderbare führung des lieben Gottes so weith gebracht, in dem ich nun erkenne, das er es recht gut mit mir gemeinet wie er zu davit gesaget im 32 Psalm. (5) (NE)

In all diesen Fällen liegt ein semantisch leeres, Agentien bzw. - in (132) - Patientien nachahmendes es vor, wobei diese Belege in semantischer Hinsicht recht heterogen sind, man findet hier es-Vorkommen, die Naturerscheinungen, Zeitverhältnisse, Existenz usw. zum Ausdruck bringen (vgl. auch Helbig / Buscha 2001: 398ff.). Und obwohl sie in Anlehnung an Ágel (2000b: 229) alle Valenzsimulationen darstellen, sind sie nicht nur semantisch heterogen, sondern auch syntaktisch. Auch Ágel (ebd.) räumt ein, dass man hier „den teils differierenden Eigenschaften der nicht phorischen es-Typen Rechnung tragen [...]“ sollte. So stellt Szatmári (1998: 235ff.) bezogen auf die von ihr untersuchten sichlassen-Fügungen und andere es-Vorkommen (s. oben) fest, dass verschiedene Stufen der Subjekthaftigkeit von es anzunehmen sind in Abhängigkeit von dessen obligatorischer oder fakultativer Setzung bzw. Nicht-Realisierung im Vor- und Mittelfeld. Tatsächlich gilt, dass das Vorfeld-es bei Besetzung des Vorfeldes durch ein anderes Element obligatorisch 
wegfällt, während das fixe es in jeder Position vorhanden sein muss ${ }^{80}$ und das es in sichlassen-Gefügen unterscheidliche Verhaltensmuster zeigt (ebd.). An dieser Stelle muss darauf hingewiesen werden, dass unter „Subjekt-“ in „Subjekthaftigkeit“ im Sinne der obigen Überlegungen simulierte Subjekte zu verstehen sind. Um dem heterogenen Wesen nicht-phorischer es-Typen Rechnung $\mathrm{zu}$ tragen, zieht Ágel (2000b: 230) die Grammatikalisierungstheorie heran und postuliert eine Skala mit zwei „Extremen“:

Das eine Extrem stellen dabei primär nullwertige VT [Valenzträger, D.C.] dar, deren Valenzsimulation voll grammatikalisiert ist (Typ: es blitzt). [...] Das andere Extrem mit es nur im Vorfeld bilden Sätze, bei denen die Valenzsimulation keine INSP-Simulation beinhaltet. $^{81}$

In der Übergangszone zwischen diesen beiden Polen befinden sich nach Ágel (ebd.) Mittelkonstruktionen, darunter auch die von Szatmári (1998) untersuchten sich-lassenKonstruktionen mit optionalem es (Typus: Hier lässt es sich gut leben.) und ExperiencerImpersonalia mit (ebenfalls) optionalem es (Typus: mich friert (es) und (128)). Eine solche skalare Abbildung der Verhältnisse ist zwar einleuchtend, lässt sich aber historisch schwer untermauern, und zwar aus zwei Gründen:

1) Das funktionale Spektrum von es ist bereits zu althochdeutscher Zeit recht breit (s. Große 1990) und

2) es ist schwierig festzustellen, welcher Pol der von Ágel postulierten Skala den Anfang und welcher das Ende des das nicht-phorische es betreffenden Grammatikalisierungsprozesses darstellen soll.

Denn den Überlegungen Ágels entsprechend sollte das Vorfeld-es den Anfang und der voll grammatikalisierte Typ der Witterungsimpersonalia das Ende der Skala bilden, wobei u.a. die Experiencer-Impersonalia (s. (128)) mit es eine Zwischenstellung einnehmen würden. Dies ließe sich jedoch mit dem in der Fachliteratur dokumentierten Aufkommen der betroffenen es-Typen nicht in Einklang bringen. Denn einerseits steht dort, dass das fixe es bei Witterungsverben seit althochdeutscher Zeit belegt ist (vgl. etwa Behaghel 1923: 316f.),

${ }^{80}$ Kritisch kann hier angemerkt werden, dass es Verbspitzenstellungsphänomene gibt, wo dies nicht gilt, vgl. Auer (1993: 196f.).

${ }^{81}$ Für diesen letzteren Fall s. (127). 
während die Experiencer-Impersonalia mit es erst seit mittelhochdeutscher Zeit präsent sind (Ebert 1978: 55) und dann in neuhochdeutscher Zeit im Rahmen der Generalisierung der Subjektskodierung umstrukturiert werden, sodass ein semantisch leeres es eingeführt wird (Ágel 2000a: 1872 und Seefranz-Montag 1995: 1279). Andererseits wird in der einschlägigen Fachliteratur angenommen, dass das Vorfeld-es aus dem Korrelat-es abzuleiten ist (Brugmann 1917: 15ff., Große 1990: 31 und etwas differenzierter ${ }^{82}$ auch Vogel 2006: 190). Dabei ist das Vorfeld-es seit mittelhochdeutscher Zeit belegt (Ebert 1978: 57). Um die entsprechenden Grammatikalisierungswege und -stufen eindeutig bestimmen zu können, müsste das oben erörterte sprachhistorische Problem gelöst werden. Bevor dies geschieht, soll eine mögliche Präzisierung des Valenzsimulationsbegriffs bezüglich des nicht-phorischen es vorgenommen werden, um im Anschluss daran den chronologischen Einwand erneut aufzugreifen und eine Antwort auf die sprachhistorische Frage zu bieten.

Ausgehend von den oben kurz dargelegten differierenden syntaktischen Merkmalen nicht-phorischer es-Vorkommen werden in einem ersten Schritt zwei Valenzsimulationstypen angenommen:

Valenzsimulationstyp 1: positional: (127)

Valenzsimulationstyp 2: konstruktionell: (128) bis (132)

Gestützt wird diese Unterscheidung dadurch, dass gegenüber den Belegen (128) bis (132) im Falle des Vorfeld-es (s. (127)) „nur“ die Position des Subjektes simuliert wird (keine INSP-Simulierung), nicht aber seine semantische Rolle. Zifonun (2001: 81) spricht hier von „rhematisierenden Präsentationskonstruktionen“, die nicht der Wiederauf- oder Vorwegnahme von Bezugsobjekten dienen, sondern die Subjektposition am Satzanfang simulieren, um dadurch das Subjekt zu rhematisieren. Vogel (2006: 238) geht weiter und bringt das Vorfeld-es mit (sekundärer ${ }^{83}$ ) Thetizität in Zusammenhang. Thetizität ist ein so genanntes monolitisches Konzept, das durch eine reine Satzaussage charakterisiert ist (ebd., 69, 114). Typische Beispiele hierfür sind Witterungsimpersonalia und in einem zweiten Schritt auch Passiva. Das Pendant hierzu stellt Kategorizität dar, das ein dichotomisches Konzept darstellt, „das sich funktional gesehen auf Satzgegenstand und -aussage

\footnotetext{
${ }^{82}$ Vogel nimmt an, dass heutigen Spaltsätzen (Typ: Es ist Peter, der heute nicht kommt.) ähnliche Fügungen mit textreferentiellem es, Kopula und Prädikatsnomen den Ausgangspunkt bildeten, wobei im Falle von Vollverben statt Kopula eine syntaktische Reanalyse stattfand, sodass das ehemals echte Subjekt-es zu einem reinen Vorfeldmarker wurde.

${ }^{83}$ In diesem Fall ist „,sekundär“" sowohl strukturell als auch historisch zu verstehen, s. Vogel (ebd.).
} 
gleichermaßen stützt [...]“ (ebd., 114). Typische Beispiele hierfür sind Aktivsätze mit transitivem Verb (und Agens und Patiens). Während Thetizität konzeptuell grundsätzlich mit „Event-Zentriertheit“ (reine Prädikation mithilfe eines Geschehensverbs) verbunden ist, sind kategorische Sätze grundsätzlich mit „Entity-Zentriertheit“ (Satzgegenstand) verknüpft (ebd.). ${ }^{84}$ Vogel (ebd., 237) führt zudem aus, dass die Markierung von sekundärer Thetizität durch ein es im Vorfeld auch kategorische Sätze wie (127) erfasst. In diesem Sinne wäre also die Funktion des Vorfeld-es eine doppelte, nämlich Rhematisierung und Markierung von (sekundärer) Thetizität. An (127) ist außerdem zu sehen, dass die Simulierung des Erstaktanten nur positional erfolgt, denn es gibt im Satz ein nicht-simuliertes, ,echtes“ Subjekt im Mittelfeld (,ein Gottseliger Man“).

Subjektlose Passivsätze mit Vorfeld-es stellen einen sozusagen noch perfekteren Fall der positionalen Valenzsimultion dar, da sie kein „echtes“ Subjekt enthalten. Sie haben trotzdem insofern einen anderen Status als (127), als das Verb hier nur in der 3.Ps.Sg.-Form stehen kann:

Es wurde auch, wenn von der Volkspolizei gesprochen wurde, nicht von den 'Bullen' gesprochen. (21) (SB)

Die Korrespondenz zwischen es und dem Finitum ist hier nur eine „Scheinkorrespondenz“, denn bei anderer Vorfeldbesetzung muss das es verschwinden, was für „normale“ Subjekte nicht gilt. Daher gehe ich hier in Anlehnung an Zifonun (1995: 44) von einem Standardwert aus, der die 3.Ps.Sg. ist und der durch ein zusätzliches, (notgedrungen) korrespondierendes Vorfeld-es ergänzt werden kann, wenn das Vorfeld nicht anderwertig besetzt wird. Subjektlose Passivsätze unterscheiden sich von subjekthaltigen, nicht impersonalen aktivischen (oder auch passivischen) Sätzen des Weiteren auch darin, dass sie „eventzentraler" sind (vgl. dazu Vogel 2006: 226, 234). Trotz der Differenzen zwischen aktivischen und passivischen Sätzen mit Vorfeld-es kann festgehalten werden, dass sie unter dem Aspekt der Valenzsimulation doch auf einen gemeinsamen Nenner zu bringen sind.

Im Falle von Sätzen wie (128)-(132) gehe ich mit Ágel (2000b: 230) und entsprechend den Ausführungen oben davon aus, dass diese eine INSP-Simulation aufweisen. Auch diese Gruppe ist heterogen und muss weiter ausdifferenziert werden. Zunächst werden die

\footnotetext{
${ }^{84} \mathrm{Vgl}$. hierzu auch die Unterscheidung von Geschehens- und Handlungsperspektive in Welke (2002).
} 
einzelnen es-Vorkommen dargestellt, dann wird die Frage nach der Konstruktionalität und somit auch der Begriff konstruktionelle Valenzsimulation geklärt.

Experiencer-Impersonalia können mit (s. (128)) oder ohne es stehen, vgl:

(134) $[\ldots]$ den ich wuste selber nicht, wie mir war, wen ich auff der gaßen gieng [...] (36) $(\mathrm{NE})^{85}$

Sie zeichnen sich dadurch aus, dass sie primäre Thetizität darstellen (Vogel 2006: 225) und kein persönliches grammatisches Subjekt enthalten. Zunächst erscheint im Mittelhochdeutschen das Vorfeld-es in solchen Konstruktionen ${ }^{86}$ (Vogel 2006: 194), später, in der neuhochdeutschen Periode, wo dann der Prozess der Subjektivierung den ganzen Bereich der Thetizität erfasst, wird ein formales Subjekt-es eingefügt, das auch im Mittelfeld erscheinen kann, ja in bestimmten Fällen tendenziell zunehmend sogar muss (Wegener 1999: 192). Ágel (2000a: 1872) bezeichnet diesen Prozess als Umkodierung. Dabei können im Deutschen zwei Typen von Umkodierungen unterschieden werden (ebd.): 1. Der Zweitaktant wird nominativiert, vgl. mir fehlt + Gen $\rightarrow$ mir fehlt + Nom; 2. Der akkusativische / dativische Erstaktant wird nominativiert, vgl. mich friert $\rightarrow$ ich friere. Ein wichtiger Vorgang ist hierbei die Einführung von Formalsubjekten (Seefranz-Montag 1995: 1279), was „die formale Anpassung des Satzmusters an den 1. Umkodierungstyp“ bedeutet (Ágel 2000a: 1872). So erscheint es in diesen Experiencer-Konstruktionen. Zu seiner Reinterpretation als Nominativ trägt die Veränderung (Vereinfachung) in seiner Morphologie bei: der Genitivschwund in seiner Flexion. Es handelt sich dabei um „den Zusammenfall der mittelhochdeutschen (mhd.) Laute $z$ und $s$ und die damit erfolgte Neutralisierung von Gen. es und Nom./Akk. ez“" (Ebert 1978: 55f., vgl. auch Polenz 2000: $154)$.

Im Gegensatz zum teils fakultativen es bei Experiencer-Impersonalia ist das es in (129) bis (132) obligatorisch. Diese Obligatorik des es-Vorkommens entspricht der in der Fachliteratur bekannten sprachhistorischen Tendenz zur Zweigliedrigkeit bzw. (wenn auch scheinbaren) Subjekthaltigkeit deutscher Sätze. Die Zunahme im Gebrauch des fixen es zuerst in impersonalen Konstruktionen wie es regnet ist seit althochdeutscher Zeit festzustellen (Ebert 1978: 55). Den Grund dafür sieht man gewöhnlich in der Tendenz zur systematischen Zweiteilung des Satzes in Subjekt und Prädikat (ebd., 53). In Anlehnung an

${ }^{85} \mathrm{Zu}$ weiteren Beispielen mit anderen Verben (mich friert usw.) s. Seefranz-Montag (1995) und Vogel (2006).

${ }^{86}$ Dies würde dort eine positionale Simulation bedeuten. 
Faucher (1996: 38f.) würde ich jedoch die Ansicht vertreten, dass nicht das Schema VerbSubjekt ausschlaggebend ist. „Zwingend geworden ist vielmehr die Implikatur: wenn konjugiertes Verb, dann auch ein numerus-kompatibler Nominativ, (der u.U. eine Subjektfunktion markieren kann, aber nicht muss)“. Dafür spricht m.E. auch, dass bei Infinitiven es fehlen konnte (vgl. Behaghel 1928: 450). Den Gebrauch eines fix werdenden, simulierenden es auch bei anderen Valenzträgern, nämlich so genannten okkasionellen Ereignisverben (s. (130), vgl. Näßl 1996), findet man in Ansätzen schon im Althochdeutschen (Näß1 1996: 233). Okkasionelle Ereignisverben sind Konstruktionen mit subjektgeneralisierendem es, die „im allgemeinen ein bestimmtes Subjekt bei sich haben, also habituell ,persönlich“ konstruiert werden, aber mit dem Scheinsubjekt es in unpersönlicher aktivischer Konstruktion erscheinen können“ (ebd., 36). Eine Ausdehnung dieser „Strategie“ auf Verben, die unterscheidlichen Wortfeldern zuzuordnen sind (ebd., 91), erfolgt allerdings erst ab mittelhochdeutscher und setzt sich fort in neuhochdeutscher Zeit. Ein weiteres Beispiel hierfür ist (135):

[...] endlich hörede etwas gantz dunckel bochen, ich lag aber stille, es bocht noch 2 oder 3 mal, so dachte ich, es were ein gespenst, endlich als es nicht wolte auff hören, und recht starck anschlug, so kroch ich heraus [...] (26) (NE)

Dabei ergeben sich Veränderungen hinsichtlich der von der Valenzsimulation betroffenen Verben, bspw. wird die im mittelhochdeutschen verbreitete Gruppe von Bewegungen, Lichterscheinungen und akustische Phänomene ausdrückenden Verben (ebd., 249) um weitere (u.a. Geruchsverben, Emotionsverben) ergänzt, ja sogar so weitergeführt, dass auch Valenzträger mit menschlichem Subjekt erfasst werden können (ebd., 253). Begünstigt wird dieser Prozess m.E. auch dadurch, dass selbst beim phorischen es grammatische Restriktionen im Genus- ((136)) und Numerusbereich ((137)), die die Korrespondenz zwischen es und dem jeweiligen Bezugsglied betreffen, abgebaut werden können (vgl. hierzu auch die nicht kongruierenden Fälle in Kapitel 2.4):

(136) und merckte gleich darbeÿ, das ich noch nicht recht durchreiniget were, das ich im ofen des Elends noch beßer müste bewehret werden. doch hörete ich diesen schönen tohn noch lange, aber es verlohr sich immer weitter, ich kans aber itzo nicht recht beschreiben wie dieses so wunderschön (35) (NE) 

kleider, und gingen hin und verborgen es [...] (45f.) (NE)

In (136) haben wir es unter funktionalem Gesichtspunkt mit dem phorischen „Normaltyp“ zu tun, aber in grammatischer Hinsicht wird die Genuskorrespondenz (tohn - Maskulinum) aufgehoben. Der Grund dafür dürfte sein, dass der Autor dadurch auch mit dem schönen thon verbundene Gefühle und Eindrücke zum Ausdruck bringen, mitmeinen kann. Diese Art von Generalisierung des Subjekts wird in der Fortführung der Textstelle durch die beiden dieses beibehalten:

$$
\begin{aligned}
& {[\ldots] \text { ich kans aber itzo nicht recht beschreiben wie dieses so wunderschön, mit }} \\
& \text { meinen leibligen augen und ohren hörete ich dieses nicht, sondern nach dem inner } \\
& \text { geiste [...] (35) (NE) }
\end{aligned}
$$

In (137) finden wir zwar kein Subjekt, sondern ein Objekt-es, aber aus dem Beispiel ist ersichtlich, dass durch es (statt etwa sie) eine Generalisierung stattfinden kann.

Bezüglich der Impersonalia und der okkasionellen Eregnisverben lässt sich zusammenfassend sagen, dass durch die es-Valenzsimulation die primäre Thetizität der Impersonalia ausgedehnt wird auf einen sekundär thetischen Bereich, wo (u.a.) $)^{87}$ okkasionelle Ereignisverben entstehen.

Bevor ich auf den gemeinsamen Nenner (konstruktionelle Simulation) der Belege (128)(132) eingehe, sollen (131) und (132) aus dieser Gruppe noch kurz kommentiert werden. (131) ist ein Beispiel für das „Präsentativ-Syntagma“ es gibt (Weinrich 1993: 398). Dieser Fall muss deswegen getrennt behandelt werden, weil er historisch später entsteht ${ }^{88}$ und weder zu den primären Impersonalia noch zu den okkasionellen Ereignisverben gehört. Es ist eine feste unpersönliche Wendung (Näß1 1996: 32), deren Entstehen mit der Grammatikalisierung des Verbs geben in Zusammenhang gebracht wird (Lenz 2007). Trotzdem ist auch hier die oben bereits beschriebene Generalisierung von es sichtbar, vgl. den Beleg ,[...] wann man Pulver auf die Pfanne schüttet, und die Luft aufsetzt, so gibt es einen grossen Knall [...]“ bei Ebert (1993: 347). In diesem Beispiel ist der phorische Charakter noch erkennbar: „geben wurde in seiner ursprünglichen Bedeutung erzeugen,

\footnotetext{
${ }^{87}$ Vgl. hierzu Vogel (2006: 224).

${ }^{88}$ Das früheste häufigere Auftreten von es gibt wird ins 16. Jh. datiert (Newmann 1998: 307), aber ,,erst in neuhochdeutscher Zeit ist die Bedeutung zur generischen Existenzaussage hin ausgeweitet.“ (Lenz 2007: 63f.)
} 
hervorbringen verwendet, es bezog sich zuerst als reine Anapher auf den sprachlichen Kontext“"(Scheibl 2000: 376, vgl. auch das Beispiel ebd.).

Der simulierte Zweitaktant in (132) ist insofern an dieser Stelle zu behandeln, als hier auch Generalisierung vorliegt, wie bei Subjekte simulierenden es-Vorkommen, die keine konkreten oder persönlichen Subjekte darstellen (vgl. Impersonalia und okkasionelle Ereignisverben oben). Das Verb meinen mit fixem Objekt-es ist laut Digitaler Grimm (Lemma meinen, Bedeutung 5a) „seit alters bis auf heute sehr gewöhnlich“, es wird dort ein althochdeutsches Beispiel von Notker angeführt. In der Fachliteratur findet sich allerdings kein Hinweis darauf, dass man es auch beim fixen Objekt-es etwa mit einem produktiven Muster wie bei okkasionellen Ereignisverben zu tun hätte.

An dieser Stelle komme ich auf den Begriff der konstruktionellen Simulation zurück. Wir haben gesehen, dass die Belege (128) bis (132) sich hinsichtlich zahlreicher Merkmale (Syntax, Semantik, Geschichte) unterscheiden. Abgesehen von (132) haben sie aber einen gemeinsamen Nenner: die Verbform 3.Ps.Sg. Diese Kategorie wird teils fakultativ (Experiencer-Impersonalia, (128)), teils obligatorisch (vgl. (129)-(131)) um ein (valenz)simulierendes es erweitert. In Anlehnung an Ágel (2003: 21f.) kann man hier davon ausgehen, dass hier „sekundär paradigmatisierte Valenzträger“ entstehen. Das bedeutet, dass die betroffenen Verben zwar Flexionsformen der ursprünglichen Verben sind, aber zugleich eine Flexionsform haben, die obligatorisch die 3.Ps.Sg. ist, sich nicht ändern kann und daher hier ein sekundäres Paradigma zum jeweiligen Verb angenommen werden kann. Solche sekundären Paradigmen sind eindeutig an eine bestimmte Semantik gebunden, nämlich an eine Geschehenssemantik oder Thetizität. Die Fügung [3.Ps.Sg. $\left.+(e s)^{89}\right]$ mit Thetizitätsmarkierung ist somit eine besondere Verbindung von Form und Bedeutung, wobei die morphosyntaktischen und semantischen Besonderheiten nicht aus den Eigenschaften der einzelnen Bestandteile der Fügung abzuleiten sind, die klassische Definition von constructions (im Weiteren Konstruktionen) in der Konstruktionsgrammatik, vgl. Fillmore (1989: 20) und Fillmore / Kay / O’Connor (1988: 504). Bei Beispielen dieser Art wird in der vorliegenden Arbeit davon ausgegangen, dass die Valenzsimulation dieser es-Typen konstruktionell bedingt ist. Dementsprechend wird von konstruktioneller Valenzsimulation gesprochen. Diese Art von Valenzsimulation soll in 3.3 näher beschrieben werden.

\footnotetext{
${ }^{89}$ Die Klammern weisen auf die Fakultativität bei Experiencer-Impersonalia hin.
} 
Zum historischen Einwand bezüglich der Grammatikalisierung der Valenzsimulation (s. oben bzw. Ágel 2000: 230) lässt sich nun auf Grund der Fachliteratur (s. oben) Folgendes sagen:

- Primäre Impersonalia (Typ: es regnet) sind seit althochdeutscher Zeit belegt und sind am ältesten.

- Okkasionelle Ereignisverben erscheinen zuerst im Althochdeutschen, kommen häufiger aber erst ab mittelhochdeutscher Zeit vor.

- Das Vorfeld-es erscheint im Mittelhochdeutschen.

- Experiencer-Impersonalia erscheinen zunächst mit Vorfeld-es im Mittelhochdeutschen, „bekommen“ ihr konstruktionelles es aber erst später und werden in der neuhochdeutschen Periode umstrukturiert.

Möglich ist eine Entwicklung, während der die primären Impersonalia als Muster dienen, zu denen analog okkasionelle Ereignisverben gebildet werden. Die Grammatikalisierung würde in diesem Sinne innerhalb der Gruppe der okkasionellen Ereignisverben stattfinden, indem immer mehr verbale Wortfelder an diesem Prozess beteiligt sind, also die Generalisierung durch es auf andere Kontexte ausgeweitet wird, ein typischer Zug von

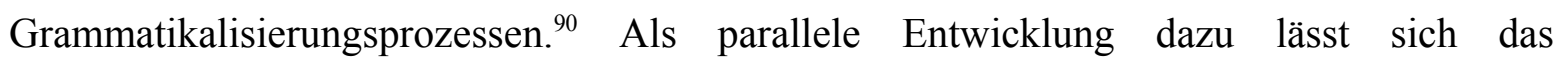
Aufkommen des Vorfeld-es als sekundärer Thetizitätsmarker betrachten, vgl. dazu Vogel (2006). Die Experiencer-Impersonalia ließen sich auf diese Weise ins System integrieren, da sie als primär thetische Fügungen zuerst ein zusätzliches Vorfeld-es bekommen, das entsprechend der zunehmenden (formalen) Subjektivierung deutscher Sätze umgedeutet wird.

Bevor auf die angesprochenen Konstruktionen eigegangen wird, soll erläutert werden, wie das in 2.3 behandelte Korrelat-es sich in die Gruppe von Valenzsimulationen fügt und somit als eine Übergangsform zwischen phorischem und nicht-phorischem es angesehen werden kann.

\footnotetext{
${ }^{90} \mathrm{Zu}$ berücksichtigen wären hier dann so genannte „context types“ (Diewald 2006), insbesondere für den Anfang und die Verbreitung wichtige „untypical“ und „,critical contexts“" (ebd. 4).
} 


\subsection{Das Korrelat-es als ein Fall von Valenzsimulation}

Im Folgenden soll gezeigt werden, dass das Korrelat-es als ein Fall von Valenzsimulation interpretiert und somit in das in 3.1 dargestellte System integriert werden kann und als Übergang vom phorischen zum simulierenden es anzusehen ist. In einem ersten Schritt soll die grammatische Argumentation erfolgen, im Anschluss daran wird auf die sprachhistorischen Aspekte eingegangen.

Zunächst sollen hier folgende Beispiele angeführt werden:

(139) ach meine liebe Mutter hat grose liebe und sorge vor mich, ach ich kan es dem lieben Gott und ihr nicht gnug dancken. (4) (NE)

(140) Endlich trug sichs zu das ich nach Eisenach reisete zu meinem Bruder (2) (NE)

Der Unterschied zwischen (139) und (140) ist in erster Linie formaler Natur. Beide stellen zwar einen pro-propositionalen Bezug her, leisten dies jedoch in unterschiedlicher Weise. Das es in (139) ist anaphorisch, das in (140) (gebunden) kataphorisch, wobei die Bezugnahme in (140) - gegenüber (139) - in einer festen Hauptsatz-Nebensatz-Struktur erfolgt. Genau das ist der Grund dafür, dass Zifonun (1995 und 2001) hier nicht mehr von Phorik spricht und in der vorliegenden Arbeit von gebundener Phorik die Rede ist. Hinzu kommt allerdings noch folgendes Problem: $\mathrm{Ob}$ wir von einem Korrelat und damit von einem strukturellen Verweis ausgehen oder ein phorisches, verallgemeinernd-summierendes, propositionales es (Admoni 1976) annehmen, dürfte vielfach von der Integriertheit des Nebensatzes abhängen, vgl. hierzu Kapitel 2.3.3. Das Korrelat-es kann in diesem Sinne als Übergang vom phorischen zum nicht-phorischen es angesehen werden, indem die Annahme eines Korrelat-es von der grammatischen Verfestigung der beteiligten Propositionen abhängt, also davon, ob bzw. in welchem (syntaktischen) Maße die grammatikalisierte Hauptsatz-Nebensatz-Struktur vorliegt oder nicht. Als Übergangsfall lässt sich das Korrelates somit auch als ein Fall von Valenzsimulation betrachten. Den Überlegungen in 2.3 folgend wird hier davon ausgegangen, dass das es kein selbständiger Aktant ist, sondern zusammen mit dem Nebensatz ein Diskontinuum bildet. Es simuliert die Erst- oder Zweitaktantenrealisierung sowohl syntaktisch - indem es die nebensatzförmige Ergänzung im Hauptsatz vertritt und auf sie strukturell verweist - als auch semantisch (Simulierung der semantischen Rolle); die semantische Rolle bekommt der korrespondierende Nebensatz 
zugeschrieben. Das Korrelat-es als rein struktureller Verweis und als Valenzsimulation stellt somit den Übergang vom phorischen zum nicht-phorischen es-Typ dar.

Der Übergangscharakter des Korrelat-es und sein Status als Valenzsimulation kann auch durch Beispiele mit es gilt und es heißt bestätigt werden:

\section{Nun gilt es, Zeit zu gewinnen. ${ }^{91}$}

[...] da war recht in mir wirklich und empfindlich, wen es heist In Weinstock Jesu stehen wir, gepfropft, und gantz mit Gott vereinet [...] (12) (NE)

Diese Belege erinnern an Korrelat-Fälle. Interessant ist dabei jedoch, dass das Korrelat-es fix (obligatorisch) geworden ist. Dies lässt sich nachweisen, wenn man den Nebensatz an den Anfang bewegt (die bekannte Umstellprobe bei Korrelaten) und dabei es nicht verschwinden darf. Das Auftreten dieses obligatorischen „Korrelats“ bzw. seine Verbindung mit gelten und heißen verursacht eine Bedeutungsänderung (sowie Lexikalisierung) und somit eine Umstrukturierung im syntaktischen Umfeld der Verben (Zitterbart 2002b: 166): Die geforderten Ergänzungen sind Verbativergänzungen und keine Subjektsätze; wir haben es auch hier, wie z.B. bei okkasionellen Ereignisverben, mit einer sekundären Paradigmatisierung der beteiligten Verben (Valenzträger) zu tun. Ein wichtiges morphologisches Merkmal, das für eine Interpretation dieser Konstruktion als Valenzsimulation spricht, ist weiterhin, dass der verbale Teil bezüglich Numerus und Person unveränderlich, d.h. immer 3. Person Singular, bleibt, was ein Charakteristikum von impersonalen Konstruktionen (also von Valenzsimulationen) ist.

Der Übergangsstatus wird auch historisch untermauert, indem man - wie oben bereits erwähnt - in der einschlägigen Fachliteratur davon ausgeht, dass das es impersonale, darunter auch das Vorfeld-es, auf das Korrelat-es zurückgeht:

Die oberste Bedingung für das Aufkommen des Impersonalien- 'es' waren Sätze mit es, il, in denen dieses Pronomen als Subjektswort rededeiktisch einen Vorstellungsinhalt vertritt, der zunächst dem Bewußtsein nur vorschwebend, hinterdrein in der Form eines abhängigen Satzes (meistens eines konjunktionalen Nebensatzes) oder einer infinitivischen Wendung zur Aussprache kommt [...] (Brugmann 1917: 15)

\footnotetext{
${ }^{91}$ Beispiel in Faucher (1996: 38).
} 
Stellt man die bisher beschriebenen Fälle der Valenzsimulation den phorischen Bezügen gegenüber, so ist ein struktureller Vergleich einerseits zwischen Bezügen auf Phrasen und Beispielen wie etwa (129) und (130), andererseits zwischen solchen auf Propositionen und Beispielen wie etwa (139) und (140) möglich. Dementsprechend kann von der Simulierung phrasalen Bezuges auf der einen und propositionalen Bezuges auf der anderen Seite gesprochen werden. Das binäre Valenzsimulationsbild kann weiterdifferenziert werden, sodass unter Beibehaltung der positionalen Valenzsimulation entsprechend den Überlegungen zum Korrelat-es innerhalb des konstruktionellen Zweigs zwei Subtypen postuliert werden:

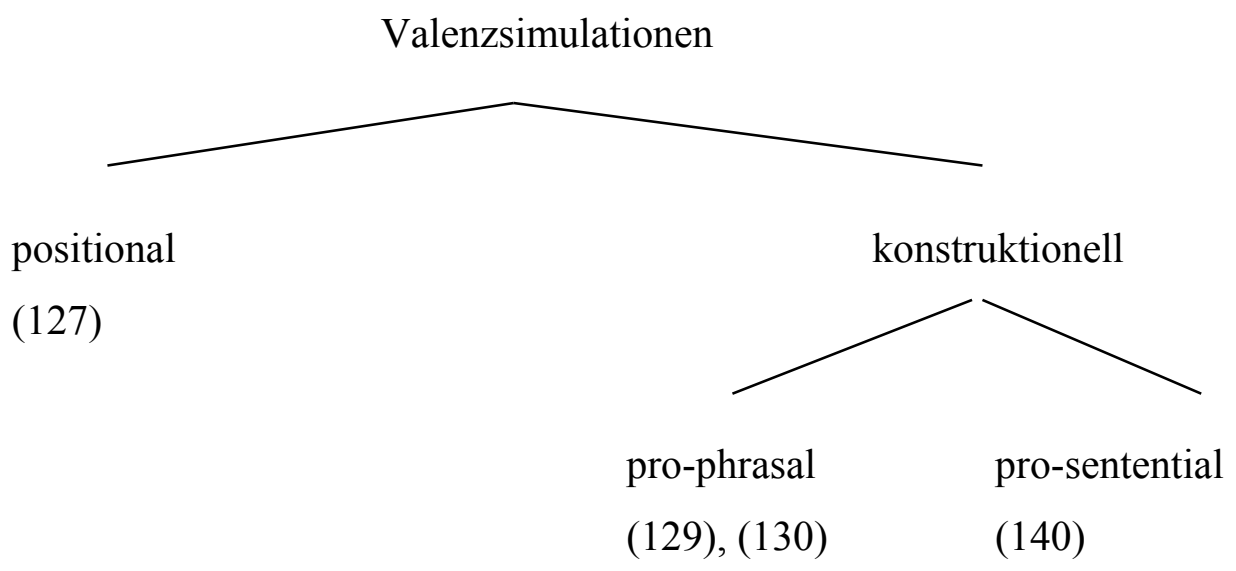

Die Valenzsimulation in (82) wird also pro-phrasal genannt, weil dieses es-Vorkommen als simulierender Erst- bzw. Zweitaktant Bezugnahmen auf Phrasen (in erster Linie Nominalphrasen) simuliert. Das Korrelat-es stellt eine pro-sententiale Valenzsimulation dar, indem hier die syntaktisch gebundene Version des pro-propositionalen Bezugs in (139) angenommen wird.

\section{3 es-Konstruktionen}

In diesem Kapitel wird der Versuch unternommen, einige Ideen und Konzepte der Konstruktionsgrammatik auf die Beschreibung des deutschen es zu übertragen. Die Konstruktionsgrammatik entstand in den 80er Jahren im Umfeld generativistisch orientierter, wortzentrierter und regelbasierter Grammatiktheorien. Als Begründer können 
Charles Fillmore und Paul Kay (Fillmore/Kay/O’Connor 1988) angesehen werden, andere prominente Vertreter dieser Theorie sind des Weiteren Adele E. Goldberg (Goldberg 1995) und William Croft (Croft 2002) ${ }^{92}$. Inzwischen sind auch in der germanistischen Linguistik zahlreiche Arbeiten veröffentlicht worden, die in konstruktionellem Rahmen enstanden sind, vgl. etwa die Sammelbände Fischer/Stefanowitsch (2006, 2008), Günthner/Imo (2006), die Dissertation von Imo (2007a) oder das ZGL-Sonderheft (2009).

Die bedeutendste der konstruktionsgrammatischen Prämissen ist wohl die These, dass Sprache und besonders Grammatik nicht durch den ständigen Gebrauch von Regeln, sondern in so genannten constructions erworben, gespeichert und produziert wird. Constructions sind

\section{$[\ldots]$ structures whose properties do not, in any obvious or familiar way ,follow from' what can be independently known about their constituent elements (Fillmore 1989: 20), [...] things which are larger than words, which are like words in that they have to be learned separately as individual whole facts about pieces of the language, but which also have grammatical structure [...] (Fillmore/Kay/O’Connor 1988: 504).}

Wie aus den obigen Zitaten ersichtlich, sind constructions (meistens ${ }^{93}$ ) polylexikalische Einheiten, die zwar strukturiert, aber nicht strikt kompositionell sind. Als zentrale Einheiten unseres sprachlichen (und grammatischen) Wissens werden sie ganzheitlich gespeichert und abgerufen. Das heißt jedoch nicht - und dies kommt auch im zweiten Zitat zum Ausdruck -, dass syntaktische Konstruiertheit aufgehoben wird, vgl. Fillmore (1989: 19) und Ágel (2003: 28). Die Idee, dass constructions bzw. Mehrworteinheiten Wörtern gleichgestellt werden sollten und von weitaus größerer Bedeutung sind als früher angenommen, finden wir, wenn auch mit unterschiedlichen Akzentuierungen, auch in anderen Theorien jüngeren Datums (Feilke 1996, 1998, Ágel 2003, 2004, hierzu s. weiter unten). Die Grundlagen der Konstruktionsgrammatik stehen mit neuesten humanethologischen und psycholinguistischen Erkenntnissen im Einklang. Diese legen nämlich nahe, dass bei Spracherwerb und -produktion nicht ein bereits vorhandener Regelapparat wiederholt durchlaufen wird und dass sprachliche Einheiten nicht das Ergebnis von Satzerzeugungsprozessen sind (Stein

\footnotetext{
${ }^{22}$ S. auch Fillmore (1989), Hopper (2000), Kálmán (2001) und Michaelis/Ruppenhofer (2001).

${ }^{93}$ Die Einschränkung gilt der von Goldberg (1995: 4) und Croft (2002: 17) vertretenen Ansicht, dass z.B. auch Morpheme als constructions betrachtet werden können, s. dazu weiter unten.
} 
1995: 14). In der Humanethologie heißt das: „Das Kind eignet sich nicht Regeln, sondern Beispiele, und zwar meistens fehlerhafte Beispiele an. Es ist dann imstande, auf Grund dieser Beispiele angemessene sprachliche Ausdrücke $\mathrm{zu}$ bilden [Übersetzung von mir, D.C.]“ (Csányi 1999: 239f.). Auch Befunde in der Spracherwerbstheorie bestätigen diese Einsicht:

Der Eindruck der Leichtigkeit, Schnelligkeit und weitgehenden Fehlerhaftigkeit des Spracherwerbs ist [...] nicht das Resultat produktiven Regelgebrauchs [...], sondern ein Ergebnis der Tatsache, dass das Kind sehr konservativ vorgeht und ganze Einheiten aus dem Input übernimmt [...] (Behrens 1999: 43f.).

Dass unter Annahme von Vorgeformtheit und fertigen sprachlichen Ausdrücken der für Sprache so wichtige Kreativitätsaspekt nicht zu kurz kommt, zeigt z.B. Imo (2007b) in Anlehnung an Croft (2002).

Im Folgenden wird der Versuch unternommen, grundlegende Feststellungen und Annahmen der Konstruktionsgrammatik bzw. mit ihr verwandter Theorien auf die Beschreibung deutscher es-Vorkommen anzuwenden. Von einer solchen Herangehensweise erhoffe ich mir neue Einblicke in dieses in der grammatischen Fachliteratur oft behandelte Phänomen. Die Forschung zu Konstruktionen und polylexikalischen Strukturen liefert Ergebnisse, die auch in der es-Forschung genutzt werden könnten. Auf den ersten Blick scheint dies zumindest bei Fällen wie es regnet/es gibt usw. - also bei Impersonalia - auf der Hand zu liegen, da hier wahrscheinlich jeder sagen würde, dass diese feste, polylexikalische Verbindungen darstellen. In diesem Sinne ist Ágels Kritik (2004: 65) an der Phraseologieforschung, solche es-Vorkommen nicht $\mathrm{zu}$ berücksichtigen, m.E. berechtigt. Wie wir sehen werden, können auch phorische es-Verwendungen über Eigenschaften von Konstruktionen verfügen.

\subsubsection{Konstruktionsgrammatik und verwandte Theorien}

Die Idee der Konstruktionsgrammatik, dass es in der Grammatik Mehrworteinheiten mit kompositionell nicht prädiktabler Form und/oder Bedeutung gibt, und dass diesen in Sprachproduktion, -rezeption und -erwerb eine sehr wichtige Rolle zukommt, finden wir 
auch in anderen Theorien vor, die hier ohne Anspruch auf Vollständigkeit dargestellt werden sollen.

Bereits bei Karl Bühler finden wir interessante Überlegungen, die etwas mit den Grundannahmen der heutigen Konstruktionsgrammatik zu tun haben dürften. Bühler (1965: 252f.) berichtet über ein kleines Experiment, bei dem er so genannte ,syntaktische Schemata" entdeckt haben will:

Sie [die Versuchspersonen: Psychologen und Studenten, D.C.] hatten epigrammatisch geschliffene Sentenzen, die ich jedem von ihnen einzeln vorlas, rasch zu verstehen und, soweit es sich gab, kritisch zum Inhalt eine Stellungnahme zu gewinnen. [...] Und immer wieder wurde dann beschrieben, daß dies oder jenes ganz oder teilweise leere syntaktische Schema [Hervorhebung im Original, D.C.] der eigentlichen Formulierung einer Antwort vorherging und das faktische Sprechen irgendwie erkennbar steuerte.

Es scheint hier um Schemas zu gehen, die die sprachliche Rezeption steuern. Damit im Zusammenhang könnte man auch die so genannten „Denkmodelle“ erwähnen. Im Falle von transitiven Verben spricht nämlich Bühler (ebd., 251) vom Denkmodell der Handlung, das sich grammatisch als eine Fügung von [Nominativ + Verb + Akkusativ] manifestiert. Dies entspricht der transitiven Argumentstruktur (construction) bei Goldberg (1995). Mit Bezug auf unseren Untersuchungsgegenstand muss an dieser Stelle noch auf das Denkmodell der Impersonalia (ebd., 250, 375f.) aufmerksam gemacht werden, denn hierzu gehören auch Sätze mit es-Impersonale.

Stephan Steins Überlegungen $(1995,2004)$ zur „formelhaften Sprache“ beziehen sich in erster Linie auf gesprochensprachliche Phänomene. Stein (1995: 19) merkt jedoch an, dass in Untersuchungen zur formelhaften Sprache auch so genannte syntaktische „Ausdrucksmuster“ mit einbezogen werden sollten. Diese

syntaktischen Verfestigungen nehmen zum Teil selbst wiederum semantische oder pragmatische Funktionen wahr, die über den Verknüpfungscharakter hinausgehen und aus den Verknüpfungskonventionen allein nicht abzuleiten sind (ebd.).

Stein führt zur Untermauerung seiner These mehrere Beispiele an, z.B. verweist er auf eine spezifische (generische) Verwendung des bestimmten Artikels in Verbindung mit Personenbezeichnungen und erwähnt auch andere, Satzverknüpfungen steuernde Muster wie 
nicht nur - sondern auch; zwar - aber, vgl. hierzu auch Fillmore (1989: 23ff., dort mit englischen Beispielen). Im Grunde genommen bewegt er sich aber vor allem im Bereich „gesprächspezifischer Formeln“, die der Erleichterung der mündlichen Kommunikation dienen (2004: 279). Die Konstruktionsgrammatik geht hier weiter (vgl. Croft 2002) und bezieht Überlegungen wie die von Stein auch auf die „herkömmliche“ Grammatik.

Helmuth Feilkes Begriff Ausdruck bzw. seine Theorie der idiomatischen Prägung (1996, 1998) bestimmter Ausdrücke liefern sozusagen einen soziopragmatischen Hintergrund zur Konstruktionsgrammatik. Es handelt sich hier um eine ganz komplexe Sprachtheorie, die im Grunde genommen davon ausgeht, dass kompetente Sprecher und Hörer einer Sprache nicht nur entscheiden können, was geht, und was nicht geht, sondern vielmehr können sie auf Grund pragmatisch dominierter Präferenzen zwischen verschiedenen Möglichkeiten wählen. Dabei spielen Ausdrücke mit „kompositionell nicht prädiktabler, präferentieller Bedeutung“ (1998: 74) eine sehr wichtige Rolle. In der Tat ähnelt eine solche Definition denen von constructions in der Konstruktionsgrammatik. Dabei betont auch Feilke (ebd.), dass eine solche „Prägung“ sowohl die formale als auch die inhaltliche Seite von Ausdrücken betrifft. Damit ist eigentlich die grammatische Seite auch schon mit gemeint und tatsächlich spricht auch Feilke (1996: 241) von „syntaktischen Prägungen“, die er tabellarisch zusammenfasst. Wichtig ist an dieser Stelle auch auf das Zusammenspiel von Wörtern und Ausdrücken einzugehen: „Das Wort steht semantisch unter dem Zeichen des Ausdrucks“ (1998: 70), so Feilke. Er geht also davon aus, dass unter semantischem Aspekt der Ausdruck das Wort dominiert, grammatisch das Wort den Ausdruck (vgl. 1998: 77f. und Ágel 2003: 29). Genau diese Beziehung wird auch in der Konstruktionsgrammatik postuliert, bei Michaelis/Ruppenhofer (2001: 38) z.B. als das so genannte „override principle“: „If lexical and structural meanings conflict, the semantic specifications of the lexical element conform to those of the grammatical structure with which it is combined." Diese Annahme heißt zugleich, dass Konstruktionen eine eigene Grundsemantik zugeschrieben wird: „Syntactic frames are directly associated with semantics, independently of the verb which may occur in them." (Goldberg 1995: 19). An dises Konzept erinnern die Überlegungen bei Ickler (1990). Sie spricht davon, dass deutsche Verben in unterschiedliche Kasusrahmen (1990: 4) eingebettet werden können, wodurch die Bedeutung des Verbs (bei ihr: die „Modifikatorbedeutung“) gemäß dem jeweiligen Kasusrahmen (dessen Bedeutung bei ihr „Funktorbedeutung“ heißt) perspektiviert wird. Ickler (1990: 9f.) bringt zahlreiche Beispiele dafür, dass ein bestimmter Kasusrahmen eine 
bestimmte Funktorbedeutung mit einer bestimmten Perspektive vorgibt, die dann durch die Modifikatorbedeutung des jeweiligen Verbs spezifiziert wird. ${ }^{94}$ Die Beispiele zeigen außerdem, dass die jeweilige Perspektive oft durch bestimmte Formmerkmale gesteuert wird (z.B. die Applikativ genannte Perspektive durch das Präfix -be). Der Perspektivierungsbegriff Icklers erinnert an den von Welke (2002), wobei eine Perspektivierungspotenz bei Ickler nicht (nur) Wörtern, sondern auch Kasusrahmen zugeschrieben wird. Das ist ein wichtiger Unterschied, denn es wird angenommen, dass Wörter in Kasusrahmen perspektiviert werden. In eine konstruktionsgrammatische Terminologie übersetzt heißt dies: Konstruktionen („Kasusrahmen“) perspektivieren. Nimmt man z.B. den Applikativ, dem Welke ein ganzes Kapitel widmet (2002, Kapitel 6), so lässt sich sagen, dass die „Veränderungen der inhärenten semantischen Struktur der Verben“ (ebd., 329) nicht auf Wortebene erfolgen, sondern durch die deutsche ApplikativKonstruktion bewirkt werden, vgl. die Analyse der be-Verben durch Michaelis/Ruppenhofer (2001)

Auch wenn in den bisher kurz dargestellten Ansätzen wichtige Details unterschiedlich bewertet werden können und sollten, so kann die Fokussierung auf das Verhältnis zwischen Wörtern und Ausdrücken (Feilke) bzw. Kasusrahmen (Ickler) doch als gemeinsamer Nenner angesehen werden. Auf eben dieses Verhältnis und die daraus resultierenden theoretischen „Komplikationen“ wird auch in der Konstruktionsgrammatik großer Wert gelegt. Das Zusammenspiel von Wörtern und Konstruktionen bietet dabei der Konstruktionsgrammatik einen wichtigen Vorteil gegenüber manchen anderen Grammatiktheorien. Dieser Vorteil besteht in der Ökonomie. Goldberg (1995: 11f.) zeigt am Beispiel des englischen Verbs „kick“ in acht unterschiedlichen Kontexten, dass es nicht überzeugend ist, bei jeder Verwendung des Verbs von jeweils anderen, aus den unterschiedlichen Argumentstrukturen resultierenden Verbbedeutungen auszugehen:

Instead of positing a new sense every time a new syntactic configuration is encountered $[\ldots]$ a constructional approach requires that the issue of the interaction between verb meaning and constructional meaning be addressed (ebd., 12).

Es geht also vielmehr darum, dass Verben in jeweils anderen grammatischen Grundkonstellationen (Konstruktionen) auftreten können (z.B. transitive/ditransitive

${ }^{94}$ Im Gegensatz zum „override principle“ spricht Ickler (1990: 13) jedoch von „Interdependenz“ zwischen Verbsemantik und Funktorbedeutung des Kasusrahmens. 
Konstruktionen). Dabei bringt das Verb seine Valenzpotenz und die damit verbundene Verbsemantik (Modifikatorbedeutung) mit in die Valenzpotenz der Konstruktion und die damit verbundene Konstruktionsvalenz (Funktorbedeutung) hinein und spezifiert diese: „[...] the meaning of an expression is the result of integrating the meanings of the lexical items into the meanings of constructions“ (Goldberg 1995: 16). Michaelis/Ruppenhofer (2001: 39) verwenden in Anlehnung an Fillmore/Kay die beiden Ausdrücke „minimal valence“ (= Valenzpotenz des Verbs) und „fully specified verbal valence“ (= Valenzpotenz der Konstruktion). Um auf den eingangs erwähnten Kreativitätsaspekt (Imo 2007b) kurz zurückzukommen, könnte Kreativität gerade auch darin bestehen, dass Verben mit ihrer eigenen (lexikalischen) Semantik in Konstruktionen verwendet werden, wodurch diese Verben neue (konstruktions-)semantische Potenziale verliehen bekommen (vgl. Goldberg 1996: 30ff.).

Ágel (2003) z.B. wendet die Feilke'sche Theorie auf die valenzielle Beschreibung bestimmter Konstruktionen mit sitzen, Phraseologismen und labiler Verben (2003: 18) an. Dabei kommt er zum Schluss, dass nicht nur Wortvalenzträger, sondern auch Ausdrucksvalenzträger postuliert werden sollten. So ist z.B. agensresultativisches ist geschmolzen als Ausdrucksvalenzträger zwar ins Wortparadigma von schmelzen integriert, aber zugleich ist es auch bestimmend, wenn man entscheiden möchte, ob schmelzen als Wortvalenzträger primär rezessiv oder kausativ ist (2003: 31). Ein ähnlicher Fall liegt auch bei Medialkonstruktionen wie z.B. liest sich vor (2003: 21f.), wo die sich-Variante einen „sekundär paradigmatisierten VT [Valenzträger, D.C.]“ darstellt, vgl. hierzu Kapitel 3.1. Dementprechend stellen die einzelnen sich-liest-Formen (las sich; hätte sich gelesen usw.) zwar Flexionsformen von lesen dar, aber zugleich haben sie auch einen eigenen Ausdrucksvalenzträger sich liest als zu Grunde liegende sekundär paradigmatisierte Flexionsform. In die Konstruktionsgrammatik übersetzt: Es geht hier darum, dass es eine deutsche mediale sich-Konstruktion gibt, welche Verben, die in sie „hineintreten“, semantisch und auch grammatisch ${ }^{95}$ modifiziert. Die Konstruktionssemantik „Eigenschaftslesart ${ }^{\star 96}$ wird also durch die Verbbedeutung spezifiziert. Die von Ágel postulierte sekundär paradigmatisierte Valenz ist also im Sinne der Konstruktionsgrammatik nichts anderes, als eine durch die Konstruktion geprägte Valenz des Lexems, die immer

\footnotetext{
${ }^{95}$ Es muss in diesem Fall eine Modalbestimmung erscheinen.

${ }^{96}$ Es ist nur eine für den hiesigen Zweck erfundene und daher recht grobe Bezeichnung. Damit ist gemeint, dass Verben mit sich und Modalangabe Eigenschaften des im Satz auftretenden Subjekts ausdrücken.
} 
dann erscheint, wenn das Verb in die jeweilige Konstruktion eingebettet, oder anders formuliert: in ihr verwendet wird.

Nach dieser kurz gehaltenen Überschau von Theorieansätzen, die in irgendeiner Form die auch in der Konstruktionsgrammatik bedeutende Idee des kompositional nicht Erklärbaren in den Vordergrund stellen, sei noch auf die meiner Meinung nach ausgezeichneten Überlegungen Knoblochs im ZGL-Sonderheft Konstruktionsgrammatik (2009) hingewiesen. Knobloch (2009: 390) macht mit Recht darauf aufmerksam, dass die konstruktionsgrammatischen Grundkonzepte gar nicht neu sind:

Uralte fachliche Kontroversen scheinen in der KG wieder aufzuleben. Während Hermann Paul alle ausdrucksseitig komplexen sprachlichen Gebilde aus der

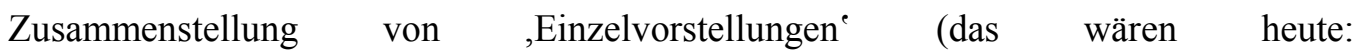
lexikalische/grammatische Elemente mit ihren Symbolbedeutungen) komponiert wissen wollte, zog der Psychologe Wilhelm Wundt eine Optik vor, die auf der Produktionsseite mit der Analyse einer ungegliederten,Gesamtvorstellung beginnt und mit deren strikt kompositioneller (einzelsprachlicher!) Resynthese endet.

Im Zusammenhang mit den wissenschaftshistorisch eindeutig belegbaren Parellelen zwischen Konstruktionsgrammatik und anderen Ansätzen formuliert Knobloch (ebd., 385, 387, 389) mehrfach seine Bedenken und argumentiert für einen vorsichtigen Umgang mit sprachlichen Daten in konstruktionellem Rahmen. Ausgehend von diesen Bedenken und angesichts der beinahe unüberschaubaren Fülle an konstruktionell geprägten Teiltheorien (vgl. hierzu u.a. Östman 2005, Feldman/Dodge/Bryant 2010 und Michaelis 2010, einen guten Überblick geben Fried/Östman 2004, Östman/Fried 2004 und Imo 2007a) erscheint es mir sinnvoll, bei der Awendung der Konstruktionsidee auf meinen Untersuchungsgegenstand den Rahmen möglichst eng zu halten. Dies führt dazu, dass praktisch nur die „Klassiker“ und für meine Zwecke gut umsetzbare Theoriebausteine (wie bspw. der Begriff formal idiom von Fillmore/Kay/ O’Connor 1988) herangezogen wurden. 


\subsubsection{Die [es+3.Ps.Sg.]-Konstruktion}

Es sind in erster Linie Sätze mit impersonalem es im Deutschen, die sich zu einer Analyse im Rahmen der Konstruktionsgrammatik anbieten. Die Konstruktionsgrammatik befasst sich nämlich vornehmlich mit Mehrworteinheiten, die in ihrer Form und Bedeutung fixiert sind. Dies trifft für ein es regnet oder es geht um sicherlich zu. Nicht zufällig kritisiert Ágel (2004: 65) die Phraseologieforschung, dass diese sich mit es-Impersonalia nicht auseinandersetzt. Wahrscheinlich aus dem Grunde nicht, weil sie in erster Linie an „extragrammatical idioms“ (Fillmore/Kay/O’Connor 1988: 505) interessiert sein dürfte, während das expletive es in „,zu puren“ „grammatical/formal idioms“ erscheint.

Als Grundlage sollen folgende Beispiele dienen:

(143) dan es donnert und hagelt, wen die Stuck abgingen (17) (BL)

(144) Ist bald alles erloschen vom Funken, so gehts heim zur Mutter zum Küchleessen (2) (SCHG)

(145) Es war doch erst März und noch ziemlich kalt (4) (SCHG)

(146) Alles mögliche dachte ich mir, es war mir Angst und bang dabei. (7) (SCHG) ${ }^{97}$

Gemeinsam ist all diesen Beispielen (vgl. aber auch die Belege (128) bis (131)), dass das Verb immer in der 3.Ps.Sg.-Form steht und meistens finden wir auch ein es. Diese 3.Ps.Sg. wird durch das Flexiv - $t$ realisiert. Eroms (2000: 188) spricht hier von einem Portmanteaumorphem, das neben Tempus und Modus auch „eine rudimentäre Personalform" beinhaltet. Den Valenzsimulationsgedanken wieder aufgreifend lässt sich hier sagen, dass in solchen Fällen die Erstaktantenstelle (gewöhnlich: das Subjekt) nur grammatisch nachgeahmt wird, ohne dass sie in der Valenzpotenz des Verbs in diesen Fällen enthalten wäre. Die Makrorealisierung (sprich: syntaktische Realisierung) des Erstaktanten ist es, die Mikroform (sprich: morphologische Realisierung) ist das Flexiv am Verb. ${ }^{98}$ In der Fachliteratur finden wir für die derartig gebrauchte 3.Ps.Sg. auch andere Termini: „die am wenigsten markierte Verbform“ (Jaeger 1992: 109) oder „Standardwert“ (Zifonun 1995: 44). Dies kann kein Zufall sein, die expletive 3.Ps.Sg. muss etw. Besonderes

${ }^{97}$ Weitere Beispiele mit Verben wie frieren und grauen in Eroms (2000: 189f.).

98 Erscheinen beide, so kann man in Anlehnung an László (1988) und Dürscheid (1995) von einem „Zweiebenenexpletivum“ sprechen. 
leisten. Das tut sie auch: Werden Verben in diesem „grammatischen Kontext“" verwendet, so handelt es sich dabei um den Ausdruck des Vorgangs/Geschehens/Zustands in unpersönlicher Weise. „Die Wahl von es als Subjekt erfolgt nicht zwangsläufig, sondern ist semantisch begründet“ (Eisenberg 2006: 176). Diese Aussage wird hier modifiziert, indem nicht die Wahl eines expletiven es als Subjekt in den Mittelpunkt gestellt, sondern angenommen wird, dass die Verbindung der 3.Ps.Sg.-Form des Verbs mit dem es zu einer Konstruktion führen kann, in der es nicht mehr pronominal gedeutet wird. Es wird mit Ágel (2009) davon ausgegangen, dass durch die Verbindung von es mit drittpersonigen Verbformen eine grundsätzlich offene Struktur entsteht. Die strukturelle Offenheit von [es + Verb $_{3 . P s . S g .]}$ führt dazu, dass persönliche und unpersönliche Deutungen möglich werden. Bei der unpersönlichen Bedeutung wird die 3.Ps.Sg.-Form sekundär paradigmatisiert (vgl. die Ausführungen hierzu in 3.1). Als einschlägige Beispiele kommen hier in erster Linie die so genannten okkasionellen Ereignisverben in Frage (Näß1 1996), vgl. etwa es klopft. Würde man hier annehmen, dass ein grundsätzlich expletives es Anwendung findet, so ist die Erklärung von Belegen dieses offenen Typs problematisch, denn hier sind im Prinzip persönliche und unpersönliche Deutungen möglich. Die unpersönliche Bedeutung entsteht „nicht durch die Realisierung eines expletiven es, sondern durch die schwache Interaktion des Ausdrucks $\left[=e s+\right.$ Verb $_{3 . P s . S g .}$, D.C.] mit dem Wortparadigma des Verbs (Wahl der dritten Person ohne Abwahl der ersten und zweiten) und des Personalpronomens (Wahl des es ohne Abwahl von er, sie).“ (Ágel 2009: 157) Im Folgenden soll diese spezifische Konstellation [es+3.Ps.Sg.]-Konstruktion genannt werden. Es ist eine Konstruktion, die nach der Terminologie in Fillmore/Kay/O'Connor (1988: 505) „grammatical“ und „formal/lexically opened idiom“ genannt wird. „Grammatical“ sind Konstruktionen, „,where verbs and noun phrases show up just where you would expect them.“ (ebd.) Das bedeutet, dass die in der Konstruktion enthaltenen Elemente an Stellen stehen und morphologisch aussehen wie herkömmliche Verbindungen von Verben und NPs, man vergleiche etwa es regnet mit es ( $\leftarrow$ das Baby) schläft. Lexikalisch offen und formal sind Konstruktionen folgender Art:

Formal idioms, by contrast, are syntactic patterns dedicated to semantic and pragmatic purposes not knowable from their form alone. It is the formal idioms which raise the most serious theoretical issues [...] (ebd., 505f.) 
Wichtig ist $\mathrm{zu}$ betonen, dass bei der in der vorliegenden Arbeit angenommenen [es+3.Ps.Sg.]-Konstruktion nicht nur das es, sondern auch die Einheitenkategorie ${ }^{99}$ 3.Ps.Sg. von Bedeutung, sogar wahrscheinlich primär ist. Das es kann nämlich bei ExperiencerImpersonalia (s. (146)) oft fehlen, aber auch anderswo ist dies nicht auszuschließen, vgl. Auers Beispiele für Verbspitzenstellung und fehlendes es in gesprochener Sprache (1993: 197). Die obligatorische Mikroform 3.PS.Sg. fehlt dagegen nie. Deshalb können wir hier auch von einer Morphemkonstruktion sprechen. Diese Auffassung ist konform mit der Ansicht, dass auch Morpheme Konstruktionen sein können (vgl. Fußnote 93). Natürlich stellt sich die Frage, warum dann in den meisten Fällen doch ein es erscheint. Dies ist eine typologische (und historische) Frage. Nach herkömmlicher Auffassung handelt es sich hierbei um eine Tendenz zur systematischen Zweiteilung des deutschen Satzes in Subjekt und Prädikat (s. etwa Ebert 1978: 53) und so erscheint es als „Scheinsubjekt“. Ähnlich argumentiert Eisenberg (2006: 177), der das Auftreten des es in solchen Fällen auf die Tendenz zurückführt, nach der jedes Verb ein Subjekt nehmen kann. Einleuchtend ist m.E. jedoch auch die Erklärung Fauchers (1996: 38f.), der die Einführung des es auf die „zwingende Verbform“ zurückführt. Wenn also konjugiertes Verb, dann auch ein kategorial regiertes Nominativelement, das nicht unbedingt wie ein herkömmliches Subjekt zu fungieren hat (vgl. 3.1). ${ }^{100}$

Bei Beispielen wie (144) oben geht es also darum, dass das jeweilige Lexem in die [es+3.Ps.Sg.]-Konstruktion eingesetzt wird, wodurch seine Semantik durch die der Konstruktion umperspektiviert wird. Es findet eine „Überführung in Vorgangsbedeutung“ statt (vgl. Welke 2002: 277). Damit dominiert die Konstruktion nicht nur semantisch, sondern auch grammatisch, denn die Verbform kann nur 3.Ps.Sg. sein, wenn die gewünschte Bedeutung erreicht werden soll. Die semantische Dominanz bedeutet Vorgangszentriertheit (dessen Merkmal Agensdezentrierung ist), die grammatische (morphosyntaktische) die 3.Ps.Sg. Schematisch kann man diesen Prozess wie folgt darstellen ${ }^{101}$ :

\footnotetext{
${ }^{99}$ Näheres zu diesem Begriff s. Eisenberg (2006: 17).

${ }^{100}$ Möglich ist auch die in der generativen Syntax favorisierte Lösung durch die Annahme von so genannten „empty categories/dummies“. Ohne darauf näher einzugehen, verweise ich diesbezüglich auf Eroms (2000: 190): „Wenn [...] in derartigen Konstruktionen materielle Elemente sogar vorfindbar sind [Hervorhebung von mir, D.C.], müssen diese genetisch erklärt, funktional gedeutet und syntaktisch geregelt werden.“

${ }^{101}$ Unter Lexem sind vor allem Verben zu verstehen, und natürlich können nicht alle Verben in eine solche Konstruktion eingeführt werden, es spielt dabei auch die Verbsemantik eine Rolle, vgl. das Prinzip der semantischen Kohärenz in Goldberg (1995: 50 und 2006: 40).
} 


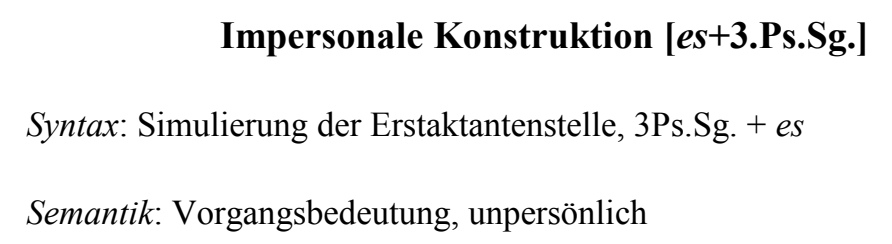

In diesem Zusammenhang soll darauf hingewiesen werden, dass die Tatsache, dass die Konstruktion semantisch (unpersönliche Bedutung) und grammatisch (sekundär paradigmatisierte 3.Ps.Sg.-Form des Verbs) auf ihre Bestandteile einwirkt, nicht bedeutet, dass diese Bestandteile grammatisch nicht beschrieben werden können. Ganz im Gegenteil: Es kann eine Arbeitsteilung zwischen Konstruktionsgrammatik und Valenztheorie angestrebt werden. Demnach liefert die Konstruktionsgrammatik eine Erklärung bestimmter nicht-phorischer es-Vorkommen sozusagen in top-down-Modus. So kann etwa im Falle von Belegen wie es klopft von einer impersonalen [es+3.Ps.Sg.]-Konstruktion mit Vorgangsbedeutung und dezentriertem Vorgangsträger die Rede sein, die neben der persönlichen eine zweite mögliche Variante der strukturell offenen Verbindung von es und einer drittpersonigen verbalen Wortform darstellt (vgl. Ágel 2009, s. oben). Dieses Gesamtprofil der Konstruktion färbt nun auf deren innere Morphosyntax ab, die wiederum in valenztheoretischem Rahmen beschrieben wird, indem gesagt wird, dass der Einfluss der Konstruktion etwa auf den Status von es $\mathrm{zu}$ dessen Interpretation als simuliertes Scheinsubjekt führt. An dieser Stelle soll auch darauf hingewiesen werden, dass eine Engführung valenztheoretischer und konstruktionsgrammatischer Überlegungen in der jüngeren Forschung angestrebt wird, vgl. Willems/Coen (2006), Jacobs (2009) und Welke (2009a, b), s. aber auch schon Hens (1996). Für die vorliegende Arbeit erweist sich meiner Meinung nach jedoch eine Strategie als sinnvoll, die die Anwendung von Valenz- und Konstruktionsgrammatik an klar bestimmten Stellen für klar bestimmte Aufgaben (s. oben) vorsieht. In diesem Sinne soll im Rahmen dieser Arbeit keine „konstruktionelle Valenztheorie“ (Willems/Coen 2006) entstehen.

Bei der Analyse der oben postulierten [es+3.Ps.Sg.]-Konstruktion können historisch gesehen die Witterungsverben (s. (143)) als Grundschema angesehen werden. ${ }^{102}$ Somit ist es auch möglich, eine Grundkonstruktion [es+Witterungsverb in 3.Ps.Sg.] anzunehmen, die dann als „syntaktisches Ausdrucksmodell“ (Ágel 2003: 28) dient. Diese Basiskonstruktion

\footnotetext{
${ }^{102}$ Beispiele wie die in (144) kommen erst seit dem späten Mittelhochdeutsch vor (Ebert 1978: 55ff).
} 
ist grundsätzlich vorgangsorientiert und stellt somit das Vorbild für die abgeleitete, analoge Variante [es+Verb in 3.Ps.Sg.] dar, die andere Verben umperspektivieren kann, s. etwa (144). Die Basiskonstruktion ist auch insofern der Ausgangspunkt, als die Verben hier konstruktionsgebunden sind und somit eine Grundperspektivierung darstellen, die in ihrer Bedeutung ,allgemeiner“ ist, was hier heißen soll, dass bei der [es+Witterungsverb in 3.Ps.Sg.]-Grundkonstruktion kein menschlicher Handelnder im Hintergrund anzunehmen ist. ${ }^{103}$ Verwendet man Verben wie das in (144) in der [es+3.Ps.Sg.]-Konstruktion, so entstehen die in 2.1 und 3.1 erwähnten okkasionellen Ereignisverben (vgl. auch (8) in 2.1). Hierzu liegt eine Monografie von Näß1 (1996) vor, in der diese Konstruktionen eingehend untersucht werden, wobei die einzelnen Fälle nach Wortfeldern geordnet werden. Zur Veranschaulichung sollen an dieser Stelle zwei Belege angeführt werden:

(147) Ich fing an zu beten und dann weinte ich so, dass es mich schüttelte, bis ich gerufen wurde. (8) (SCHG)

(148) Wer Zeit hatte, musste Obst auflesen; unter den Bäumen lag es dick von Obst (18) (SCHG)

In beiden Fällen findet man Verben vor, die persönlich gebraucht werden können, bei dem okkasionellen Gebrauch hier jedoch unpersönlich sind. In (148) ist zu sehen, dass es bei der Einsetzung der [es+3.Ps.Sg.]-Konstruktion hauptsächlich auf die Umperspektivierung ankommt, denn in (148) kommt Obst als mögliches grammatisches Subjekt in Frage. Dennoch wird es in eine PP ,ausgelagert“.

Außer Witterungs- und okkasionellen Ereignisverben können noch folgende Beispiele als Fälle für [es+3.Ps.Sg.]-Konstruktionen in Betracht gezogen werden:

(149) In Szeged lässt es sich sehr gut leben (im Sinne der Belege in Szatmári 1998)

(150) Gar lustig ist es, wenn wir über die Zukunft reden, mit der Mamka spricht sich 's so leicht Darüber... (1731) (LB)

(151) Es wurde im Kränzchen nicht gelesen u. nur geschwatzt; ich hätte lieber zu Hause gesessen. (106) $(\mathrm{SCH})$

${ }^{103}$ Croft (2002: 184) nimmt bei von einer Basiskonstruktion abgeleiteten verwandten Konstruktionen „lack of generality“ an. Das heißt, bei abgeleiteten Konstruktionen nimmt die Generalität der Ausgangskonstruktion ab, was mit Spezifizierungen einhergeht. 
Gemeinsam ist auch diesen Beispielen, dass die 3.Ps.Sg.-Form des Verbs gewählt wird und ein es auftritt. Hinsichtlich der Bedeutung kann man davon ausgehen, dass auch hier eine Perspektivierung vorliegt, die eventuelle Agentien in den Hintergrund rückt und dadurch der jeweilige Sachverhalt wie ein Geschehen oder Vorgang präsentiert wird. Es bietet sich daher an, auch hier die oben beschriebene [es+3.Ps.Sg.]-Konstruktion anzusetzen. Bei genauerem Hinsehen werden allerdings Differenzen sichtbar. In (149) haben wir es mit einer spezifischen Konstruktion (,specific“, vgl. Croft 2002: 17) zu tun, die sich aus der schematischen $\left[\right.$ sich+lassen + Verb $_{\text {trans }}$ Inf]-Konstruktion (,schematic“ im Sinne von Croft 2002: 17) und der [es+3.Ps.Sg.]-Konstruktion zusammensetzt. Durch diese Verbindung entsteht eine $\left[\text { sich }+ \text { lässt }+e+\text { Verb }_{\text {trans }} I n f\right]^{104}$-Konstruktion. Dabei addieren sich die beiden Konstruktionsbedeutungen, nämlich die modale der [sich+lassen+Verb ${ }_{\text {trans }}$ Inf]-Konstruktion und die der [es+3.Ps.Sg.]-Konstruktion. Dies kann wie folgt abgebildet werden:

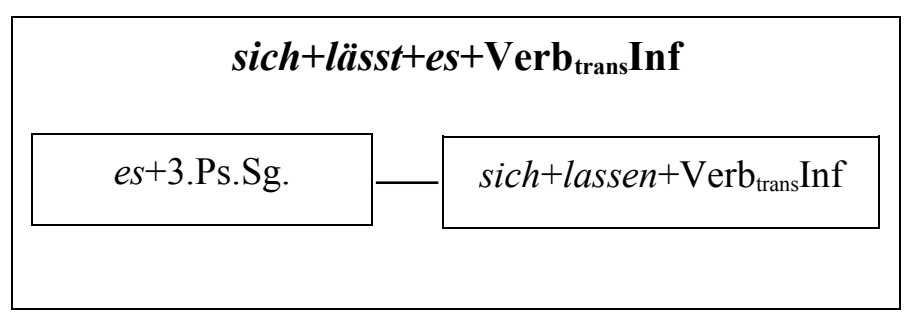

Mit Bezug auf (150) können wir ähnlich vorgehen, indem wir hier ebenfalls ein Zusammenspiel von zwei Konstruktionen annehmen. Die eine ist die in Kapitel 3.3.1 bereits erwähnte mediale [sich+Verb+Mod]-Konstruktion mit ihrer Eigenschaftslesart (vgl. die sekundäre Paradigmatisierung von lesen in liest sich und Fußnote 95 dort), die andere ist die [es+3.Ps.Sg.]-Konstruktion. Abgebildet werden könnte dies wie (149) oben. In (151) liegt ein Beispiel für das unpersönliche Passiv vor, bei dem zugleich ein es am Satzanfang steht. Die Verbform steht in der 3.Ps.Sg. Ein wichtiges Merkmal des unpersönlichen Passivs ist, dass das es nur an erster Stelle möglich ist. Wird die Anfangsposition durch ein anderes Vorfeldelement gefüllt, so verschwindet es. In diesen Fällen bleibt nur die Verbform in 3.Ps.Sg. übrig. M.E. ist es hier möglich, eine spezifische, rein morphemische Variante der [es+3.Ps.Sg.]-Konstruktion ohne es anzunehmen:

\footnotetext{
${ }^{104}$ Die Notation lässt (also die 3.Ps.Sg.-Form) ist wichtig, weil sie zeigt, dass hier nur diese Form möglich ist (nicht etwa lassen), und so bleibt die Wirkung der [es+3.Ps.Sg.]-Konstruktion nicht verdeckt. Das tiefgestellte „trans“ bedeutet, dass es hier auch um De-Transitivierung geht.
} 


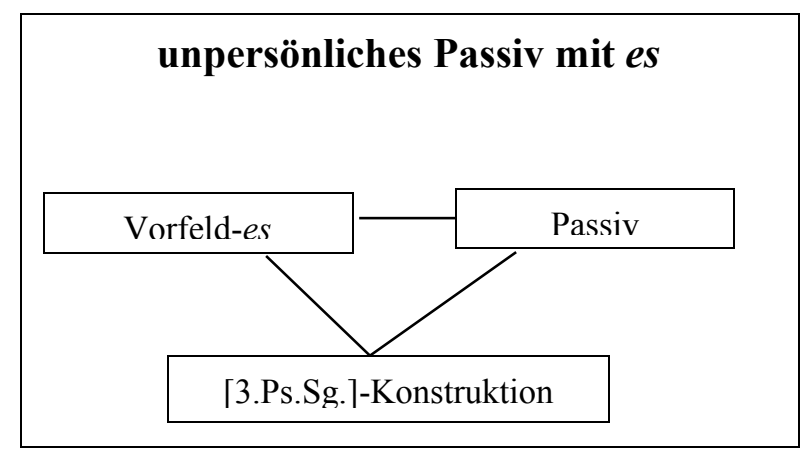

Das es, das hier am Satzanfang erscheint, ist nicht das der [es+3.Ps.Sg.]-Konstruktion. Es ist ein Vorfeld-es, das immer verschwindet, wenn das Vorfeld durch ein anderes Element besetzt wird. In diesem Fall bleiben nur noch das Passiv und die morphemische [3.Ps.Sg.]Konstruktion. Unpersönliches Passiv entsteht also, wenn das Passiv und die [3.Ps.Sg.]Konstruktion gleichzeitig Anwendung finden. Das Vorhandensein eines Vorfeld-es ist im Grunde genommen unabhängig davon, da es in persönlichen Kontexten genau so gut stehen und das eventuell vorhandene Subjekt rhematisieren kann, vgl::

(152) Die Sprache des Epos spricht mich an; es liegt der kindlich naive, kräftig deutsche Ton darin (101) (SCH)

Auch Welke (2002: 300) hebt die Beziehung zwischen [es+3.Ps.Sg.] und unpersönlichem Passiv - in Anlehnung an Dal (1966) - hervor und macht darauf aufmerksam, dass in historischer Sicht eine vom „normalen“ Passiv teilweise selbstständige Entwicklung des unpersönlichen Passivs vorstellbar ist, nämlich im Anschluss an impersonale esKonstruktionen, die als Muster dienen. Somit ist das Auftreten von es im unpersönlichen Passiv nicht nur dadurch erklärbar, dass eine Subjekt-Prädikat-Struktur hergestellt werden soll, sondern es handele sich dabei um eine Analogie zu impersonalen Konstruktionen mit sein/werden und es. Gegen diese These spricht jedoch, dass das es im unpersönlichen Passiv - falls vorhanden - m.E. ein Vorfeld-es ist, das bei anderer Vorfeldbesetzung verschwindet, was für impersonale es-Verwendungen sonst nicht zutrifft.

Es stellt sich an dieser Stelle die Frage, was der Unterschied zwischen Passiv und der [es+3.Ps.Sg.]-Konstruktion ist, wenn beide zur Herstellung der Geschehensperspektive (Welke 2002) dienen und zu Agens-Dezentrierung führen (Ágel 1997: 154f.). Ich glaube, die [es+3.Ps.Sg.]-Konstruktion kodiert grundsätzlich Unpersönlichkeit, das Passiv setzt aber ein handlungsfähiges Agens voraus, das dann in den Hintergrund tritt. Diese Differenz 
drückt Goldberg (1995: 57ff.) mit den beiden Termini „shading“ (hier: für das Passiv) und „cutting“ (hier: für die [es+3.Ps.Sg.]-Konstruktion) meiner Meinung nach treffend aus. Beim Passiv wird die Rolle des Agens nur ,verdeckt“ (shading), bei der [es+3.Ps.Sg.]Konstruktion findet ein radikalerer „Eingriff“ statt (cutting) oder es wird überhaupt kein intentional Handelnder angenommen (Typ es regnet).

Zum Schluss soll noch anhand der Beispiele (143) bis (145) auf den Begriff der „,inheritance“ (,Vererbung ${ }^{e}$ ) eingegangen werden, dem in der Konstruktionsgrammatik eine wichtige Rolle zukommt. Dabei geht es darum, dass bestimmte Konstruktionen miteinander analogisch verwandt sind und aufeinander bezogen werden können, wobei eine Grundkonstruktion als Ausgangspunkt für andere anzunehmen ist, vgl. „central and related senses“ in Goldberg (1995: 37f.) und Kálmán (2001: 30ff.). Genau das ist auch in der Radical Construction Grammar Crofts (2002: 17) mit „schematic“ und „specific“ constructions gemeint. Dies betrifft die oben dargestellte Verbindung der Witterungsimpersonalia mit okkasionellen Ereignisverben oder mit Konstruktionen, in denen ein Kopulaverb steht (s. (145)). Dabei zieht die Frage nach den Vererbungsverhältnissen auch die nach der sprachhistorischen Motivierung dieser nach sich. Bezüglich des Verhältnisses zwischen Witterungsimpersonalia und anderen, analogen Konstruktionen könnte man eine zentrale Konstruktion postulieren, die Beispiel (143) repräsentiert und zu der (144) und (145) analoge Varianten darstellen. Auch (146) stellt eine [es+3.Ps.Sg.]-Konstruktion dar, es kann jedoch nicht direkt aus Witterungsimpersonalia abgeleitet werden. Trotzdem kann man sagen, dass alle Beispiele in (143)-(145) ein „Denkmodell“ im Bühler'schen Sinne darstellen, nämlich das der Impersonalia, die Geschehnisse, Vorgänge oder Zustände ausdrücken. Croft (2002: 28) spricht hier von einem „conceptual space“. Schematisch sieht dies wie folgt aus:

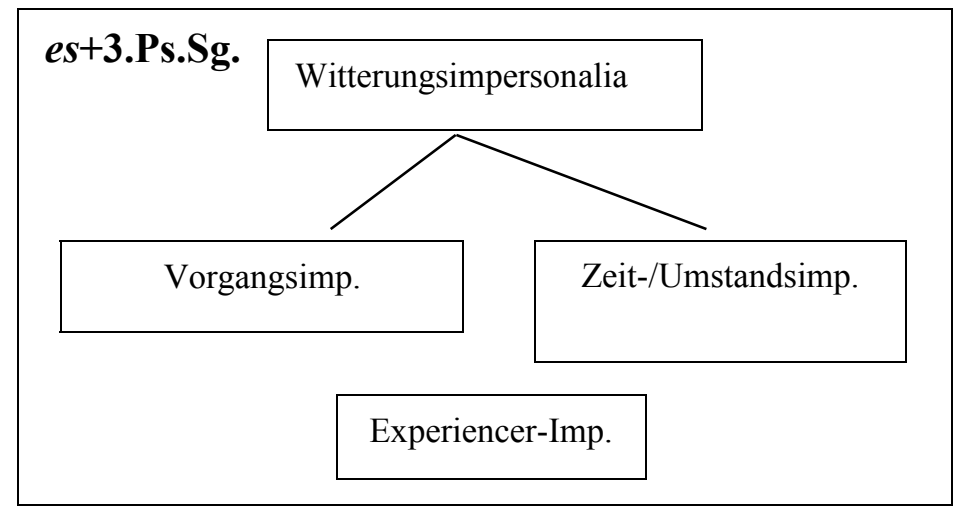


„Witterungsimpersonalia“ steht für Fälle wie die in (143), „Vorgangsimpersonalia“ für Fälle wie die in (144), „Zeit-/Umstandsimpersonalia“ für Fälle wie die in (145). Jedes Kästchen steht für eine spezifische Konstruktion, alle sind bezüglich der Konstellation [es+3.Ps.Sg.] fest. Dass (146) (Experiencer-Imp.) mit den anderen nicht verbunden ist, weist darauf hin, dass es - trotz des [es+3.Ps.Sg.]-Status - eine andere sprachhistorische Entwicklung aufweist (vgl. Ágel 2000a: 1872). Ebenfalls die sprachgeschichtlichen Zusammenhänge betrifft die Frage, wie Analogie bzw. die analogische Beziehung der zu Grunde liegenden Konstruktion auf andere Strukturen (s. okkasionelle Ereignisverben) mit einer stufenweisen Entwicklung im grammatikalisierungstheoretischen Sinne verbunden werden kann, vgl. dazu Traugott (2008: 22 und 23ff.) Eine mögliche Antwort könnte die Auffassung Himmelmanns (1997: 31) bieten, der den Begriff Grammatikalisierung nicht nur auf Wort-, sondern auch auf syntaktischer (Ausdrucks-) Ebene interpretiert ${ }^{105}$ :

In Grammatikalisierungsprozessen wird nicht nur ein Element [hier: das es, D.C.], das Grammem, sondern ein Ausdrucksmuster (eine Konstruktion) grammatikalisiert.

Grammatikalisierung manifestiert sich nach dieser Ansicht sowohl auf der Ebene des jeweiligen Elements als auch auf der Ebene der Konstruktion, in der es vorkommt; diese beiden Ebenen korrelieren also miteinander. Für beide Ebenen besteht Grammatikalisierung in der „Ausweitung des Gebrauchskontextes“ (ebd., 28), wobei unter Gebrauchskontext sowohl der semantisch-pragmatische wie auch der syntaktische Kontext zu verstehen ist. Im Falle von es könnte man dieser Ansicht folgend davon ausgehen, dass wir es nicht nur mit der Grammatikalisierung des Elementes es an sich zu tun haben, sondern dass sich Grammatikalisierung in bestimmten grammatisch-semantischen Kontexten vollzieht, in denen es vorkommt. In Anlehnung an Himmelmann (1997) beinhaltet die Grammatikalisierung (Ausweitung des Gebrauchskontextes) des Wortes es folgende Parameter:

Koaleszenz: Tendenz zur Klitisierung (gibt's, regnet's, geht's)

Unbetontheit: schwache Pro-Form (im Vergleich zu das z.B.)

Generalisierung der Phorik: Bezug nicht nur auf NPs (s. abstrakte Objekte)

\footnotetext{
${ }^{105}$ In Himmelmanns Arbeit (1997) geht es um die Grammatikalisierung nominaler Ausdrücke.
} 
Abbau syntaktischer Restriktionen: Aufgabe von Genus-/Numeruskorrespondenz

Diese Veränderungen gehen mit Veränderungen der syntaktischen Struktur einher. Im Falle von [es+3.Ps.Sg.]-Konstruktionen geht es darum, dass zusammen mit es die Einheitenkategorie 3.Ps.Sg. zu einer unpersönlichen Kategorie grammatikalisiert wird. Sie ist nicht mehr die Mikroform für Pro-Formen (wie er/sie/es in ihrer persönlichen Variante), sondern stellt eine in der Konstruktion grammatikalisierte Mikroform dar. Dies kann man sowohl an einem Beispiel für ,substantive/lexically filled“ Konstruktionen (Fillmore/Kay/O 'Connor 1988: 505) wie es gibt als auch an den okkasionellen Ereignisverben veranschaulichen. Das es bei es gibt lässt sich auf ein ursprünglich phorisches es zurückführen, das als reine Anapher sich auf den sprachlichen Kontext bezog (Scheibl 2000: 376), wobei geben in der Bedeutung, erzeugen \%,hervorbringen ${ }^{\circ}$ verwendet wurde (s. 3.1). In es gibt finden wir dann das grammatikalisierte es und ein sekundär paradigmatisiertes geben in obligatorischer 3.Ps.Sg.-Form. Wichtig ist, dass sich dieser Prozess in der Konstruktion es+gibt abspielt, in der auch die Grammatikalisierung von geben stattfindet (Lenz 2007). Was okkasionelle Ereignisverben anbelangt, so ließe sich eine stufenweise Grammatikalisierung des es und der 3.Ps.Sg.-Form in den Kontexten annehmen, in denen herkömmlich persönlich gebrauchte Verben stehen. Eine genaue Rekonstruierung dieses Phänomens wird allerdings erst möglich, wenn das alt-, mittel-, frühneuhoch- und neuhochdeutsche Belegmaterial von Näß1 (1996) auf solche Kontexte hin untersucht wird. Möglich wäre dann eventuell die Erschließung so genannter kritischer und isolierender Kontexte (Diewald 2006: 4f.). Der kritische Kontext

is characterized by multiple structural and semantic opacity, thus inviting several alternative interpretations, among them the new grammatical meaning. The critical context functions as a kind of catalyst $[\ldots]$

Der isolierende Kontext

shows the consolidation of the grammaticalization process, i.e. the reorganization and differentiation of the grammatical formatives and the paradigm that is the target category of the ongoing grammaticalization process. In this phase, the new grammatical meaning is isolated as a separate meaning from the older, more lexical, meaning. This separation of the two meanings is achieved by the development of isolating contexts for 
both the lexical and the grammaticalized readings, i.e. specific linguistic contexts that favour one reading to the exclusion of the other. (ebd., 5)

Eine Untersuchung der Belege von Näßl und der in meinem Korpus unter dem Gesichtspunkt kritischer und isolierender Kontexte bzw. der Inbeziehungsetzung kritischer mit isolierenden Kontexten kann in der vorliegenden Arbeit nicht erfolgen. Es sei nur kurz darauf hingewiesen, dass Diewald (2006) solche Kontexte als Konstruktionen ansieht, was die Einbindung solcher Überlegungen in die in diesem Kapitel ermöglichen könnte. Eine solche Herangehensweise bzw. der enge Konnex von Grammatikalisierungsforschung und Konstruktionsgrammatik verspricht auch neue Einsichten in bekannte Themen, vgl. die einschlägigen Gedanken Knoblochs (2009: 388):

Hier fasziniert KG, weil sie begreifbar macht, wie bei der Grammatikalisierung von lexikalischen Elementen in konstruktionalen Kontexten lexikalische Autonomie ab- und konstruktionale Autonomie aufgebaut wird, wie aus einer ,untypischen ${ }^{\circ}$ und hoch implikativen Verwendung schrittweise ein expansives ,modellbildendes ${ }^{e}$ Muster entsteht, welches das grammatikalisierte Element bindet und gegenüber seinen ,freien lexikalischen Bedeutungen isoliert.

\subsection{Empirie: Fallanalysen}

In diesem Kapitel sollen Belege präsentiert werden, die die Überlegungen zu Konstruktionen und Valenzsimulationen in 3.3 ergänzen. Es werden dabei zwei Themenbereiche behandelt:

a) okkasionelle Ereignisverben (Näß1 1996) und „substantive/lexically filled“ Konstruktionen (Fillmore/Kay/O'Connor 1988: 505)

b) [es ist so]-Konstruktionen 
ad a)

Bei den okkasionellen Ereignisverben sind in erster Linie Belege interessant, in denen regulär PPs vorkommen:

(153) so müßen den viele armen schafflein vor hunger und durst verschmachten und umbkommen, wegen solchern untreuen hirtten. so bleibt es doch bë̈ den wortten des propheten, Ich wil einen hunger p. O! (47) (NE)

(154) Zur 3. Wurzelziehen ist es heute nicht mehr gekommen. (636) (LB)

(155) Morgen Abends erwarte ich Anna, Du siehst an Abwechslung fehlt's mir nicht... (787) (LB)

(156) Jetzt ist's halt wieder aus mit der Freiheit... (1689) (LB)

(157) Als die Zimmerleuth' fertig waren, giengs ans Mauern. (13) (B)

(158) Diese Krankheit kam mir so ekelhaft vor, daß ich's sogar bey meinen Kindern nie recht ohne Grausen aushalten konnte. (22) (B)

(159) Wie ist's mit Deiner Reise, ich weiß ja diesmal gar nicht wohin Du gehst. (513) (LB)

(160) Mir ist es halt nicht gegönnt, Dir dies Ringl so meuchlings an die Hand zu stecken, wie Du's mir gethan, denk' aber indem Du es an Deinem Finger siehst, daß ich's im Geiste thue und daß ich Dich bitte es zu tragen zum Zeichen unserer Herzensgemeinschaft und daß endlos wie der Ring auch meine Liebe zu Dir ist. Schreibe mir halt ob ich's mit der Weite getroffen habe, sonst müßte er gerichtet werden. (73) (LB)

Aus diesen Beispielen ist ersichtlich, dass das es sowohl als simuliertes Subjekt als auch als simuliertes Objekt (s. (158) und (160)) fungieren kann. Die PPs sind in (153) bis (156) obligatorisch und ihre Form ist insofern konstant, als jeweils andere Präpositionen nicht gewählt werden können. Dabei sind die PPs direkt an die Verbindung aus es + Verb 
gebunden, was zur Folge hat, dass sie als Präpositionalobjekte anzusehen sind. (157) und (158) sind hinsichtlich der PP etwas anders zu analysieren: In diesen Belegen ist nicht die PP an sich, sondern nur das dadurch kodierte Direktivum (s. (157)) bzw. das Lokaladverbial (s. (158)) notwendig. In (159) und (160) schließlich finden wir mit-PPs, die das Thema angeben, von dem die Rede ist.

Bei Belegen des obigen Typs bietet sich m.E. ein Vergleich mit anderen ähnlichen Beispielen an:

(161) Der hiesigen Großmutter geht es schlecht. (23) (K)

(162) Naja, es gab einen offiziellen Sprachton, den sprachen alle Verantwortungsträger (3) (SB)

(163) ich bitt Dich denn sieh, es handelt sich ja wahrhaftig nicht drum, daß ich als Richter über Dir sitzen will (16) (L)

Auch in (161) bis (163) werden bestimmte Ausdrucksformen regiert: in (161) der Dativ, in (162) der Akkusativ und in (163) die PP mit um und das Reflexivum sich. In all diesen Fällen sind die Rekta an die Verbindung es + Verb gebunden. Trotzdem scheint es einen wesentlichen Unterschied zu geben zwischen diesen Belegen und den Beispielen (153) bis (160). Dieser Unterschied besteht in der Semantik des Verbs bzw. darin, dass die Bedeutung der Verben in (153) bis (160) sich im Wesentlichen nicht ändert, während in (161) bis (163) eine völlig neue Bedeutung vorliegt, die mit der ursprünglichen ohne es wenig zu tun hat. Im ersten Fall findet also „nur“ eine Umperspektivierung statt, indem die zum Verb gehörenden semantischen Rollen anders kodiert werden. Am Beispiel von gehen lassen sich die oben beschriebenen Verhältnisse veranschaulichen: In der persönlichen Verwendung sieht gehen ähnlich wie andere agentive Verben mit herkömmlichem Subjekt aus. In einem zweiten Schritt ist es möglich, gehen gelegentlich als unpersönliches Verb zu gebrauchen, indem die in 3.3.2 dargestellte [es+3.Ps.Sg.]-Konstruktion Anwendung findet, was dazu führt, dass das es als unpersönliches, formales Subjekt interpretiert wird, vgl. (157). Der Akzent liegt dabei auf dem Umstand, dass das Gehen in diesem Fall wie ein Ereignis oder Vorgang perspektiviert wird. Schließlich finden wir in (161) gehen in einer anderen Bedeutung als der ursprünglichen vor. Dabei gilt jedoch auch hier, dass zu dieser Bedeutung 
ebenfalls ein es und die drittpersonige Form des Verbs nötig sind. Die Differenz zwischen es geht $X$-wohin und es geht jm X-wie kann man unter Rückgriff auf die Unterscheidung formaler grammatischer Konstruktionen von „lexically filled“ Konstruktionen bei Fillmore/Kay/O'Connor (1988: 505) gut fassen. Okkasionell unpersönlich gebrauchte Verben sind in diesem Sinne formale/grammatische [es+3.Ps.Sg.]-Konstruktionen (vgl. hierzu auch 3.3.2), während die lexikalisierten Formen als „lexikalisch gefüllt“ betrachtet werden können.

Interessant sind des Weiteren Belege, die zeigen, dass die Grenze zwischen phorisher und nicht-phorischer Verwendung von es nicht immer klar gezogen werden kann:

(164) Jch wußte nicht, mit wem ich's zu thun hatte. (13) (MD)

(165) Nach diesen mich unangenehm berührenden Mittheilugen gingen wir zu Marie Schwarz zum Kaffee; Milia fand es recht behaglich dort (107) (SCH)

(166) Bachmann hingegen hatte noch von seinem Handgeld übrig, gieng in die Schenke, und ließ sich's wohl schmecken; nur etwa zu vornehmen Häusern, Pfarrhöfen und Klöstern, kam er auch mit. (5) (B)

Während ein phorischer Bezug auf einen Vorgängerausdruck in (164) nicht in Frage kommt, halte ich es für möglich, in (165) und (166) Phorik anzunehmen. (165) könnte als ein Beispiel für Bezüge auf Situationen, (166) als eines für schemabasierte implizite Phorik, wie sie in 2.2.1 dargelegt wurde, gedeutet werden. In (165) wäre demnach davon auszugehen, dass es auf die (angenehme) Situation beim Kaffetrinken bei Marie Bezug nimmt, während in (166) gieng in die Schenke implizit als Quelle für Essen und Trinken gilt. Das es nimmt dann auf dieses Essen und Trinken Bezug. Auch beim nominativischen es kann man ähnliche Belege finden, deren Zuordnung nicht eindeutig vorgenommen werden kann, vgl. z.B.:

(167) Ich fing an zu beten und dann weinte ich so, dass es mich schüttelte, bis ich gerufen wurde. (8) (SCHG) 
In diesem Beleg sind meiner Meinung nach zwei Interpretationen möglich: Entweder man analysiert es schüttelt jn als einen Fall von okkasionellen Ereignisverben - vgl. dazu auch Beleg (135) in 3.1 - oder aber man nimmt wortsemantisch basierte implizite Phorik an, wie sie im Falle von Wortbildungen in 2.2.1 geschildert wurde, vgl. hierzu auch Beleg (25) dort. In (167) könnte demnach Weinen als Implikat zu weinte ich so angegeben werden.

Die so genannte [es ist so]-Konstruktion wird in Günthner (2009) detailliert beschrieben. Zur Veranschaulichung sei an dieser Stelle folgender Beleg angeführt:

(168) Aber natürlich, den ganz negativen Anstrich an diesem Dialekt haben die Kommunisten gegeben, weil eben Sachsen dummerweise die meisten dieser Kommunisten gestellt hat, und die Kommunisten ja alle sächsisch sprachen, weil sie aus dieser Gegend hier kamen...vielfach zumindestens....Das hat natürlich dann „Kommunismus gleich sächsische Sprache“ eingebracht. Das ist nun einmal so ... Es ist einfach so...daß die „rote“ kommunistische Arbeiterbewegung hier in Sachsen doch am allerausgeprägtesten war, und daß die roten, kommunistischen Funktionäre natürlich aus Sachsen kam, weil die Partei hier am stärksten war. (18) (SB)

Günthner (2009) behandelt KVen als „Extrapositionskonstruktionen“ und untersucht beide Teile der KV (d.h. Hauptsatz mit Korrelat und Nebensatz) hinsichtlich Semantik und Form. Dabei stellt sie (2009: 37) das „syntaktische Muster“ [es ist so] neben klassische KorrelatFälle. Für diese Parallele spricht unter semantischem Gesichtspunkt, dass auch durch das Muster [es ist so] Komplemente eingeführt werden. Unter syntaktischem Aspekt wiederum ist zu sehen, dass bezüglich der Form der korrelierten Nebensätze, genau so wie bei herkömmlichen KVen, Varianz vorliegt (ebd., 38), vgl. bspw.

(169) Es war also durchaus nicht so, daß sich da säuberlich getrennte Gruppen gegenüberstanden. Es war mehr so ... als ich dort in Z. war, das war ja eine verhältnismäßig späte Zeit (5) (SB)

In (169) gibt es zwei Belege für [es ist so]. Im ersten finden wir einen dass-Satz mit Verbletztstellung, während im zweiten erst ein Adverbialsatz eingeschoben und dann die [es 
ist so]-Konstruktion fortgesetzt wird, wobei der zweite Teil eine Hauptsatzstruktur mit Verbzweitstellung aufweist. Andererseits gibt es auch Differenzen zwischen [es ist so] und herkömmlichen KVen. So sei der [es ist so]-Teil der KV „semantisch (annähernd) leer“ (ebd., 40). Außerdem gilt:

Während der A-Teil (es ist so) gerade aufgrund seiner semantischen Entleerung eine nahezu ausschließlich pragmatische, diskursfunktionale Rolle innehat, enthält der BTeil die zentrale Information. es ist so-Konstruktionen stehen demnach als Ressource zur Verfügung, um eine monoklausale Aussage (wie ,in den meisten Fällen ist das eigentlich nicht notwendig") in eine komplexere Form zu packen, die die kognitivpragmatische Funktion hat, dem Inhalt der eigentlichen Aussage (dem B-Teil) mehr Gewicht zu verleihen und die Aufmerksamkeit des Gegenüber darauf zu lenken (ebd., 41).

Die Analysen Günthners sollen in zwei Punkten ergänzt werden. Einerseits soll die Annahme der „semantischen Leere“ kommentiert werden. Im Anschluss daran soll auf die Varianz im [es ist so]-Teil eingegangen werden.

Was die „semantische Leere“ angeht, so würde ich ausgehend von (168) dafür argumentieren, dass das es in dieser [es ist so]-Konstruktion nicht leer ist. Möglich ist m.E. die Annahme summierender Anaphorik auf den Vorgängerkontext. Das es verweist demnach auf das Vorangehende als Ganzes (vgl. „sum of events“ in Asher 1993: 235 und die Überlegungen in 2.2.2). Mit Admoni (1976: 220) lässt sich daher sagen: „Allerdings ist oft das, was durch es vorweggenommen ist, doch auf irgendwelche Weise in vorhergehendem Text semantisch vorbereitet.“ Für diese Sicht spricht m.E. auch folgender Beleg:

(170) Konnte sie aber nicht im Wolmeinen gegen Dich, allerdings ohne Deinen Auftrag, dann aber nur um so mehr im Wolmeinen mit Dir der andern bemerkl machen daß sie dich unmutig gemacht? Und ich glaub auch da, daß es so ist (15)

Aus (170) ist ersichtlich, dass das Muster [es ist so] auch nachgestellt möglich ist, wobei das es anaphorisch summierend ist.

Ergänzt wird die soeben beschriebene anaphorische Funktion des es durch eine, die es als Teil der [es ist so]-Konstruktion hat, so wie sie in Günthner (2009) dargelegt wird. 
Diese Interpretation hat zur Folge, dass das es in solchen Fällen biphorisch ist, vgl. dazu näher 2.3.3. Seine Kataphorik ist dabei, wie dies bei anderen Korrelaten auch zu sehen ist, gebunden. Dabei ist diese Gebundenheit insofern anders als bei anderen Korrelaten, als sie hier mit einer pragmatischen Funktion zusammenhängt: Günthner (2009: 29, 40, 42) spricht dabei von Rahmung und Ankündigung salienter Informationen. Der [es ist so]-Teil dient also als Rahmung für den korrelierten Folgesatz. Durch diese Art Rahmung wird die Fokussierung des Folgesatzes möglich:

Durch die vorgelagerte Produktion eines Rahmens für einen noch nicht thematisierten, aber bereits projizierten Sachverhalt wird die Aufmerksamkeit der RezipientInnen auf diese noch zu identifizierende Konstituente gelenkt, was wiederum deren Salienz erhöht. (ebd., 23).

An dieser Stelle ergibt sich meiner Ansicht nach die Möglichkeit, [es ist so]-Konstruktionen neben Spaltsätze zu stellen, die ja auch eine mögliche Strategie der Fokussierung darstellen, vgl. dazu Fischer (2009) und 2.4. So ist es durchaus sinnvoll, es ist $X$, der/die/das (Spaltsatz) und es ist so, dass als strukturell ähnliche Formate zu betrachten. In beiden Fällen geht es nämlich darum, dass einfachere Strukturen aufgespalten werden, um dabei Fokussierungen vorzunehmen, vgl.:

(168) [...] Es ist einfach so...daß die „rote“ kommunistische Arbeiterbewegung hier in Sachsen doch am allerausgeprägtesten war, und daß die roten, kommunistischen Funktionäre natürlich aus Sachsen kam, weil die Partei hier am stärksten war. (18) (SB)

statt

(168') Die „rote“ kommunistische Arbeiterbewegung war hier in Sachsen doch am allerausgeprägtesten.

bzw.

(171) Vielleicht ist es auch die Trauer, die sie so schweigsam macht (22) (K)

statt

(171') Die Trauer macht sie so schweigsam. 
Was die Varianz von [es ist so] anbelangt, so sind in meinem Korpus Belege anzutreffen, die über die Erweiterung und Modifzierung von [es ist so] um Adverbialbestimmungen und andere Einheiten, wie dies in Günthner (2009: 41) zu sehen ist, hinausgehen, vgl.:

(172) Denn es ist hir in ähnlicher Weise wie bei den Bekenntnißschriften, daß man nicht das gegebene erforschen will, sondern selbst erst etwas aufrichten. (9) (L)

(173) denn die jetzige traurige Zeit hat den fröhlichen Übermut von allen Menschenkindern abgestreift, es ist wie wenn über allen Menschen ein Trauerschleier niedergefallen wäre $(22)(\mathrm{K})$

(174) er wüste nur nicht | wo sein schwager bliebe, oder wie es seij $\mid$ das er nicht komme (8) (DM)

Die Varianz ist in (172) bis (174) ist insofern stärker als in Günthners Beispielen (2009: 41), als das so fehlt bzw. durch andere Elemente ersetzt wird. In (172) finden wir den semantisch sehr ähnlichen Ausdruck in ähnlicher Weise, in (174) wird der Rahmungsteil selbst von wüste regiert und fungiert als satzförmiges direktes Objekt, wobei statt so das $w$-Element wie steht. Weiterhin ist auf die Konjunktivform der Kopula hinzuweisen. Trotz dieser Abweichungen ist meiner Meinung nach auch (174) funktional so zu deuten wie [es ist so]. Etwas anders sind die Verhältnisse in (173), denn hier hat man es mit einem vergleichenden Relativsatz zu tun, der durch den Subjunktor wie wenn eingeleitet wird. Dies kann jedoch als eine semantische Modifizierung, die die Inhaltsrelation zwischen es ist und dem wiewenn-Teil betrifft, betrachtet werden. 


\section{Zusammenfassung}

In diesem abschließenden Kapitel sollen die Ergebnisse der vorliegenden Arbeit zusammenfassend dargestellt werden. Im Mittelpunkt stehen dabei die Typenbildung und Überlegungen zum es-Gesamtsystem. Ergänzt werden soll das Bild durch Hinweise auf Forschungsfragen, die in der vorliegenden Arbeit nicht besprochen werden konnten, die aber relevant sind und die Möglichkeit der Einbettung der Ergebnisse der vorliegenden Arbeit in einen breiteren Forschungs- und Diskussionszusammenhang zeigen. Der Diskussion der Ergebnisse soll an dieser Stelle ein kurzer Rückblick auf das in 1.2 formulierte Anliegen der Arbeit vorausgehen.

In der vorliegenden Arbeit wurden zwei Hauptziele verfolgt. Diese doppelte Zielsetzung wurde funktional gerechtfertigt, indem zwei Haupttypen von es angenommen wurden: das phorische und das nicht-phorische es:

(1) Beim phorischen es stand zunächst im Fokus, wie Phorik an sich und im Verhältnis $\mathrm{zu}$ anderen verwandten Termini zu interpretieren ist. In einem zweiten Schritt wurde davon ausgegangen, dass sich eine Trennung der semantischen Ausbuchstabierung der Phorik von der morphosyntaktischen als sinnvoll erweist. Dieser Methodik entsprechend wurde dann ein System der es-Phorik entwickelt, indem zuerst auf die Semantik und dann auf die Morphosyntax eingegangen wurde. An dieses Vorgehen wurde auch die Annahme der drei Phorikarten implizit, explizit und gebunden gekoppelt. Neben allgemeinen Überlegungen zur Typenbildung wurden auch Beispieltypen (z.B. nicht-korrespondierende NP-Bezüge) analysiert und erläutert, denen in der Forschungsliteratur wenig Aufmerksamkeit geschenkt wurde.

(2) Beim nicht-phorischen es wurde der Akzent auf seinen grammatiktheoretischen Status gelegt. Unter Rückgriff auf neuere grammatiktheoretische Ergebnisse wurde der Versuch unternommen, nicht-phorische es-Vorkommen valenztheoretisch und im Rahmen der Konstruktionsgrammatik zu analysieren. Dabei wurden zunächst die einschlägigen Theoriebausteine erörtert. Im Anschluss daran wurden die entsprechenden Festlegungen am empirischen Material veranschaulicht. 
Im Hintergrund der oben skizzierten doppelten Zielsetzung stand in Anlehnung an Admoni (1976) das Bestreben nach einer Darstellung, die auch Zusammenhänge zwischen einzelnen es-Typen sichtbar macht. Auch auf diesen Punkt soll im Folgenden eingegangen werden.

In einem ersten Schritt soll die Typenbildung im phorischen Bereich zusammenfassend präsentiert werden. Zweitens soll es darum gehen, wie nicht-phorische es-Typen dargestellt werden können. Abschließend soll der Versuch unternommen werden, die beiden Haupttypen engzuführen, soweit dies unter Rückgriff auf die Begrifflichkeit und die Festlegungen in der vorliegenden Arbeit möglich ist.

Vor dem Hintergrund der Feststellung, dass Phorik durch es sowohl unter semantischem wie auch syntaktischem Aspekt beschrieben werden kann, wurde in Kapitel 2 die Forderung nach einer getrennten Behandlung von Semantik und Syntax bei der esPhorik formuliert. Einem solchen Vorgehen liegt die Beobachtung zugrunde, dass bei einem Satz wie etwa Ich habe ein Buch gekauft, es war sehr teuer. bezüglich der Verweisleistung von es zwei Aussagen möglich sind: Entweder man weist auf die Bezugnahme auf eine Nominalphrase durch es hin oder aber man betont, dass hier auf einen Gegenstand Bezug genommen wird. Beide Redeweisen sind legitim und in der vorliegenden Arbeit wurden sie beide entsprechend ausgeführt. Relevant wird diese Methodik vor allem, wenn typische Konstellationen zwischen Semantik und Syntax herausgestellt werden. Bevor dies an dieser Stelle zusammenfassend geschieht, soll eine kurze Darstellung sowohl der semantischen wie auch der syntaktischen Seite in komprimierter Form erfolgen. Unter semantischem Gesichtspunkt wurden unter Berücksichtigung entsprechender Belege zunächst implizite und explizite Verweise unterschieden, vgl. hierzu Kapitel 2.2.1. Bei impliziten Verweisen ging es im Wesentlichen darum, dass Elemente im Vorgängerkontext Bezugsobjekte implizieren, auf die durch es verwiesen wird. Dabei wurde in Anlehnung an die Forschungsliteratur zu indirekten Anaphern (s. u.a. Schwarz 2000) zwischen wortsemantisch basierten impliziten Verweisen und solchen unterschieden, bei denen die Bezugnahme durch es auf Implikate zurückgeführt werden kann, denen nicht einzelne Wörter, sondern Schemata zugrunde liegen. Im Anschluss an die Darstellung impliziter Bezugnahme wurden explizite Verweise untersucht, bei denen das Bezugsobjekt nicht impliziert wird, sondern im vorangehenden Textteil aufzufinden ist. Hinsichtlich der Semantik solcher Bezüge wurden verschiedene mögliche Bezugsobjekte diskutiert, wobei eine grundlegende Differenzierung zwischen Gegenständen und abstrakten Objekten 
vorgenommen wurde. Zusammenfassend lassen sich die Festlegungen in 2.2.1 und 2.2.2 wie folgt darstellen:

\section{implizite Phorik}

worsemantisch basiert

schemabasiert

\section{explizite Phorik}

Bezug auf abstrakte Objekte

- Bezug auf Situationen

- Summierender Bezug

- Bezug auf Propositionen inkl. Bezug auf das Prädikat oder seine Teile

- Bezug auf Eigenschaften

\section{Bezug auf Gegenstände}

In der Syntax (s. Kapitel 2.2.3) wurden in erster Linie Phrasen und Elementarsätze voneinander getrennt. Dabei wurde der Frage nachgegangen, was als Elementarsatz gilt und welche Phrasentypen als Ausdruckskategorien bei der Phorik eine wichtige Rolle spielen. Es entstand dabei folgendes Bild:

Bezugsgröße kategorial nicht erfassbar

Bezug auf Elementarsätze

Bezug auf Phrasen

- Bezug auf VPs

- Bezug auf NPs

- Bezug auf ADJPs 
Im Falle der Elementarsätze wurde der kataphorische Verweis auf Nebensätze gesondert diskutiert (Korrelat, s. 2.3). Es wurde dafür argumentiert, dass Korrelatverbindungen eine syntaktisch gebundene Form propositionalen Bezuges darstellen. Die besonderen Merkmale dieser Art von Verweisen haben dazu geführt, sie (neben impliziter und expliziter Phorik) als eine dritte Phorikart auszuweisen. Im Mittelpunkt von 2.3 standen dabei vor allem Überlegungen zur Integration korrelierter Nebensätze in den Hauptsatz. Es wurden anhand einschlägiger Parameter (Position des Nebensatzes, Stellung des Korrelates und Form des Nebensatzes) verschiedene Stufen der Integration vorgestellt, vgl. 2.3.3. Ergänzt wurde die Darstellung durch wenn- und Spaltsätze (vgl. dazu 2.4) als besonders stark integrierte Strukturen mit Korrelaten. An dieser Stelle soll darauf hingewiesen werden, dass die Integriertheit korrelierter Einheiten unterschiedlich bewertet werden kann, u.a. weil auch andere Parameter als einschlägig angesehen werden können, s. bspw. die Zusammenfassung hierzu in der IDS-Grammatik (1997: 2250). Außerdem gehen Pütz (1975: 23) und Zitterbart (2002a: 84) auf korrelathaltige Hauptsätze mit Kopula und Adjektiv ein (Typ es ist schön, dass [...]). Interessant sind solche Beispiele vor allem, weil behauptet wird, dass das es in diesen Fällen obligatorisch ist, wobei diese Obligatorik an die spezifische Konstellation Kopula + Adjektiv gebunden zu sein scheint (ebd.). Ausgehend von diesen Annahmen könnte, im Sinne eines Parameters, untersucht werden, welche Konstellationen bezüglich der Setzung des Korrelat-es verfestigt werden. Dies wäre nicht beispiellos, man denke nur an die auch in der vorliegenden Arbeit thematisierten lexikalisierten Korrelate wie es gilt und es heißt, vgl. 2.3.3 und 3.2. Der Unterschied zwischen diesen obligatorischen Korrelaten und den Hauptsätzen mit Kopula, Adjektiv und es besteht dann darin, dass die Obligatorik des Korrelates bei Letzteren nicht mit Änderungen in der Semantik des Verbs einhergeht. Ebenfalls als Parameter käme das Vorhandensein eines das statt eines es als Korrelat in Frage (vgl. Hegedűs 2007: 259f.). Diese Problematik ist weiterhin eng mit der grundsätzlichen Differenz zwischen es und das verbunden und betrifft somit die Gegenüberstellung von Phorik (es) und Deixis (das), s. hierzu die Überlegungen in 2.1 sowie die Belege (5) bis (7) dort. Bezüglich der Beurteilung der Integriertheit korrelierter Nebensätze wäre in diesem Sinne von Bedeutung, wenn das als Korrelat auftritt, da es durch seine deiktische Kraft den rein syntaktischen Rahmen des kataphorischen Bezuges durchbricht. Des Weiteren kommt dabei auch Biphorizität in Frage, vgl. 2.3.3 sowie Hegedüs (ebd.). 
Erwähnenswert ist im Zusammenhang mit Korrelaten bzw. deren Integration unter historischem Aspekt auch der Befund, dass in meinem Korpus unter den Belegen für propositionale Bezugnahmen eine Zunahme an gebundener Kataphorik ab ca. 1800-1850 zu beobachten ist. Während vor 1800-1850 die korrelative Subordination ca. 27\% der Belege für propositionale Phorik ausmacht, so sind es nach 1800-1850 bereits 39\%. In meinem Korpus könnte dies darauf hindeuten, dass diese integrative Technik in nähesprachlichen Texten zunimmt, wobei integrative Techniken sprachlicher Strukturierung besonders für die Distanzsprache typisch sind, vgl. dazu ausführlich Ágel/Hennig (2006). Zu einer detaillierten Analyse dieses Aspektes bedürfte es allerdings eines distanzsprachlichen Vergleichskorpus, um der Frage nachzugehen, ob in distanzsprachlichen Texten deutlich mehr Korrelate anzutreffen sind und ob distanzsprachliche Techniken auch auf die Nähesprache abfärben.

Wichtig ist bei der Darstellung der semantischen und syntaktischen Seite der es-Phorik, wie diese beiden Seiten miteinander korrelieren. Direkt korrelieren typischerweise Propositionen und Elementarsätze bzw. Phrasen und Gegenstände. Dabei ist jedoch weiter zu differenzieren, denn etwa ADJPs sind anders als NPs zu deuten. Es ergibt sich folgendes Bild:

SEMANTIK

SYNTAX

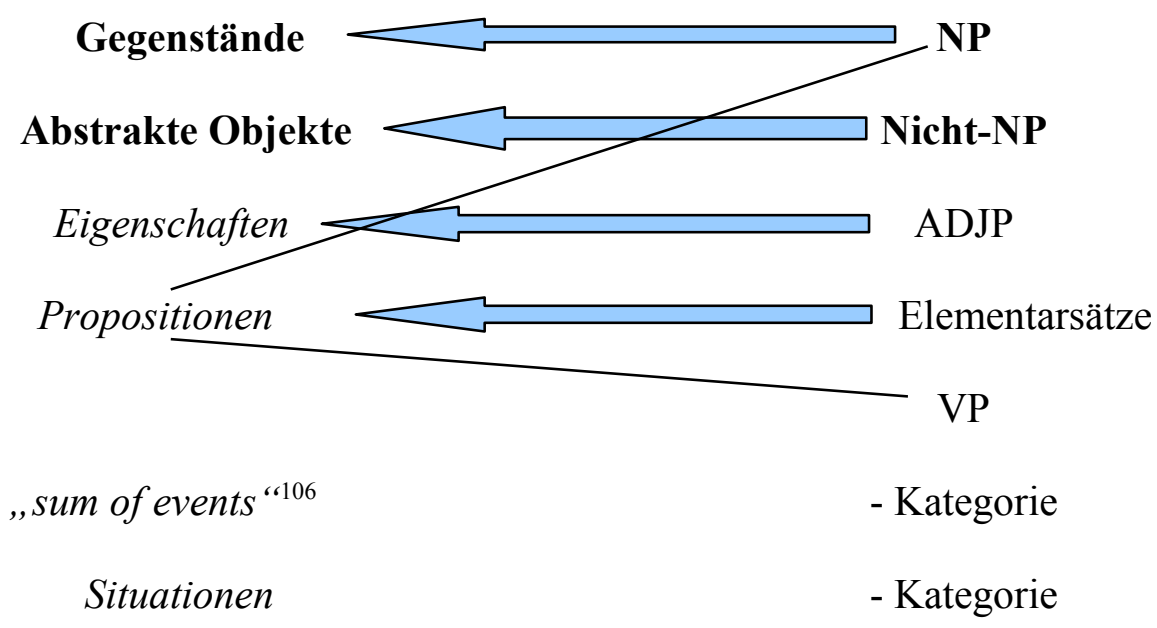

$106=$ summierender Bezug 
Die dicken Pfeile markieren typische Zuordnungen, während die einfachen Linien für weniger typische, aber dennoch anzutreffende Zuordnungen stehen. Bei summierenden Verweisen und solchen auf Situationen wurden keine Pfeile oder Linien eingetragen, weil hierfür keine direkten syntaktischen Kategorien vorhanden sind. Man könnte etwa bei summierenden Bezügen höchstens von einer Summe von Elementarsätzen ausgehen. Dabei stellt Elementarsatz keine neue Kategorie im Vergleich zu den bereits existierenden dar. Was implizite Verweise angeht, so sind prinzipiell sowohl semantisch als auch kategorial gesehen Bezüge jeglicher Art möglich, weil durch Wörter wortsemantisch basiert bzw. durch Wortverbindungen schemabasiert unterschiedliche Implikate in Frage kommen. In Askedals Beispiel (21) (1990: 21) etwa liegt ein Implikat vor, das in Form einer NP („der/die Telefonierende“) für eine Person steht (Gegenstandsnennung, vgl. Beleg (38)). Schwieriger wird es mit der Bestimmung allerdings grundsätzlich, wenn schemabasierte Phorik vorliegt (vgl. (23)), weil das implizierte Bezugsobjekt in diesem Fall auf unterschiedliche Art und Weise bestimmt werden kann, was wiederum auf die semantische und kategoriale Einordnung auswirkt. Ergänzt werden kann das obige Schema durch die Möglichkeiten der Zuordnung des es zu syntaktischen Relationen: es als Subjekt, es als direktes Objekt und es als Prädikativ. Eine es-Typisierung kann auf allen drei Ebenen, d.h. auf phorisch-semantischer, kategorial-syntaktischer und relational-syntaktischer Ebene, vorgenommen werden:

$\begin{array}{lcc}\text { Semantik } & \text { synt. Kategorie } & \text { synt. Relation } \\ \text { Pro-Gegenstands-es } & \text { Pro-NP-es } & \\ \text { Pro-es für abstr. Obj. } & \text { Pro-Nicht-NP-es } & \text { Subj./dir. Obj. } \\ \text { Pro-Eigenschafts-es } & \text { Pro-ADJP-es } & \\ \text { pro-propositionales } e s & \text { pro-sententiales } e s & \text { Prädikativ } \\ \text { summierendes } e s & - & \text { Subj./dir. Obj. } \\ \text { pro-situationales } e s & - & \text { Subj./dir. Obj. } \\ \end{array}$

Auf semantischer Ebene gibt es zwei Haupttypen (fett gesetzt), zu denen jeweils Subtypen gehören (eingerückt). Ähnlich ist es auf der kategorialen Ebene, während hinsichtlich der syntaktischen Relationen mit drei Hauptypen zu rechnen ist. Dabei ist eine Untergliederung 
des Subjekt- und des Objekt-es möglich, wenn formale Subjekte und Objekte angenommen werden. Dies betrifft aber schon den nicht-phorischen Bereich.

Nach der Diskussion phorischer es-Typen sollen nun die Ergebnisse der Analysen zum nicht-phorischen Bereich zusammengefasst werden. Bei der Analyse nicht-phorischer esVorkommen wurde der Akzent auf deren grammatiktheoretischen Status und ihre grammatische Beschreibung gelegt. Dabei wurde auf zwei Theorien bzw. auf bestimmte, für den Forschungszweck ausgewählte Bausteine zweier Theorien, der Valenztheorie und der Konstruktionsgrammatik, zurückgegriffen. Im Falle der Valenztheorie stand der Begriff Valenzsimulation im Mittelpunkt der Untersuchungen, vgl. 3.1 und 3.2. In konstruktionsgrammatischem Rahmen wurde der Versuch unternommen, den „klassischen“ Begriff von Konstruktionen (Fillmore/Kay/O'Connor 1988 und Fillmore 1989) auf nichtphorische es-Gebrauchsweisen anzuwenden. Zur theoretischen Fundierung wurden dabei auch andere wichtige konstruktionsgrammatische Arbeiten (Goldberg 1995 und Croft 2002) mit herangezogen. Mit Bezug auf die Analyse von nicht-phorischem es ist von großer Bedeutung, dass eine Arbeitsteilung zwischen Valenztheorie und Konstruktionsgrammatik angestrebt wurde, indem die Gesamtleistung von Verbindungen aus es und drittpersonigem Verb konstruktionsgrammatisch, die innere Struktur dieser Verbindungen und die „Konsequenzen“ der Konstruktionswirkung valenztheoretisch beschrieben wurden. So wurde eine impersonale [es+3.Ps.Sg.]-Konstruktion mit Vorgangsbedeutung und dezentriertem Vorgangsträger postuliert. Dabei wurde das es in dieser Konstruktion als ein Fall von Valenzsimulation bezeichnet und es wurde für die 3.Ps.Sg.-Form des jeweiligen Verbs sekundäre Paradigmatisierung angenommen (Ágel 2003). Im Anschluss an diese Festlegungen wurden dann in der Fachliteratur bekannte nicht-phorische es-Typen in diesem Rahmen analysiert. Es wurde dabei von einer grundsätzlich impersonalen Grundkonstruktion (Typ es regnet) ausgegangen, zu der Tochterkonstruktionen gebildet werden. Mit Croft (2002) wurde von einer schematischen und jeweils spezifischen Konstruktionen gesprochen. Die einzelnen spezifischen Konstruktionen unterscheiden sich in erster Linie dadurch, dass sie im Vergleich zu der Grundkonstruktion jeweils andere semantische Spezifikationen enthalten, vgl. 3.3.2. Auf der Basis der Ausführungen in 3.2 und 3.3 ließen sich zusammenfassend folgende Konstruktionen annehmen: 
- $\quad$ Rhematisierungskonstruktion mit Vorfeld-es: nur positionale Simulation der Subjektstelle

- $\quad$ Formale/grammatische Konstruktion [es+3.Ps.Sg]: Subjektsimulation

- Lexikalisch gefüllte Konstruktionen: Subjektsimulation (es gibt), Objektsimulation (es mit jm gut/schlecht meinen)

Die [es+3.Ps.Sg]-Konstruktionen sind auf der Folie der offenen Struktur es + 3.Ps.Sg. zu deuten, indem diese offene Struktur sowohl persönlich als auch unpersönlich gelesen werden kann (Ágel 2009):

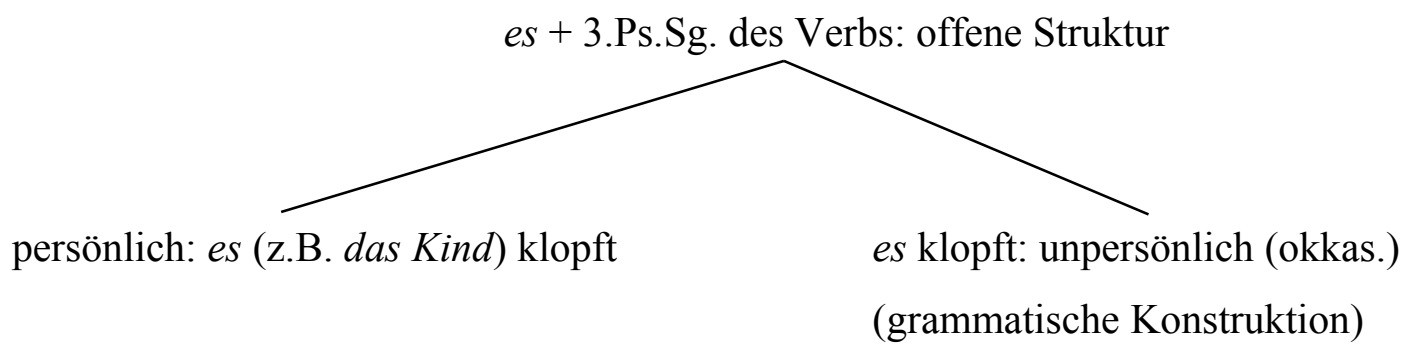

Schließlich gilt es auf Möglichkeiten der Zusammenführung des phorischen und des nichtphorischen Bereiches einzugehen:

(a) Kognitive Linguistik: Es ist möglich, es-Typen, die in der vorliegenden Arbeit nichtphorisch genannt werden, mit solchen zu verbinden, die als phorisch gelten. So setzt sich z.B. Lakoff (1987) mit englischen there-Konstruktionen auseinander und entwickelt ein Modell, in dem nicht-phorische there-Gebrauchsweisen auf die deiktische Variante von there zurückgeführt werden. Hierzu möchte ich anmerken, dass sich die Übertragung des Lakoff'schen there-Modells auf deutsche esVorkommen m.E. vor allem dann als fruchtbringend erweist, wenn sie vor dem Hintergrund derjenigen Forschungstradition erfolgt, die der Frage nachgeht, ob das impersonale es semantisch leer ist oder aber doch über eine Art Referenz verfügt. Man vergleiche hierzu etwa die (,central“ und „,noncentral“) „existentials“ in Lakoff (1987: 556, 562ff.), Harald Weinrichs „Horizontmodell“ (1993) oder Langacker 
(2007) bzw. Brugmanns Gegenargumente (1917). Zu diesem Aspekt s. auch Fußnote 16 in 2.1 .

(b) Das Korrelat: Wie in der vorliegenden Arbeit argumentiert wurde, stellt dieser Typ den Übergang vom phorischen zum nich-phorischen Bereich dar, indem die „Janusköpfigkeit“ der Korrelatfunktion erläuter wird: Das Korrelat fungiert einerseits textuell-phorisch, indem es auf satzförmige Einheiten Bezug nimmt. Andererseits ist es auch als syntaktischer Kohäsionsmarker in Korrelatverbindungen zu sehen (,struktureller Verweis“, vgl. Zifonun 2001). Ähnliches gilt für Spaltsätze des Typs es ist Peter, der [...] Fasst man nun das Korrelat nach dieser Logik als strukturelles Element, so ergibt sich die Möglichkeit, es unter dem Aspekt der Valenzsimulation zu betrachten und neben andere nicht-phorische Typen zu stellen (vgl. 3.2). M.a.W.: Die Korrelatfunktion wird „hergestellt“, indem der phorische pro-sententiale es-Typ mit der für den nicht-phorischen Bereich typischen Valenzsimulation verbunden wird.

(c) es + Kopula + NP: Ein weiteres Beispiel für die Verbindung des phorischen mit dem nicht-phorischen Bereich sind Identifizierungskonstruktionen, die - wie auch das Korrelat-es - unter verschiedenen Gesichtspunkten analysiert werden können. In einem Beispiel wie Ich sah einen Mann über die Straße laufen. Es war Peter. wird ein Verweis auf einen Mann durch es hergestellt, ein klarer Fall von (Ana)Phorik. Man beachte jedoch Folgendes:

- Fehlende Korrespondenz in Genus: Dadurch wird die Stärke der gewöhnlichen pronominalen Phorik geschwächt.

- Der Struktur satzeinleitendes es + sein-Kopula $+N P$ kann die Funktion Identifizierung zugeschrieben werden (Zifonun 2001), wobei diese Funktion nur in dieser Konstellation zustande kommt (vgl. *Er war Peter. vs. Er war sehr schnell.).

- Es ist möglich, einen Zusammenhang zwischen dieser spezifischen (weil funktionalen) Verbindung von es mit der Kopula und Thetizitätsmarkierung herzustellen (vgl. die Belege (53) bis (55) und die Anmerkungen dazu in 2.2.3), wobei Thetizität ein grundsätzlich auf das nicht-phorische es zugeschnittenes Konzept ist (s. Scheibl 2000). 
Die morphosyntaktische und funktionale Geprägtheit dieser Struktur rückt sie meiner Meinung nach in die Nähe nicht-phorischer es-Typen.

(d) Es ist so: Das es kann unter vier Aspekten interpretiert werden:

- als summierende Anapher zum vorangehenden Textteil

- als Korrelat

- als Bestandteil einer Extrapositionskonstruktion mit pragmatischer Funktion im Diskurs (Rahmung und Ankündigung salienter Informationen), vgl. Günthner (2009)

- als eine Art Spaltsatz, vgl. es ist Peter, der / es ist so, daß

Im Grunde genommen sind alle vier Optionen legitim sind. Synchron ist es eine Frage der Gewichtung, welche Analyse man zugrunde legt. Sprachhistorisch gesehen halte ich eine Deutung für naheliegend, die von der Pragmatisierung eines korrelathaltigen Matrixsatzes ausgeht. Dabei bleibt die Spaltsatz-Analyse, zumindest funktional, m.E. immer noch gültig.

Die Beispiele unter (a) bis (d) zeigen, dass zwischen den unterschiedlichen es-Typen Beziehungen unterschiedlichster Art hergestellt werden können. In der vorliegenden Arbeit wurde der Versuch unternommen, zumindest einige Möglichkeiten zur Beschreibung des esGesamtbildes und zur Aufdeckung von Zusammenhängen zwischen einzelnen es-Typen aufzuzeigen. 


\section{Literaturverzeichnis}

\subsection{Quellentexte}

Bräker, Ulrich (1789/1985): Lebensgeschichte und natürliche Abenteuer des Armen Mannes im Tockenburg. Berlin (= B).

Consentius, Ernst (Hrsg.) (1915): Meister Johann Dietz des Großen Kurfürsten Feldscher und Königlicher Hofbarbier (Mein Lebenslauf). Nach der alten Handschrift in der Königlichen Bibliothek zu Berlin zum ersten Male in Druck gegeben. Ebenhausen bei München (=MD).

Eckhardt, Wilhelm / Klingelhöfer, Helmut (Hrsg.) (1998): Bauernleben im Zeitalter des Dreißigjährigen Krieges. Die Stausenbacher Chronik des Caspar Preis 1636-1667. Mit einer Einführung von Gerhard Menk. Marburg (Beiträge zur hessischen Geschichte 13) $(=\mathrm{BL})$.

Fix, Ulla / Barth, Dagmar (2000): Sprachbiographien. Sprache und Sprachgebrauch vor und nach der Wende von 1989 im Erinnern und Erleben von Zeitzeugen aus der DDR. Inhalte und Analysen narrativ-diskursiver Interviews. Frankfurt a.M. (Leipziger Arbeiten zur Sprach- und Kommunikationsgeschichte 7) (=SB).

Gabriel, Christina (1800/1999): Meine Lebensgeschichte: die autobiographische Lebensbeschreibung einer Dienstmagd, Näherin und Hebamme im Herzogtum Westfalen um das Jahr 1800. Dokumentation eines bewegten Frauenlebens mit zeitgeschichtlichen Hintergründen. Bearb. von Christiane Vollmer. Hrsg. im Auftr. der Stadt Arnsberg, Stadtarchiv und des Arnsberger Heimatbundes e.V. von Michael Gosmann. Arnsberg (= DM).

Güntzer, Augustin (1657/2002): Kleines Biechlin von meinem gantzen Leben. Die Autobiographie eines Elsässer Kannengießers aus dem 17. Jahrhundert. Hrsg. Von Fabian Brändle und Dominik Sieber. Köln (Selbstzeugnisse der Neuzeit 8) (= G).

Jägerbursch (1751-1800): Beruflicher Werdegang und Lebensgeschichte eines Jägerburschen. Transkribiert von Ulrike Bauer-Both (= J).

Koralek, Ottilie (1851-1900): Lamentatio intermissa I. Tagebucharchiv Emmendingen. Unveröffentlichte Transkription (Hollmann) (=K).

Lächele, Rainer (Hrsg.) (1997): Hans Ludwig Nehrlich. Erlebnisse eines frommen Handwerkers im späten 17. Jahrhundert. Tübingen (Hallesche Quellenpublikationen und Repertoiren 1) (= NE).

Laible, Johann Gottlieb (1801-1850): Briefe an seinen Bruder Jakob. Tagebucharchiv Emmendingen. Unveröffentlichte Transkription (Hollmann) (= L).

Schiele, Lucie (1851-1900): Briefe an Alexander Brückner (1859-1860). Tagebucharchiv Emmendingen. Unveröffentlichte Transkription (Hollmann) (= SCH). 
Spinler, Karoline von / Ritter von Neupauer, Karl (1901-1950): Liebesbriefe des Braut- und Ehepaares von Neupauer. Sammlung Frauennachlässe, Institut der Geschichte der Universität Wien. NL 31. (= LB)

Tschofen, Bernhard (Hrsg.) (1996): Regina Lampert. Die Schwabengängerin. Erinnerungen einer jungen Magd aus Voralrberg 1864-1874. Zürich (= SCHG)

\subsection{Sekundärliteratur}

Admoni, Wladimir (1976): Es handelt sich um es. Zur gegenwärtigen Lage in der Grammatiktheorie. In: Wirkendes Wort 4, 219-227.

Ágel, Vilmos (1997). Reflexiv-Passiv, das (im Deutschen) keines ist. Überlegungen zu Reflexivität, Medialität, Passiv und Subjekt. In: Dürscheid, Christa / Ramers, Karl Heinz / Schwarz, Monika (Hrsg.): Sprache im Fokus. Festschrift für Heinz Vater zum 65. Geburtstag. Tübingen, 147-187.

Ágel, Vilmos (2000a): Syntax des Neuhochdeutschen bis zur Mitte des 20. Jahrhunderts. In: Besch, Werner / Betten, Anne / Reichmann, Oskar / Sonderegger, Stefan (Hrsg.): Sprachgeschichte. Ein Handbuch zur Geschichte der deutschen Sprache und ihrer Erforschung. 2. Teilband. Berlin / New York, 1855-1903.

Ágel, Vilmos (2000b): Valenztheorie. Tübingen.

Ágel, Vilmos (2003): Prinzipien der Grammatik. In: Lobenstein-Reichmann, Anja / Reichmann, Oskar (Hrsg.): Neue historische Grammatiken. Zum Stand der Grammatikschreibung historischer Sprachstufen des Deutschen und anderer Sprachen. Tübingen, 1-47 (= RGL 243).

Ágel, Vilmos (2003): Wort- und Ausdrucksvalenz(träger). In: Cornell,Alan / Fischer, Klaus / Roe, Ian F. (Hrsg.): Valency in Practice / Valenz in der Praxis. Oxford, 17-36.

Ágel, Vilmos (2004): Phraseologismus als (valenz)syntaktischer Normalfall. In: Steyer, Kathrin (Hrsg.): Wortverbindungen - mehr oder weniger fest. Berlin / New York, 65-87 (= IDS Jahrbuch 2003).

Ágel, Vilmos (2007): Was ist ,grammatische Aufklärung' in einer Schriftkultur? Die Parameter ,Aggregation“ und ,Integration“. In: Feilke, Helmuth / Knobloch, Clemens / Völzing, Paul-Ludwig (Hrsg.): Was heißt linguistische Aufklärung? Sprachauffassungen zwischen Systemvertrauen und Benutzerfürsorge. Heidelberg, 3957 (Wissenschaftskommunikation 1).

Ágel, Vilmos / Hennig, Mathilde (Hrsg.) (2006): Grammatik aus Nähe und Distanz. Theorie und Praxis am Beispiel von Nähetexten 1650-2000. Tübingen.

Ágel, Vilmos / Diegelmann, Carmen (2010): Theorie und Praxis der expliziten Junktion. In: Ágel, Vilmos / Hennig, Mathilde (Hrsg.): Nähe und Distanz im Kontext variationslinguistischer Forschung. Berlin / New York, 345-393.

Ariel, Mira (1990): Accessing Noun-Phrase Antecendents. London / New York. 
Asher, Nicholas (1993): Reference to Abstract Objects in Discourse. Dordrecht (= Studies in linguistics and philosophy 50).

Askedal, John Ole (1990): Zur syntaktischen und referentiell-semantischen Typisierung der deutschen Pronominalform es. In: Deutsch als Fremdsprache 27, 213-225.

Auer, Peter (1998): Zwischen Parataxe und Hypotaxe: ,abhängige Hauptsätze“ im gesprochenen und geschriebenen Deutsch. In: ZGL 26, 284-307.

Auer, Peter (2000): On line-Syntax. In: Sprache und Literatur 85 (31), 43-56.

Back, Michael (1995): Ich weiß nicht, was soll „ES“ bedeuten. In: DS 2, 147-148.

Behaghel, Otto (1923): Deutsche Syntax. Eine geschichtliche Darstellung. Band I. Die Wortklassen und Wortformen. A. Nomen, Pronomen. Heidelberg.

Behaghel, Otto (1928): Deutsche Syntax. Eine geschichtliche Darstellung. Band III. Die Satzgebilde. Heidelberg.

Behrens, Heike (1999): Was macht Verben zu einer besonderen Kategorie im Spracherwerb? In: Meibauer, Jörg / Rothweiler, Monika (Hrsg.): Das Lexikon im Spracherwerb. Tübingen / Basel, 32-50.

Bethke, Inge (1990): Der, die, das als Pronomen. München (= Studien Deutsch 11).

Brugmann, Karl (1917): Der Ursprung des Scheinsubjektes ,es‘ in den germanischen und romanischen Sprachen. Leipzig.

Bühler, Karl (1965): Sprachtheorie. Die Darstellungsfunktion der Sprache. 2. Aufl. Stuttgart.

Bybee, Joan L. / Perkins, Revere / Pagliuca, William (1994): The Evolution of Grammar. Tense, Aspect, and Modality in the Languages of the World. Chicago / London.

Cardinaletti, Anna (1990): Es, pro and sentential arguments in German. In: LB 126, 135 165.

Cho, Kyun (1975): Die Konstruktionen mit "es" im Neuhochdeutschen. Freiburg (Schweiz).

Consten, Manfred / Schwarz-Friesel, Monika (2007): Anapher. In: Hoffmann, Ludger (Hrsg.): Deutsche Wortarten. Berlin / New York, 265-292.

Cornish, Francis (2005): Degrees of indirectness: two types of implicit referents and their retrieval via unaccented pronuns. In: Branco, António / McEnery, Tony / Mitkov, Ruslan (Hrsg.): Anaphora processing. Linguistic, cognitive and computational modelling. Amsterdam, 199-220.

Croft, William (2002): Radical Construction Grammar. Syntactic Theory in Typological Perspective. Oxford.

Csányi, Vilmos (1999): Az emberi természet [Die menschliche Natur]. Budapest.

Czicza, Dániel (2003): Theoretische Überlegungen zu einer möglichen Neuorientierung in der es-Forschung. In: Németh, Attila (Hrsg.): Linguistische Beiträge ungarischer Nachwuchsgermanisten. Referate der I. Linguistischen Tagung ungarischer 
Nachwuchsgermanisten an der Universität Veszprém vom 28.-29. März 2003. Veszprém / Wien, 25-49.

Czicza, Dániel (2007): ES-constructions. In: Balaskó, Mária / Szatmári, Petra (Hrsg.): Sprach- und literaturwissenschaftliche Brückenschläge. Vorträge der 13. Jahrestagung der GESUS in Szombathely, 12.-14. Mai 2004, München, 305-318.

Dal, Ingerid (1966): Kurze deutsche Syntax auf historischer Grundlage. Tübingen.

Dalmas, Martine (1996): Die sogenannten Nebensatz-"Korrelate". Oder: Vertretungen nur als Mittel zur Rettung von Leerstellen? In: Pérenner, Marie-Hélène (Hrsg.): Pro-Formen des Deutschen. Tübingen, 23-34 (= Eurogermanistik 10).

Diewald, Gabriele (1991): Deixis und Textsorten im Deutschen. Tübingen (= RGL 118).

Diewald, Gabriele (1997): Grammatikalisierung. Eine Einführung in Sein und Werden grammatischer Formen. Tübingen.

Diewald, Gabriele (2006): Context types in grammaticalization as constructions. In: Schönefeld, Doris (Hrsg.): Constructions. Special Volume 1: Constructions all over case studies and theoretical implications $(=$ http://www.constructionsonline.de/articles/specvoll/, Stand: 23.9.2008).

Duden-Grammatik (2005) $=$ Duden. Die Grammatik. 7. Aufl. Mannheim / Leipzig / Wien / Zürich.

Dürscheid, Christa (1995): Passivische Konstruktionen im Deutschen und Ungarischen. In: Jahrbuch der ungarischen Germanistik. Budapest, 109-126.

Ebert, Rober Peter (1978): Historische Syntax des Deutschen. Stuttgart.

Ebert, Robert Peter (1993): Syntax. In: Reichmann, Oskar / Wegera, Klaus-Peter (Hrsg.): Frühneuhochdeutsche Grammatik. Tübingen, 313-485.

Ehlich, Konrad (2007): Sprache und sprachliches Handeln. Band 2: Prozeduren des sprachlichen Handelns. Berlin / New York.

Eisenberg, Peter (2006): Grundriss der deutschen Grammatik. Band 2. Der Satz. Stuttgart.

Erben, Johannes (1979): Zur Multivalenz von es im Neuhochdeutschen und im Sprachstil der Grimmschen Märchen. In: Wirkendes Wort 6, 384-391.

Eroms, Hans Werner (2000): Syntax der deutschen Sprache. Berlin / New York.

Eroms, Hans-Werner (2004): Die Ausweitung der Dependenzzone. In: Czicza, Dániel / Hegedüs, Ildikó / Kappel, Péter / Németh, Attila (Hrsg.): Wertigkeiten, Geschichten und Kontraste. Festschrift für Péter Bassola zum 60. Geburtstag. Szeged, 151-165.

Faucher, Eugène (1996): Umfunktionierte Pronomina. In: Pérenner, Marie-Hélène (Hrsg.): Pro-Formen des Deutschen. Tübingen, 49-63 (= Eurogermanistik 10).

Feilke, Helmuth (1996): Sprache als soziale Gestalt. Ausdruck, Prägung und die Ordnung der sprachlichen Typik. Frankfurt a.M.. 
Feilke, Helmuth (1998): Idiomatische Prägung. In: Barz, Irmhild / Öhlschläger, Günther (Hrsg.): Zwischen Grammatik und Lexikon. Tübingen, 69-80 (= Linguistische Arbeiten 390).

Feilke, Helmuth (2003): Textroutine, Textsemantik und sprachliches Wissen. In: Linke, Angelika / Ortner, Hanspeter / Portmann-Tselikas, Paul R. (Hrsg.): Sprache und mehr. Ansichten einer Linguistik der sprachlichen Praxis. Tübingen, 209-231.

Feldman, Jerome / Dodge, Ellen / Bryant, John (2010): Embodied construction grammar. In: Heine, Bernd / Narrog, Heiko (Hrsg.): Oxford Handbook of Linguistic Analysis. Oxford, 111-137.

Fillmore, Charles J. (1989): Grammatical construction theory and the familiar dichotomies. In: Dietrich, Rainer / Graumann, Charles F. (Hrsg.): Language processing in social context. Amsterdam, 17-38.

Fillmore, Charles J. / Kay, Paul / O’Connor, M.C. (1988): Regularity and idiomaticity in grammatical constructions: The case of Let Alone. In: Language 64/3, 501-538.

Fischer, Kerstin / Stefanowitsch, Anatol (2006): Konstruktionsgrammatik I: Von der Anwendung zur Theorie. Tübingen.

Fischer, Kerstin / Stefanowitsch, Anatol (2008): Konstruktionsgrammatik II: Von der Konstruktion zur Grammatik. Tübingen.

Fischer, Klaus (2009): Cleft sentences: Form, function and translation. In: Journal of Germanic Linguistics 21.2, 167-192.

Fourquet, Jean (1970): Prolegomena zu einer deutschen Grammatik. Düsseldorf (= Sprache der Gegenwart 7).

Fried, Mirjam / Östman, Jan-Ola (2004): Construction Grammar: A thumbnail sketch. In: Fried, Mirjam / Östman, Jan-Ola (Hrsg.): Construction Grammar in a cross-language perspective. Amsterdam / Philadelphia, 11-86 (= Constructional Approaches to Language 2).

Goldberg, Adele E. (1995): Constructions: A Construction Grammar Approach to Argument Structure. Chicago.

Goldberg, Adele E. (1996): Making one's way through the data. In: Shibatani, Masayoshi / Thompson, Sandra A. (Hrsg.): Grammatical constructions. Their form and meaning. Oxford, 29-55.

Goldberg, Adele E. (2006): Constructions at work: The nature of generalization in language. Oxford.

Grice, H. Paul (1975): Logik und Konversation. In: Hofmann, Ludger (Hrsg.) (2000): Sprachwissenschaft. Ein Reader. 2. Aufl. Berlin / New York, 163-183.

Große, Rudolf (1990): Funktionen des Pronomens iz im Althochdeutschen. In: Betten, Anne (Hrsg.): Neuere Forschungen zur historischen Syntax des Deutschen. Referate der internationalen Fachkonferenz Eichstätt 1989. Tübingen, 29-39.

Günthner, Susanne (2009): Extrapositionen mit es im gesprochenen Deutsch. In: ZGL 37, $15-46$. 
Günthner, Susanne / Imo, Wolfgang (Hrsg.) (2006): Konstruktionen in der Interaktion. Berlin / New York.

Haider, Hubert (1993): Deutsche Syntax - generativ. Tübingen.

Harweg, Roland (1979): Pronomina und Textkonstitution. 2. Aufl. München.

Hegedüs, Ildikó (2007): Wie kann Nähesprache diachron untersucht werden? Problemanalyse am Beispiel der Korrelate von Subjekt- und Objektsätzen. In: Ágel, Vilmos / Hennig, Mathilde (Hrsg.): Zugänge zur Grammatik der gesprochenen Sprache. Tübingen, 245-272.

Helbig, Gerhard / Buscha, Joachim (2001): Deutsche Grammatik. Ein Handbuch für den Ausländerunterricht. Leipzig.

Hennig, Mathilde (2006): Grammatik der gesprochenen Sprache in Theorie und Praxis. Kassel.

Hens, Gregor (1996): (jm) (einen Brief) schreiben: Zur Valenz in der Konstruktionsgrammatik. In: LB 164, 334-356.

Hentschel, Elke (2003): Es war einmal ein Subjekt. In: Linguistik online 13, 137-160.

Himmelmann, Nikolaus P. (1997): Deiktikon, Artikel, Nominalphrase. Zur Emergenz syntaktischer Struktur. Tübingen (= Linguistische Arbeiten 362).

Hoeing, Robert G. (1994): Empty, expletive, and missing subjects in German. New York.

Hopper, Paul (2000): Prototype or family resemblance? Grammatical constructions and their discourse origins. Reproduced by LAUD, Essen (= Series A, General and Theoretical Paper No. 508).

Ickler, Irene (1990): Kasusrahmen und Perspektive. Zur Kodierung von semantischen Rollen. In: DS 18, 1-37.

IDS-Grammatik (1997) = Zifonun, Gisela / Hoffmann, Ludger / Strecker, Bruno et al.: Grammatik der deutschen Sprache. 3 Bde. Berlin / New York.

Imo, Wolfgang (2007a): Construction Grammar und Gesprochene-Sprache-Forschung: Konstruktionen mit zehn matrixsatzfähigen Verben im gesprochenen Deutsch. Tübingen (= RGL 275).

Imo, Wolfgang (2007b): Zur Anwendung der Construction Grammar auf die gesprochene Sprache - der Fall ,ich mein(e)“. In: Ágel, Vilmos / Hennig, Mathilde (Hrsg.): Zugänge zur Grammatik der gesprochenen Sprache. Tübingen, 3-35.

Jacobs, Joachim (2009): Valenzbindung oder Konstruktionsbindung? Eine Grundfrage der Grammatiktheorie. In: ZGL 37/3, 490-513.

Jaeger, Christoph (1992): Probleme der syntaktischen Kongruenz: Theorie und Normvergleich im Deutschen. Tübingen.

Jäger, Ludwig (2003): Erkenntnisobjekt Sprache. Probleme der linguistischen Gegenstandskonstitution. In: Linke, Angelika / Ortner, Hanspeter / Portmann-Tselikas, Paul R. (Hrsg.): Sprache und mehr. Ansichten einer Linguistik der sprachlichen Praxis. Tübingen, 67-99. 
Kálmán, László (2001): Konstrukciós nyelvtan [Konstruktionsgrammatik]. Budapest (= Segédkönyvek a nyelvészet tanulmányozásához VIII).

Kemme, Hans-Martin (1979): Der Gebrauch des „es“ im Deutschen. Eine Darstellung für den Unterricht an Ausländer. München.

Kemmerling, Andreas (1991): Implikatur. In: Stechow, Arnim von / Wunderlich, Dieter (Hrsg.): Semantics / Semantik. An International Handbook of Contemporary Research / Ein internationales Handbuch zeitgenössischer Forschung. Berlin / New York, 319-333.

Knobloch, Clemens (2009): Einladung und Einleitung. Einleitender Text im ZGLSonderheft mit dem Themenschwerpunkt Konstruktionsgrammatik. In: ZGL 37/3, 385402 .

Koch, Peter / Oesterreicher, Wulf (1985): Sprache der Nähe - Sprache der Distanz. Mündlichkeit und Schriftlichkeit im Spannungsfeld von Sprachtheorie und Sprachgeschichte. In: Romanistisches Jahrbuch 36, 15-43.

Lakoff, George (1987): Women, Fire, and Dangerous Things. What Categories Reveal about the Mind. Chicago / London.

Langacker, Ronald (2007): Constructing the meanings of personal pronouns. In: Radden, Günter / Köpcke, Klaus-Michael / Berg, Thomas / Siemund, Peter (Hrsg.): Aspects of meaning construction. Amsterdam, 171-187.

László, Sarolta (1988): Mikroebene. In: Mrazović, Pavica / Teubert, Wolfgang (Hrsg.): Valenzen im Kontrast. Ulrich Engel zum 60. Geburtstag. Heidelberg, 218-233.

Latour, Bernd (1981): Zur Fakultativität des Pronomens es als Korrelat satzförmiger Ergänzungen. In: Jahrbuch Deutsch als Fremdsprache 7, 240-253.

Lehmann, Christian (1988): Towards a typology of clause linkage. In: Haiman, John / Thompson, Sandra A. (Hrsg.): Clause combining in discourse and grammar. Proceedings of a conference at Rensselaerville Institute, Albany, N.Y. Amsterdam, 181225 (= Typological Studies in Language 18).

Lenerz, Jürgen (1985): Zur Theorie syntaktischen Wandels: Das expletive es in der Geschichte des Deutschen. In: Abraham, Werner (Hrsg.): Erklärende Syntax des Deutschen. Tübingen, 99-136.

Lenz, Alexandra (2007): Zur Grammatikalisierung von geben im Deutschen und Lëtzebuergeschen. In: ZGL 35, 52-82.

Leys, Odo (1979): Zur Systematisierung von ES. In: DS 7, 28-34.

Maienborn, Claudia (2007): Das Zustandspassiv. Grammatische Einordnung Bildungsbeschränkung - Interpretationsspielraum. In: ZGL 35, 83-114.

Marx-Moyse, Jeanine (1983): Untersuchungen zur deutschen Satzsyntax. ,Es“ als vorausweisendes Element eines Subjektsatzes. Wiesbaden.

Michaelis, Laura A. (2010): Sign-based construction grammar. In: Heine, Bernd / Narrog, Heiko (Hrsg.): Oxford Handbook of Linguistic Analysis. Oxford, 139-158. 
Michaelis, Laura A. / Ruppenhofer, Josef (2001): Beyond alternations: a constructional model of the German applicative pattern. Stanford, CA.

Näß1, Susanne (1996): Die „okkasionellen Ereignisverben” im Deutschen: synchrone und diachrone Studien zu unpersönlichen Konstruktionen. Frankfurt a.M.

Newman, John (1998): The Origin of the German es gibt construction. In: Newman, John (Hrsg.): The Linguistics of Giving. Amsterdam / Philadelphia, 307-325.

Östman, Jan-Ola (2005): Construction discourse. A prolegomenon. In: Östman, Jan-Ola / Fried, Mirjam (Hrsg.): Construction grammars. Cognitive grounding and theoretical extensions. Amsterdam / Philadelphia, 121-144.

Östman, Jan-Ola / Fried, Mirjam (2004): Historical and intellectual background of Construction Grammar. In: Fried, Mirjam / Östman, Jan-Ola (Hrsg.): Construction Grammar in a cross-language perspective. Amsterdam / Philadelphia, 1-10 (= Constructional Approaches to Language 2).

Pause, Peter E. (1991): Anaphern im Text. In: Stechow, Arnim von / Wunderlich, Dieter (Hrsg.): Semantics / Semantik. An International Handbook of Contemporary Research / Ein internationales Handbuch zeitgenössischer Forschung. Berlin / New York, 548-560.

Polenz, Peter von (1985): Deutsche Satzsemantik. Grundbegriffe des Zwischen-den-ZeilenLesens. Berlin.

Polenz, Peter von (2000): Deutsche Sprachgeschichte vom Spätmittelalter bis zur Gegenwart. Band I: Einführung, Grundbegriffe, 14. bis 16. Jahrhundert. Berlin / New York.

Pütz, Herbert (1975): Über die Syntax der Pronominalform „es“ im modernen Deutsch. Tübingen.

Sandberg, Bengt (1998): Zum „es“ bei transitiven Verben vor satzförmigem Akkusativobjekt. Tübingen.

Scheibl, György (2000): Zur Unterscheidung thetisch-kategorisch in deutschen esKonstruktionen. In: DS 28, 372-384.

Schmidt, Ulrich A. (1987): Impersonalia, Diathesen und die deutsche Satzgliedstellung. Bochum.

Schwarz, Monika (1997): Anaphern und ihre diversen Antezedenten: Korferenz und Konsorten. In: Dürscheid, Christa / Ramers, Karl Heinz / Schwarz, Monika (Hrsg.): Sprache im Fokus. Festschrift für Heinz Vater zm 65. Geburtstag. Berlin / New York, 445-455.

Schwarz, Monika (2000): Indirekte Anaphern in Texten. Studien zur domänengebundenen Referenz und Kohärenz im Deutschen. Tübingen.

Schwarz-Friesel, Monika / Consten, Manfred / Marx, Konstanze (2004): Semantische und konzeptuelle Prozesse bei der Verarbeitung von Komplex-Anaphern. In: Pohl, Inge / Konerding, Klaus-Peter (Hrsg.): Stabilität und Flexibilität in der Semantik. Frankfurt a.M., 67-88. 
Seefranz-Montag, Ariane von (1995): Impersonalien. In: Jacobs, Joachim /Stechow, Arnim von / Sternefeld, Wolfgang / Vennemann, Theo (Hrsg.): Syntax. Ein internationales Handbuch zeitgenössischer Forschung. Berlin / New York, 1277-1287.

Smith, Michael B. (2002): The Polysemy of German es, Iconicity, and the Notion of Conceptual Distance. In: Cognitive Linguistics 13, 67-112.

Smith, Michael B. (2005): The Conceptual Structure of German Impersonal Constructions. In: Journal of Germanic Linguistics 17/2, 79-140.

Starke, Michal (1996): Germanische und romanische Pronomina: stark - schwach - klitisch. In: Lang, Ewald / Zifonun, Gisela (Hrsg.): Deutsch - typologisch. Jahrbuch 1995 des Instituts für deutsche Sprache. Berlin / New York, 405-428.

Stein, Stephan (1995): Formelhafte Sprache: Untersuchungen zu ihren pragmatischen und kognitiven Funktionen im gegenwärtigen Deutsch. Frankfurt a.M..

Stein, Stephan (2004): Formelhaftigkeit und Routinen in mündlicher Kommunikation. In: Steyer, Kathrin (Hrsg.): Wortverbindungen - mehr oder weniger fest. Berlin / New York, 262-289 (= IDS-Jahrbuch 2003).

Szakmary, Corinna (2002): Personenreferenz und Textplanung. Eine textlinguistische Untersuchung über den Einfluss von Textplanungsprozessen auf die sprachliche Realisierung von Personenreferenzen. Frankfurt a.M.

Szatmári, Petra (1998): Es - und kein Ende. Zum nicht-phorischen es in passivisch interpretierbaren sich-lassen-Konstruktionen. In: Jahrbuch der ungarischen Germanistik, 225-242.

Traugott, Elisabeth Closs (2003): Constructions in grammaticalization. In: Joseph, Brian D. / Janda, Richard D. (Hrsg.): The Handbook of Historical Linguistics. Oxford, 624647.

Traugott, Elisabeth Closs (2008): Grammatikalisierung, emergente Konstruktionen und der Begriff der „Neuheit“. In: Fischer, Kerstin / Stefanowitsch, Anatol (Hrsg.): Konstruktionsgrammatik II: Von der Konstruktion zur Grammatik. Tübingen, 5-32.

Ulvestad, Bjarne / Bergenholtz, Henning (1979): Es als Vorgreifer eines Objektsatzes. In: DS 7, 97-116.

Ulvestad, Bjarne / Bergenholtz, Henning (1983): Es als Vorgreifer eines Objektsatzes. Teil II. In: DS 11, 1-26.

van Hoek, Karen (1997): Anaphora and Conceptual Structure. Chicago / London (= Cognitive Theory of Language and Culture).

Vater, Heinz (2005): Referenz-Linguistik. München.

Vogel, Petra (2006): Das unpersönliche Passiv. Eine funktionale Untersuchung unter besonderer Berücksichtigung des Deutschen und seiner historischen Entwicklung. Berlin / New York.

Wegener, Heide (1999): Zum Bedeutungs- und Konstruktionswandel bei psychischen Verben. In: Wegener, Heide (Hrsg.): Deutsch kontrastiv. Typologisch-vergleichende Untersuchungen zur deutschen Grammatik. Tübingen, 171-210. 
Weinrich, Harald (1993): Textgrammatik der deutschen Sprache. Unter Mitarbeit von Maria Thurmair, Eva Breindl, Eva-Maria Willkop. Mannheim / Leipzig / Wien / Zürich.

Welke, Klaus (2002): Deutsche Syntax funktional. Perspektiviertheit syntaktischer Strukturen. Tübingen.

Welke, Klaus (2007): Das Zustandspassiv. Pragmatische Beschränkungen und Regelkonflikte. In: ZGL 35, 115-145.

Welke, Klaus (2009a): Valenztheorie und Konstruktionsgrammatik. In: ZGL 37/1, 81-124.

Welke, Klaus (2009b): Konstruktionsvererbung, Valenzvererbung und die Reichweite von Konstruktionen. In: ZGL 37/3, 514-543.

Willems, Klaas / Coene, Ann (2006): Satzmuster und die Konstruktionalität der Verbbedeutung. Überlegungen zum Verhältnis von Konstruktionsgrammatik und Valenztheorie. In: Sprachwissenschaft 31, 237-272.

ZGL-Sonderheft 37/3 Konstruktionsgrammatik (2009), hrsg. von Ágel, Vilmos / Feilke, Helmuth / Linke, Angelika / Lüdeling, Anke / Tophinke, Doris.

Zifonun, Gisela (1995): Minimalia grammaticalia: das nicht-phorische es als Prüfstein grammatischer Theoriebildung. In: DS 23, 39-60.

Zifonun, Gisela (2001): Grammatik des Deutschen im europäischen Vergleich: Das Pronomen. Teil I: Überblick und Personalpronomen. Mannheim.

Zifonun, Gisela (2007): Zur Grammatik des Deutschen im europäischen Vergleich. Das Pronomen. Teil IV: Indefinita im weiteren Sinne. Mannheim. (= amades 2007).

Zitterbart, Jussara Paranhos (2002a): Zur Mittelfeldfähigkeit des Korrelats es in Verbindung mit Subjektsätzen. In: Sprachwissenschaft 27, 149-195.

Zitterbart, Jussara Paranhos (2002b): Zur korrelativen Subordination im Deutschen. Tübingen (= Linguistische Arbeiten 464). 


\section{Anhang}

\subsection{Das Belegkorpus}

Das Belegkorpus beinhaltet nur diejenigen Belege aus meinem eigenen Korpus, die in der vorliegenden Arbeit besprochen werden, nicht aber solche aus der Fachliteratur. Die Belege werden nach Kapiteln geordnet und entsprechend ihrer Abfolge im Text angeführt.

\section{Kapitel 2}

Ich halte es also für unwahrscheinlich, daß dieses ständig geübte Vokabular nicht auch in der Familie auftauchte. Das geht wahrscheinlich gar nicht anders. ... Und es tauchte auch auf. (17) (SB)

Welche Unterschiede gab es zwischen der östlichen und der westlichen Mediensprache? (8) (SB)

\section{Kapitel 2.1}

Sobald ein Pferd von uns sturbe, welches gar oft geschahe, maßen wir gar keines behielten, tranchiereten sie es gleich und machten sich lustig dabei. (19) (MD)

es war eine Lust und Freude jeden Morgen das Auflesen unter den Bäumen und eine Lust, immer vom schönsten Obst zu essen. (17) (SCHG)

da werden die Pfingsten schöner sein, es werden vielleicht die Lieben aus Teplitz kommen. (5) (K)

Aber diese Bezeichnungen machen es ja eigentlich deutlich, wie man zu diesen Leuten steht (13) (SB)

Gerti [...] Bertha [...] Es sind halt überhaupt 2 wilde Kinder, wo sie das nur her haben?????? (1890) (LB)

〈Weisst Du, später, wenns Du einmal weisst, wie mans macht, musst Du allein die Gänse treiben. (9) (SCHG)

Und da finden wir in dem ausgestorbenen Haus: Speck, Butter, Mehl, Eier, Fleisch und alles gnung. So er auch wohl gewußt. Da ging es mit uns an ein Sieden und Braten, denn wir wurden gar knapp erhalten (7) (MD)

\section{Kapitel 2.2}

Der starke Zug nach den Mühlen hätte mich schier ertränket und ersauft, wann nicht mein Berkaner Mantel Wind gefasset und mich oben erhalten. Unter der großen Arbeit, wieder heraus zu kommen, war nichts als: JEsus, JEsus, ach JEsus, mein GOtt, hilf mir! mein Gedanke. Endlich war ein Stück 
Bauholz mit eingefroren. Daran half ich mir wieder raus. Da war kein Mensch, der es gesehen oder mir helfen können. (10) (MD)

daß es der ewige Schlaf ist, den sie schläft, daß sie - kaum erwachen werde. Und so war es gekommen. Sie - ist um $6 \mathrm{Uhr}$ eingeschlafen, hat bis Mitternacht noch geathmet, u. ist dann verschieden. (24) (K)

als aber ein bar stunden vergingen, und ich mich den lieben Gott befohlen mit gebet, und ich mich drein ergeben, ich württe die nacht tauren müßen, endlich hörede etwas gantz dunckel bochen, ich lag aber stille, es bocht noch 2 oder 3 mal, so dachte ich, es were ein gespenst, endlich als es nicht wolte auff hören, und recht starck anschlug, so kroch ich heraus, suchte die treppe und kroch rund um biß ich nauf kam. (26) (NE)

Endlich wie es bald zweÿ wolte schlagen das auch der Herr Graff lang weg war (28) (NE)

Endlich entschloß ich mich, selber eins zu bauen, und sagte es meiner Schönen. Sie war's zufrieden, und bot mir wieder Geld dazu an. (11) (B)

In dem obbeschriebenem Jahr ist das Obs an den Bä[u]men gantz verdorben, das wir in unseren Garten nicht ein Hutt voll bekomen haben, es seyen gewest Äpfel oder Birn. (31) (BL)

durch seine ränke und gedungene guten freunde Brachte er Es würklich so weit beij seinem Vater | das er Ihn zum stamherrn machte (4) (DM)

die zeit verstrich, Es kahm kein schwager und kein kaufbrif (8) (DM)

Als ich nun zu ihnen kam, und mich als Deserteur angab, nahmen sie mir das Gewehr ab, unterm Versprechen, mir's nachwerts schon wieder zuzustellen. (4) (B)

Sophiechen ist confirmirt $\mathrm{u}$. ich habe mein Theil dabei geweint, wie viel auch Deiner gedacht $u$. gefunden, daß wir keineswegs so gesinnt sind wie wir es sein sollten (114) $(\mathrm{SCH})$

Es ist auch $\mathrm{Zu}$ verstehen, das mann auf solchen botten, welcher guth und drachtbar ist, keine weig oder natel holtzer, geren zieht, es müste dan seine besontere ursach haben (89) (J)

\section{Kapitel 2.2.1}

Sie muß fest von seine Treue u. Liebe überzeugt sein, denn es ist ein ernstes Verhältniß, sie corespondiren mit einander $(13)(\mathrm{K})$

$\mathrm{Zu}$ dem End kauft' ich im Eggberg, wo meine Dulcinee daheim war, etwas Salpetererde, und zugleich ihres Vaters Gaden - ihr zu lieb viel zu theuer; denn es war fast verloren Geld (10) (B)

Der Mörder ward ertapt, gefangen genomen, nach Marpurg uffs Schloß geführt. Was nun es vor ein Außgang wird gewinen, das wird die Zeit geben. (32) (BL)

Sie zogen fur den Berg, doch heimlicherweise, und wolten in einnemen, aber es wolt innen nicht glücken. (12) (BL) 
wenn ich ernsthaft krank wäre würde ich nach Salzburg fahren, dort weiß ich doch 2 empfohlene Ärzte- Aber es ist nichts ernsthaftes nur ein bisl hergenommen hat mich das alles, ich liege halt viel herum. (1154) (LB)

Ich erschrak und lauschte durchs schlüßelloch. und sahe, das sein bedienter | welcher ein sehr verschmitzer kerll war | vor Ihm auf den knien lag und Ihn mit aufgehobnen händen baht | von müller abzulaßen | es wäre sonst sein unglük. (3) (DM)

\section{Kapitel 2.2.2}

Sie zogen fur den Berg, doch heimlicherweise, und wolten in einnemen, aber es wolt innen nicht glücken. Es war ein uberauß ungestümige Nacht von Regen und Wind, von Brausen und Sausen, als in langer Zeitt nicht gewesen ist. (12) (BL)

Die Statt ward gantz und zumal außgeplundert, die arme Burgersleuth musten alle mit letigen Händen davon, konden nicht behalten, was sie am Leibe hatten. Ach Gott, der ellende Zeit. Der Oberste Königsmargk bleib uff Omeneburg ligen die sex Wochen, so lang das Läger wehret. Es war der schwedische Jenneral genant Frangel und war Jenneral Bönighaußen. Es waren die Hessen der selbige Jenneral genant Johan Gisso. [S. 59] Es war ein sehr grosses Volk, das sichs zu verwundern war, und war eben im Somer von S. Johainstag bis nach Jacobi. Ja, es war noch nicht gnung. (17) (BL)

Gerade von meiner Verheurathung an wollt' ich mit nichts geringerm beginnen, als - der Welt völlig abzusagen, und das Fleisch mit allen seinen Gelüsten zu kreutzigen. Aber o ich einfältiger Mensch! Was es da für ein Gewirre und für Widersprüche in meinem Innwendigen absetzte. (17) (B)

Es wollte mir aber kein Essen zu Leibe. (Es kame mir aber hernach zustatten, als die Not größer ward.) (23) (MD)

Was ist eigentlich passiert, wenn man diese Angepaßtheit durchbrochen hat? Ist es nicht verstanden worden, ist es reglementiert worden? (16) (SB)

Der einte war, daß ich noch viel zu wenig Holz hatte, ungeachtet Mstr. Brunner mir gesagt, es sey genug, und es erst itzt einsah, als er an die oberste oder Firstkammer kam. (12) (B)

Sophiechen ist confirmirt $u$. ich habe mein Theil dabei geweint, wie viel auch Deiner gedacht $u$. gefunden, daß wir keineswegs so gesinnt sind wie wir es sein sollten (114) (SCH)

Das Korn fängt an zu grünen und zu wachsen, es wird überall gepflügt mit Ochsen oder Pferde, gespannt vor dem Pflug. (10) (SCHG)

(Schau, ich bring Dir ein kleines Mädchen, nimms mit in die Stube und diesen Mann, komme nach.〉 (7) (SCHG)

Da habe ich noch nichts unternommen, weil ich durch das schlechte Wetter und mancherlei verhindert war nach Maria Grün zu hole. Sollte es aber schön bleiben so habe ich es für morgen Nach- // mittag vor. (2442) (LB) 
Den Bub will ich dann in Ailingen dem Lehrer Grossmann übergeben, es ist mir am Weg. (6) (SCHG)

\section{Kapitel 2.2.3}

also musten die Kayserischen weichen und musten inen das Schloß lassen, dann es war im Winter. (21) (BL)

als ich zurückkam, schlüpfte ich davon in mein Zimmer und setzte mich hin und las Deine Briefe durch und da ward's als ob Du mit mir sprechen würdest so lieb, so gut. Wenn's doch wieder einmal sein könnte! (1529) (LB)

«Je gelt! deine Anne ist auch verplempert; dein Vetter Michel war so glückselig, und sie hat schon ein Kind». - Das fuhr mir ja durch Mark und Bein; indessen ließ ich's den argen Unglückboten nicht merken (7) (B)

Gibt es heute immer noch ähnlich viele Wörter mit Konnotationen oder ist es vielleicht nicht mehr so notwendig, in der heutigen Sprache solche Wörter zu verwenden? (12) (SB)

meine gnädige frau | die Es gemerkt hate $\mid$ das müller alles aufboht $\mid$ hielt mich in der größten stränge (2) $(\mathrm{DM})$

Wie es noch weider wird abgehen und die liebe Frucht wird inbracht werden, das weiß der liebe, getreue Gott und mir werden es erfahren. (26) (BL)

Endlich entschloß ich mich, selber eins zu bauen, und sagte es meiner Schönen. (11) (B)

doch seit Du mich gebeten, ich solle mich schonen, halt ich's für meine Pflicht Alles zu vermeiden, was mir schaden kann. (99f.) ( $\mathrm{SCH})$

u. doch fehlte mir die Demuth dazu; kurz, ich möchte verzweifeln, doch darf ich es ja nicht (115) $(\mathrm{SCH})$

In dieser bangen Nacht desertirten viele; neben andern auch Bruder Bachmann. Für mich wollt' es sich noch nicht schicken, so wohl's mir sonst behagt hätte. (1) (B)

Die 1. Gabi bekam von der Großmutter aus Chr. ein goldenes Armband, vielmehr Geld um es sich zu kaufen. (10) (K)

Gott der Herr walte über euren kleinen, u. seid überzeugt, wenns erhalten werde, so wird er erhalten. (14) (L)

Da ging es mit uns an ein Sieden und Braten, denn wir wurden gar knapp erhalten; wir buken so viel Pfannkuchen, Eierfladen \{et cetera\} und schleppten's mit heim, daß wir wohl vierzehen Tage davon essen konnten. (7) (MD)

Kirschen dürfen wir essen, so viel mir wollen. Nur müssens wir selbst pflücken. (11) (SCHG)

Der Grabstein sieht jetzt geputzt schön aus, der Steinmetz sagt es wäre Marmor, (1954) (LB) 
fuhren bald vor bald hinter uns in die Erde, daß Stein und Rasen hoch in die Luft sprang - bald mitten ein, und spickten uns die Leuthe aus den Gliedern weg, als wenn's Strohhälme wären. (2) (B)

Der Oberknecht, es war der älteste Sohn aus erster Ehe, sagte mir die Köchin, steht auf und betete laut mit allen zusammen ein ziemlich langes Tischgebet, katholisch. (10) (SCHG)

Doch ist sie nur so schön $u$. ich bin es nicht. (108) (SCH)

\section{Kapitel 2.3.2}

ich sagte kein wort und verlangte keine Erdbiren $\mid$ aber bitter war es mir, mich auf diese art von meiner mutter behandelt zu sehen (11) (DM)

da beij hielt ich sehr streng auf tugend und Rechtschafenheit | und so wagt Es niemand mir durch das gegentheil zu nahe zu treten. (1) (DM)

Es zog mit mihr von Oberehn hinweck ein Man, so alda in den Weinreben 2 1/2 Jahr gearbeidtet hatt (41r) (G)

es wurde von da bericht es half aber nichts, armer du must leiden. (14) (J)

Ich thue jedoch gerne ein Vergnügen umsonst, es freut mich, wenn sich ein anderer freut. (8) (K)

Den ganzen Tag von früh bis abends musste ich streng arbeiten, so dass ich es für eine Ruh empfinde, wenn [ich] zum Gänsehüten geschickt werde. (16) (SCHG)

Und es dauert noch lange bis Du bei Deiner Lina bist. (1915) (LB)

wir wohnten zwar untter einem dache $\mid$ allein es währte doch ein zeitlang | Ehe wir uns sahen (1) (DM)

\section{Kapitel 2.3.3}

Sprecht eure Sprachen rein und klar, und erhaltet sie. Es ist wichtig. (24) (SB)

Die Frau hatte Erbarmen mit mir und sagte zu mir, so dass es niemand hörte sonst: «Geh ins Bett und schlaf, ich rufe Dich dann, bleib solang.> (15) (SCHG)

Könnte es nicht sein, daß also eine inflationäre Bewegung des Geldes eine natürliche oder eine notwendige Bewegung innerhalb des Wirtschaftssystems ist? (6) (SB)

Aber diese Bezeichnungen machen es ja eigentlich deutlich, wie man zu diesen Leuten steht (13) (SB)

Es war eben klar, daß die östlichen Medien zum Beispiel eine Angelegenheit wie den Einmarsch der Verbündeten in die Tschechoslowakei [...] eindeutig nach einer vorgegebenen Regelung formulierten $(8)(\mathrm{SB})$ 
Aber diese Bezeichnungen machen es ja eigentlich deutlich, wie man zu diesen Leuten steht (13) (SB)

Weiter wenn Du, mein Jakob, mir wieder Javakaffee von derselben Güte zu dem gleichen Preis wie den vorigen $1 / 2$ Zentner besorgen könntest, wärs recht. (17) (L)

Es ist auch uff den Sontag nach Jacobi ahngestellet worden im Land, alle Tag gegen Abend zu betten funf Vatterunser und funf Ave Maria und einen Glauben (31) (BL)

Die 1. Großmutter in Chrasic ist immer kränklich, sie weiß noch nichts vom Tode der 1. Tante Fleischner. Ja, ich kann es auch nicht glauben, u. doch ist es wahr, sie kann den schönen Frühling, nicht mehr athmen. (4) (KOR)

es were am allerbesten, wir blieben wie wir sein, ist doch nichts den hader und [1246] streit im dorffe gewesen (34) (NE)

Das ander aber, Lottern und Dachmachen, das verricht ich mit eygner Hand. Die Zeit wolt es nicht leiden, viel Leuth nemen, dan wie gehöret, wie es uns ergangen war, so konten wir nicht viel zum Besten haben (16) (BL)

es ist eine Eigenart dieser Krankheit, sich nach wiederholtem Schneiden wieder zu bilden. (4) (K)

es wäre zu wünschen, daß es ihm gut geht. (6) (K)

Ich weiß allerdings nicht mehr, ob ich denn, wie ichs euch mittheilte, gar nichts auch nur andeutungsweis beifügte, daß die Sach für jetzt noch nicht an die große Glocke gehört (17) (L)

Als ich nun heim kam, so schickt mir der Herr Magister gleich einen boten, und sagte recht zänckisch zu mir, seit ihr den schon wider kommen, Ja, was ist den draus worden, ich kans ihm selber nicht sagen was draus werden wil. (28) (NE)

Von da ab wird mir berichtet vom Schwabenland, ich stellte es mir natürlich so schön vor, dass mich niemand davon abhalten konnte nicht mitzugehen. Auch beim Zehrpfennigsammeln habe ich mitgeholfen. Es war erlaubt, dass die Kinder, die ins Schwabenland müssen, in den Nachbarsgemeinden Geld sammeln dürfen für die Reise, damit die Kinder nicht den ganzen langen Weg zu Fuss machen müssen. Auch da hab ich immer am meisten bekommen, weil ich das jüngste von den Schwabenkindern war. Heimlich hat es mich dann doch oft gereut, dass ich gesagt habe, ich wollte auch mit, hab manche Träne nachts im Bett vergossen und hatte zum voraus schon Heimweh. (3) (SCHG)

daß es der ewige Schlaf ist, den sie schläft, daß sie - kaum erwachen werde. Und so war es gekommen. Sie - ist um $6 \mathrm{Uhr}$ eingeschlafen, hat bis Mitternacht noch geathmet, u. ist dann verschieden. (24) (K)

\section{Kapitel 2.4.}

kein Brodt wahr auff dem Landt zu bekomen, dan es wahr auffgezertt von den Soldaten (54r) (G)

Mein Pferd war das Schießen nicht gewohnet und ging mit mir durch, wie ein Holländer. Da war kein Haltens. Weil im Trinken den Zügel fallen lassen, so hatte es seinen freien Lauf. (16) (MD) 
Erst machte das Ding Bedenklichkeiten; nachwerts bot es sich selber an. (9) (B)

da sagt mein mann | in seiner heimat stände ein schönes haus zu verkaufen. es stunde an der landstraße $\mid$ es gehörte dem fürst von witgenstein (7) (DM)

Ich suchte das Kind zu schützen, indem ich es in mein Zimmer mit dem Strickzeug setzte u. ihr nachher auch dort Stunde gab. (104) (SCH)

Mir ist es halt nicht gegönnt, Dir dies Ringl so meuchlings an die Hand zu stecken, wie Du's mir gethan, denk' aber indem Du es an Deinem Finger siehst, (68) (LB)

Ich halte es also für unwahrscheinlich, daß dieses ständig geübte Vokabular nicht auch in der Familie auftauchte. Das geht wahrscheinlich gar nicht anders. ... Und es tauchte auch auf. (17) (SB)

Aber ganz bewußt ... viele Dinge. ich weiß nicht, ob 'Traktor' deutsch ist ... 'Traktor' ist russisch. Ich weiß noch, daß damals, manchmal ein bißchen lustig, aber es ging dann plötzlich in den Sprachgebrauch über (18) (SB)

Das Gepolter im Haus währete ziemlich lange, bis endlich etwas zur Treppen herunterkam, über das Haus, im Hof zu plumpen anfing. Jch wußte, daß sonst kein Mensch im Hause war. Deswegen die Furcht und Angst sich mehrete. Aus dem Hof kam es rein in die Küche. (15) (MD)

«Was hast am Finger? Zeig, es wird bald reif, wärme fleissig auf, füttere die Gäns im Stall und bleib daheim! (14) (SCHG)

kamen auch bißweilen aus andern dörffer meine bekanden, und fragten beÿ mir umb diß und das, so sagt ichs ihnen nach aller einfald aus unsern Gothaischen Catechißmo (19) (NE)

Doch alles pfützennaß, daß’s im Augenblick, wie ein Harnisch, gefroren. (7) (MD)

Jedes sagte, was es gethan und gerathen, um mich wieder bey ihnen zu haben. (7) (B)

dieses alles württe mir eine neue mühe machen, wen ichs ein mal schreiben wolde. weil ichs aber dem Herrn professor Francke vor etligen Jahren schon neben andern dingen zu geschickt, sie werdens auch wol finden. (30) (NE)

Will also nur sagen, können wir nicht das, was Du für Patscherei ansahest, auch so ansehen, wie ichs eben ansah, oder müßen wirs unwedersprechl für Patscherei nehmen? (16) (L)

Ists nicht Wirklichkeit, sonder nur Schein, was mich zu reden trieb, so hats natürlich gar kein Gewicht (18f.) (L)

Im Jahr 1646 da zoge die schwedische Armee hir im Land herumb und verderbten es jämerlich (17) (BL)

Von da ab wird mir berichtet vom Schwabenland, ich stellte es mir natürlich so schön vor, dass mich niemand davon abhalten konnte nicht mitzugehen. (3) (SCHG)

So halfterte ich mich endlich von dem Wasserholen los, auf die Art: Jch thät, als stolperte ich mit den beiden vollen Wasserhosen und goß's also über den ganzen Saal und Haus, daß alles schwamm. (6) (MD) 
Sobald ich das Tier nicht richtig gerupft hab, so hatte es gejuckt und geschrien. An manchem Ort hats geblutet, das arme Tier. (13) (SCHG)

Dieses Ewig, ich kann es nicht ganz fassen, der menschliche Verstand ist zu schwach um etwas Unendliches zu fassen. (2) (K)

da sagt mein mann | in seiner heimat stände ein schönes haus zu verkaufen. es stunde an der landstraße | es gehörte dem fürst von witgenstein - es häten drei alte fräuleins bewohnt | wovon die eine noch lebte (7) (DM)

Wir in Stausenbach musten in geben 120 Reichstaller. Ach wie mancher vergoß die heisen Trenen daruber, biß wir es zusamen brachten. (8) (BL)

so waren wir einem manne $\mid$ der schon Viel geschalte mit uns gemacht hate - nur noch 13 Reichstaler schuldich - er hate uns eingeklagt | was ich aber nicht wuste - müller hatte die schrift verstekt - er ließ die 2te und 3te schrift komen. ohne das ich es wuste häte er es nun auch heimlich bezahlt $\mid$ so häte sein verheimligen doch einen grund gehabt (6f.) (DM)

Ich hatte das Mal in Kirchain elf Stuck Rindvieh und 17 Schwein und 4 Zigen. Wir musten gar vleisig wachen die Nacht, das es uns nicht gestollen ward von den Soltaden (14) (BL)

Bey ihme hatte ich Essen und Trincken, so ich es nuhr recht genießen kundte. (60r) (G)

Das Bier und Wasser in dem Städtchen wurde vom Viehe und so viel Menschen ganz ausgesoffen, daß nichts mehr zu bekommen war, und wir von weitem es holen mußten. (16) (MD)

Die Zeit ist wohl wieder recht gedrängt, aber wir werden's uns schon eintheilen, nicht wahr? (1787) (LB)

Zogen auch vor den Kirchain und wolten den einnehmen. Schossen auch uff 3 hundert Schüß mit Stucker wider die Mauer und in die Stadt. Schoßen auch die Mauer bey dem stumpen Thurn über ein Hauffen, aber sie liffens nicht an mit Sturm (13) (BL)

Der ließ die Kandtengießergesellen bey $\mathrm{i}[\mathrm{h}] \mathrm{me}$ nach des Handtwercks Prauch einziehen, gabe mihr Essen und Trincken, behielte mich uber Nacht, stellet mihr einen Schuncken auff. Dißer wahr voler Maden, dick wie ein Spindelspitz. Es geschmecket mihr aber woll (85v) (G)

Also das ist Alles vom Balle. Die 1. Mutter u 1. Gabi waren mit mir, eine seltene Ehre für den Ball. Jedenfalls gefiel es mir besser als voriges Jahr (2) (K)

Ich kan es gar nicht umbgehen und vergeßen, wie zuvor gedacht, der verwüsten und zerrisene Heuser und Bäuen in Stausenbach. (16) (BL)

zwei Gänserich haben mich geweckt. Ich habe mich herzlich gefreut und habs gestreichelt die zwei Tiere. (16) (SCHG)

Doch bekam er jetzt wieder in Aussig einen Posten, hoffentlich einen sicherern, als der letzte es war. (10) (K)

Der einte war, daß ich noch viel zu wenig Holz hatte, ungeachtet Mstr. Brunner mir gesagt, es sey genug (12) (B) 
der bekam abschlächlige antwort und wens auch ein Kavalir wahr er hate nun Rang und geld (2) $(\mathrm{DM})$

In dem ersae ich weitt vohr mihr etwaß fohr mihr ston, ungevohr ein halbe Meil Wœgx weitt. Als ich i[h]n erkandte, das es ein Man wahr, erschracke ich zim Teil (91v) (G)

Joseph sprach zu dem obersten schencken, und becker, auslegen der treume gehöret Gott zu, so hab ichs auch meinem Gott überlaßen der am besten wuste, was dieser traum beteuten wird, nur nach meiner Einfald habe ichs recht vor einen Göttlichen traum /gehalden/ (8f.) (NE)

da gieng's zuerst an ein lermendes, von Zeit zu Zeit mit Thränen vermengtes Freudengeschrey von Kleinen und Grossen, dann an ein Bewillkommen, Betasten und Begucken, Fragen und Antworten, daß es eine Tausendslust war. (7) (B)

Ich habe nicht Zeit zum Schreiben aber ich fühlte dieser Tage so das Bedürfniß es zu thun u. kann nicht dazu kommen. (1) (K)

wie mancher Punkt müßte von beiden Theilen besprochen werden u. wird's nur von dem einen. (108) (SCH)

müller nahm mich in der angst - auf seinen rücken und lief mit der bürde die höhe hinab - in das Dorf schameder, es war ein leinwebers haus was vorne im dorfe stand (9) (DM)

Endlich ver-Reißete der alte herr - und brachte einen neuen ofizianten mit namens Müller - dießer ach dießer mann war es $\mid$ der so viel einfluß auf mein nach übriges leben haben sollte (1) (DM)

Aber es waren auch 2 traurige Wochen, die auf den Ball folgten (1) (K)

Ich schrieb ihr, wie Du weißt, ich meine, es sind wohl 4 Wochen, u. hab keine Antwort mehr erhalten (12) (L)

\section{Kapitel 3.1}

eÿ höret doch liebe Mutter, es hat Ja ein Gottseliger Man diese bücher alle beide geschriben (3) (NE)

Da ward es mir gar kalt im Leibe. (23) (MD)

Der Großmutter geht es jetzt schon gut, doch hat sie diese letzten 2 Wochen viel gelitten (5) (K)

[...] als es nun rund in der stuben glimmete, so fingen hernach am ende rechte flammen /an zu brennen/, das es recht helle brande [...] (8) (NE)

es gibt schon leute in unserer gemeinde [...] (17f.) (NE)

ferner hat mich die sonderbare führung des lieben Gottes so weith gebracht, in dem ich nun erkenne, das er es recht gut mit mir gemeinet wie er zu davit gesaget im 32 Psalm. (5) (NE)

Es wurde auch, wenn von der Volkspolizei gesprochen wurde, nicht von den 'Bullen' gesprochen. (21) (SB) 
[...] den ich wuste selber nicht, wie mir war, wen ich auff der gaßen gieng [...] (36) (NE)

[...] endlich hörede etwas gantz dunckel bochen, ich lag aber stille, es bocht noch 2 oder 3 mal, so dachte ich, es were ein gespenst, endlich als es nicht wolte auff hören, und recht starck anschlug, so kroch ich heraus [...] (26) (NE)

[...] giengen sie in der hitten eine, assen und druncken, und namen silber, gold, und kleider, und gingen hin und verborgen es [...] (45f.) (NE)

[...] ich kans aber itzo nicht recht beschreiben wie dieses so wunderschön, mit meinen leibligen augen und ohren hörete ich dieses nicht, sondern nach dem inner geiste [...] (35) (NE)

\section{Kapitel 3.2}

ach meine liebe Mutter hat grose liebe und sorge vor mich, ach ich kan es dem lieben Gott und ihr nicht gnug dancken. (4) (NE)

Endlich trug sichs zu das ich nach Eisenach reisete zu meinem Bruder (2) (NE)

[...] da war recht in mir wirklich und empfindlich, wen es heist In Weinstock Jesu stehen wir, gepfropft, und gantz mit Gott vereinet [...] (12) (NE)

\section{Kapitel 3.3.2}

dan es donnert und hagelt, wen die Stuck abgingen (17) (BL)

Ist bald alles erloschen vom Funken, so gehts heim zur Mutter zum Küchleessen (2) (SCHG)

Es war doch erst März und noch ziemlich kalt (4) (SCHG)

Alles mögliche dachte ich mir, es war mir Angst und bang dabei. (7) (SCHG)

Ich fing an zu beten und dann weinte ich so, dass es mich schüttelte, bis ich gerufen wurde. (8) (SCHG)

Wer Zeit hatte, musste Obst auflesen; unter den Bäumen lag es dick von Obst (18) (SCHG)

Gar lustig ist es, wenn wir über die Zukunft reden, mit der Mamka spricht sich's so leicht Darüber... (1731) (LB)

Es wurde im Kränzchen nicht gelesen u. nur geschwatzt; ich hätte lieber zu Hause gesessen. (106) $(\mathrm{SCH})$

Die Sprache des Epos spricht mich an; es liegt der kindlich naive, kräftig deutsche Ton darin (101) $(\mathrm{SCH})$ 


\section{Kapitel 3.4}

so müßen den viele armen schafflein vor hunger und durst verschmachten und umbkommen, wegen solchern untreuen hirtten. so bleibt es doch beÿ den wortten des propheten, Ich wil einen hunger $\mathrm{p}$. $\mathrm{O} !(47)(\mathrm{NE})$

Zur 3. Wurzelziehen ist es heute nicht mehr gekommen. (636) (LB)

Morgen Abends erwarte ich Anna, Du siehst an Abwechslung fehlt's mir nicht... (787) (LB)

Jetzt ist's halt wieder aus mit der Freiheit... (1689) (LB)

Als die Zimmerleuth' fertig waren, giengs ans Mauern. (13) (B)

Diese Krankheit kam mir so ekelhaft vor, daß ich's sogar bey meinen Kindern nie recht ohne Grausen aushalten konnte. (22) (B)

Wie ist's mit Deiner Reise, ich weiß ja diesmal gar nicht wohin Du gehst. (513) (LB)

Mir ist es halt nicht gegönnt, Dir dies Ringl so meuchlings an die Hand zu stecken, wie Du's mir gethan, denk' aber indem Du es an Deinem Finger siehst, daß ich's im Geiste thue und daß ich Dich bitte es zu tragen zum Zeichen unserer Herzensgemeinschaft und daß endlos wie der Ring auch meine Liebe zu Dir ist. - Schreibe mir halt ob ich's mit der Weite getroffen habe, sonst müßte er gerichtet werden. (73) (LB)

Der hiesigen Großmutter geht es schlecht. (23) (K)

Naja, es gab einen offiziellen Sprachton, den sprachen alle Verantwortungsträger (3) (SB)

ich bitt Dich denn sieh, es handelt sich ja wahrhaftig nicht drum, daß ich als Richter über Dir sitzen will (16) (L)

Jch wußte nicht, mit wem ich's zu thun hatte. (13) (MD)

Nach diesen mich unangenehm berührenden Mittheilugen gingen wir zu Marie Schwarz zum Kaffee; Milia fand es recht behaglich dort (107) $(\mathrm{SCH})$

Bachmann hingegen hatte noch von seinem Handgeld übrig, gieng in die Schenke, und ließ sich's wohl schmecken; nur etwa zu vornehmen Häusern, Pfarrhöfen und Klöstern, kam er auch mit. (5) (B)

Ich fing an zu beten und dann weinte ich so, dass es mich schüttelte, bis ich gerufen wurde. (8) (SCHG)

Aber natürlich, den ganz negativen Anstrich an diesem Dialekt haben die Kommunisten gegeben, weil eben Sachsen dummerweise die meisten dieser Kommunisten gestellt hat, und die Kommunisten ja alle sächsisch sprachen, weil sie aus dieser Gegend hier kamen...vielfach zumindestens....Das hat natürlich dann „Kommunismus gleich sächsische Sprache“ eingebracht. Das ist nun einmal so... Es ist einfach so...daß die „rote“ kommunistische Arbeiterbewegung hier in Sachsen doch am allerausgeprägtesten war, und daß die roten, kommunistischen Funktionäre natürlich aus Sachsen kam, weil die Partei hier am stärksten war. (18) (SB) 
Es war also durchaus nicht so, daß sich da säuberlich getrennte Gruppen gegenüberstanden. Es war mehr so ... als ich dort in Z. war, das war ja eine verhältnismäßig späte Zeit (5) (SB)

Konnte sie aber nicht im Wolmeinen gegen Dich, allerdings ohne Deinen Auftrag, dann aber nur um so mehr im Wolmeinen mit Dir der andern bemerkl machen daß sie dich unmutig gemacht? Und ich glaub auch da, daß es so ist (15)

Vielleicht ist es auch die Trauer, die sie so schweigsam macht (22) (K)

Denn es ist hir in ähnlicher Weise wie bei den Bekenntnißschriften, daß man nicht das gegebene erforschen will, sondern selbst erst etwas aufrichten. (9) (L)

denn die jetzige traurige Zeit hat den fröhlichen Übermut von allen Menschenkindern abgestreift, es ist wie wenn über allen Menschen ein Trauerschleier niedergefallen wäre (22) (K)

er wüste nur nicht | wo sein schwager bliebe, oder wie es seij | das er nicht komme (8) (DM) 